\title{
The influence of CMAQ model resolution on predicted air quality and associated health impacts
}

By

\author{
Melanie Fillingham
}

A thesis submitted to the Faculty of Graduate and Postdoctoral Affairs in partial fulfillment of the requirements for the degree of

Masters of Applied Science

$\ln$

Environmental Engineering

Carleton University

Ottawa, Ontario

(C) 2019

Melanie Fillingham 


\section{Abstract}

Air quality models (AQM) paired with epidemiological data are often used to estimate health related burdens from pollution exposure. The accuracy of these predictions depends, among other factors, on the horizontal resolution of the model. This thesis aims to quantify the impact of horizontal grid resolution by simulating health impacts due to $\mathrm{O}_{3}, \mathrm{NO}_{2}$, and $\mathrm{PM}_{2.5}$ exposure using various model and input resolutions.

Adjoint sensitivity analysis was used to predict health impacts, producing results with respect to emissions sources. The results indicate that coarse modeled health impacts underestimate maxima in urban areas and overestimate in rural areas with proximity to urban cores. Additionally, coarse modelling does not sufficiently display spatial variance. Concentration exposure relationships applied to AQM predictions were also used to estimate health impacts. The resolution of individual inputs (meteorology, emissions, and population) were altered to examine which processes were most responsible for the differences due to resolution. The resolution of population had the largest impact on health impact results for all species. The resolution of meteorology and emissions impacted the species to different extents; $\mathrm{O}_{3}$ was more impacted by meteorology, while $\mathrm{NO}_{2}$ and $\mathrm{PM}_{2.5}$ were more influenced by emissions.

The influence of specific emission sources can be more adequately determined at fine resolution, benefiting air quality control. Aggregated estimates across the domain did 
not alter significantly between resolutions. It is suggested that justification based on the purpose of the analysis be considered prior to choosing a resolution. 


\section{Acknowledgements}

I want to acknowledge the numerous amount of people who have helped and contributed to my completion to this thesis. First off, thank you to my supervisor Dr. Amir Hakami. Your advice, comments, guidance and specifically your patience, is much appreciated. Thank you for allowing me to pursue this degree and work towards a common goal.

Thank you to all my colleagues, whom I now consider friends, in the research group, Burak Oztaner, Robyn Chatwin-Davis, Angele Généreux, Sina Voshtani, Rebab Mashayekhi, Marjan Soltanzadeh, Shunliu Zhao, and Amanda Pappin. You guys have been beyond helpful with your knowledge and emotional support.

This research would not have been possible without the contributions and collaborations with consultants at RWDI, specifically Wayne Boulton, Greg Conley, Jeff Lundgren, and Martin Gauthier. I am grateful for these contributions and thank you for always being pleasant and generous during interactions.

I extend my appreciation to my family and friends. Mom, dad, Jeff, your support and encouragement played a crucial role in my completion of this degree. To my friends, many of whom I consider family, your beliefs in me was an essential motivation to 
finishing my masters. Thank you for always being there for me. Without you all, I would never be where I am today.

Finally, to my team at Golder, thank you for allowing me to pursue achieving my career goals while also having the time to complete my masters. Cami Taylor, thank you for your belief in me. You are an amazing mentor and I will forever appreciate your guidance.

Last, but not least, I am grateful for the generous support from faculty and students in the Civil and Environmental Engineering department here at Carleton University. The professors I have had the honour to TA for, and to the students I have taught who have given me inspiration. 


\section{Contents}

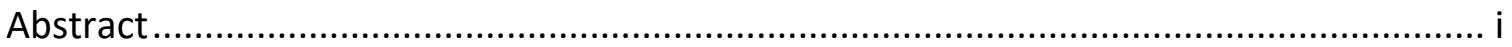

Acknowledgements..............................................................................................ii

List of Tables ..................................................................................................... vii

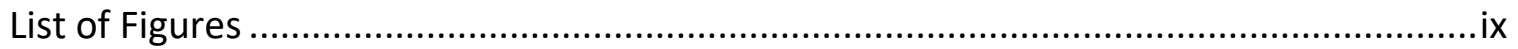

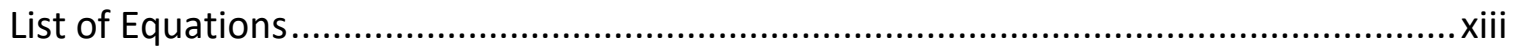

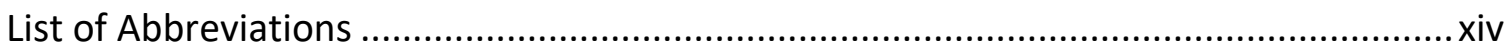

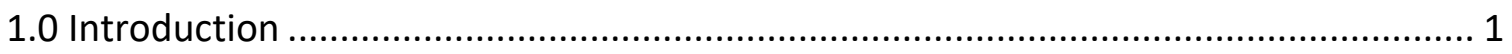

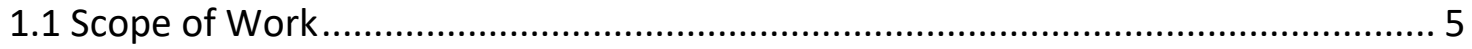

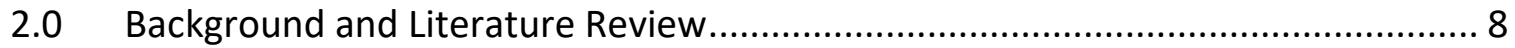

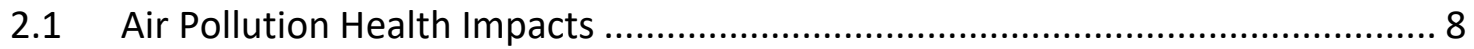

2.1.1 Ozone Exposure Epidemiological Studies ................................................... 10

2.1.2 Nitrogen Dioxide Exposure Epidemiological Studies ..................................... 11

2.1.3 Fine Particulate Matter Exposure Epidemiological Studies............................ 13

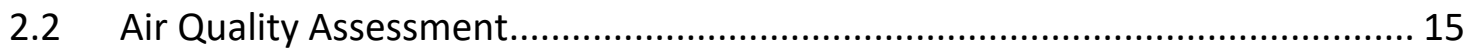

2.2.1 Air Quality Standards ......................................................................... 15

2.2.2 Air Quality Monitoring .......................................................................... 17

2.2.3 Air Quality Modelling ...................................................................... 18

2.3 Effects of Eulerian CTM Grid Resolutions ………........................................ 22

2.3.1 Effects of CTM Grid Resolution on Concentration Predictions .................. 23

2.3.2 Effects of CTM Grid Resolution on Health Impact Predictions ................... 34

2.3.3 Impacts from Meteorological Inputs Resolution ...................................... 38

2.3.4 Impacts from Emission Inputs Resolution ………....................................... 41

2.3.5 Trade-offs of Fine Resolution Modelling ……............................................ 43

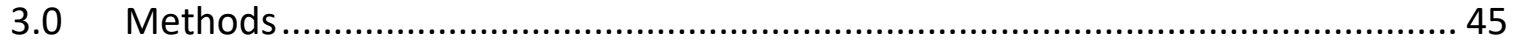

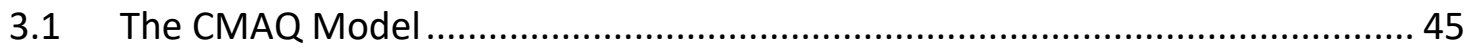

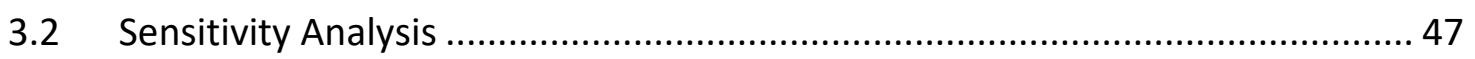

3.2.1 Forward Sensitivity Analysis: Brute Force Method................................... 49

3.2.2 Forward Sensitivity Analysis: Decoupled Direct Method .......................... 51

3.2.3 Adjoint (backward) Sensitivity Analysis ....................................................... 52

3.3 Estimation of Health Impacts ...................................................................... 54 
4.0 Impacts of grid resolution on adjoint-based marginal benefit estimates of air

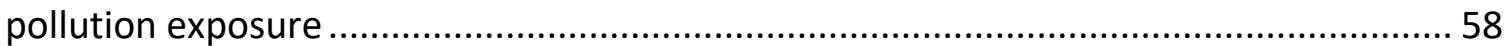

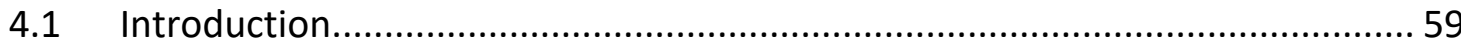

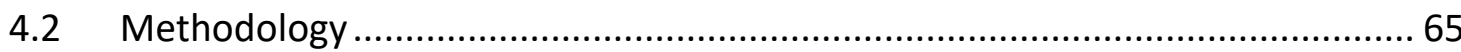

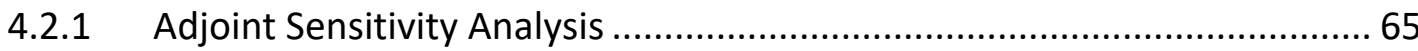

4.2.2 Health Valuation Parameters............................................................ 66

4.2.3 Case Study Model Parameters............................................................ 67

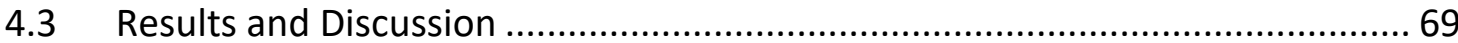

4.3.1 Concentrations: Progressively Refined Resolutions ................................ 69

4.3.2 Marginal Benefits: Progressively Refined Resolutions ............................ 78

4.3.3 Discussion of Limitations .................................................................. 89

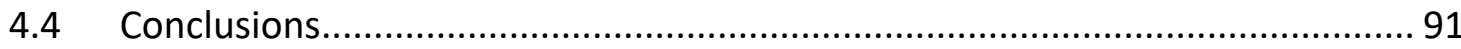

5.0 Assessing the Impact of CMAQ Input Resolution on Concentration and Associated

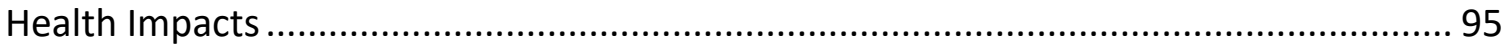

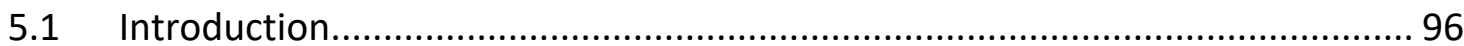

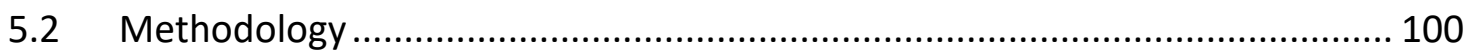

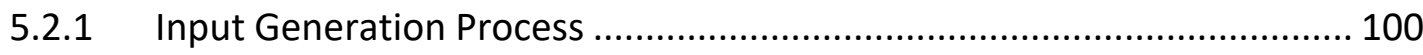

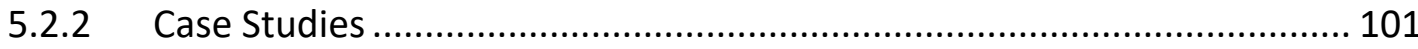

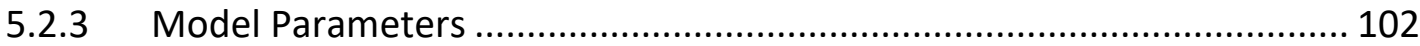

5.2.4 Exposure Health Predictions ................................................................. 104

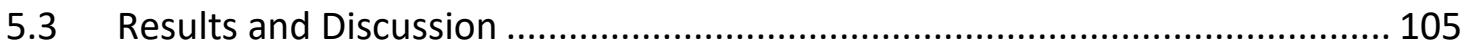

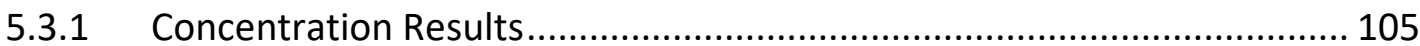

5.3.1.1 Ozone Concentration Results............................................................ 105

5.3.1.2 Nitrogen Dioxide Concentration Results......................................... 111

5.3.1.3 Fine Particulate Concentration Results............................................. 116

5.3.1.4 Resolution Impact Comparisons between Species ............................. 121

5.3.2 Exposure Health Impact Results ............................................................ 123

5.3.2.1 Ozone Related Health Impact Results............................................... 124

5.3.2.2 Nitrogen Dioxide Health Impact Results ........................................ 130

5.3.2.3 Fine Particulate Matter Health Impact Results.................................. 135

5.3.2.4 Resolution Health Impact Comparisons ............................................. 140

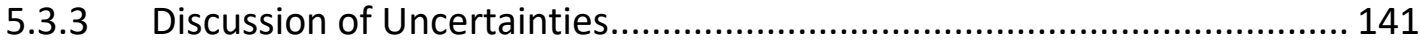

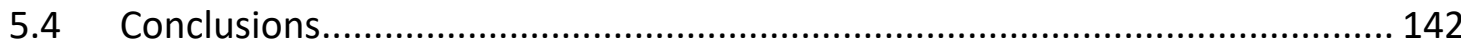




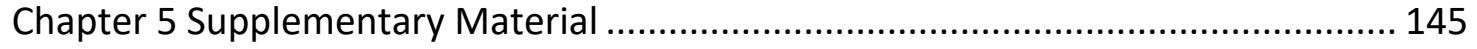

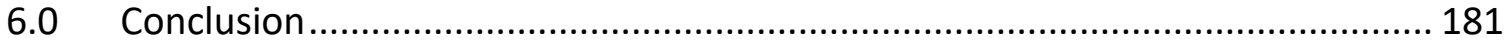

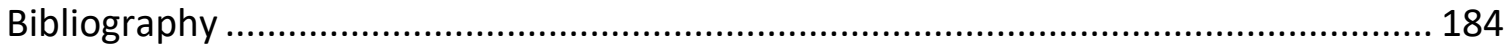

Appendix A: Ground-level Ozone Formation Chemistry ........................................... 201 


\section{List of Tables}

Table 2-1. Relevant jurisdictional air quality standards related to the pollutants assessed

within this thesis. 16

Table 4-1. Maximum modelled concentrations of $\mathrm{O}_{3}, \mathrm{NO}_{2}$ and $\mathrm{PM}_{2.5}$ at each resolution $(1,4,12$ and $36 \mathrm{~km})$ for the July and February results

Table 4-2. Maximum marginal benefit results from each resolution (1, 4, 12 and $36 \mathrm{~km})$.

Table 4-3 . Total domain-wide monthly damage results from each resolution $(36,12,4$ and $1 \mathrm{~km})$.

Table 5-1 Input resolution combinations assessed, where an italicized lower-case letter indicates a fine resolution input and a non-italicized upper-case letter indicates a coarse resolution input. 102

Table 5-2. RMSD (in ppb) for $\mathrm{DM}_{8 \mathrm{~A} \mathrm{O}} \mathrm{O}_{3}$ concentrating for each input resolution scenario.

Table 5-3. RMSD (in ppb) for 24-hour average $\mathrm{NO}_{2}$ concentrations for each input resolution scenario 116

Table 5-4. RMSD for 24-hour average $\mathrm{PM}_{2.5}$ concentrating for each input resolution scenario for all results (top) and with the extreme outlier removed (bottom)

Table 5-5. Domain-wide total annual health impacts due to exposure to $\mathrm{O}_{3}$ 129

Table 5-6. RMSD for the monetized health impacts due to $\mathrm{O}_{3}$ exposure for each input resolution scenario

Table 5-7. Domain-wide total annual health impacts due to exposure to $\mathrm{NO}_{2}$ 134 Table 5-8. RMSD for the monetized health impacts due to $\mathrm{NO}_{2}$ exposure for each input resolution scenario, displayed in logarithmic scale. 135

Table 5-9 Domain-wide total annual health impacts due to exposure to $\mathrm{PM}_{2.5}$ 139 Table 5-10. RMSD for the monetized health impacts due to $\mathrm{PM}_{2.5}$ exposure for each input resolution scenario, displayed in logarithmic scale 140 


\section{List of Figures}

Figure 2-1: Map of the Greater Toronto Area showing the locations of air quality

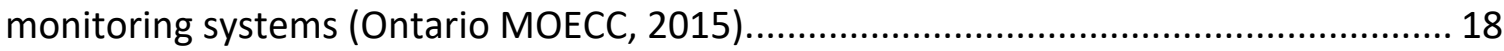

Figure 2-2. Inputs to an Eulerian CTM to estimate health impacts................................ 23

Figure 3-1. The CMAQ modelling system and required processors (adapted from Washington State University, 2018).

Figure 4-1. Nested domain configuration, resolutions are 36 (main domain), 12, 4 and 1 km (inner-most domain), respectively, focusing in on western Lake Ontario. 68 Figure 4-2. Concentration results for $36,12,4$, and $1 \mathrm{~km}$ (top to bottom) for $\mathrm{O}_{3}, \mathrm{NO}_{2}$, and $\mathrm{PM}_{2.5}$ (left to right), averaged to their respective standard averaging period and over the July 2 week period. 71 Figure 4-3. Concentration results for $36,12,4$, and $1 \mathrm{~km}$ (top to bottom) for $\mathrm{O}_{3}, \mathrm{NO}_{2}$, and $\mathrm{PM}_{2.5}$ (left to right), averaged to their respective standard averaging period and over the February 2 week periods. 72

Figure 4-4. Matrix of July first 2-weeks average $\mathrm{DM}_{8 \mathrm{~A} \mathrm{O}} \mathrm{O}_{3}$ concentrations aggregated to each separate coarse resolution. 76

Figure 4-5. Matrix of July first 2-weeks average 24-hour $\mathrm{NO}_{2}$ concentrations aggregated to each separate coarse resolution. 77

Figure 4-6. Matrix of July first 2-weeks average 24-hour $\mathrm{PM}_{2.5}$ concentrations aggregated to each separate coarse resolution. 78 Figure 4-7. July (left) and February (right) MB results at resolutions of 36, 12, 4 and $1 \mathrm{~km}$ (top to bottom). 81

Figure 4-8. July MB resolution comparisons using box and whisker plots at a) a predominantly urban location and at (b) a predominantly rural location..... 84

Figure 4-9. February MB resolution comparisons using box and whisker plots at a) a predominantly urban location and at (b) a predominantly rural location. 85 Figure 4-10. Monthly damage result for July (left) and February (right) for 36, 12, 4 and 1 $\mathrm{km}$ resolutions (from top to bottom).

Figure 5-1 Domains within the analysis, inner to outer domains for the original 1, 4 and $12 \mathrm{~km}$ model domains, respectively. 103

Figure 5-2. Daily Maximum 8-hour average $\mathrm{O}_{3}$ concentration results from various fine and coarse input resolution variation scenarios of 12 and $36 \mathrm{~km}, 4$ and $12 \mathrm{~km}$, and 1 and $4 \mathrm{~km}$ (top to bottom, respectively)...... 109

Figure 5-3. Scatter plots comparing the $\mathrm{DM} 8 \mathrm{~A} \mathrm{O}_{3}$ all fine resolution concentration results to concentrations generated with coarse emissions (left) and coarse meteorology (right), for resolution variation scenarios of 12 and $36 \mathrm{~km}, 4$ and $12 \mathrm{~km}$, and 1 and $4 \mathrm{~km}$ (top to bottom, respectively).

Figure 5-4. 24-hour average $\mathrm{NO}_{2}$ concentration results from various fine and coarse input resolution variation scenarios of 12 and $36 \mathrm{~km}, 4$ and $12 \mathrm{~km}$, and 1 and $4 \mathrm{~km}$ (top to bottom, respectively) 114

Figure 5-5. Scatter plots comparing the 24-hour average $\mathrm{NO}_{2}$ all fine resolution concentration results to concentrations generated with coarse emissions (left) and 
coarse meteorology (right), for resolution variation scenarios of 12 and $36 \mathrm{~km}, 4$ and 12 $\mathrm{km}$, and 1 and $4 \mathrm{~km}$ (top to bottom, respectively).

Figure 5-6. 24-hour average $\mathrm{PM}_{2.5}$ concentration results from various fine and coarse input resolution variation scenarios of 12 and $36 \mathrm{~km}, 4$ and $12 \mathrm{~km}$, and 1 and $4 \mathrm{~km}$ (top to bottom, respectively) 118

Figure 5-7. Scatter plots comparing the 24-hour average $\mathrm{PM}_{2.5}$ all fine resolution concentration results to concentrations generated with coarse emissions (left) and coarse meteorology (right), for resolution variation scenarios of 12 and $36 \mathrm{~km}, 4$ and 12 $\mathrm{km}$, and 1 and $4 \mathrm{~km}$ (top to bottom, respectively).

Figure 5-8 Monetized annual health impacts due to Chronic $\mathrm{O}_{3}$ exposure from various fine and coarse input resolution variation scenarios of 12 and $36 \mathrm{~km}, 4$ and $12 \mathrm{~km}$, and 1 and $4 \mathrm{~km}$ (top to bottom, respectively). 126 Figure 5-9. Scatter plots comparing the monetized annual health impacts due to chronic $\mathrm{O}_{3}$ exposure results generated at all fine resolution with coarse population, for resolution variation scenarios of 12 and $36 \mathrm{~km}, 4$ and $12 \mathrm{~km}$, and 1 and $4 \mathrm{~km}$ (top to bottom, respectively).

Figure 5-10. Monetized annual health impacts due to Chronic $\mathrm{NO}_{2}$ exposure from various fine and coarse input resolution variation scenarios of 12 and $36 \mathrm{~km}, 4$ and 12 $\mathrm{km}$, and 1 and $4 \mathrm{~km}$ (top to bottom, respectively).

Figure 5-11 Scatter plots comparing the monetized health impacts due to chronic $\mathrm{NO}_{2}$ exposure results generated at all fine resolution with coarse emissions (left) and population (right), for resolution variation scenarios of 12 and $36 \mathrm{~km}, 4$ and $12 \mathrm{~km}$, and 1 and $4 \mathrm{~km}$ (top to bottom, respectively).

Figure 5-12. Monetized annual health impacts due to Chronic $\mathrm{PM}_{2.5}$ exposure from various fine and coarse input resolution variation scenarios of 12 and $36 \mathrm{~km}, 4$ and 12 $\mathrm{km}$, and 1 and $4 \mathrm{~km}$ (top to bottom, respectively)

Figure 5-13 Scatter plots comparing the monetized health impacts due to chronic $\mathrm{PM}_{2.5}$ exposure results generated at all fine resolution with coarse emissions (left) and population (right), for resolution variation scenarios of 12 and $36 \mathrm{~km}, 4$ and $12 \mathrm{~km}$, and 1 and $4 \mathrm{~km}$ (top to bottom, respectively). 138

Figure 5-14. Daily Maximum 8-hour average $\mathrm{O}_{3}$ concentration results from various 12 $\mathrm{km}$ (fine) and $36 \mathrm{~km}$ (coarse) input resolution variation scenarios and the differences between the respective scenario and the all fine $12 \mathrm{~km}$ model run.

Figure 5-15. Scatter plots comparing the $\mathrm{DM} 8 \mathrm{~A} \mathrm{O}_{3}$ concentration results for combinations of fine $12 \mathrm{~km}$ resolution with coarse $36 \mathrm{~km}$ inputs: emissions (top), meteorology (middle) and both emissions and meteorology (bottom).

Figure 5-16 Daily Maximum 8-hour average $\mathrm{O}_{3}$ concentration results from various $4 \mathrm{~km}$ (fine) and $12 \mathrm{~km}$ (coarse) input resolution variation scenarios and the differences between the respective scenario and the all fine $4 \mathrm{~km}$ model run.... 147 Figure 5-17. Scatter plots comparing the $\mathrm{DM} \mathrm{AA} \mathrm{O}_{3}$ concentration results for combinations of fine $4 \mathrm{~km}$ resolution with coarse $12 \mathrm{~km}$ inputs: emissions (top), meteorology (middle) and both emissions and meteorology (bottom). 148 
Figure 5-18. Daily Maximum 8-hour average $\mathrm{O}_{3}$ concentration results from various $1 \mathrm{~km}$ (fine) and $4 \mathrm{~km}$ (coarse) input resolution variation scenarios and the differences between the respective scenario and the all fine $1 \mathrm{~km}$ model run. 149

Figure 5-19. Scatter plots comparing the $\mathrm{DM} 8 \mathrm{~A} \mathrm{O} \mathrm{O}_{3}$ concentration results for combinations of fine $1 \mathrm{~km}$ resolution with coarse $4 \mathrm{~km}$ inputs: emissions (top), meteorology (middle) and both emissions and meteorology (bottom). 150 Figure 5-20. 24-hour average $\mathrm{NO}_{2}$ concentration results from various $12 \mathrm{~km}$ (fine) and 36 $\mathrm{km}$ (coarse) input resolution variation scenarios and the differences between the respective scenario and the all fine $12 \mathrm{~km}$ model run.

Figure 5-21. Scatter plots comparing the 24-hour average $\mathrm{NO}_{2}$ concentration results for combinations of fine $12 \mathrm{~km}$ resolution with coarse $36 \mathrm{~km}$ inputs: emissions (top), meteorology (middle), and emissions and meteorology (bottom).

Figure 5-22. 24-hour average $\mathrm{NO}_{2}$ concentration results from various $4 \mathrm{~km}$ (fine) and 12 $\mathrm{km}$ (coarse) input resolution variation scenarios and the differences between the respective scenario and the all fine $4 \mathrm{~km}$ model run. 153

Figure 5-23. Scatter plots comparing the 24-hour average $\mathrm{NO}_{2}$ concentration results for combinations of fine $4 \mathrm{~km}$ resolution with coarse $12 \mathrm{~km}$ inputs: emissions (top), meteorology (middle) and population (bottom). 154

Figure 5-24. 24-hour average $\mathrm{NO}_{2}$ concentration results from various $1 \mathrm{~km}$ (fine) and 4 $\mathrm{km}$ (coarse) input resolution variation scenarios and the differences between the respective scenario and the all fine $1 \mathrm{~km}$ model run.

Figure 5-25. Scatter plots comparing the 24-hour average $\mathrm{NO}_{2}$ concentration results for combinations of fine $1 \mathrm{~km}$ resolution with coarse $4 \mathrm{~km}$ inputs: emissions (top), meteorology (middle) and population (bottom). 156

Figure 5-26. 24-hour average $\mathrm{PM}_{2.5}$ concentration results from various $12 \mathrm{~km}$ (fine) and $36 \mathrm{~km}$ (coarse) input resolution variation scenarios and the differences between the respective scenario and the all fine $12 \mathrm{~km}$ model run.

Figure 5-27. Scatter plots comparing the 24-hour average $\mathrm{PM}_{2.5}$ concentration results for combinations of fine $12 \mathrm{~km}$ resolution with coarse $36 \mathrm{~km}$ inputs: emissions (top), meteorology (middle) and population (bottom). 158 Figure 5-28. 24-hour average $\mathrm{PM}_{2.5}$ concentration results from various $4 \mathrm{~km}$ (fine) and $12 \mathrm{~km}$ (coarse) input resolution variation scenarios and the differences between the respective scenario and the all fine $4 \mathrm{~km}$ model run. 159 Figure 5-29. scatter plots comparing the 24-hour average $\mathrm{PM}_{2.5}$ concentration results for combinations of fine $4 \mathrm{~km}$ resolution with coarse $12 \mathrm{~km}$ inputs: emissions (top), meteorology (middle) and population (bottom). 160 Figure 5-30. 24-hour average $\mathrm{PM}_{2.5}$ concentration results from various $1 \mathrm{~km}$ (fine) and 4 $\mathrm{km}$ (coarse) input resolution variation scenarios and the differences between the respective scenario and the all fine $1 \mathrm{~km}$ model run. 161 Figure 5-31. Scatter plots comparing the 24-hour average $\mathrm{PM}_{2.5}$ concentration results for combinations of fine $1 \mathrm{~km}$ resolution with coarse $4 \mathrm{~km}$ inputs: emissions (top), meteorology (middle) and both meteorology and emissions (bottom). 162 
Figure 5-32 Monetized health impacts due to Chronic $\mathrm{O}_{3}$ exposure from various $12 \mathrm{~km}$ (fine) and $36 \mathrm{~km}$ (coarse) input resolution scenarios and the differences between the respective scenario and the all fine $12 \mathrm{~km}$ model run.

Figure 5-33. Scatter plots comparing the monetized health impacts due to chronic $\mathrm{O}_{3}$ exposure results for combinations of fine $12 \mathrm{~km}$ resolution with coarse $36 \mathrm{~km}$ inputs: emissions (top), meteorology (middle), and population (bottom). 164 Figure 5-34: Monetized health impacts due to chronic $\mathrm{O}_{3}$ exposure from various $4 \mathrm{~km}$ (fine) and $12 \mathrm{~km}$ (coarse) input resolution scenarios and the differences between the respective scenario and the all fine $4 \mathrm{~km}$ model run. 165 Figure 5-35. Scatter plots comparing the monetized health impacts due to chronic $\mathrm{O}_{3}$ exposure results for combinations of fine $4 \mathrm{~km}$ resolution with coarse $12 \mathrm{~km}$ inputs: emissions (top), meteorology (middle), and population (bottom). 166 Figure 5-36 Monetized annual health impacts due to chronic $\mathrm{O}_{3}$ exposure from various 1 $\mathrm{km}$ (fine) and $4 \mathrm{~km}$ (coarse) input resolution scenarios and the differences between the respective scenario and the all fine $1 \mathrm{~km}$ model run.

Figure 5-37. scatter plots comparing the monetized health impacts due to chronic $\mathrm{O}_{3}$ exposure results for combinations of fine $1 \mathrm{~km}$ resolution with coarse $4 \mathrm{~km}$ inputs: emissions (top), meteorology (middle), and population (bottom). 168

Figure 5-38. Monetized annual health impacts due to Chronic $\mathrm{NO}_{2}$ exposure from various $12 \mathrm{~km}$ (fine) and $36 \mathrm{~km}$ (coarse) input resolution scenarios and the differences between the respective scenario and the all fine $12 \mathrm{~km}$ model run....

Figure 5-39. Scatter plots comparing the monetized health impacts due to chronic $\mathrm{NO}_{2}$ exposure results for combinations of fine $12 \mathrm{~km}$ resolution with coarse $36 \mathrm{~km}$ inputs: emissions (top), meteorology (middle), and population (bottom). 170

Figure 5-40. Monetized annual health impacts due to Chronic $\mathrm{NO}_{2}$ exposure from various $4 \mathrm{~km}$ (fine) and $12 \mathrm{~km}$ (coarse) input resolution scenarios and the differences between the respective scenario and the all fine $4 \mathrm{~km}$ model run.

Figure 5-41. Scatter plots comparing the monetized health impacts due to chronic $\mathrm{NO}_{2}$ exposure results for combinations of fine $4 \mathrm{~km}$ resolution with coarse $12 \mathrm{~km}$ inputs: emissions (top), meteorology (middle), and population (bottom).

Figure 5-42. Monetized annual health impacts due to Chronic $\mathrm{NO}_{2}$ exposure from various $4 \mathrm{~km}$ (fine) and $12 \mathrm{~km}$ (coarse) input resolution scenarios and the differences between the respective scenario and the all fine $4 \mathrm{~km}$ model run.

Figure 5-43. scatter plots comparing the monetized health impacts due to chronic $\mathrm{NO}_{2}$ exposure results for combinations of fine $1 \mathrm{~km}$ resolution with coarse $4 \mathrm{~km}$ inputs: emissions (top), meteorology (middle), and population (bottom).

Figure 5-44. Monetized annual health impacts due to Chronic $\mathrm{PM}_{2.5}$ exposure from various $12 \mathrm{~km}$ (fine) and $36 \mathrm{~km}$ (coarse) input resolution scenarios and the differences between the respective scenario and the all fine $12 \mathrm{~km}$ model run. 175 Figure 5-45 scatter plots comparing the monetized health impacts due to chronic $\mathrm{PM}_{2.5}$ exposure results for combinations of fine $12 \mathrm{~km}$ resolution with coarse $36 \mathrm{~km}$ inputs: emissions (top), meteorology (middle), and population (bottom). 176 
Figure 5-46. Monetized annual health impacts due to Chronic $\mathrm{PM}_{2.5}$ exposure from various $4 \mathrm{~km}$ (fine) and $12 \mathrm{~km}$ (coarse) input resolution scenarios and the differences between the respective scenario and the all fine $4 \mathrm{~km}$ model run.

Figure 5-47. scatter plots comparing the monetized health impacts due to chronic $\mathrm{PM}_{2.5}$ exposure results for combinations of fine $4 \mathrm{~km}$ resolution with coarse $12 \mathrm{~km}$ inputs: emissions (top), meteorology (middle), and population (bottom).

Figure 5-48. Monetized annual health impacts due to Chronic $\mathrm{PM}_{2.5}$ exposure from various $1 \mathrm{~km}$ (fine) and $4 \mathrm{~km}$ (coarse) input resolution scenarios and the differences between the respective scenario and the all fine $1 \mathrm{~km}$ model run.

Figure 5-49. Scatter plots comparing the monetized health impacts due to chronic $\mathrm{PM}_{2.5}$ exposure results for combinations of fine $1 \mathrm{~km}$ resolution with coarse $4 \mathrm{~km}$ inputs: emissions (top), meteorology (middle), and population (bottom).

\section{List of Equations}

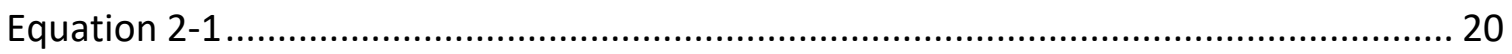

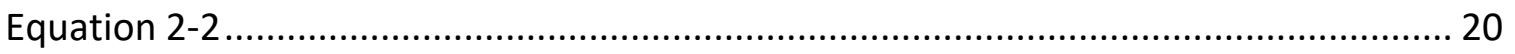

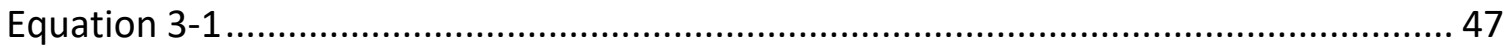

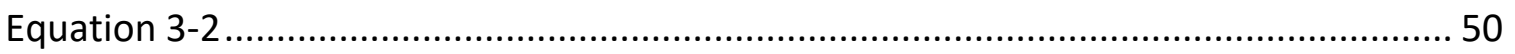

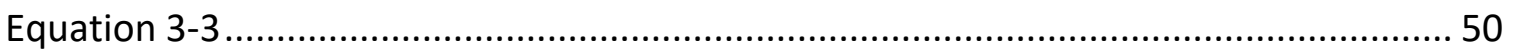

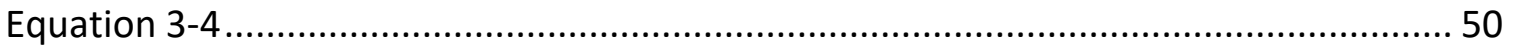

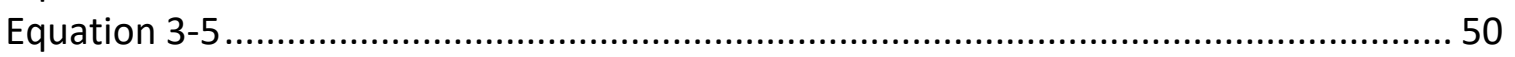

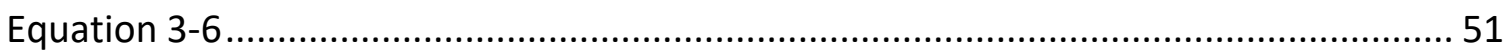

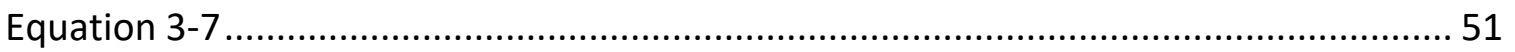

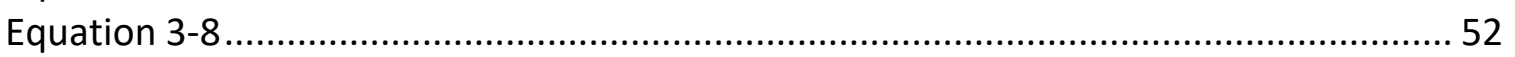

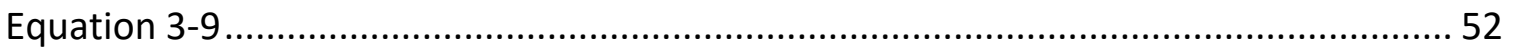

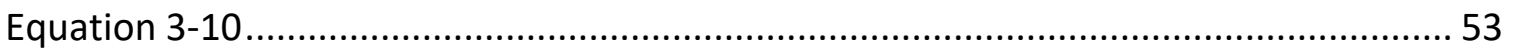

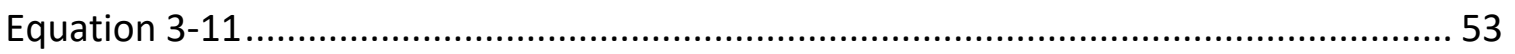

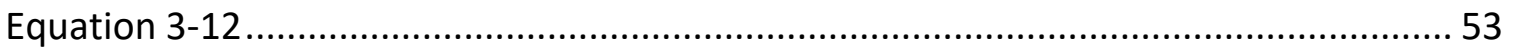

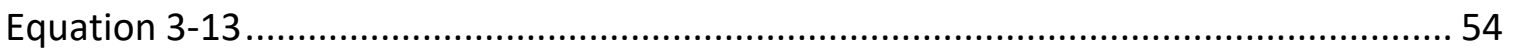

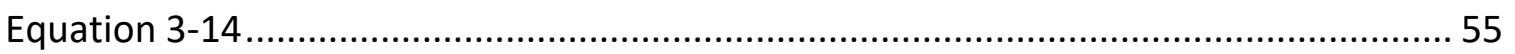

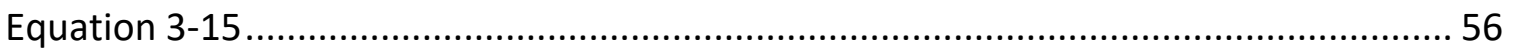

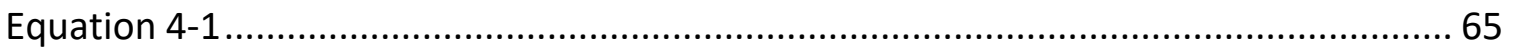

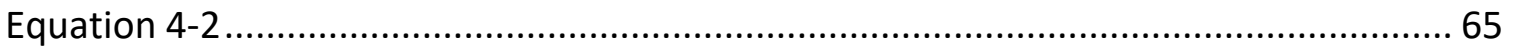




\section{List of Abbreviations}

ACS: American Cancer Society

ADE: Atmospheric Diffusion Equation

AQBAT: Air Quality Benefits Assessment Tool

AQM: Air Quality Model

AQMS: Air Quality Management System

BCON: Boundary Conditions Processor

BenMAP: Environmental Benefits Mapping and Analysis Program

BMR: Baseline Mortality Rate

BF: Brute-Force (sensitivity analysis method)

CAAQs: Canadian Ambient Air Quality Standards

CanCHEC: Canadian Census Health and Environment Cohort

CMAQ: Community Multiscale Air Quality

CPS-II: Cancer Prevention Study II (American Cancer Society)

CRF: Concentration-response factor

CTM: Chemical Transport Model

DDM: Decoupled Direct Method

DM8A: Daily Maximum 8-hour Average

ECCC: Environment and Climate Change Canada

EPA: Environmental Protection Agency

GTA: Greater Toronto Area

ICON: Initial Conditions Processor

ISA: Integrated Science Assessment

LHS: Left-Hand Side (equation)

MB: Marginal Benefit

MCIP: Meteorology Chemistry Interface Processor

NAPS: National Air Pollution Inventory

NEI: National Emissions Inventory

NOx: Nitrous Oxides (NO and NO2) 
PM: Particulate Matter

PM2.5: Fine Particulate Matter with aerodynamic diameter $<2.5 \mu \mathrm{m}$

RHS: Right-Hand Side (equation)

SMOKE: Sparse Matrix Operator Kernel Emissions model

VOC: Volatile Organic Compound

VSL: Value of Statistical Life

WHO: World Health Organization

WRF: Weather Research and Forecast 


\subsection{Introduction}

Air quality poses a significant challenge in our society due to its direct impact on human health and mortality, resulting in significant social and economic burdens. In 2016, an estimated 4.2 million deaths globally were a result of ambient air pollution exposure (WHO, 2018). Ground-level ozone $\left(\mathrm{O}_{3}\right)$ and nitrogen dioxide $\left(\mathrm{NO}_{2}\right)$ are pollutants that have suspected associations with adverse human health effects, while for fine particulate matter $\left(\mathrm{PM}_{2.5}\right.$, particulate matter with aerodynamic diameter $\left.<2.5 \mu \mathrm{m}\right)$ the association is established, especially related to cardiovascular and respiratory illnesses (Anenberg et al., 2010). Past research has suggested that both short-term and long-term negative health effects due to exposure to these pollutants (Burnett et al., 2004; Chen \& Kan, 2008I; Jerrett et al., 2009).

Estimation of the contribution of specific emission sources to human health impacts can help optimize pollutant control strategies for the utmost benefit. Air quality management relies on the control of emission sources. As a result, attributing air pollution health impacts to individual sources provides valuable information. The health consequences of these pollutants are dependent on the location and time of emission, causing spatiotemporal variability. The effects of air pollution on human health can be evaluated using benefit-cost analysis techniques that involve pairing chemical transport models (CTMs) with epidemiological data. The accuracy of these simulations are 
affected by a number of factors including the model's grid resolution and the ability to accurately represent spatial gradients.

Eulerian CTMs simulate the concentrations of air pollutants and their mechanics throughout the atmosphere in a 3-dimensional array of grid cells. The horizontal size (resolution) of these grid cells can alter the simulated concentrations. Coarse grid horizontal resolutions can limit the model's ability to accurately represent emissions, meteorology, and concentrations as implicit spatial averaging results in a single value in each grid cell that realistically would have spatial gradients. Coarse resolution grids also cannot adequately delineate population distributions, further impacting exposure related health predictions. Dependence on grid resolution is especially significant in urban/suburban areas where the spatial concentration gradients are strong (Thompson et al., 2014). Inaccuracies from CTM outputs can carry through and cause bias to the associated estimated health impacts. Additional to the impact of the resolution in which the CTM is run, the resolutions of the individual model inputs (meteorology, emissions and population) also carry through and impact the results. The extent in which resolution impacts concentration predictions is dependent on the species being observed (e.g. $\mathrm{O}_{3}, \mathrm{NO}_{2}, \mathrm{PM}_{2.5}$, etc.), the location of interest (e.g. rural vs. urban), and the scale of resolution (local, global, etc.). Generally, pollutants with short atmospheric lifetimes, and hence low transport ranges, are not well represented by coarse resolution models (U.S. EPA, 2007). 
Modelling at fine grid resolutions can reduce the inaccuracies that arise from grid averaging by better capturing spatial variabilities. Modelling at finer resolutions has higher computational costs associated which may not be feasible over large areas, such as global models. Each halving of a grid size (e.g. from $8 \mathrm{~km}$ to $4 \mathrm{~km}$ ) results in quadruple the number of grid cells required over the same domain. The storage size of fine resolution gridded data and results will be larger in magnitude than those of coarse resolution over the same domain. This leads to a trade-off between computational efficiency and storage, and model accuracy. One must consider whether the expected benefit of higher resolution can justify the higher costs. Modeling urban areas utilizing fine grid-resolutions and rural areas utilizing coarse grid resolutions can reduce inaccuracies while ensuring only problematic locations are analyzed at the high computational cost, optimizing both computational resources and model precision. One must also consider the availability of input data as some data, such as emissions or population, may not be readily available at very fine resolutions.

Sensitivity analysis can be used to examine the relationship between emission sources and receptor impacts. The health impact of individual source contributions over a defined domain can be estimated using a recent approach known as adjoint (backward) sensitivity analysis paired with epidemiological data. By pairing CTM sensitivity (change in concentration with respect to a change in emissions) with epidemiology data (in this research, change in health benefit due to a change in concentration exposure) and with economics data (in this research, monetized value of statistical life), one can determine 
what is known as marginal benefit (MB). Marginal benefit is defined as the change in monetized health impact due to a reduction in pollutant emissions; the monetized health benefit resulting from reducing emissions. This method of pairing adjoint (backward) sensitivity modeling of a CTM with epidemiological data has been used successfully to analyze $\mathrm{MB}$, however, these results were at a relatively coarse resolution of 36 km (Pappin et al., 2013a; Pappin et al., 2013b).

Previous research has attributed the effects of air pollution to emissions from specific sources (in terms of monetized health damage) and has found that health benefits associated with emission reduction were significant but highly dependent on the location of emission (Pappin \& Hakami, 2013a; Pappin \& Hakami, 2013b). High resolution (fine grid size) modelling also benefits sensitivity analyses as it allows for the impacts of individual emission sources on public health to be determined more accurately. The influence of individual emission sources is difficult to isolate with coarse resolution models as there may be multiple sources within the large grid cell.

Determining the contributions that individual emission sources have on health impacts provides information that is beneficial for formulating emission control policies. Sourcespecific benefit attributions provide decision makers with a direct measure of return of investments for policies that are aimed at improving air quality at large.

The research presented within this thesis analyzes impacts of grid resolution on both health impacts and health benefits. It is essential to be able to distinguish between the 
two terms. Health impacts refers to the impacts on human health due to air pollution exposure, calculated based on pollutant concentrations which in this research are predicted through CTMs. Health benefits refers to the human health benefits gained, or the reduction of negative health impacts, due to a reduction in emissions. Health benefits are calculated through CTM sensitivities, associated to the change in concentrations due to reducing emissions.

\subsection{Scope of Work}

The objective of the research presented within this thesis is to analyze the following questions:

1. What is the extent of impact of horizontal grid resolution on domain-wide predicted health impacts related to pollution exposure?

2. How does fine resolution modelling influence spatial and temporal patterns related to health benefits seen by emission reductions?

3. How does the resolution of specific model inputs (e.g. meteorology, emissions, population) impact health estimates?

4. Which CTM inputs provide a greater benefit to be of fine resolutions?

These above research questions are addressed through two experiments which are presented within this thesis as draft manuscripts. The remaining parts of this thesis are structured to support the research presented within these draft manuscripts. This thesis includes the following 6 chapters: 
Chapter 1: The first chapter provides a short introduction intended to bring context to the scope of the research and to clearly state the main objectives.

Chapter 2: This chapter provides a review of the literature that relates to the research presented within this thesis. Specifically, literature that concentrates on the topics of air quality and associated health impacts, and the impacts of model resolution.

Chapter 3: This chapter briefly outlines the methods used in the subsequent chapters, going further in-depth than the methods described within the later presented manuscripts.

Chapter 4: This chapter is presented as a draft manuscript which contains original research that analyzes the impacts of grid resolution on predicted emission reduction health benefits. This research involves simulating health impacts (particularly mortality) due to long-term $\mathrm{O}_{3}$ and $\mathrm{NO}_{2}$ (combined) exposure linked to $\mathrm{NOx}$ emission reductions (known as marginal benefit, MB) over progressively refined resolutions (36, 12,4 and $1 \mathrm{~km}$ ). This research is performed by pairing CMAQ-adjoint with epidemiology data. The MB predictions are compared between resolutions to draw conclusions on the impacts of resolution.

Chapter 5: This chapter is presented as a draft manuscript which contains original research analyzing the impacts of the resolution of specific chemical transport model (CTM) and exposure relationship input parameters on predicted health impacts (presented as mortality). The model inputs (meteorology, emissions and population) were altered between fine and coarse resolutions, one parameter at a time and for every combination. This was achieved through multiple CMAQ simulations with an 
exposure equation applied to the concentrations to estimate health impacts, over the same domain presented in chapter 4.

Chapter 6: The main conclusions are presented within the final chapter.

Recommendations for improvement and future work are given, and the limitations of this research are addressed.

The research presented in this thesis is intended to shed light on the impact of spatial resolution on the estimation of health impacts using CTMs. This research aims to aid modelers in choosing an optimal and appropriate grid resolution for the application at hand. The results from this study allow for air quality improvements, localized down to a $1 \mathrm{~km}$ resolution, to be monetized to aid decision-makers or urban-planners to focus emission reduction actions where benefits will be highest. 


\subsection{Background and Literature Review}

This chapter covers the background literature related to the focus of the thesis, which includes the following:

- Health impacts, specifically morality, associated with long-term chronic pollution exposure to $\mathrm{NO}_{2}, \mathrm{O}_{3}$ and $\mathrm{PM}_{2.5}$;

- Air quality models and their usage to estimate associated health impacts; and,

- Horizontal grid resolution impacts on CTMs and associated health predictions.

\subsection{Air Pollution Health Impacts}

Health impacts associated with poor air quality are a recognized global concern. Air quality standards exist primarily to protect public health. The World Health Organization (WHO) publishes ambient air quality guidelines which are intended to offer guidance globally for limits of key air pollutants related to human health risks (WHO, 2005). Countries are responsible for establishing their own standards, as example of which is Canada's implemented "Canadian Ambient Air Quality Standards." Furthermore, provinces or states can impose equal or more stringent standards, such as the Ontario air quality standards outlined in Ontario Regulation 419/05: Air Pollution - Local Air Quality. The health impacts related to $\mathrm{NO}_{2}, \mathrm{O}_{3}$, and fine particulate matter $\left(\mathrm{PM}_{2.5}\right)$ exposure is the focus of research within this thesis. This section goes through a brief review of the associated health impacts to these pollutants. 
Epidemiological studies relating to air pollution draw associations between pollution exposure and health impacts. These studies have demonstrated a variety of acute and chronic negative health effects due to both short and long-term exposure to $\mathrm{NO}_{2}, \mathrm{O}_{3}$, and $\mathrm{PM}_{2.5}$ (Burnett et al., 2004; Chen \& Kan, 2008; Jerrett et al., 2009). Adverse health consequences due to air quality include mortality, hospital admission, cardiovascular and respiratory disease, asthma attacks, and acute bronchitis. The Integrated Science Assessments (ISAs) released by the U.S. EPA provide the scientific evidence of pollutant related health and environmental impacts through extensive review and evaluation of relevant literature. The findings presented within the ISA for $\mathrm{PM}_{2.5}$ (U.S. EPA, 2009) indicates an association between both short and long-term exposure to these pollutants and health impacts, while the ISA for $\mathrm{O}_{3}$, (U.S. EPA, 2013) suggests a causal association. The findings from the 2008 ISA for $\mathrm{NO}_{2}$ were uncertain whether there was an association between health impacts and short or long-term exposure to the pollutant, however, the most recent $\mathrm{NO}_{2}$ ISA (U.S. EPA, 2016) indicates there is a relationship between short-term exposure and health impacts, and there is likely relationship between long-term exposure and health impacts.

The focus of review presented in this section is surrounding mortality associated with long-term pollution exposure, as this is the focus of the research presented within this thesis. The type of epidemiological studies that analyze long-term health risks due to pollution exposure are known as cohort studies. The results of these studies are often 
presented as the change in health outcome with respect to a change in pollutant concentration, known as an "effect estimate."

\subsubsection{Ozone Exposure Epidemiological Studies}

Numerous short-term exposure studies linking mortality and short-term $\mathrm{O}_{3}$ exposure have been performed such as those published by Bell et al. (2004), Burnette et al. (2004), and Katsouyanni et al. (2009), and the $\mathrm{O}_{3}$ ISA (2013) concludes that evidence supports a causal relationship. However, less literature is available linking mortality and long-term $\mathrm{O}_{3}$ exposure. The findings within the 2013 ISA for $\mathrm{O}_{3}$ indicate that the evidence suggests there is a causal relationship between long-term exposure and total mortality, respiratory mortality, and reproductive related mortality. This indication is due to the little available evidence on there being a causal relationship between longterm $\mathrm{O}_{3}$ exposure and an increase in human mortality.

The American Cancer Society (ACS) developed a cohort study known as the Cancer Prevention Study II (CPS-II) which demonstrated that elevated ozone concentrations increase the risk of respiratory mortality from chronic exposure (Jerrett et al., 2009). In this study, 448,850 subjects of at least 30 years of age were included from 96 U.S. urban areas between 1982 and 2000. The results found an effect estimate of $0.104 \% / p p b$ of daily maximum 1-hour $\mathrm{O}_{3}$ when looking at respiratory caused morality. A study performed by Krewski et al. (2009) used the ACS CPS-II cohort data but with different exposure data and found that all-cause mortality and $\mathrm{O}_{3}$ exposure (during the 
summertime) was found to have an effect estimate of $0.2 \% / \mathrm{ppb}$. Additionally, Turner et al. (2016) used the ACS CPS-II study results paired with estimated $\mathrm{O}_{3}$ concentrations at each participant's residence to examine the associations between long-term $\mathrm{O}_{3}$ exposure and mortality. This research found significant associations between long-term $\mathrm{O}_{3}$ exposure and all-cause mortality, circulatory mortality, and respiratory mortality.

Crouse et al. (2015) analyzed the Canadian Census Health and Environment Cohort (CanCHEC), studying the health impacts associated with long-term exposure to $\mathrm{O}_{3}, \mathrm{NO}_{2}$, and $\mathrm{PM}_{2.5}$. This cohort contained about 2.5 million Canadians that participated in the long-term 1991 Canadian census, who were over the age of 25. During a 16-year period, the annual pollutant concentrations $\left(\mathrm{O}_{3}, \mathrm{NO}_{2}\right.$, and $\left.\mathrm{PM}_{2.5}\right)$ that subjects were exposed to were estimated based off of the subjects' household postal code. Long-term $\mathrm{O}_{3}$ exposure was found to have an effect estimate of $0.188 \% / \mathrm{pbb}$ for non-accidental mortality. This study adjusted for $\mathrm{PM}_{2.5}$ and $\mathrm{NO}_{2}$ exposure. Cakmak, Hebbern, and Pinault (2017) also analyzed the CanCHEC data for associations between long-term $\mathrm{O}_{3}$ exposure and lung cancer-related mortality, finding there was not a significant relation.

\subsubsection{Nitrogen Dioxide Exposure Epidemiological Studies}

There is a lack of agreement as to whether long-term $\mathrm{NO}_{2}$ exposure is directly associated with mortality, or whether it is only an indicator of other pollutants such as PM. The evidence of the long-term effects of $\mathrm{NO}_{2}$ exposure on mortality was considered limited in the World Health Organization's (WHO) 2005 Air Quality Guidelines due to the 
few available studies. The findings within the $2016 \mathrm{NO}_{2}$ ISA indicate that available evidence suggests, but is not sufficient to conclude, that there is a causal relationship between long-term $\mathrm{NO}_{2}$ exposure and total mortality. This conclusion is based on the fact that the potential for confounding health impacts due to other traffic-related pollutants, or other factors, were uncertain at the time. Also in 2016, Turner et al. found that long-term exposure to $\mathrm{NO}_{2}$ was independently associated with mortality using the ACS CPS-II (previously discussed in Section 2.1.1). This research was not reviewed in the $2016 \mathrm{NO}_{2}$ ISA, however it further supports the evidence associating long-term exposure to $\mathrm{NO}_{2}$ and mortality.

Faustini et al. (2014) conducted a review of literature evaluating the link between $\mathrm{NO}_{2}$ exposure and mortality, applying a random-effects meta-analysis. They concluded that there is evidence of a relationship between long-term $\mathrm{NO}_{2}$ exposure and mortality. Their results found that the all-cause mortality effect was 1.04 with a $10 \mu \mathrm{g} / \mathrm{m}^{3}$ increase of annual $\mathrm{NO}_{2}$ concentration, cardiovascular mortality effect was 1.13 , and respiratory mortality was 1.03 . Using the conversion factor applied within this study $\left(1.88 \mu \mathrm{g} / \mathrm{m}^{3}\right.$ per ppb at $25^{\circ} \mathrm{C}$ and $1013 \mathrm{mb}$ ), these effects can be converted to effect estimates of $0.21 \% / \mathrm{ppb}, 0.69 \% / \mathrm{ppb}$, and $0.16 \% / \mathrm{ppb}$ for all-cause mortality, cardiovascular mortality and respiratory mortality, respectively. Crouse et al (2015) conducted an analysis on the relationship between long-term $\mathrm{NO}_{2}$ exposure and mortality in Canada using the CanCHEC cohort study (previously discussed in section 2.2.1). Crouse et al. concluded that there is a linkage between various cause 
of mortality and long-term $\mathrm{NO}_{2}$ exposure, with an effect estimate of $0.129 \% / p p b$ for non-accidental mortality.

\subsubsection{Fine Particulate Matter Exposure Epidemiological Studies}

Long-term exposure to $\mathrm{PM}_{2.5}$ has been shown to have a higher impact on public health than short-term $\mathrm{PM}_{2.5}$ exposure. There has been extensive research linking long-term exposure to $\mathrm{PM}_{2.5}$ and mortality (Pope et al., 2002; Laden et al., 2006; Miller et al., 2007; Eftim, Samet, Janes, et al., 2008; Zeger et al., 2008; Krewski et al., 2009; Crouse et al., 2012, 2016; Pinault, Tjepkema, Crouse, et al. 2016, 2017). A recent study incorporating 41 cohorts from 16 countries predicted globally 8.9 million deaths associated with $\mathrm{PM}_{2.5}$ annually (Burnett, Chen, Szyszkowicz, et al. 2018).

In 1993, Dockery, Pope, Xu, et al. published a cohort study on the effects of air pollution on mortality. This study, known as the "Harvard Six Cities Study," followed 8111 adults between 14-16 years within six U.S. cities, analyzing exposure based on pollutant concentrations measured at monitoring stations within the cities. The findings suggested that $\mathrm{PM}_{2.5}$ contributes to excess mortality in U.S. cities. This study can be seen as a pioneer in relating $\mathrm{PM}_{2.5}$ exposure and mortality, initiating the start to an abundance of studies on the topic.

The ACS CPS-Il cohort study (presented in Section 2.1.1) was used by Pope et al. (2002) to estimate chronic impacts of $\mathrm{PM}_{2.5}$ exposure. Pope et al. found an all-cause mortality 
effect estimate of $0.6 \%$ per $\mu \mathrm{g} / \mathrm{m}^{3}$ increase in annual average $\mathrm{PM}_{2.5}$. Krewski et al. (2009) also used the CPS-II cohort study and found an all-cause mortality effect estimate of $0.3-0.6 \%$ per $\mu \mathrm{g} / \mathrm{m}^{3}$ increase of annual average $\mathrm{PM}_{2.5}$. Eftim, et al. (2008) estimated and compared mortality from long-term $\mathrm{PM}_{2.5}$ exposure between the Harvard six cities cohort and the ACS CPS-II. The findings from this research were higher than those reported previously for both cohorts. They found that an increase in annual $\mathrm{PM}_{2.5}$ concentration by $10 \mu \mathrm{g} / \mathrm{m}^{3}$ was associated to an increase in all-cause mortality by $10.9 \%$ and $20.8 \%$ for the CPS-II study and the Six Cities study, respectively.

In Canada, Crouse et al. (2012) studied 2.7 million subjects (participants in the 1991 Canadian census) from 1991-2001 to establish a linkage between long-term $\mathrm{PM}_{2.5}$ exposure and mortality. Results from this study indicated an effect estimate for nonaccidental cause mortality of $1.4 \%$ per $\mu \mathrm{g} / \mathrm{m}^{3}$ increase of annual average $\mathrm{PM}_{2.5}$. This cohort used in Crouse et al. (2012) was later termed the CanCHEC study. Crouse et al. (2015) subsequently studied the CanCHEC cohort study (previously discussed in Section 2.2.1) to analyze the relationship between long-term $\mathrm{PM}_{2.5}$ exposure and mortality. In this study, the effect estimate of long-term $\mathrm{PM}_{2.5}$ exposure to non-accidental mortality was found to be $0.22 \%$ per $\mu \mathrm{g} / \mathrm{m}^{3}$ increase of annual average $\mathrm{PM}_{2.5}$. Pinault et al. (2017) used the 2001 CanCHEC cohort to study the associations between $\mathrm{PM}_{2.5}$ and mortality. This cohort study followed 2.4 million people between $25-90$ years of age over 10 years. In this research, they found an effects estimate of $1.6 \%$ per $\mu \mathrm{g} / \mathrm{m}^{3}$. Another recent study 
using the CanCHEC cohort found a significant risk on lung-cancer related mortality due to long-term $\mathrm{PM}_{2.5}$ exposure in Canada (Cakmak, et al. 2018).

Research has, and continues to, demonstrate the linkage between long-term exposure to $\mathrm{PM}_{2.5}$ and mortality. The findings within the 2009 ISA for $\mathrm{PM}_{2.5}$ conclude that there is evidence of a causal relationship between long-term exposure and total mortality, and cardiovascular effects (including mortality).

\subsection{Air Quality Assessment}

The assessment of air quality often involves the comparison of pollutant concentrations to air quality standards. The purpose of this section is to introduce air quality standards and to demonstrate the different methods that can be used to assess compliance to these standards. Since the research performed within this thesis used a chemical transport model (CTM), the explanation of CTMs will be more elaborate than other methods. The understanding of CTMs will aid in the understanding of the impact resolution has on these models.

\subsubsection{Air Quality Standards}

Air quality standards have been established to ensure concentrations of air pollutants with adverse human health impacts are and remain within safe limits that do not threaten human health. There are multiple tiers of air quality standards, starting with broad global guidelines and going all the way down to municipal level standards in some jurisdictions. The World Health Organization (WHO) publishes ambient air quality 
guidelines intended to offer global guidance for limits of key air contaminants known to have associated human health risks (WHO, 2005). Countries are responsible for establishing their own standards. Canada has implemented the "Canadian Ambient Air Quality Standards" (CAAQS), set out by the Canadian Council of Ministers of the Environment (ECCC, 2013a). The U.S. EPA has similar national standards, known as the "National Ambient Air Quality Standards" (NAAQS). Furthermore, provinces or states can additionally impose equal or more stringent standards, such as the Ontario air quality standards outlined in Ontario Regulation 419/05: Air Pollution - Local Air Quality.

Table 2-1 summarizes the relevant jurisdictional standards of pollutants presented within this research. The averaging period in which pollutant standards are set are based off epidemiological and toxicological studies which assess the impacts related to the time of exposure. Ozone's standard is based off the daily maximum rolling 8-hour average known as the daily maximum 8-hour average "DM8A," $\mathrm{NO}_{2}$ is based off the maximum 1-hour, and annual average, and the $\mathrm{PM}_{2.5}$ standard is based off 24-hour average, and annual average.

Table 2-1. Relevant jurisdictional air quality standards related to the pollutants assessed within this thesis.

\begin{tabular}{cccccc} 
& $\mathbf{O}_{\mathbf{3}}(\mathbf{p p b})$ & \multicolumn{2}{c}{$\mathbf{N O}_{\mathbf{2}}(\mathbf{p p b})$} & \multicolumn{2}{c}{$\mathbf{P M}_{\mathbf{2 . 5}}\left(\boldsymbol{\mu g} / \mathbf{m}^{\mathbf{3}}\right)$} \\
DM8A & $\mathbf{1 - h r}$ & Annual & 24-hour & Annual \\
\hline WHO, 2005 & 100 & 200 & 40 & 25 & 10 \\
CAAQS, 2015 & 63 & $60^{*}$ & $17^{*}$ & 28 & 10 \\
NAAQS, 2018 & 70 & 100 & 53 & 35 & 12 \\
\hline *CAAQS for $\mathrm{NO}_{2}$ are from 2020 proposed standards, $\mathrm{NO}_{2}$ standards for 2015 are not established
\end{tabular}




\subsubsection{Air Quality Monitoring}

Air quality monitoring stations are deployed to assess pollution concentrations in ambient air. In Canada, Environment and Climate Change Canada (ECCC) and provincial Ministers of the Environment work together to form the network of air quality monitoring stations across the nation. This is achieved through the National Air Pollution Surveillance (NAPS) program, established in 1969. NAPS currently exists of 286 monitoring sites which measure concentrations of criteria air pollutants such as $\mathrm{NO}_{2}, \mathrm{O}_{3}$ and PM (including $\mathrm{PM}_{2.5}$ ). The previous year's measured data can be publicly downloaded through ECCC's website. Within Ontario, there are 39 ambient air monitoring stations. The monitoring stations within the area of analysis presented within this thesis, Western Lake Ontario/the Greater Toronto area, are displayed in Figure 2-1. 


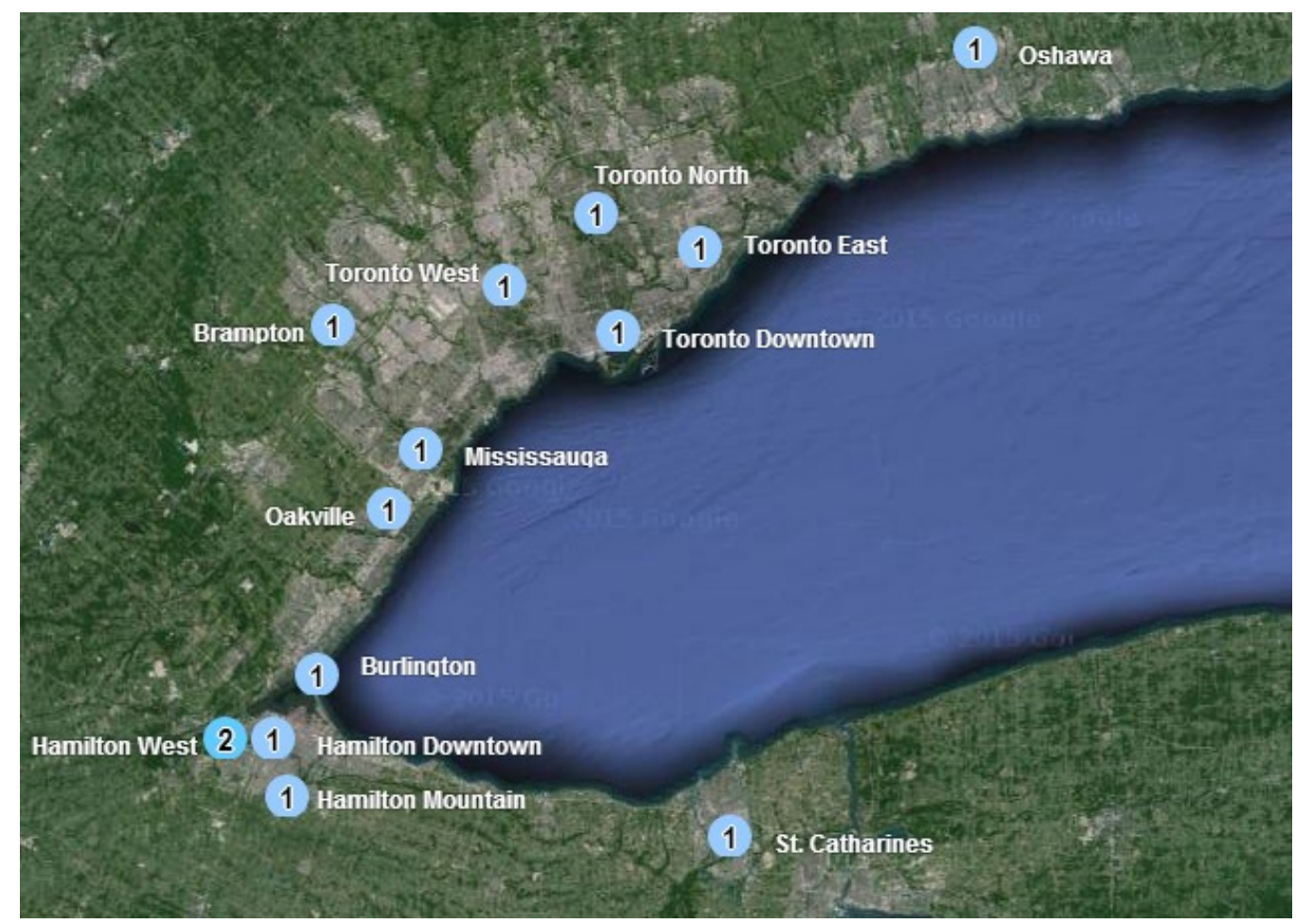

Figure 2-1: Map of the Greater Toronto Area showing the locations of air quality monitoring systems (Ontario MOECC, 2015).

\subsubsection{Air Quality Modelling}

Given the nature of the atmosphere, in-situ atmospheric experimental tests would be costly, time-consuming, and possibly pose a risk to human and environmental welfare, and therefore the field of atmospheric science and engineering is often reliant on modelling. Atmospheric modelling has many applications, such as pollution forecasting, policy development and assessment, consulting, and research.

Atmospheric air quality models (AQM), such as CTMs, are used to simulate how pollutants behave within the ambient atmosphere. These models range in application and complexity. One benefit of modelling is that there can be continuous spatial 
representation over the domain, as opposed to monitoring in which pollutant concentrations are only known at discrete monitor locations. The three more common model types are Gaussian, Lagrangian, and Eulerian models.

The most commonly used type of model for compliance assessment is the Gaussian dispersion model. Gaussian models assume that the dispersion of air pollutants follow a “Gaussian" or normal distribution. These models do not consider complex chemistry within the atmosphere. One commonly used Guassian model is AERMOD, a steady-state dispersion model which is designed for short-range dispersion (U.S. EPA, 2004). AERMOD is an approved U.S. EPA regulatory model, which is also accepted for compliance modelling within Ontario (O. Reg. 419/05). The frame of reference of this model is stationary, as the observer watches the plume move from an outside perspective.

Lagrangian (puff) models follow parcels of pollution throughout the atmosphere, transported by the prescribed wind field. These models are known for using a "moving frame of reference," as the observer moves along with the plume, as a first-person perspective (Jacob, 1999). One commonly used Lagranian model is "CALPUFF."

The most relevant model type to this research is the Eulerian (grid) chemical transport model. Eulerian models use defined 3-dimensional Cartesian grids as a frame of 
reference, as the observer watches the plume from a third person perspective (Jacob, 1999). Eulerian CTMs will be discussed in depth in the following section 2.2.3.1.

\subsubsection{Eulerian Atmospheric Chemical Transport Models}

Chemical transport models (CTMs) are Eulerian AQMs that include the chemical interactions of the atmosphere. They are more complex than simple dispersion models which only consider physical transportation within the atmosphere, excluding chemical formation or depletion pollutants may experience. Discretization of the continuous atmosphere in CTMs involves capturing representation by a finite number of grid cells.

The foundation of atmospheric CTMs is the atmospheric diffusion equation (ADE, Equation 2.1 and 2.2) (Seinfeld \& Pandis, 2006), which is integrated within each grid cell and at each time step.

$$
\begin{gathered}
\frac{\partial c_{i}}{\partial t}=\text { advection }+ \text { diffusion }+ \text { chemistry }+ \text { emissions }+ \text { deposition } \\
\frac{\partial C_{i}}{\partial t}=-u \cdot \nabla C_{i}+\frac{1}{\rho} \nabla \cdot\left(\rho \mathrm{K} \nabla \mathrm{C}_{\mathrm{i}}\right)+R_{i}+E_{i}+D_{i}
\end{gathered}
$$

Where: $C_{i}$ is the concentration of species $i$

$u$ is the 3-dimensional wind field

$\rho$ is the density of air

$\mathrm{K}$ is the turbulent diffusivity tensor

$\mathrm{R}_{\mathrm{i}}$ are the chemical reactions of species $i$

$\mathrm{E}_{\mathrm{i}}$ is the emission rate of species $i$

$D_{i}$ is the deposition velocity of species $i$ 
The left-hand side (LHS) of the equation, $\frac{\partial C_{i}}{\partial t}$, represents the change in concentration (of a defined species, i) over time within the grid cell, the accumulation or loss of concentration. The first two terms on the right-hand side (RHS) of the equation represent transportation, through advection $\left(-u \cdot \nabla C_{i}\right)$ and turbulent diffusion $\left(\frac{1}{\rho} \nabla \cdot\right.$ $\left.\left(\rho K \nabla C_{i}\right)\right) . R_{i}$ includes chemical/thermochemical transformation reactions that result in both production and loss of concentrations; there may be numerous chemical reactions represented by this term (e.g. for $\mathrm{O}_{3}$, there are several chemical reactions). $D_{i}$ is loss of concentration through deposition.

The turbulent diffusion within CMAQ contains both vertical and horizontal components. Simple first-order eddy diffusion is used for the horizontal diffusion, which is a function of a grid resolution dependent constant and the horizontal wind field. Horizontal diffusivity for a higher-resolution simulation is larger while numerical diffusion from advection is smaller (CMAS, 2012). The diffusion coefficient, which is dependent on grid resolution, is generated through the meteorological model and is an input to CMAQ.

Eulerian CTMs solve the ADE through the technique of operator splitting. The partial differential equations (PDE) that make up Equation 2.2 are solved by separating the PDEs into more simple ordinary differential equations (ODE) for each species and for only one or two processes. For example, advection is solved independently, for one species and in a single direction, as an ODE. This is applied for all PDEs in the ADE. The ODE solutions are then all combined as the solution of the ADE equation (Byun, 1999). 
For the intent of this research, it is important to keep in mind that the ADE is solved within each 3-D grid covering the modelling domain (and across grids for transport), resulting in one concentration value per grid cell, whether that grid cell is $36 \mathrm{~km}$ by 36 $\mathrm{km}$ or whether that grid cell is $1 \mathrm{~km}$ by $1 \mathrm{~km}$.

\subsection{Effects of Eulerian CTM Grid Resolutions}

Eulerian CTMs simulate the concentrations of air pollutants and their mechanisms throughout the atmosphere in a 3-d array of grids. When these CTMs are paired with epidemiological and population data, associated health impacts can be predicted. The resolution of the model grids can influence the accuracy of both the concentration and health impact predictions, as averaging occurs within each grid cell. Grid resolution impacts chemistry, transportation, and deposition processes. Coarse grid resolutions are limited in their ability to accurately represent spatial variability of parameters such as emissions, meteorology, concentrations, and population distributions.

Additional to the impact of the resolution at which the CTM is run, the resolution of the model inputs also impacts the results. The model inputs to a CTM include emissions and meteorology, along with initial and boundary conditions. When determining health impacts, population is also a required input. The following, Figure 2-2, visually displays the inputs required to the CTM CMAQ, and where the resolution impacts are seen. This 
displays a CTM in which the meteorology was run offline, or separate to the CTM. This research is focused on modelling with meteorology run offline.

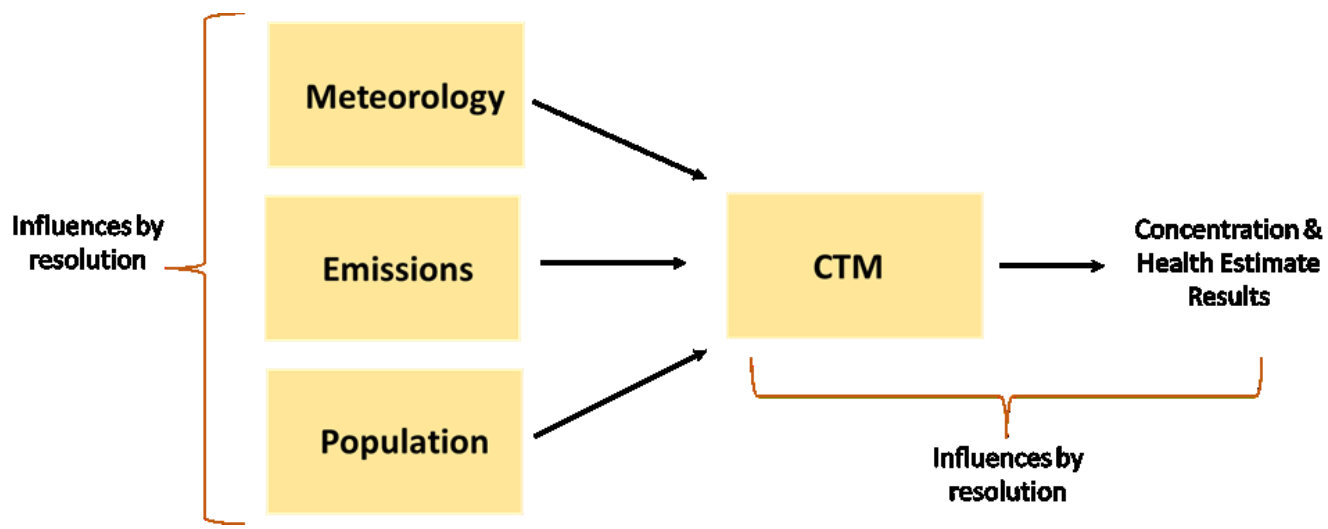

Figure 2-2. Inputs to an Eulerian CTM to estimate health impacts

This section covers a literature review of the impacts of grid resolution on CTM concentration predictions and associate health impacts related to $\mathrm{O}_{3}, \mathrm{NO}_{2}$ and $\mathrm{PM}_{2.5}$. It further reviews literature regarding the impacts of the resolution of CTM inputs (emissions, meteorology, and population) in regards to both concentration and health impact estimates.

\subsubsection{Effects of CTM Grid Resolution on Concentration Predictions}

Numerous studies have analyzed the impact of horizontal grid resolution on CTM results. The extent of influence of resolution depends on the species being investigated (e.g. $\mathrm{O}_{3}, \mathrm{NO}_{2}, \mathrm{PM}_{2.5}$, etc.), the domain of interest (e.g. rural vs. urban), and the scale of resolution (local, global, etc.). Generally, pollutants with short atmospheric lifetimes, and hence low transport ranges, are not well represented by coarse resolution models (U.S. EPA, 2007). Past research has demonstrated the response to grid resolution be 
strongest for $\mathrm{NO}_{2}$, followed by PM, and lastly for $\mathrm{O}_{3}$ (Queen \& Zhang, 2008; Schaap et al., 2015). The impacts of resolution on concentration predictions of the pollutants of focus within this thesis $\left(\mathrm{O}_{3}, \mathrm{NO}_{2}\right.$ and $\left.\mathrm{PM}_{2.5}\right)$ will be discussed within this section. There is an abundant amount of research indicating that $\mathrm{O}_{3}$ concentration predictions from CTMs are influenced by grid resolution (Jang et al., 1995a; Jang, Jeffries \& Tonnesen, 1995; Tang, 2002; Esler et al., 2004; Arunachalam et al., 2006; Cohan et al., 2006; Wild \& Prather, 2006; Queen \& Zhang, 2008; Valari \& Menut, 2008; Shrestha el at., 2009; Tie et al., 2010; Hodnebrog et al., 2011; Lauwaet et al., 2013; Pan et al., 2017). The U.S. EPA recommends modelling $\mathrm{O}_{3}$ within urban areas at a grid resolution of $4 \mathrm{~km}$ and advises against using a grid cell larger than $12 \mathrm{~km}$, often requiring resolutions as fine as $2 \mathrm{~km}$ to reproduce $\mathrm{O}_{3}$ chemistry (U.S. EPA, 2007).

Ozone is a relatively long-lived species with an average atmospheric lifetime in the northern hemisphere of 23 days (Seinfeld and Pandis, 2006). This causes its distribution to be more homogenous compared to short-lived pollutants, as it has time to travel and mix. It has been suggested that grid resolution does not influence long-lived species as significantly as those that are short-lived (US, EPA. 2007; Punger \& West, 2013). It has also been suggested that secondary pollutants, such as $\mathrm{O}_{3}$, are more sensitive to grid resolution as the uncertainty related to the resolution of meteorology, emissions, chemistry and transport all accumulates to the final $\mathrm{O}_{3}$ concentration (Hanna et al., 1998, 2001). The non-linear $\mathrm{O}_{3}$ chemistry with precursor species (NOx and VOCs) resulting in both formation and losses causes $\mathrm{O}_{3}$ to be particularly susceptible to impacts 
of grid resolution. The trend between atmospheric lifetime and grid resolution impacts may not be true for reactive secondary species with non-linear chemistry, such as $\mathrm{O}_{3}$. Refer to Appendix A for a detailed description on the non-linear $\mathrm{O}_{3}$ formation chemistry.

Past studies have demonstrated that relative to fine resolution CTM results, coarse resolution CTMs often underestimate $\mathrm{O}_{3}$ concentrations in urban areas, while in contrast overestimate $\mathrm{O}_{3}$ concentrations in nearby rural areas (Jang et al., 1995a; Liang \& Jacobson, 2000). This is a result of the artificial dilution of $\mathrm{O}_{3}$ precursors that occurs at coarse resolutions, often causing maxima to be underpredicted and minima to be overpredicted. Positive biases have been seen to occur as resolution becomes coarser, as resolution influences both chemical rates and transport processes (Kumar et al., 1994; Jang et al., 1995). Throughout this section, a summary of selected studies on the topic of Eulerian CTMs grid resolution and $\mathrm{O}_{3}$ concentration will be examined in detail, beginning with relatively coarse grid comparisons (with "fine" as $20 \mathrm{~km}$ ), and examining further into smaller grid comparisons (down to $<1 \mathrm{~km}$ resolution).

Jang et al. (1995) used the Eulerian grid model HR-RADM to assess $\mathrm{O}_{3}$ sensitivity to grid resolution, simulating $\mathrm{O}_{3}$ production at 20,40 , and $80 \mathrm{~km}$ horizontal resolution. In this research, the examination of the impact of grid resolution on $\mathrm{O}_{3}$ precursors (VOCs and NOx), and on individual meteorology and chemical processes was also assessed. It was found that $80-\mathrm{km}$ resolution could not resolve NOx and VOC emissions, causing underpredicted $\mathrm{O}_{3}$ maxima downwind of emission sources and overpredicted $\mathrm{O}_{3}$ minima 
in locations of high NOx emissions. The maxima were underpredicted due to the dilution of precursors. Minima were overpredicted as the NO titration that occurs in NOx inhibited conditions (depleting $\mathrm{O}_{3}$ ) was subdued, again due to dilution of the NOx-rich environment. In conclusion, this research suggested that transport is a linear function with respect to grid resolution whereas chemistry is non-linear. As such, it is expected that a non-reactive species should experience the same average transport at a coarse resolution as over the same area at a fine resolution. For reactive species, such as $\mathrm{O}_{3}$, the transport would not be the same between resolutions. Jang, Jeffries, and Tonnesen (1995) followed up to Jang, et al. (1995a) research by further looking into the detailed impact of grid resolution on $\mathrm{O}_{3}$ chemistry. They found that $\mathrm{NO}_{x}$ related chemistry was more important than VOC related chemistry. They also concluded that vertical transport processes that act on NOx highly influence $\mathrm{O}_{3}$.

Gillani and Pleim (1996) also examined the impact of Eulerian model grid resolution of $\mathrm{O}_{3}$ precursors. Consistent with the results from Jang, et al. (1995) and Jang, Jeffries, and Tonnesen (1995), they find that coarse grid modelling poorly captures local inhomogeneities of precursor concentrations, leading to errors in $\mathrm{O}_{3}$ concentration predictions and associated sensitivities. They recommend that $\mathrm{O}_{3}$ modelling not exceed horizontal resolutions of $20-30 \mathrm{~km}$.

The literature presented thus far has looked at relatively coarse models, with $20 \mathrm{~km}$ as the finest resolution. There have been more recent studies that have examined the 
influence of grid resolution on more local scales, down to a $1 \mathrm{~km}$ resolution or finer. The consensus of the literature reviewed thus far has been that the finer the resolution, the more accurate the results. This trend is not maintained when looking at very fine resolutions $<20 \mathrm{~km}$. Some studies indicate that finer resolutions do not always result in more accurate model performance for $\mathrm{O}_{3}$ formation (Zhang et al., 2006a, 2006b; Wu et al., 2008a).

Tang (2002) simulated $\mathrm{O}_{3}$ and its precursor species at 1.333, 4 and $20 \mathrm{~km}$, and found that the 1.333 and $4 \mathrm{~km}$ results were similar to each other and had good agreement with monitored observations, but the $20 \mathrm{~km}$ results did not. The main cause of this was that $20 \mathrm{~km}$ resolution diluted NOx emissions, underestimating them near power plants, resulting in overestimated $\mathrm{O}_{3}$ levels. This was especially noticeable at nighttime.

Arunachalam et al. (2006) investigated grid resolution impacts on $\mathrm{O}_{3}$ by comparing 36 , 12 and $4 \mathrm{~km}$ resolutions over North Carolina, using the CTM MAQSIP. They concluded that 8-hour $\mathrm{O}_{3}$ predictions were statistically similar between 4 and $12 \mathrm{~km}$ resolutions ( $<3 \%$ difference, on average), and suggested a $12 \mathrm{~km}$ resolution as sufficient. This study also found that between $4 \mathrm{~km}$ and $36 \mathrm{~km}$, there was a 1-3 ppb difference in 8-hour $\mathrm{O}_{3}$ episodes.

In contrast, numerous other studies have found that strong $\mathrm{O}_{3}$ gradients near large precursor emission sources may not be captured by resolutions of $12 \mathrm{~km}$, and as such, 
$12 \mathrm{~km}$ may not accurately predict $\mathrm{O}_{3}$ concentrations (Kumar \& Russel, 1996; Valeri \& Menut, 2008; Tie et al., 2010; Mathur et al., 2005; Cohan, et al., 2005). Tie, et al. (2010) found that $\mathrm{NOx}$ and $\mathrm{O}_{3}$ surface concentrations calculated from CTMs with resolutions of $3 \mathrm{~km}$ and $6 \mathrm{~km}$ were similar to those measured, at $12 \mathrm{~km}$ resolutions there were slight underestimations, and at $24 \mathrm{~km}$ resolutions there were very different results. This is consistent with Mathur et al. (2005), whom found that $\mathrm{O}_{3}$ was best modelled at $4 \mathrm{~km}$ resolution, compared to $12 \mathrm{~km}$ or $36 \mathrm{~km}$, as this resolution better represents the $\mathrm{O}_{3}$ titration by NOx, especially occurring at nighttime. The results in this study from the 36 $\mathrm{km}$ resolution over-predicted urban $\mathrm{O}_{3}$ peaks.

Grid resolution impacts on sensitivity of $\mathrm{O}_{3}$ to NOx emissions were examined at 36,12 and $4 \mathrm{~km}$ resolutions by Cohan et al (2006) in which it was found that there is an increased $\mathrm{O}_{3}$ production with respect to NOx emissions at finer resolutions. This research also concluded that $12 \mathrm{~km}$ resolution can predict $\mathrm{O}_{3}$ prediction efficiently, but to identify localized areas of NOx inhibition a resolution of $4 \mathrm{~km}$ is required.

In regions of complex terrain and land-use, such as coastal regions, modelling $\mathrm{O}_{3}$ at fine resolutions may be of higher importance. Shrestha et al. (2008) used MM5-CMAQ to model the coastal region of Osaka, Japan, an area influenced by sea breeze and heat island effect, at 9, 3 and $1 \mathrm{~km}$ resolutions. The results indicate far better performance by the $1 \mathrm{~km}$ resolution, better simulating the daily maxima and having better agreement at most observation stations. 
In summary, when it comes to modelling $\mathrm{O}_{3}$ there is not a consensus on a general single resolution to best simulate and represent $\mathrm{O}_{3}$ concentrations. There is general agreement that resolutions over $20 \mathrm{~km}$ are not sufficient to model the non-linearity of $\mathrm{O}_{3}$ chemistry, as localized precursor emissions are not adequately represented. Few studies found that $12 \mathrm{~km}$ was sufficient, while most recommended at least $4 \mathrm{~km}$, and some were even as low as $1 \mathrm{~km}$ in regions of complex terrain.

Relative to the numerous studies analyzing the impacts of grid resolution on $\mathrm{O}_{3}$ and $\mathrm{PM}_{2.5}$, there is limited research that analyzes the impacts of grid resolution directly on $\mathrm{NO}_{2}$ concentrations (Vautard et al., 2009; Valin et al., 2011; Schaap et al., 2015; Tan et al., 2015; Kuik et al., 2016). This may be due to the lack of consensus regarding the associated health impacts due to $\mathrm{NO}_{2}$ exposure. Most studies that investigate $\mathrm{NO}_{2}$ and grid resolution impacts mainly is related to $\mathrm{NOx}$ as a precursor to $\mathrm{O}_{3}$, but as such also gives insight to how $\mathrm{NO}_{2}$ itself is impacted by resolution (e.g. Sillman et al., 1990; Kumar et al., 1994; Gillani et al., 1996; Cohan et al., 2006; Tie et al., 2010).

Valin et al. (2011) used WRF-CHEM to analyze the impacts of resolution on $\mathrm{NO}_{2}$ concentrations over three high emission regions: Los Angeles, San Joaquin Valley, and the Four Corners Power Plants, at 4, 12, 24, 48, and $96 \mathrm{~km}$ grid resolution. In this research, they compared the modelled CTM concentrations to column $\mathrm{NO}_{2}$ concentrations from UV/VIS instruments. They conclude that for Joaquin Valley and 
Four Corners, a model resolution of $4 \mathrm{~km}$ was required to accurately simulate, however, only a model resolution of $12 \mathrm{~km}$ was required in Los Angeles.

Schaap et al. (2015) analyzed the impact of horizontal grid resolution on five different CTMS: CHIMERE, CMAQ, EMEP MSC-W, LOTOS-EUROS and RCG. The resolutions ranged from $7 \times 7 \mathrm{~km}$ resolution up to $56 \times 56 \mathrm{~km}$ resolution, depending on the domain area of analysis. Modelled concentrations were statistically compared with monitored concentrations. At urban monitoring stations, this research found a significant gain between modelled results at $56 \mathrm{~km}$ and $7 \mathrm{~km}$ resolutions for all models, with the largest gains seen between $56 \mathrm{~km}$ and $14 \mathrm{~km}$ resolutions. They also demonstrate that $\mathrm{NO}_{2}$ and PM were more sensitive to model resolution than $\mathrm{O}_{3}$, consistent with results found by Queen and Zhang (2008).

Kuik, et al. (2016) examined the impact of resolution on NOx simulated using WRFCHEM for a 3-month period in summer of 2014 over Berlin at 15, 3 and $1 \mathrm{~km}$ resolutions. This research concludes that a horizontal resolution of $3 \mathrm{~km}$ is best suited to adequately resolve spatial NOx within Berlin, with a $15 \mathrm{~km}$ resolution being too coarse to differentiate between rural and urban gradients, while $1 \mathrm{~km}$ resolution results generally did not improve over those generated at $3 \mathrm{~km}$ resolution.

Tie et al, $(2007,2010)$ analyzed the impacts of grid resolution on the formation of $\mathrm{O}_{3}$, and in doing such, examined the impacts on NOx as its precursor. Tie, et al $(2007,2010)$ 
demonstrate that a resolution of $24 \mathrm{~km}$ is not suitable for simulating NOx in a case study over Mexico City, and a finer resolution should be used. This is consistent with results found by Gillani and Pleim (1996) that conclude horizontal resolutions larger than 20-30 km can not adequately resolve NOx emissions/concentrations. Vautard et al. (2009) also demonstrate that high daily mean concentrations of $\mathrm{NO}_{2}$ were poorly predicted at coarse resolutions greater than $25 \mathrm{~km}$.

Results within the reviewed literature can agree that a model resolution less than $25 \mathrm{~km}$ should be used to adequately model $\mathrm{NO}_{2}$ concentrations. The exact resolution suggested to adequately model $\mathrm{NO}_{2}$ varied from study to study, with some suggesting $12 \mathrm{~km}$ be sufficient (e.g. for L.A. in Valin et al., 2011), while others suggest a resolution as fine as 4 or $3 \mathrm{~km}$ be required to accurately predict $\mathrm{NO}_{2}$ concentrations (e.g. for Kuik et al., 2016 and for Joaquin Valley and Four Corners in Valin et al., 2011). The region of study (land use, meteorology) most likely impacts the required resolution and as such, a single resolution cannot be suggested to model $\mathrm{NO}_{2}$.

There is an abundance of research that demonstrates the impacts of grid resolution on $\mathrm{PM}_{2.5}$ (and $\mathrm{PM}_{10}$ ) concentrations (Tesche et al., 2006; Wu et al., 2008a; Queen \& Zhang, 2008; Matthias, Aulinger \& Quante, 2008; De Meij et al., 2008; Mensink et al., 2008; Ott et al., 2008; Shreshtha et al., 2009; Vautard et al., 2009; Arunachalam et al., 2011; Stroud et al., 2011; ; Fountoukis et al., 2013; Punger \& West, 2013; Schapp et al., 2015). 
The US EPA recommends modelling $\mathrm{PM}_{2.5}$ concentrations at fine resolutions $<12 \mathrm{~km}$, and higher resolution in areas of large emission sources, as fine as $4 \mathrm{~km}$ (US EPA, 2007).

Fine $\mathrm{PM}_{2.5}$ is composed of many different species, with atmospheric lifetimes that range from days to weeks (Seinfeld \& Pandis, 2006). The short-lived components have short dispersal ranges and high concentrations close to emission source. Ott et al. (2008) demonstrated that PM concentrations were heterogeneous over short distances by monitoring PM at a series of stations with an average of $4.4 \mathrm{~km}$ between sites. These spatial gradients are not well captured by models with coarse resolutions.

Punger \& West (2013) used CMAQ to study the impacts of resolution on $\mathrm{PM}_{2.5}$ concentrations and associated exposure impacts, from $12 \mathrm{~km}$ resolutions up to $408 \mathrm{~km}$ resolution. At resolutions $<100 \mathrm{~km}$, the coarse concentration results were $<20 \%$ lower than those generated at $12 \mathrm{~km}$ resolution, at resolutions between $100-250 \mathrm{~km}$, coarse concentrations were $20-30 \%$ lower than at $12 \mathrm{~km}$ resolution, and at resolutions $>250$ $\mathrm{km}$, concentration were $30-40 \%$ lower than at $12 \mathrm{~km}$ resolution. These results indicate that coarse grid resolutions poorly capture local $\mathrm{PM}_{2.5}$ gradients and found the largest discrepancies in urban regions. Punger and West (2013) also found that the maximum $\mathrm{PM}_{2.5}$ concentration decreased by $26 \%$ from $12 \mathrm{~km}$ to 36 resolution, while it decreased by $71 \%$ from $12 \mathrm{~km}$ to $408 \mathrm{~km}$ resolution. 
Fountoukis et al. (2013) studied the impacts of grid resolution on fine PM using PMCAMx. This research had two separate cases: one over the Northeastern U.S. at resolutions of 12 and $36 \mathrm{~km}$ in which the $12 \mathrm{~km}$ emission inventory was interpolated from the $36 \mathrm{~km}$, the other over Western Europe at resolutions of 4 and $36 \mathrm{~km}$, in which the $4 \mathrm{~km}$ emission inventory was developed with high resolution. The results showed higher influence due to resolution during the winter than the summer, and higher discrepancies were mainly related to primary species than secondary. They found that primary PM Species (organics and black carbon) were more sensitive to resolution than secondary (e.g. sulfate), with the bias for black carbon decreasing more than $30 \%$ in the winter at $4 \mathrm{~km}$ resolution compared to the $36 \mathrm{~km}$ resolution. Overall, this research found improvements with model accuracy and high resolution.

In Schaap et al.'s (2015) research (introduced in Section 2.3.1.2) evaluating impacts of resolution using multiple $\mathrm{CTMs}, \mathrm{PM}_{10}$ was a species of investigation. In urban locations, they find that between $56 \mathrm{~km}$ and $7 \mathrm{~km}$, the spatial bias is reduced for all models when compared to monitored concentrations. Rural locations had a slight decrease in modeled concentrations at higher resolution, bringing the values closer to those monitored.

Wu et al. (2008a) compared CMAQ results generated at 36, 12 and $4 \mathrm{~km}$ resolutions to monitored results. This research found improved model performance as the resolution got finer. Overall, 12 and $4 \mathrm{~km}$ resolution results were not very different, but 4 to 36 had 
a large impact, which is contrasting to some of the previously reviewed studies which concluded $4 \mathrm{~km}$ may not be fine enough.

Tan et al. (2015) used WRF/CMAQ model at $3 \mathrm{~km}$ and $1 \mathrm{~km}$ resolutions to examine grid resolution impacts at fine resolutions and comparing the performance with ground-level monitored concentrations. The $\mathrm{PM}_{10}$ results of this study demonstrate that the fine resolution $(1 \mathrm{~km})$ results had better agreement with monitored concentrations, with the relative error ranging from $-25 \%$ to $59 \%$, compared to the $3 \mathrm{~km}$ results that had relative errors from $26 \%$ to $245 \%$.

Improvements in modelled PM concentrations occur with increasing resolutions, with studies finding that even $4 \mathrm{~km}$ may not be fine enough to accurately predict PM concentrations (Mensink et al., 2008; Ott et al., 2008, Shreshtha et al., 2009; Tan et al., 2015). In contrast, Tesche et al. (2006) found no significant difference in $\mathrm{PM}_{2.5}$ concentrations between 12 and $36 \mathrm{~km}$, a finding consistent with Wu et al. (2008). There is not a consensus of a single resolution in which PM should be modelled, the impacts most likely depends on the location of analysis and the corresponding land-use and meteorology.

\subsubsection{Effects of CTM Grid Resolution on Health Impact Predictions}

Influences due to the resolution of CTMs are carried through onto the predicted associated health impacts, a function of both the CTM concentration outputs and population. While there are numerous studies that have analyzed the influence of 
model resolution on pollutant concentrations (refer to Section 2.3.1) there are fewer that have examined its influence on human health impacts (Arunachalam et al., 2011; Thompson and Selin, 2012; Punger \& West, 2013; Thompson et al., 2014; Li et al., 2015). This section reviews the studies that have focused on the impacts of CTM resolution on associated health impacts related to $\mathrm{O}_{3}, \mathrm{NO}_{2}$ and $\mathrm{PM}_{2.5}$ exposure.

Punger and West (2013) assessed mortality estimates related to $\mathrm{PM}_{2.5}$ and $\mathrm{O}_{3}$ at model resolutions between $12-408 \mathrm{~km}$ and found that at coarse resolutions, $\mathrm{PM}_{2.5}$ related mortality estimates were significantly low (30-40\% lower at $>250 \mathrm{~km}$ than at $12 \mathrm{~km}$ ) but $\mathrm{O}_{3}$ only showed a maximum of $6 \%$ difference in estimated mortality between coarse and fine resolutions. Punger and West (2013) also estimated the total U.S.-wide mortality associated with $\mathrm{O}_{3}$ exposure and found that at $36 \mathrm{~km}$, there is a $12 \%$ higher mortality burden than at $12 \mathrm{~km}$. This research also found that pollutants with short atmospheric lifetimes, such as $\mathrm{PM}_{2.5}$, vary more drastically spatially, causing more of a bias due to resolution than found for $\mathrm{O}_{3}$. This was especially true for primary $\mathrm{PM}_{2.5}$ which has a short dispersal range.

Thompson and Selin (2012) studied health impacts due to $\mathrm{O}_{3}$ exposure simulated at 36, 12,4 and $2 \mathrm{~km}$, comparing the concentration results against daily maximum 8-hour average $\mathrm{O}_{3}$ from monitoring stations. They found the concentrations were more consistent between the different model iterations than the monitored values. Health impacts (avoided mortalities) were not significantly different between 2, 4, and $12 \mathrm{~km}$ 
resolutions, but may have been overestimated at $36 \mathrm{~km}$. They conclude with suggesting that $12 \mathrm{~km}$ resolution may be appropriate to estimate health benefits due to $\mathrm{O}_{3}$ reductions, however, it may change on a case-by-case basis.

Thompson, Saari and Selin (2014) evaluated CTM resolution on human health impacts resulting from changes to $\mathrm{O}_{3}$ and $\mathrm{PM}_{2.5}$ concentrations. This research used CAMx paired with BenMAP to estimate human health impacts due to changes in concentrations modelled over resolutions of resolutions of 36,12 and $4 \mathrm{~km}$. Health impacts due to changes in concentrations of $\mathrm{O}_{3}$ were sensitive to model resolution, however in contrast, health impacts due to changes in concentration of $\mathrm{PM}_{2.5}$ were not sensitive to resolution. This study concludes that when looking at resolution influence on the health response to change in total $\mathrm{PM}_{2.5}$ and $\mathrm{O}_{3}$ (combined) concentrations, there was no difference between $36 \mathrm{~km}$ results and those generated at fine resolutions, a result due to $\mathrm{PM}_{2.5}$ dominated health impacts.

Li et al. (2015) also looked at the influence of CTM resolution on health impacts for $\mathrm{PM}_{2.5}$ and found that at a coarse resolution $\left(2 \times 2.5^{\circ}\right)$, the national (USA) mortality was $8 \%$ lower than the fine-model $\left(0.5 \times 0.66^{\circ}\right)$ estimate. Thompson et al. $(2014)$ found that the magnitude of health benefits associated with primary $\mathrm{PM}_{2.5}$ increased about $22 \%$ between $36 \mathrm{~km}$ and $4 \mathrm{~km}$, indicating that the coarse grid (36 km) underestimated the health impacts. Li et al. (2015) and Thompson et al. (2014) similarly found that health impacts due to $\mathrm{PM}_{2.5}$ exposure were underestimated at coarse resolutions. In the same 
study by Thompson et al. (2014), secondary $\mathrm{PM}_{2.5}$ concentrations and health impacts did not show a strong correlation with model resolution.

Arunachalam et al. (2011) evaluated the importance of model resolution on $\mathrm{PM}_{2.5}$ exposure related health impacts (mortality) using CMAQ at resolutions of 36, 12, and 4 $\mathrm{km}$. They found that although maximum concentrations varied drastically between resolutions, aggregated total health risks over the entire mode domain did not change significantly between resolutions with only a $2 \%$ mortality difference between 36 and 12 $\mathrm{km}$. The 4-km resolution had lower overall domain-wide health risk estimates.

Research thus far has contradicting conclusions about the influence of model resolution on estimated exposure related heath impacts. Some studies conclude that $\mathrm{O}_{3}$ and/or $\mathrm{PM}_{2.5}$ health impacts predicted from fine resolution modelling $(<36 \mathrm{~km})$ are not more accurate than those predicted from coarse resolution modelling (>36 km) (Arunachalam et al., 2011; Thompson and Selin, 2012; Thompson et al. 2014), while other studies (Punger and West, 2013) found that coarse resolutions (36 km) had higher predicted health impacts for $\mathrm{O}_{3}$ and lower for $\mathrm{PM}_{2.5}$ than results generated at fine $(12 \mathrm{~km})$ resolutions. There was a lack of studies related to model resolution and $\mathrm{NO}_{2}$ related health impacts throughout this review. 


\subsubsection{Impacts from Meteorological Inputs Resolution}

In CTMs, meteorological fields drive the transportation of pollutants causing their concentrations to be highly sensitive to meteorological inputs. CTMs further rely on the meteorological inputs for aerosol-cloud chemistry, for photochemical reactions (a function of solar radiance and cloud cover), temperature dependent chemistry, for landuse parameters, and for the deposition of species within the model. Several studies have found that the accuracy of CTMs is impacted by the horizontal resolution of the input meteorological data (Baklanov et al., 2002; Fenger, 2003; Menut, Coll \& Cautenet, 2005; Palau et al., 2005; Jimenez et al., 2006; Queen and Zhang, 2008; Wu et al., 2008a; Wolke et al., 2012;). The resolution of meteorology has stronger influences on CTMs in coastal regions or those with complex terrain (e.g. mountain, valley) than in simple terrain areas due to the strong spatial gradients. Palau et al. (2005) and Fenger (2003) suggest that for complex terrain coastal regions, lower layer pollution behaviour can be poorly simulated due to the strong influence resolution has on integral advection and turbulent dispersion.

There is an abundance of research that has evaluated the impact of resolution on meteorological model results, but they often do not pair these with CTMs (Weisman et al., 1997; Grabowski et al., 1998; Stohl et al., 1995; Gallus, 1999; Mass, Ovens \& Westrick, 2002; Colle, Olson \& Tongue, 2003; Leung \& Qian, 2005; Gao et al., 2006; Rauscher et al., 2009; Ramu et al., 2016). This section does not focus on these studies but will refer to them when direct impacts on CTMs are drawn. 
Concentrations of pollutants will be heavily affected by errors in convective precipitation, a process resulting in pollutant scavenging. Coarse resolution modelling can result in significant errors in heavy precipitation events (Weisman et al., 1997; Grabowski et al., 1998, Mass, Ovens \& Westrick, 2002; Leung \& Qian, 2002;) and slower convection evolution (Weisman et al., 1997; Mass, Ovens \& Westrick, 2002), factors which influence the modeling of precipitation. Heavy precipitation can be diluted at coarse resolution modelling, which consequently will overestimate precipitation in nearby areas. Other meteorological fields have been shown to be influenced by horizontal resolution, such as wind fields and the planetary boundary layer, both parameters that directly impact air quality through transportation (Baklanov et al., 2002; Jimenez et al., 2005).

Wu et al. (2008a) used MM5/CMAQ at various resolutions (36, 12 and $4 \mathrm{~km}$ ) to evaluate the impact on meteorological parameters and on concentration predictions. Wu et al. (2008a and 2008b) found that pollutant concentrations of $\mathrm{PM}_{2.5}, \mathrm{SO}_{4}{ }^{2-}$ and $\mathrm{NO}_{3}{ }^{-}$had higher sensitivity to the grid resolution of meteorology (cloud fractions and precipitation) than compared to those of emissions. They also found high model performance at $4 \mathrm{~km}$ resolution for meteorological variables and $\mathrm{O}_{3}$ mixing ratios, showing better performance than at $12 \mathrm{~km}$ and $36 \mathrm{~km}$ resolutions. However, minimum overnight temperatures in August were overpredicted and relative humidity was underpredicted in both summer and winter months. 
Menut, Coll and Cautenet (2005) studied how the resolution of meteorological data impacted $\mathrm{O}_{3}$ concentrations modelled using CHIMERE. This study looked at two cases, one with meteorology processed using ECMW at $40 \mathrm{~km}$ resolution and one with meteorology using RAMS at $3 \mathrm{~km}$ resolution. They found impacts due to resolution on the flow field properties: wind components, temperature, pressure and humidity, as well as indirect impacts to the vertical diffusivity, friction velocity, and the boundary layer depth. The fine resolution case ultimately had better simulation of local winds and better nighttime $\mathrm{O}_{3}$ predictions, but both cases had decent simulation of $\mathrm{O}_{3}$ when compared to monitored values.

Queen and Zhang (2008) simulated $\mathrm{O}_{3}$ and $\mathrm{PM}_{2.5}$ at resolutions of $4 \mathrm{~km}, 12 \mathrm{~km}$, and 36 km using MM5-CMAQ while also focusing on the impact of resolution on meteorological conditions such as precipitation and wet deposition. They find that at the $4 \mathrm{~km}$ resolution, results were the best for predicting mesoscale precipitation and wet deposition amounts. $\mathrm{O}_{3}$ simulated results were also most realistic at $4 \mathrm{~km}$. Their results also demonstrated that the influence of horizontal resolution on meteorology depended on the region (e.g. terrain type) and on the time of year (e.g. summer or winter).

Mass, Ovens and Westrick (2002) found considerable improvements in temperature and wind predictions when grid resolution decreased from $36 \mathrm{~km}$ to $12 \mathrm{~km}$ using the MM5 model, however, only minor improvements when further reducing to $4 \mathrm{~km}$. The same 
study by Mass, Ovens \& Westrick (2002) demonstrated that at coastal areas, $4 \mathrm{~km}$ wind estimates were superior.

In summary, research demonstrates that meteorological fields are impacted by the horizontal resolution and that CTMs are impacted by the resolution of the input meteorological data. Coarse resolution meteorology inputs may not be able to sufficiently delineate the fields that impact pollution transport, scavenging, and reaction rates.

\subsubsection{Impacts from Emission Inputs Resolution}

Fine resolutions are required to capture the strong spatial gradients of emissions that occur in urban and surrounding suburban area. In CTMs, primary emissions are averaged instantaneously and uniformly across each grid cell volume, causing artificial dilution. Artificial dilution is especially significant at coarse resolutions over urban regions or near large emission sources, where these emissions are strongly inhomogeneous. This directly impacts the concentrations of primary species, such as $\mathrm{NO}_{2}$ and some $\mathrm{PM}_{2.5}$ species, and precursors of secondary species, such as NOx and VOCs for the formation of $\mathrm{O}_{3}$. The high non-linearity chemistry of $\mathrm{O}_{3}$ and its sensitivity to precursors causes inaccurate representation if precursor emissions are coarse. Coarse model emissions cannot sufficiently simulate the extent of NO-titration of $\mathrm{O}_{3}$ in urban areas (Jang et al., 1995; Im \& Kanakidou, 2012). 
Hodnebrog, Stordal and Berntsen (2011) used WRF-CHEM to analyze the impacts of emission resolution on modelled $\mathrm{O}_{3}$ concentrations in three megacities, Ruhr, London and Cairo. In this study, the CTM resolution was held constant at $9 \mathrm{~km}$, while the resolution of the emission inventories varied between 9,27 , and $81 \mathrm{~km}$. Their results show that at the coarse emission inventory, net $\mathrm{O}_{3}$ was $12 \%$ higher than at the finest emission inventory. This could be due to the dilution of NOx emissions to NOx limited regions in the coarse scenario, causing lower $\mathrm{NO}$-titration of $\mathrm{O}_{3}$, similar to conclusions by Jang et al. (1995) and Im and Kanakidou (2012). Overall, the differences were generally small between changing emissions from $9 \mathrm{~km}$ to $81 \mathrm{~km}$ resolutions.

Wu et al. (2008a \& 2008b) found that pollutant concentrations of $\mathrm{PM}_{2.5}, \mathrm{SO}_{4}{ }^{2-}$ and $\mathrm{NO}_{3}{ }^{-}$ were less sensitive to the resolution of emissions than they were to the resolution of meteorology.

Jang et al. (1995) found that CTMs with $80 \mathrm{~km}$ resolution could not resolve NOx and VOC emissions, which resulted in $\mathrm{O}_{3}$ being underpredicted downwind of emission sources and overpredicted in locations of high NOx emissions. The maxima were underpredicted due to the dilution of precursors. Minima were overpredicted as the NO titration that occurs in NOx inhibited conditions (depleting $\mathrm{O}_{3}$ ) was underpredicted.

Cohan et al. (2006) evaluated the extent of influence that model resolution has on the sensitivity of ground-level $\mathrm{O}_{3}$ to precursors (NOx and VOCs). They found relatively similar predictions at all resolutions with respect to NOx emissions, but a resolution of 
$36 \mathrm{~km}$ was insufficient to capture sensitivities with respect to VOCs, resulting in underpredictions in $\mathrm{O}_{3}$.

\subsubsection{Trade-offs of Fine Resolution Modelling}

Despite plenty of evidence indicating that fine resolution modelling improves the accuracy and spatial representation of results, it comes at an associated cost, creating a trade-off between accuracy and spatial representation and efficiency. The computational cost significantly increases as model resolution becomes finer. Each halving of the horizontal grid dimension results in quadruple grid cells to cover the given domain. This results in significant increase in computation time for integration of the equations that drive the model, i.e., the atmospheric diffusion equation (ADE).

Additional to the number of grid cells, at fine resolutions, shorter time steps are required to satisfy stability constraints, resulting in more time steps required for a given period compared to a coarse model. This further increases the amount of computations required to run the simulation, further increasing the modelling time.

Another trade-off of fine resolution modelling is that both input and output file sizes are greater in size, as there are more results saved for the greater amount of time steps and grid cells. This requires a high quantity of storage. The costs to process fine resolution inputs (e.g. meteorology and emissions) can also be heightened at fine resolutions, accumulating to the total cost of running air quality models at fine resolution. 
Additionally, there is higher effort involved in developing fine resolution inputs, such as emissions, as the fine resolution data may not be readily available. The difficulty of simulating such fine scale inputs can result in higher uncertainties within the data.

Due to these trade-offs, it is not always feasible to run CTMs at fine resolutions. The application of the results must be considered prior to deciding whether there is justification for fine resolution modelling. If one wants to look at the impacts of an air quality management policy from a specific emission source (e.g. power plant), then fine grid sensitivity modelling may be beneficial as it can allow specific source contributions to be narrowed down to the individual emission source. Fine grid modelling can also be beneficial in urban planning and development, as one can determine suitable locations to build sensitive facilities such as nursing homes, or to determine the impacts of developing emission sources at specific locations. 


\subsection{Methods}

Within this thesis, human health impacts are predicted by pairing the sensitivity analysis of atmospheric CTMs with pollution exposure equations. Through multiple iterations of various model and input resolution scenarios, the overall impact of grid resolution on these health estimates is analyzed.

This section outlines the basics principles of the CTM used to perform the sensitivity analysis, and their pairing with epidemiology to estimate health impacts. It is intended to act as a basis of fundamentals, while chapters 4 and 5 contain detailed methodology as part of the draft manuscripts.

\subsection{The CMAQ Model}

The research performed within this thesis uses the 3-D Eulerian CTM model known as the Community Multi-Scale Air Quality (CMAQ) model (Byun \& Schere, 2006). CMAQ is an open-source CTM that was developed and is actively being revised and refined by the U.S. EPA, incorporating input from a large international user community. CMAQ is used for a multitude of applications, such as pollution forecasting, policy development and assessment, consulting, and research.

Inputs to the CMAQ model include meteorology, emissions, initial conditions and boundary conditions. There are several preparatory and science processes that make up the requirements of the CMAQ modelling system which are displayed in Figure 3-1. 
CMAQ computationally solves the ADE (introduced in Equations 2.1 and 2.2), within each direction ( $x$ and $y$ horizontal directions, $z$ vertical) and throughout time, as displayed in Figure 3-1 by the "Continuity Equation."

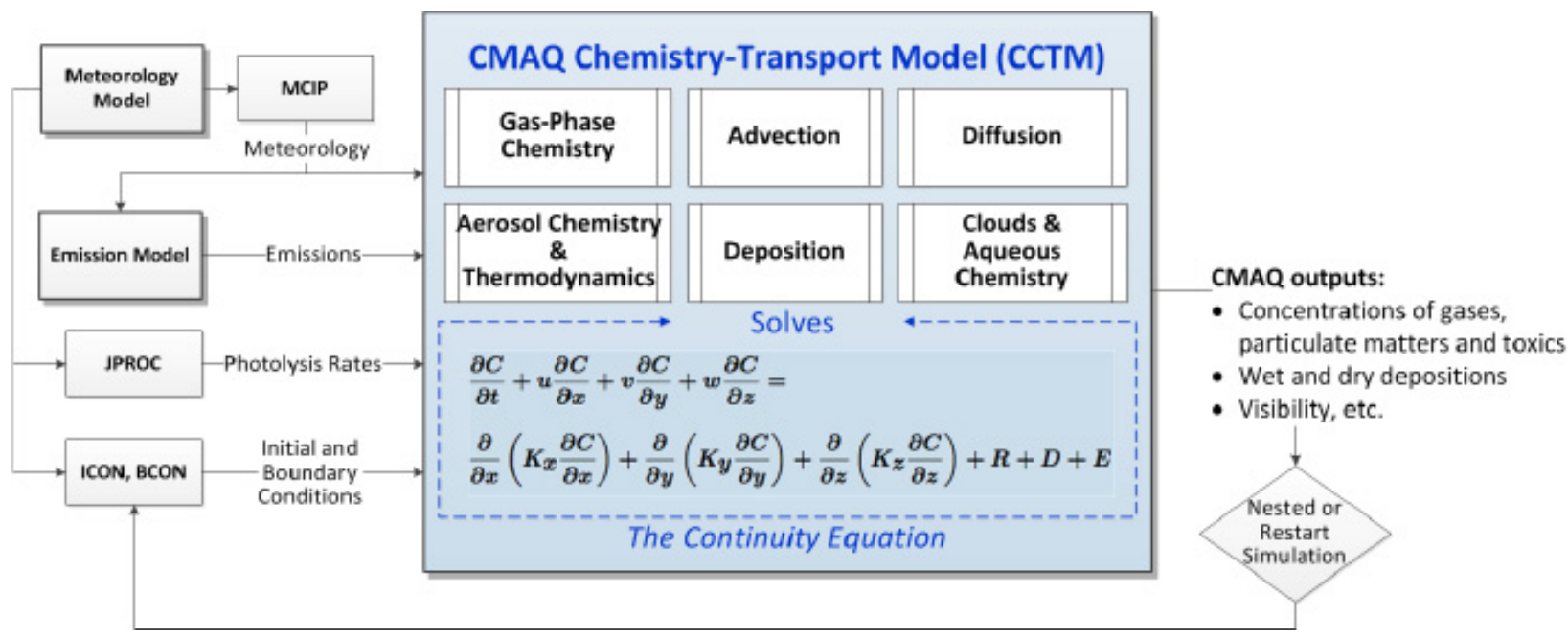

Figure 3-1. The CMAQ modelling system and required processors (adapted from Washington State University, 2018).

The initial and boundary conditions are developed using the Initial Conditions Processor (ICON) and the Boundary Conditions Processor (BCON), respectively, which are both software applications of the CMAQ modelling package, Models-3. Meteorology and emission inputs are processed using independent software programs. The meteorological inputs used in this thesis were developed using the Weather, Research and Forecasting (WRF) model (National Center for Atmospheric Research, 2018), and were processed into a format able to be inputted into CMAQ using the MeteorologyChemistry Interface Processor (MCIP). A collaboration was established with modellers from Rowan Williams Davies and Irwan Inc. (RWDI), who developed the 2012 emission 
inventory for each resolution. The United States emissions were sourced from the National Emission Inventory (NEI). Canadian emissions were sourced from Environment and Climate Change Canada's (ECCC) 2010 National Pollution Release Inventory (NPRI) for point sources, the 2011 National Agri-Environmental Standards Initiative for area sources, and the remaining area and mobile emissions from EC's 2006 Criteria Area Contaminants (CAC). Emissions were then processed into a format that could be inputted into CMAQ using the Sparse Matrix Operator Kernel for Emissions (SMOKE).

\subsection{Sensitivity Analysis}

Sensitivity analysis is used to describe the change, or gradient, between computational model inputs and their impacts on model outputs. In the sense of atmospheric CTMs, sensitivities are frequently used to demonstrate how the receptor concentrations change due to changes in emission sources or atmospheric parameters, as described by the following Equation 3.1 (Yang et al, 1997):

$$
s_{i, j}(t)=\frac{\partial c_{i}(t)}{\partial p_{j}}
$$

Where: $p_{j}$ is the model parameter or input

$C_{i}$ is the concentration of species $i$

The above Equation 3.1 describes the derivative of model outputs ( $C$, concentration) with respect to model inputs ( $p$, model parameter such as emissions). 
The effectiveness of air quality management policies can be analysed using sensitivity modelling as it can demonstrate how anthropogenic emission reductions (or increases) will impact pollutant concentrations. This can further be applied to estimate impacts on public health or other environmental concerns. Sensitivity modelling allows for the targeting of specific emission reductions, by location or species, to create the most beneficial air quality management policies.

There are two main categories of sensitivity analysis, forward sensitivity and backward sensitivity. Forward sensitivity models start with one input (e.g. an emission source in a specific location) and estimate how its change will impact model outputs at all model receptors (e.g. concentrations across the entire domain and at all time steps). Forward sensitivity modelling can be described as "source based." Some of the most commonly used formal forward sensitivity methods are brute-force (finite difference) and the decoupled direct method (DDM). Forward sensitivity models are limited to estimating sensitivity from a single input parameter or a single collection of inputs. If it is desirable to investigate the influence of multiple input parameters, one must run the sensitivity analysis with respect to each input, which can quickly become inefficient and cumbersome.

Backward sensitivity models allow for the impacts from multiple input sources (e.g. emission reductions in every grid cell within the modelling domain) on changes at one output receptor or a group of receptors (e.g. average concentration over the entire 
domain). Backward sensitivity modelling can be described as "receptor based."

Backward sensitivity methods rely on employing an adjoint version of the underlying model, such as the model used within this thesis and described in further detail in Section 3.2.3. Backward sensitivity is an efficient method when the impacts of multiple inputs are required, where using a forward sensitivity model would become cumbersome.

Whether to use a forward or backward sensitivity model depends on the intended application of the results. If one is interested in the impacts of a specific source (e.g. a power plant), on all receptors then a forward method is the best option. If one is interested in the impact from all of the sources on one receptor (e.g. a city), then a backward method is the best option. Within this section, some of the most common sensitivity analysis approaches used in CTMs are described, focusing in depth on the adjoint sensitivity analysis, the model used to perform the work within this thesis.

\subsubsection{Forward Sensitivity Analysis: Brute Force Method}

The brute-force (BF) method is a forward sensitivity finite-difference technique which involves making small perturbations to a model input or a single parameter, while holding other parameters constant, and investigating the associated change to the output. This forward sensitivity approximation method is simple to implement, however, entails high computational costs for a larger number of sensitivity parameters and is inefficient as a complete run is required for the sensitivity to each coefficient to 
be determined (Yarwood et al., 2007). This makes it unsuitable for determining sensitivities to a large number of model parameters.

Brute-force works by establishing a base case or control case, the following example estimates the base concentration, $C_{0}$, with respect to the base parameter of emissions, $\mathrm{E}_{0}$ :

$$
C_{0}=C\left(E_{0}\right)
$$

Then the concentration is evaluated for a perturbed emission value, $E_{f}$, in which $E_{f}$ is different than $E_{0}$ by a fractional change of $\Delta f$ :

$$
\begin{gathered}
E_{f}=(1+\Delta f) E_{0} \\
C_{f}=C\left(E_{f}\right)
\end{gathered}
$$

The linear slope found from the concentration difference between the base and perturbed simulations defines the first order sensitivity, Ss:

$$
S \cong \frac{C\left(E_{f}\right)-C\left(E_{o}\right)}{\Delta f}
$$

Pollutants that have non-linear reactions within the atmosphere will not be accurately approximated by the brute-force method, especially as $\Delta f$ increases. 


\subsubsection{Forward Sensitivity Analysis: Decoupled Direct Method}

The decoupled direct method (DDM), also referred to as the tangent linear model (TLM), is another formal forward sensitivity technique. DDM derives sensitivity equations from underlying model equations. DDM equations will then represent infinitesimal changes to a parameter. DDM allows multiple perpetrations to be established simultaneously, opposed to just one such as within the BF method.

DDM numerically solves the ADE (Equation 2.2) through operator splitting to determine the sensitivities. As previously introduced, the local sensitivity can be determined as the partial derivative of the output with respect to the input or parameter (Yang, 1997):

$$
S_{i, j}(t)=\frac{\partial c_{i}(t)}{\partial p_{j}}
$$

Sensitivity coefficients can vary by several orders of magnitude due to the model parameters and inputs ranging spatiotemporally (Yang, 1997). Sensitivity coefficients are thus semi-normalized by the partial derivative of a species concentration with respect to the scaling variable of the parameter (Yang, 1997):

$$
S_{i j}^{*}(t)=P_{j} \frac{\partial c_{i}(t)}{\partial p_{j}}=P_{j} \frac{\partial c_{i}(t)}{\partial\left(\epsilon_{j} P_{j}\right)}=\frac{\partial c_{i}(t)}{\partial \epsilon_{j}}
$$

Where ${ }^{*}{ }_{i j}(t)$ is the semi-normalized sensitivity coefficient

$P_{j}(x, t)$ is the unperturbed field

$\epsilon_{\mathrm{j}}$ is a scaling variable with 1 as the nominal value 
By substituting Equation 3.7 into the $A D E$ (Equation 2.2), the supporting sensitivity coefficients are found:

$\frac{\partial s_{i j}^{*}}{\partial t}=-\nabla\left(u s_{i j}^{*}\right)+\nabla\left(K \nabla s_{i j}^{*}\right)+J_{i k} s_{k j}^{*}+\frac{\partial R_{i}}{\partial \epsilon_{j}}+\frac{\partial s_{i}}{\partial \epsilon_{i}}-\nabla\left(u c_{i}\right) \delta_{i j}+\nabla\left(K \nabla c_{i}\right) \delta_{i j}$

Where $\mathrm{J}$ is the Jacobian matrix $\left(J_{i k}=\frac{\partial R}{\partial c_{k}}\right)$

$\delta_{i j}$ is the Kronecker delta function

The following Equation 3.9 is a simplified version of Equation 3.8 which equates the sensitivity of concentration, $\delta \mathrm{C}_{\mathrm{i}}$, with respect specifically to a change in emissions $\delta \mathrm{E}_{\mathrm{i}}$ (Hakami et al., 2007):

$$
\frac{\partial \delta C_{i}}{\partial t}=-\nabla \cdot\left(u \delta C_{i}\right)+\frac{1}{p} \nabla \cdot\left(p K \nabla C_{i}\right)+F_{i} \delta C+\delta E_{i}
$$

Where $\delta \mathrm{C}$ represents the change in concentration of species $i$

$p$ is the density

$F_{i}$ is the $i^{\text {th }}$ row of the Jacobian matrix of the non-linear chemistry transformation

$\delta$ E represents an emission perturbation

\subsubsection{Adjoint (backward) Sensitivity Analysis}

Adjoint is a backwards sensitivity approach that computes the gradient of a single output with respect to the multiple model inputs and parameters (Sandu et al., 2005). Adjoint is an efficient sensitivity method for receptor-based analysis, beneficial if one is interested in estimating how model outputs are influenced by numerous model inputs. Adjoint sensitivity is a reverse approach to forward sensitivity, operating by integrating 
perturbation impacts backward in time and space through a series of sensitivity equations auxiliary to the forward sensitivity.

A metric which relates model outputs to model inputs (i.e. sensitivities), known as the "cost function" is what the adjoint analysis depends on. For AQM, the adjoint cost function is any metric relying on concentration, integrated spatially $(\omega)$ and temporally (t), as displayed in the following Equation 3.10:

$$
J=\int_{t} \int_{\omega} f(C, t, \omega) d \omega d t
$$

The derivative of the cost function with respect to concentration gives the adjoint variable, $\lambda_{\mathrm{i}}$, for species $i$ :

$$
\lambda_{i}=\frac{\partial J}{\partial C_{i}}
$$

By applying Langrangian multipliers to Equation 3.9, and integrating by parts while considering initial and boundary conditions, the following governing adjoint equation is derived (Equation 3.12) (Sandu et al., 2005):

$$
\frac{\partial \lambda_{i}}{\partial t}=-u \cdot \nabla \lambda_{i}-\nabla \cdot\left(\rho K \nabla \frac{\lambda_{i}}{\rho}\right)-F_{i}^{T} \lambda-\varphi_{i}
$$

Where $F_{i}^{T}$ is the $\mathrm{i}^{\text {th }}$ row of the transpose Jacobian matrix for the chemical reaction operator, F

$\lambda_{i}$ defines the adjoint variable specific for time and location $\varphi_{i}$ is the forcing term which drives the adjoint sensitivity over time and is the derivative of the local cost function with respect to concentration $\left(\frac{\partial J}{\partial c_{i}}\right)$. 
The adjoint sensitivity equation has a negative sign, resulting in the backward integration from receptor to source by the reversal of wind fields (Hakami et al, 2007). Adjoint forcing terms, $\varphi$, which are dependent on the definition of the adjoint cost function, are used to drive the sensitivity of the adjoint model as defined in Equation 3.12. The adjoint forcing term, $\varphi$, is defined as the derivative of the cost function (Equation 3.10) with respect to concentration:

$$
\varphi_{i}=\frac{\partial f}{\partial C_{i}}
$$

Adjoint is a formal sensitivity method, which means that it solves the derivative of the equations. It can be used to determine the sensitivity of a limited number of model outputs to numerous model parameters or inputs. The adjoint method is an efficient tool for receptor-based analysis because it works backwards with respect to emission sources. The adjoint sensitivity model developed for CMAQ is used throughout this thesis.

\subsection{Estimation of Health Impacts}

To estimate health impacts using adjoint sensitivity, first an adjoint cost function (M) must be established that relates concentration to the health metric in which the sensitivities are being estimated for. This thesis focuses on the monetized value of mortality due to long-term exposure to air pollution, specifically to $\mathrm{O}_{3}, \mathrm{NO}_{2}$ and $\mathrm{PM}_{2.5}$. Adjoint sensitivity analysis has been previously applied to estimate monetized health 
impacts from pollution exposure (Pappin \& Hakami, 2013a, 2013b; Pappin et al., 2015b, 2016). The cost function applied within this thesis describes the change in monetized mortality valuation $(\Delta M)$ resulting from a change in pollutant concentration $(\Delta C)$ and is expressed by the following Equation 3.14:

$$
\Delta M(\$)=M_{0} \times P \times V_{S L}\left(1-e^{-\beta \Delta C}\right)
$$

Where $M_{0}$ is the baseline nonaccidental mortality rate

$P$ is the population

$V_{S L}$ is the value of statistical life

$B$ is the concentration response factor (CRF)

The $V_{S L}$ is an economic valuation of a statistical life, based off the amount one is willing to pay to reduce the probability of death. It is frequently represented as an averaged singular value, with the most recent Canadian $V_{S L}$ valuing at $\$ 7.17 \mathrm{M}$ in $2013 \mathrm{CAD}$ (Chestnut \& Civita, 2009). The concentration-response function (CRF) varies depending on the epidemiology scenario being studied, whether related to short-term or long-term (chronic) exposure, morbidity or mortality, all-cause or non-accidental, and/or impacts relating to specific disease (e.g. cardiovascular), and it is also dependent on the species being analyzed. Within this research, the CRFs applied were related to non-accidental mortality due to chronic exposure to $\mathrm{O}_{3}, \mathrm{NO}_{2}$, and $\mathrm{PM}_{2.5}$, for a population 25 years of age and above. The CRFs applied were: $0.27 \%$ change in mortality (non-accidental) /change in ppb of daily maximum 8 -hour average $\mathrm{O}_{3}$ (DM8A), $0.59 \%$ change in mortality (non-accidental) /ppb change $\mathrm{NO}_{2}$ (daily average), and $2.7 \%$ change in mortality (nonaccidental) $/ \mu \mathrm{g} / \mathrm{m}^{3}$ change in 24-hour average $\mathrm{PM}_{2.5}$ concentration (Crouse et al., 2015). 
The baseline mortality rates (BMR) (for non-accidental mortality, aged twenty-five plus) were obtained from Health Canada's Air Quality Benefits Assessment Tool (AQBAT) (Judek et al., 2006) for each Census Division. The BMR data was population weighted and then gridded to the respective resolution using CMAQ's spatial allocator tool. Population data was obtained from Statistics Canada (Census Dissemination Area) from the 2011 Census and included only the population aged twenty-five and above to match the CRF and BMR demographics. Population was also aggregated to the respective resolution using spatial allocator.

What is known as the forcing term is used to drive adjoint sensitivity analysis. The forcing term is the derivative of the cost function (Equation 3.10), the forcing term in this research by differentiating Equation 3.15:

$$
\phi=\frac{\partial M}{\delta C}=\frac{V_{S L} M_{0} P \beta e^{-\beta C}}{t n}
$$

Where $\varphi$ is the forcing term

$t$ is the hours in the exposure metric (i.e. 8 hours for $\mathrm{O}_{3}$; 24 hours for $\mathrm{NO}_{2}, 24$ hours for $\mathrm{PM}_{2.5}$ )

$\mathrm{n}$ is the number of days in the period of simulation (365 in this case as all results are expressed as "annually")

The remaining variables on the right-side have the same definitions as in Equation 3.14 . 
This forcing term is used within this research to drive the adjoint sensitivity analysis. The results demonstrate the influence of source-specific emission reductions on domainwide mortality from chronic exposure to $\mathrm{O}_{3}, \mathrm{NO}_{2}$, and $\mathrm{PM}_{2.5}$.

The above equation 3.14 describes the relationship between pollutant exposure and monetized human health. This exposure-health relationship can be directly applied to CTM concentration outputs to estimate mortality due to exposure, opposed to MB (mortality change due to emission reduction). It is used within this thesis as both a direct method of estimating pollution exposure related mortality at predicted concentrations and to estimate MB. 


\subsection{Impacts of grid resolution on adjoint-based marginal benefit estimates of air pollution exposure}

This chapter is in the format of a draft manuscript that is in preparation for publication in Air Quality, Atmosphere, and Health, or a similar journal. It contains original research with Melanie Fillingham as the main contributor $(80 \%)^{1}$.

\section{Abstract}

The monetized societal benefit of emission reductions is known as marginal benefit (MB), which can be estimated for air pollutant emissions by integrating air quality models with and epidemiological models. This method is subject to uncertainties arising from model grid resolution. Using the adjoint of gas-phase CMAQv5.0.2, this study analyzes the impact of grid resolution on MBs, parametrized as changes to mortality from chronic exposure to ozone, $\mathrm{O}_{3}$, and nitrogen dioxide, $\mathrm{NO}_{2}$, due to emission reductions of nitrogen oxides, NOx. Furthermore, the impacts of resolution on concentration predictions of $\mathrm{O}_{3}, \mathrm{NO}_{2}$ and $\mathrm{PM}_{2.5}$ were evaluated. Grid resolution impacts were evaluated by modelling at progressively refined resolutions of $36,12,4$ and $1 \mathrm{~km}$, while taking $1 \mathrm{~km}$ as the benchmark.

\footnotetext{
${ }^{1}$ Co-authors on this work include: Burak Oztaner, Shunliu Zhao, Amir Hakami (Carleton University); Amanda Pappin (Health Canada); Wayne Boulton, Greg Conley, Martin Gauthier, Jeff Lundgren, Julia Veerman,Carol McClellen(RWDI Inc.); Louise Aubinm, Eric Tran, Kim McAdam (Regional Municipality of Peel Public Health Department); and the CMAQ Adjoint Development Team
} 
The results indicate that both coarse modeled maxima MBs and concentrations are underestimated in urban areas and overestimated in rural areas that are nearby urban cores. The maximum July MB results at $1 \mathrm{~km}$ resolution was $\$ 618,000 /$ tonne NOx, whereas at $36 \mathrm{~km}$ resolution was $\$ 359,000 /$ tonne NOx, demonstrating the dilution of maxima at coarse resolution. The domain-wide aggregated monthly total damages $(\$$ /month) were predicted and compared between resolutions, in which there was not a high discrepancy, suggesting that if interested in total health impacts, there may not substantial benefit to reducing to a $1 \mathrm{~km}$ resolution. Coarse modelling results did not pick up localized NOx inhibitions (which cause negative MBs), and as such, aggregated domain-wide damages were highest at $36 \mathrm{~km}$ for July (\$310 million) and lowest at $1 \mathrm{~km}$ (\$106 million). Concentration results were dependent on the species, having the highest impact due to resolution on $\mathrm{PM}_{2.5}$, followed by $\mathrm{NO}_{2}$, and the least impacts on $\mathrm{O}_{3}$. Additionally, fine resolution results show a higher level of spatial detail allowing for depiction of specific locations in which highest benefits are seen, highlighting locations in which emission reduction should be focused.

Key Words: marginal benefit, adjoint sensitivity, grid resolution, air quality health impacts

\subsection{Introduction}

Air quality poses a significant challenge in our society due to its direct impact on human health and mortality, resulting in substantial social and economic burdens. Ground-level ozone $\left(\mathrm{O}_{3}\right)$, nitrogen dioxide $\left(\mathrm{NO}_{2}\right)$, and fine particulate matter $\left(\mathrm{PM}_{2.5}\right.$, particulate matter 
with an aerodynamic diameter $<2.5 \mu \mathrm{m}$ ) are pollutants that have shown association with adverse human health effects in Canada, especially related to cardiovascular and respiratory illnesses (Anenberg et al., 2010; Krewski et al., 2009; Pope et al., 2002). Research has proven both short-term and long-term negative health effects due to exposure to these pollutants (Burnett et al., 2004; Jerrett et al., 2009; Chen \& Kan, 2008; Pope et al., 2002). In Canada, using the Canadian Census Health and Environment Cohort (CanCHEC), Crouse et al. (2015) found that long-term exposure to $\mathrm{O}_{3}$ increased the risk of non-accidental mortality by $0.188 \% / p p b(D M 8 A)$, and that long-term exposure to $\mathrm{NO}_{2}$ increased the risk by $0.129 \% / p p b$ (daily average). Another study using the CanCHEC cohort found that long-term exposure to $\mathrm{PM}_{2.5}$ increased non-accidental mortality by $1.6 \%$ per $\mu \mathrm{g} / \mathrm{m}^{3}$ (Pinault et al., 2017).

The effects of air pollution on human health can be evaluated by benefit-cost analysis techniques that involve the use of computational air quality models (AQMs) paired with epidemiological data/models. Marginal benefits (MBs), which represent monetized health or environmental benefit due to a reduction in a unit (e.g. 1 tonne) of emissions, is one method of quantifying health impacts of anthropogenic emissions. Adjoint sensitivity modeling of chemical transport models (CTMs) has been paired with epidemiological data to successfully analyze MB at coarse (e.g. $36 \mathrm{~km}$ ) resolutions (Pappin \& Hakami, 2013a, 2013b; Pappin et al., 2015, 2016). Adjoint sensitivity analysis is a backward sensitivity method that starts with the receptor (receptor-based) and illustrates where the influences are sourced from, giving representation of the impact of 
all emission sources on a specified domain. The accuracy of these predictions is dependent on the model grid resolution used. Detailed explanation of adjoint sensitivity analysis is beyond the scope of this work and can be found elsewhere (Sandu et al., 2005; Hakami et al., 2007; Henze et al., 2007)

There is extensive research analyzing the impacts of model resolution on CTM concentration predictions for $\mathrm{O}_{3}$ (Arunachalam et al., 2006; Cohan et al., 2006; Wild \& Prather, 2006; Queen \& Zhang, 2008; Valari \& Menut, 2009; Tie et al., 2010; Hodnebrog et al., 2011), for $\mathrm{NO}_{2}$ (Vautard et al., 2009; Valin et al., 2011; Schaap et al., 2015; Tan et al., 2015; Kuik et al., 2016), and for $\mathrm{PM}_{2.5}$ (Arunachalam et al., 2011; Stroud et al., 2011; ; Fountoukis et al., 2013; Punger \& West, 2013; Schapp et al., 2015). Numerous processes within CTMs are distinctively affected by resolution, such as numerical discretization, emission processing, transport (meteorology and topography), and chemistry. Inaccuracies can arise as coarse resolutions do not adequately represent the spatial variability of model parameters as averaging occurs, causing dilution within each grid (Tie et al., 2010; Punger \& West, 2013). Dependence on grid resolution is especially significant in urban/suburban areas where the spatial concentration gradients are strong (Thompson et al., 2014). Coarse grid resolutions often result in underestimated pollutant concentrations in urban areas due to the artificial dilution of emissions (Tie et al., 2010; Punger \& West, 2013), while in contrast, they lead to overestimated pollution concentrations in rural areas (Arunachalam et al., 2006) when compared to monitored 
data. The dilution of $\mathrm{O}_{3}$ precursor species alters $\mathrm{O}_{3}$ titration processes and formation chemistry.

A pollutant's lifetime may influence its susceptibility to grid resolution impacts. Longlived pollutants often have a more homogeneous spatial distributions due to sufficient time for transport and mixing compared to short-lived pollutants which have high localized concentrations near their source. It has been suggested that grid resolution does not influence long-lived species as significantly as those that are short-lived (US, EPA. 2007; Punger \& West, 2013). Tropospheric $\mathrm{O}_{3}$ is a relatively long-lived species with an average atmospheric lifetime in the northern hemisphere of 23 days, $\mathrm{NO}_{2}$ has a short tropospheric lifetime ranging from a couple of hours to days, depending on environmental conditions, while $\mathrm{PM}_{2.5}$ is composed of many different species, with atmospheric lifetimes that range from days to weeks (Seinfeld \& Pandis, 2006). Research has demonstrated the response to grid resolution be strongest for $\mathrm{NO}_{2}$, followed by PM, and weakest for $\mathrm{O}_{3}$ (Queen \& Zhang, 2008; Schaap et al., 2015).

Punger and West (2013) assessed mortality due to $\mathrm{O}_{3}$ exposure at resolutions between 12 and $408 \mathrm{~km}$ and found that there was only a maximum of $6 \%$ difference between the coarse and fine resolutions. In this same study, Punger and West (2013) also estimated total U.S.-wide mortality associated with $\mathrm{O}_{3}$ exposure and found that $36 \mathrm{~km}$ resolution estimates were $12 \%$ higher than those generated from $12 \mathrm{~km}$ resolution, suggesting a potential overestimation at coarse resolutions. Thompson and Selin (2012) found a 
similar conclusion where domain-wide total health impacts due to $\mathrm{O}_{3}$ exposure were overestimated at $36 \mathrm{~km}$, compared to those generated at 12, 4 or $1 \mathrm{~km}$ resolutions. It is difficult to assess the accuracy of health impacts, and as such an over or underestimate relative to another resolution may not necessarily be causing a less accurate prediction. There was a lack of studies related to resolution and $\mathrm{NO}_{2}$ related health impacts found throughout this review, however, studies analyzing resolution impacts on $\mathrm{PM}_{2.5}$ related health impacts had different conclusions than for $\mathrm{O}_{3}$, indicating that the influence of model resolution be reliant on species. For example, Arunachalam et al. (2011) found that aggregated total health risks due to $\mathrm{PM}_{2.5}$ exposure not change drastically between resolutions of 36,12 and $4 \mathrm{~km}$.

While there are numerous studies assessing the impact of resolution on modelled concentrations, there are fewer studies that have been conducted linking direct health impact results from pollutant concentrations to resolution (Arunachalam et al., 2011; Thompson \& Selin, 2012; Punger \& West, 2013; Thompson et al., 2014; Li et al., 2015), and no research has studied the resolution impacts on $M B$ estimations. $M B$ is a function of both concentration and population, two factors which are susceptible to the impacts of grid resolutions, indicating that MB will also be influenced by resolution.

The adjoint approach allows for the spatiotemporal influences of specific emission sources to be traced and quantified. When using high resolution models, specific emission sources can be more accurately determined. Influences of individual/specific 
emission sources are more difficult to isolate with coarse resolution models as there may be multiple sources within a single coarse grid cell. Contributions of individual sources on health impacts provide beneficial information for formulating emission control policies. Previous research attributing the effects of air pollution to specific emission sources has found that health benefits associated with emission reduction were significant but highly dependent on the location of emission (Pappin \& Hakami, 2013). Source-specific benefit attributions provide decision makers with a direct measure of return of investments for policies that are aimed at improving air quality at large.

In this study, the impacts of grid resolution on modelled concentrations and health impacts were evaluated through modelling both concentration and MBs at progressively refined resolutions of $36,12,4$, and $1 \mathrm{~km}$, with the domain set over the western part of Lake Ontario. The analysis of resolution impacts on concentration was performed for $\mathrm{O}_{3}$, $\mathrm{NO}_{2}$, and $\mathrm{PM}_{2.5}$, while for health impacts $(\mathrm{MB})$ was performed for $\mathrm{O}_{3}$ and $\mathrm{NO}_{2} . \mathrm{MBs}$ were not estimated for $\mathrm{PM}_{2.5}$ as at the time of the study, the adjoint of aerosols was still under development. $\mathrm{MB}$ in this research is parametrized as the change to mortality from chronic exposure to $\mathrm{O}_{3}$, and $\mathrm{NO}_{2}$, combined, due to emission reductions of nitrogen oxides, $\mathrm{NOx}$ (a combination of $\mathrm{NO}_{2}$ and nitric oxide, $\mathrm{NO}$ ). MBs were predicted by integrating epidemiological data with the adjoint of the Community Multi-scale Air Quality Model, CMAQ. 


\subsection{Methodology}

\subsubsection{Adjoint Sensitivity Analysis}

Sensitivity modeling demonstrates how model outputs change with respect to model inputs; in the case of CTMs, how concentration predictions change with respect to emission inputs. Adjoint sensitivity is the backward sensitivity approach used to conduct this study, for details on the adjoint model explanation, refer elsewhere (Hakami et al., 2007; Henze et al., 2007; Sandu et al., 2005). To obtain sensitivity in the sense of MB (defined in this paper as the monetary societal benefit (\$) of a 1 tonne reduction of $\mathrm{NO}_{x}$ emissions), a relationship between epidemiological models and adjoint sensitivity can be used, which is applied through what is known as the adjoint cost function. In this research, the cost function $(\mathrm{M})$ represents the monetary value of mortality in a specified domain with respect to exposure to the pollutant of interest $\left(\mathrm{O}_{3}\right.$ and $\left.\mathrm{NO}_{2}\right)$. The change in mortality valuation $(\Delta \mathrm{M})$ resulting from a change in pollutant concentration $(\Delta \mathrm{C})$ mortality can be expressed by:

$$
\Delta M=M_{0} \times P \times V_{S L}\left(1-e^{\beta \Delta C}\right)
$$

Where $M_{0}$ is the baseline nonaccidental mortality rate, $P$ is the population, $V_{S L}$ is the value of statistical life, and $\beta$ is the concentration response function (CRF).

The change in the cost function with respect to the change in concentration is known as the adjoint forcing term and is determined from the differentiation of equation 4.1, shown as follows in equation 4.2:

$$
\phi=\frac{\partial M}{\delta C}=\frac{V_{S L} M_{0} P \beta e^{-\beta C}}{t n}
$$


Where $\phi$ is the adjoint forcing term, $t$ is the hours in the exposure metric (i.e. 8 hours for $\mathrm{O}_{3}$ as the daily maximum 8-hour average, $\mathrm{DM} 8 \mathrm{~A}$, is the $\mathrm{O}_{3}$ exposure metric; 24 for $\mathrm{NO}_{2}$ as daily average is the $\mathrm{NO}_{2}$ exposure metric), $\mathrm{n}$ is the number of days in the period of simulation (365 days in this case as all results are expressed as "annually"), and the remaining variables on the right-side have the same definitions as in equation 4.1.

The forcing term applied within this research was for the long-term exposure related mortality due to the combination of the average 24-hour (daily) $\mathrm{NO}_{2}$ exposure and the DM8A $\mathrm{O}_{3}$ exposure. The combination of the $\mathrm{O}_{3}$ and $\mathrm{NO}_{2}$ forcing terms gives accumulated results with respect to both $\mathrm{O}_{3}$ and $\mathrm{NO}_{2}$ exposure. The forcing term was applied only to the area covered by the $1 \mathrm{~km}$ domain for all resolutions, as this is the common area between all domains in which comparisons are made (refer to Section 4.2.3). In this research, the adjoint sensitivity portrays the influence of $\mathrm{NO}_{\mathrm{x}}$ emissions at specific source locations on mortality due chronic exposure to $\mathrm{O}_{3}$ and $\mathrm{NO}_{2}$ (combined); the results show domain-wide $\mathrm{MB}$ due to a reduction in $\mathrm{NO}_{\mathrm{x}}$ emissions.

\subsubsection{Health Valuation Parameters}

The Canadian VSL was produced from statistical information gathered within Canada through the Policy Research Initiative, with the most recent valuing at $\$ 7.17 \mathrm{M}$ (Chestnut et al., 2009). The CRFs for chronic exposure to $\mathrm{O}_{3}$ and $\mathrm{NO}_{2}$ used values of $0.27 \%$ change in mortality (non-accidental) /ppb change (DM8A) and $0.59 \%$ change in mortality (nonaccidental) /ppb change (daily average), respectively (Crouse et al., 2015). The CRF is 
applicable to population aged twenty-five and older. Population data was obtained from Statistics Canada (Census Dissemination Area) from the 2011 Census and included only the population aged twenty-five plus to match the CRF demographics. The required Canadian baseline mortality rates (BMR, non-accidental mortality, aged twenty-five plus) were obtained from Health Canada's Air Quality Benefits Assessment Tool (AQBAT) (Judek et al., 2006) for each Census Division. The BMR data was population weighted and then gridded to the respective resolution $(1,4,12$, and $36 \mathrm{~km})$ using the Community Modeling and Analysis System's (CMAS) spatial allocator tool. Population was also aggregated to the respected resolutions using spatial allocator.

\subsubsection{Case Study Model Parameters}

CMAQv5.0.2-adjoint was run at progressively refined horizontal resolutions $(36,12,4$, and $1 \mathrm{~km}$ resolution) over the four domains shown in Figure 4-1, with the $36 \mathrm{~km}$ resolution as the parent domain, and 12,4 and $1 \mathrm{~km}$ resolutions for the respective nested domains within. The vertical resolution remained consistent. The temporal resolution (timestep) was 12 minutes for the 36 and $12 \mathrm{~km}$ resolutions, 5 minutes for the $4 \mathrm{~km}$ resolution, and 1.5 minutes for the $1 \mathrm{~km}$ resolution. 


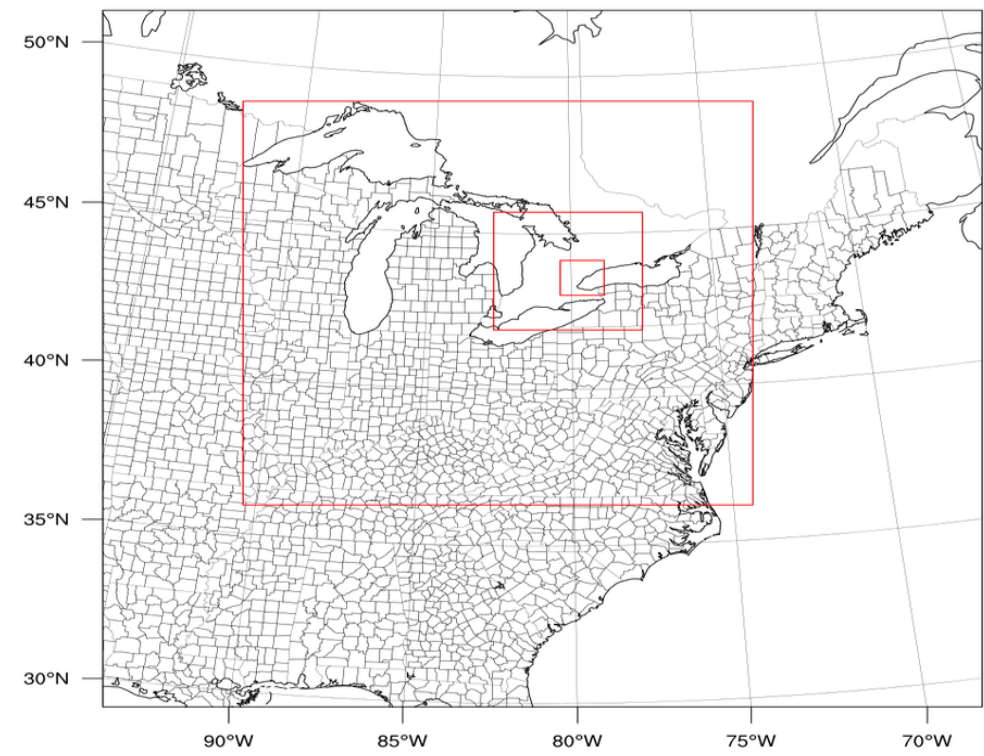

Figure 4-1. Nested domain configuration, resolutions are 36 (main domain), 12, 4 and 1 km (inner-most domain), respectively, focusing in on western Lake Ontario.

The simulation period was over the first two weeks of both February and July, 2012, averaging and extrapolating the results to represent annual MBs. The inner-most domain covered the western part of Lake Ontario, which includes the Greater Toronto Hamilton Area (GTHA). The U.S. emissions were sourced from the 2008 National Emission Inventory (NEI). The Canadian emissions were sourced from Environment and Climate Change Canada's (ECCC) 2010 National Pollution Release Inventory (NPRI) for point sources, the 2011 National Agri-Environmental Standards Initiative for area sources, and the remaining area and mobile emissions from ECCC's 2006 Criteria Air Contaminants (CACs). The emissions for the common areas of each successive resolution were aggregated from the fine data. Meteorological modelling was carried out for each resolution (nested) using the Weather Research and Forecast (WRF) model version 3.4.1 (Skamarock et al., 2008). The meteorological data was processed for each domain at the respective resolution. 
The adjoint cost functions were applied only to the inner-most domain $(1 \mathrm{~km})$, masking outside areas. This indicates that the adjoint cost function is defined as the monetized societal cost in GTHA, and that the results show only the marginal benefit seen from receptors within the $1 \mathrm{~km}$ domain, allowing for direct comparisons between the different resolutions. Boundary conditions from the subsequent resolution (e.g. $12 \mathrm{~km}$ boundaries were generated from $36 \mathrm{~km}$ results) were incorporated, allowing for the impacts of pollution that may have traveled outside and returned to the domain to be accounted for.

\subsection{Results and Discussion}

\subsubsection{Concentrations: Progressively Refined Resolutions}

Modelled July surface concentration results for $\mathrm{O}_{3}, \mathrm{NO}_{2}$ and $\mathrm{PM}_{2.5}$ were averaged based off their respective standard averaging period (maximum daily 8-hour average for $\mathrm{O}_{3}$, 24-hour daily average for $\mathrm{NO}_{2}$ and $\mathrm{PM}_{2.5}$ ) and then averaged over each of the 2-week periods in the modelled months (first 2 weeks in July/February). The concentration results for July and February are displayed in Figures 4-2 and 4-3, respectively. As expected, the fine resolution model results show more spatial detail and better depict spatial gradients, both of which become progressively worse as the resolution becomes coarser. It can be seen from just observing the Figures (4-2 and 4-3) that in both July and February, $\mathrm{O}_{3}$ is the most spatially homogenous (geographically uniform) out of the species analyzed while $\mathrm{PM}_{2.5}$ and $\mathrm{NO}_{2}$ are more spatially heterogeneous with the 
strongest spatial gradients, a result of their respective atmospheric lifetimes.

Tropospheric $\mathrm{O}_{3}$ is a relatively long-lived species with an average atmospheric lifetime in the northern hemisphere of 23 days, while $\mathrm{NO}_{2}$ has a short lifetime ranging from a couple of hours to days, $\mathrm{PM}_{2.5}$ is composed of many different species, with atmospheric lifetimes that range from days to weeks (Seinfeld \& Pandis, 2006). The influence grid resolution has on each species is correlated to their spatial homogeneity, a function of their atmospheric lifetime and emission source distribution. Ozone was the least impacted by grid resolution, while $\mathrm{NO}_{2}$ and $\mathrm{PM}_{2.5}$ were both highly impacted, results consistent with other studies (U.S. EPA, 2007; Punger \& West, 2013). 

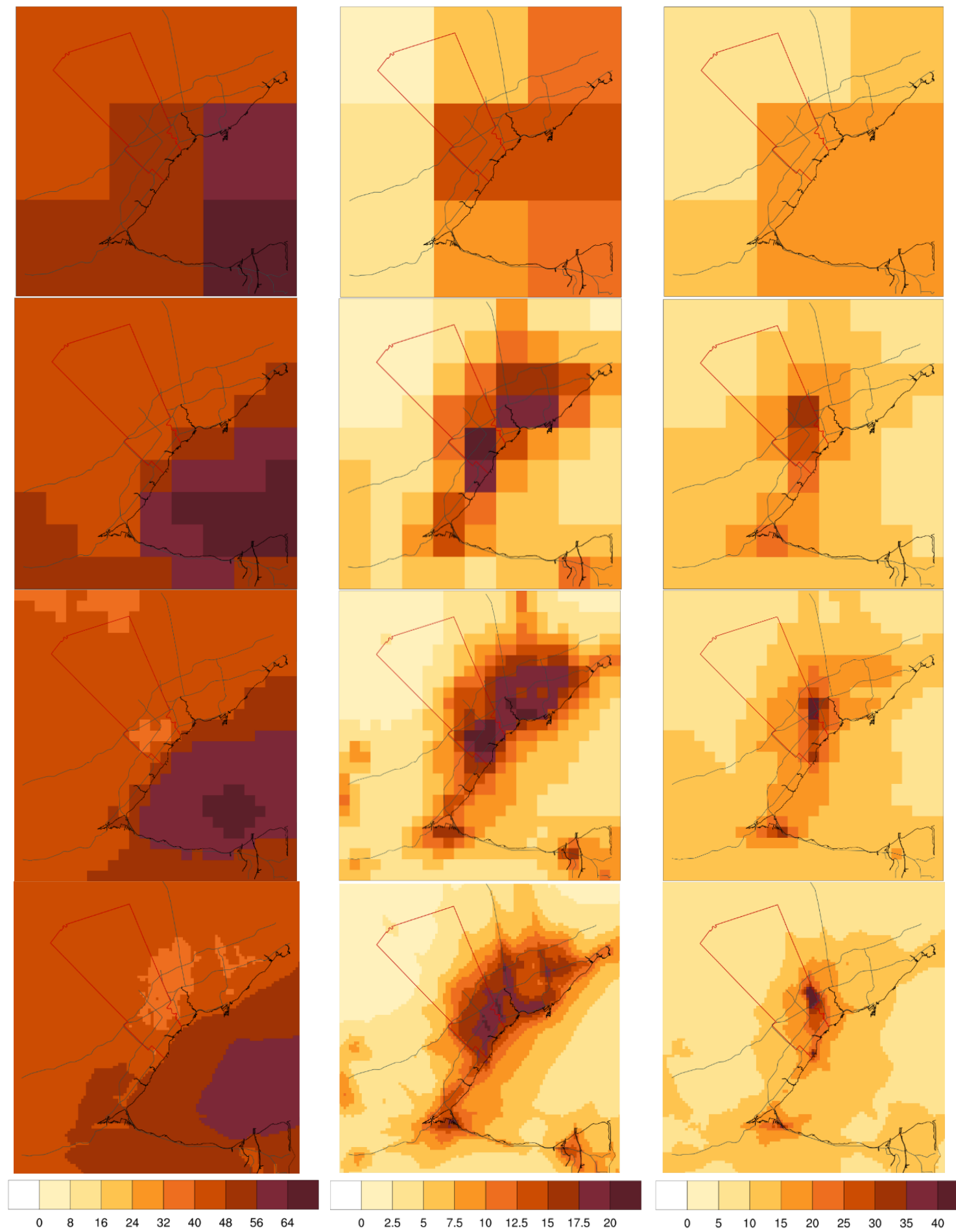

$\mathrm{ppb}$
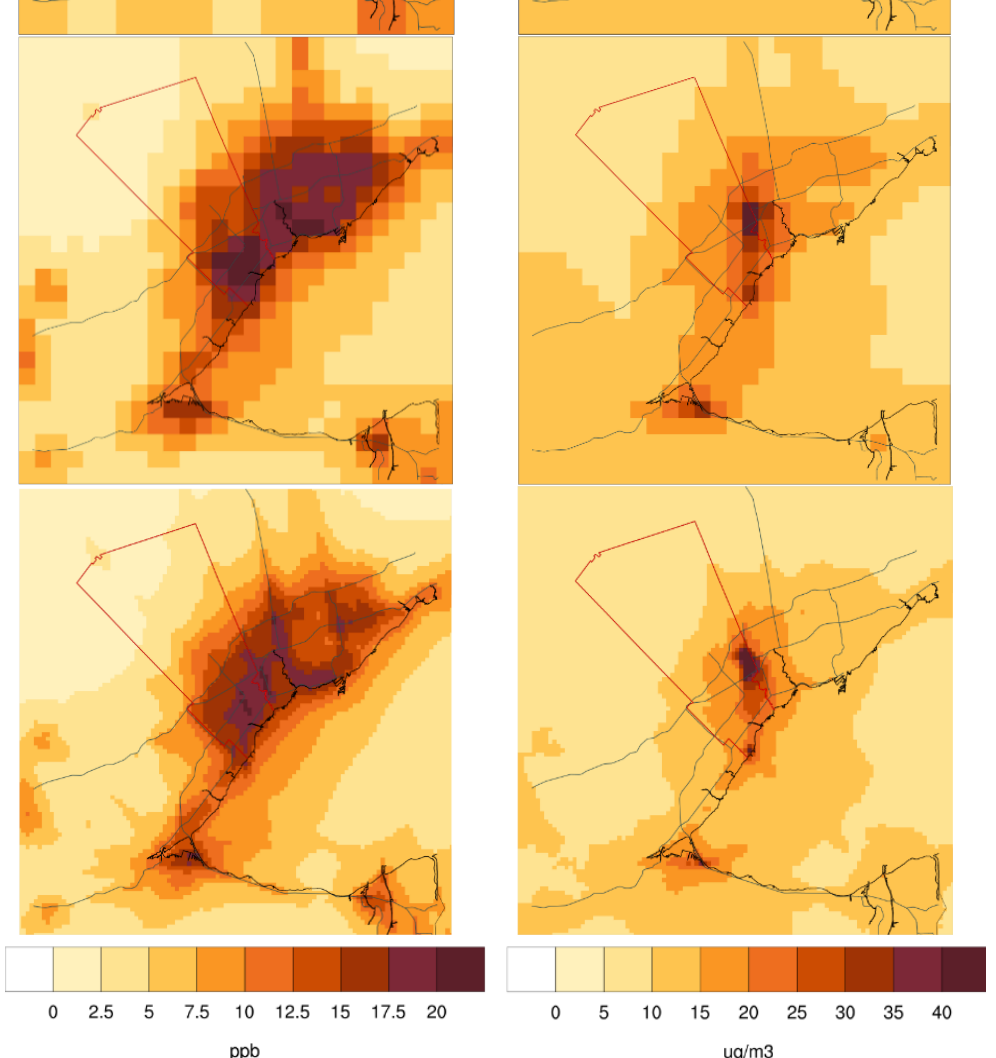

Figure 4-2. Concentration results for $36,12,4$, and $1 \mathrm{~km}$ (top to bottom) for $\mathrm{O}_{3}, \mathrm{NO}_{2}$, and $\mathrm{PM}_{2.5}$ (left to right), averaged to their respective standard averaging period and over the July 2 week period. 

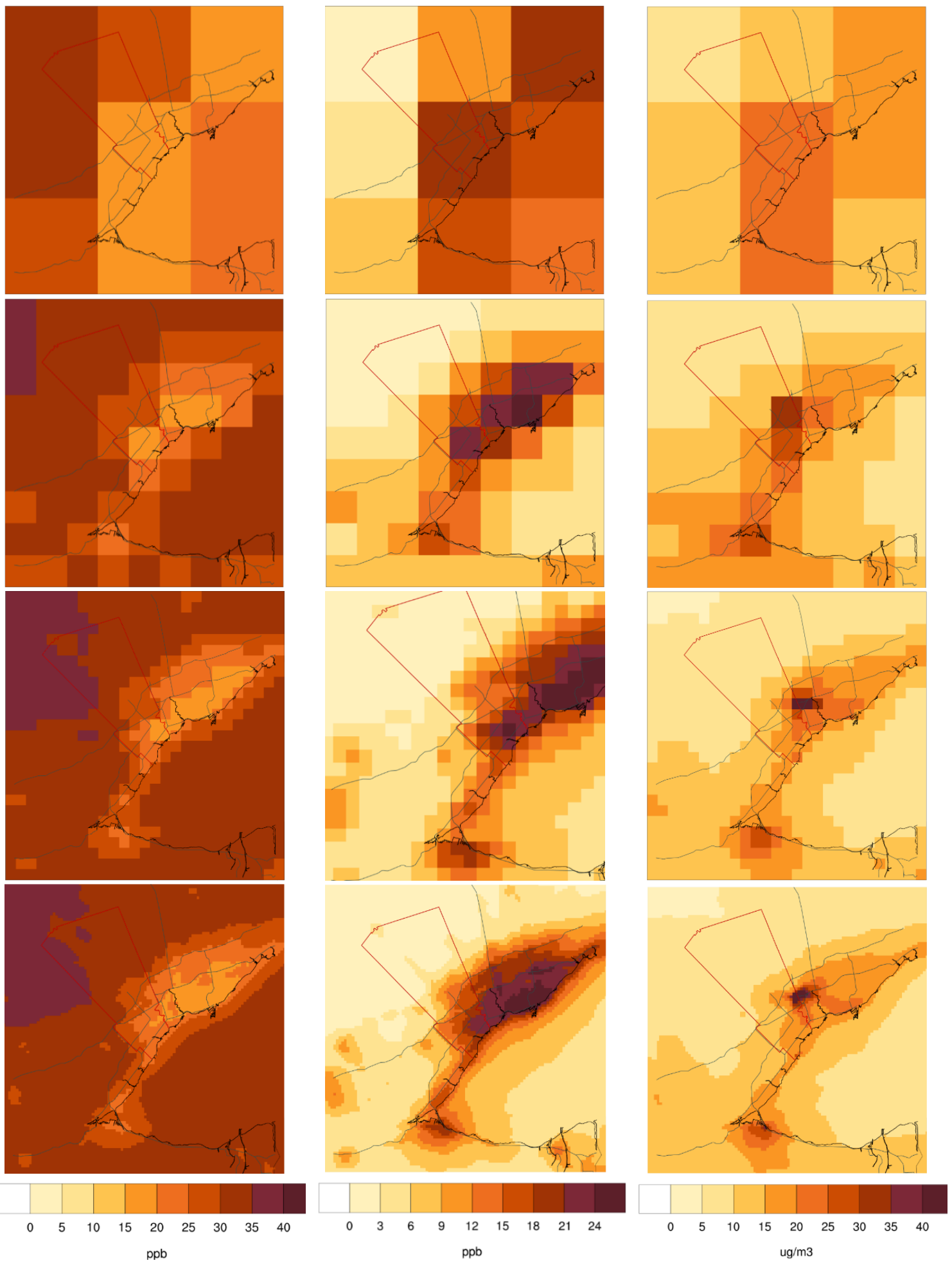

Figure 4-3. Concentration results for $36,12,4$, and $1 \mathrm{~km}$ (top to bottom) for $\mathrm{O}_{3}, \mathrm{NO}_{2}$, and $\mathrm{PM}_{2.5}$ (left to right), averaged to their respective standard averaging period and over the February 2 week periods. 
It was expected that the maximum concentrations diminish as the resolution became coarser due to dilution within each grid cell, however, this was not necessarily the case. The decrease in maximum concentrations trend was seen for all species in February but only for $\mathrm{PM}_{2.5}$ in July (Table 4-1). In July, $\mathrm{O}_{3}$ concentration increased as the resolution became coarser, while $\mathrm{NO}_{2}$ increased from $1 \mathrm{~km}$ until $12 \mathrm{~km}$ and then decreased at $36 \mathrm{~km}$. The maximum $\mathrm{PM}_{2.5}$ concentration always decreased as the resolution became coarser, regardless of the month.

Referring to Table 4-1, at $36 \mathrm{~km}$ resolution, the maximum $\mathrm{NO}_{2}$ concentrations were substantially lower than those from the finer resolutions for both July and February. The highest direct $\mathrm{NO}_{2}$ maximum concentration change (between two subsequent resolutions) occurred between 12 and $36 \mathrm{~km}$, dropping from $23.1 \mathrm{ppb}$ to $14.9 \mathrm{ppb}$. The maximum $\mathrm{NO}_{2}$ concentrations occurred within the urban areas surrounding Toronto, corresponding with locations of high emission sources. $\mathrm{NO}_{2}$ concentrations generated at $36 \mathrm{~km}$ were greatly underestimated in urban areas, a result of averaging within each grid, which therefore overestimated concentrations in the rural/suburban areas within close proximity.

Maximum $\mathrm{PM}_{2.5}$ concentrations were highly influenced by grid resolution, seeing large discrepancies even between the fine resolutions; the $1 \mathrm{~km}$ maximum 24-hour concentrations (Table 4-1) were over double those of the $4 \mathrm{~km}$ maximums in both July and February. As the resolution became coarser, the maximum $\mathrm{PM}_{2.5}$ concentration continues to substantially decrease within both months. These large deviations between maximum concentrations at 
different resolutions is a result of emission dilution at the coarse resolution. The location in which the maximum $\mathrm{PM}_{2.5}$ concentration occurs is the location of the Toronto Pearson airport, which is a localized source of high $\mathrm{PM}_{2.5}$ emissions. The short-lived $\mathrm{PM}_{2.5}$ components have short dispersal ranges and high concentrations close to emission sources, which coarse resolutions are unable to characterize. The extreme heterogeneity of concentrations seen in Figure 4-2 and 4-3 is influenced by strong localized emission sources, which are not as spatially uniform as those of $\mathrm{NO}_{2}$. The large discrepancies near emission sources suggest that they are most likely a result of resolution impacts on all primary $\mathrm{PM}_{2.5}$ species. Ott et al. (2008) demonstrated that PM concentrations were heterogeneous over short distances by monitoring PM at a series of stations with an average of $4.4 \mathrm{~km}$ between sites. The large concentration gradients within these results over short distances agree with results found by Ott et al.

Modelled $\mathrm{O}_{3}$ concentrations show the least influence due to grid resolution (Table 4-1), relative to $\mathrm{NO}_{2}$ or $\mathrm{PM}_{2.5}$, as it is more homogeneous over the domain. Fine particulate matter concentrations had the highest influence due to grid resolution. These findings are consistent with other studies (Queen \& Zhang, 2008; Schaap et al., 2015).

Table 4-1. Maximum modelled concentrations of $\mathrm{O}_{3}, \mathrm{NO}_{2}$ and $\mathrm{PM}_{2.5}$ at each resolution (1, 4, 12 and $\left.36 \mathrm{~km}\right)$ for the July and February results

Max. Concentration*

\begin{tabular}{cccccc} 
& & $\mathbf{1} \mathbf{~ k m}$ & $\mathbf{4 ~} \mathbf{~ m}$ & $\mathbf{1 2} \mathbf{~ k m}$ & $\mathbf{3 6} \mathbf{~ k m}$ \\
\hline \multirow{2}{*}{$\mathbf{O}_{\mathbf{3}}(\mathbf{p p b})$} & July & 60.8 & 65.5 & 68.2 & 67.6 \\
& February & 36.0 & 35.8 & 35.2 & 32.4 \\
\hline \multirow{2}{*}{$\mathbf{N O}_{\mathbf{2}}(\mathbf{p p b})$} & July & 20.6 & 23.5 & 23.1 & 14.9 \\
& February & 29.6 & 28.4 & 24.8 & 19.0 \\
\hline
\end{tabular}




\begin{tabular}{cccccc}
\hline $\mathbf{P M}_{2.5}\left(\boldsymbol{\mu g} / \mathrm{m}^{3}\right)$ & July & 164.8 & 67.6 & 32.5 & 17.9 \\
& February & 138.7 & 66.6 & 24.8 & 20.8 \\
\hline
\end{tabular}

*Maximum concentration of the mean week-long averaged concentration based off the respective standard averaging period ( $\mathrm{DM} 8 \mathrm{H}$ for $\mathrm{O}_{3}, 24$-hour average for $\mathrm{NO}_{2}$ and $\mathrm{PM}_{2.5}$ )

Concentration results from each relatively fine resolution were aggregated up to each relatively coarse resolution for all model resolution combinations (i.e. 1 to $36 \mathrm{~km}, 1$ to $12 \mathrm{~km}$ and 1 to 4 $\mathrm{km}, 4$ to $12 \mathrm{~km}$ and 4 to $36 \mathrm{~km}$, and 12 to $36 \mathrm{~km}$ ), for each species over their respective standard averaging period. These results are displayed in Figures 4-4, 4-5, and 4-6 for $\mathrm{O}_{3}, \mathrm{NO}_{2}$, and $\mathrm{PM}_{2.5}$, respectively. These figures emphasize a gradual shift in concentration patterns going from fine to coarse resolutions. The resolution-induced misrepresentation seems to become more pronounced as the resolution becomes coarser. The coarse resolution generated results for all three of the analyzed species show areas in which the concentrations were higher than when the fine resolution results were aggregated up, with the largest difference between 1 and $36 \mathrm{~km}$, and the least difference between 12 and $36 \mathrm{~km}$. These results show a trend of coarser resolutions over-estimating total pollutant mass over a domain, despite consistency in domain wide emissions. 


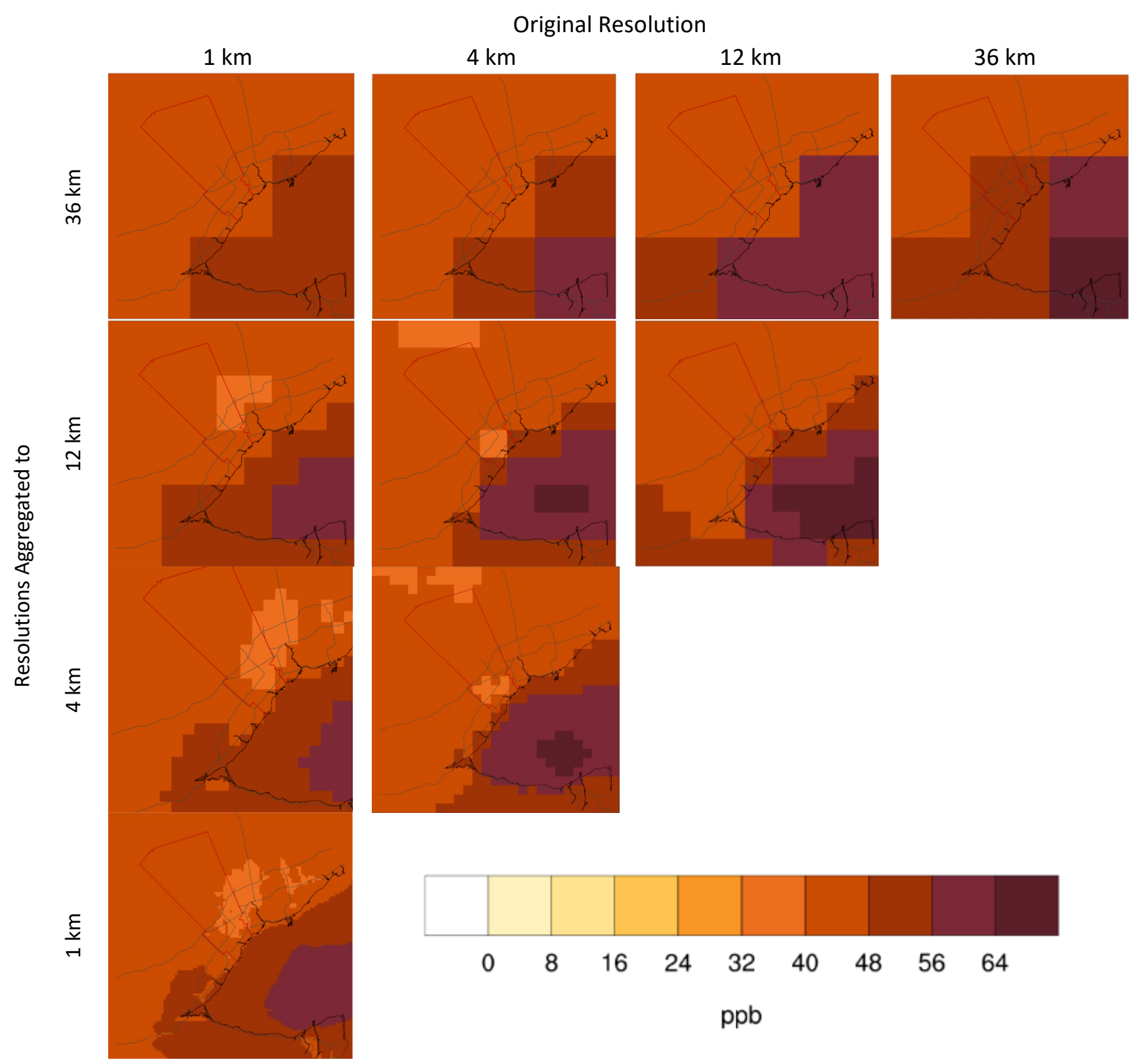

Figure 4-4. Matrix of July first 2-weeks average $\mathrm{DM} 8 \mathrm{~A} \mathrm{O}_{3}$ concentrations aggregated to each separate coarse resolution. 


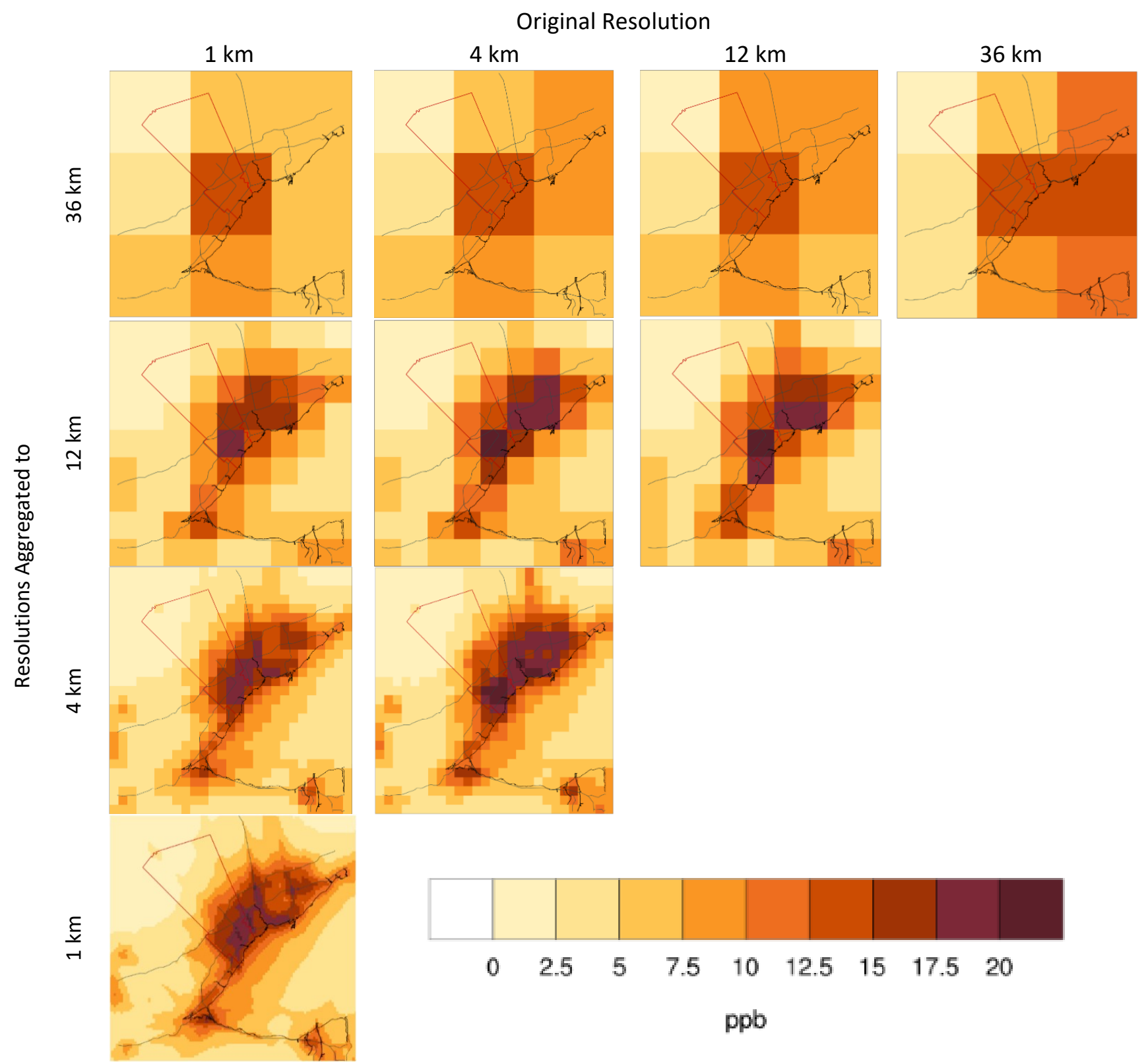

Figure 4-5. Matrix of July first 2-weeks average 24-hour $\mathrm{NO}_{2}$ concentrations aggregated to each separate coarse resolution. 


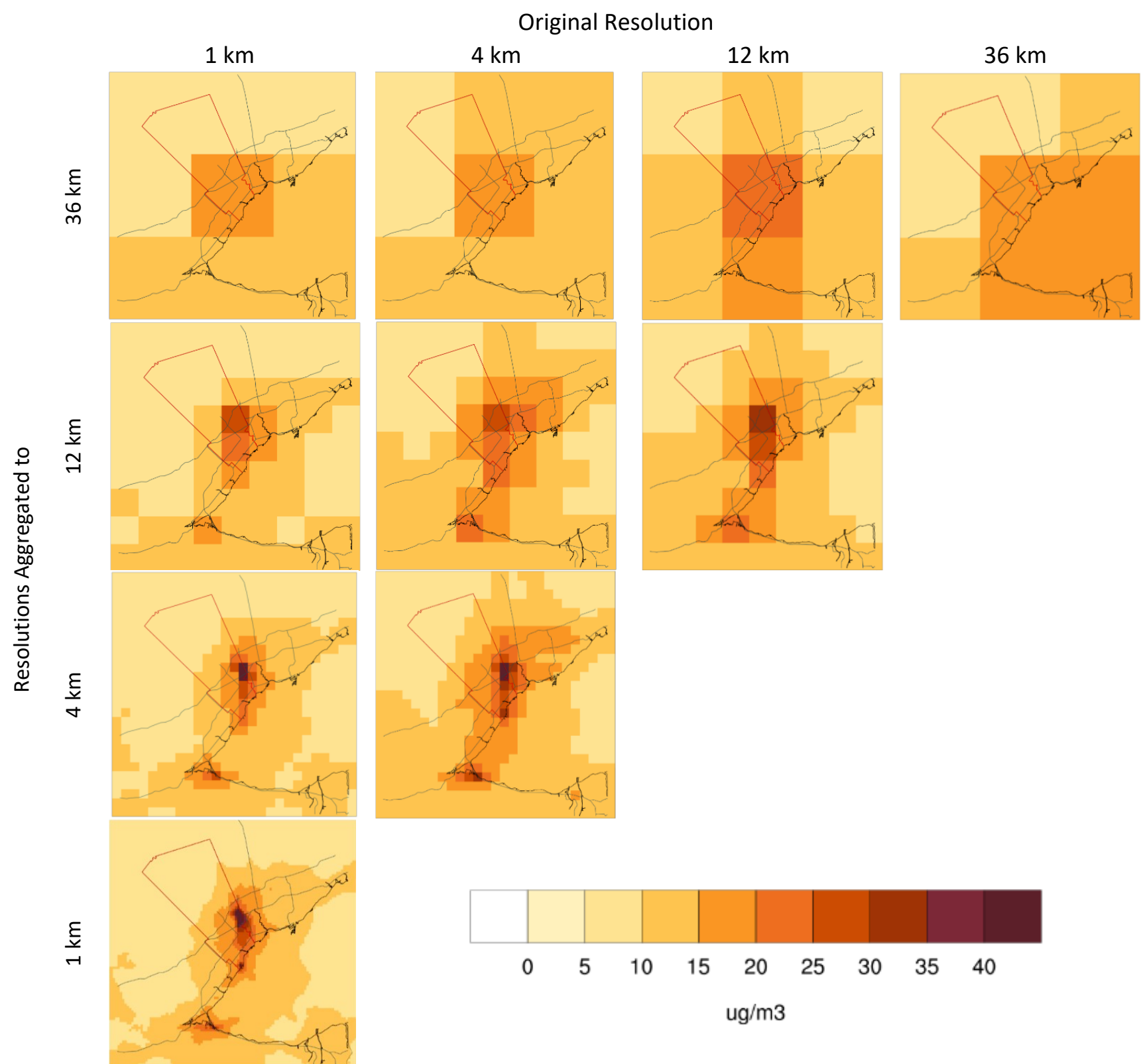

Figure 4-6. Matrix of July first 2-weeks average 24-hour $\mathrm{PM}_{2.5}$ concentrations aggregated to each separate coarse resolution.

\subsubsection{Marginal Benefits: Progressively Refined Resolutions}

The adjoint cost functions for various resolutions, as previously defined, were set up to find the combined monetized long-term $\mathrm{O}_{3}$ and $\mathrm{NO}_{2}$ exposure related mortality with respect to change in anthropogenic $\mathrm{NO}_{x}$ over the common domain occupied by the $1 \mathrm{~km}$ resolution area. Both $\mathrm{O}_{3}$ 
and $\mathrm{NO}_{2}$ forcing were combined such that the results depict the accumulated sensitivity to the two species. Emission weighted (across time) model results for both July and February are displayed in Figure 4-7. The results depict how a change of 1 tonne of $\mathrm{NO}_{\mathrm{x}}$ emissions in the specific location (i.e. individual grid cells) will impact the monetized long-term mortality (due to exposure to $\mathrm{O}_{3}$ and $\mathrm{NO}_{2}$ ) over the domain in which the forcing term was applied (for each case, the area covered by the $1 \mathrm{~km}$ resolution). This is likely to result in underestimated MBs (especially for $\mathrm{O}_{3}$, a longer-lived species than $\mathrm{NO}_{2}$ ) as health impacts occurring outside of the 1 $\mathrm{km}$ domain caused by long-range travel of pollution sourcing from within the domain are not considered. Since boundary conditions were generated from the subsequent coarser resolution, the impacts of $\mathrm{O}_{3} / \mathrm{NO}_{2}$ that has transported outside of, and returned to the domain are accounted for.

As expected, the MB results (Figure 4-7) for both months show more spatial detail at finer resolutions. Since MB results are source-specific, the influence from individual emission sources can be more accurately determined at fine resolution models. This is because within a $1 \mathrm{~km}$ grid cell ( $1 \mathrm{~km} \times 1 \mathrm{~km}$ area) there are fewer emission sources compared to the multiple that may be present in a $12 \mathrm{~km} \times 12 \mathrm{~km}$ or $36 \times 36$ area. The impacts of air pollution can be attributed to specific sources when utilizing fine resolution modelling, which is substantial for air quality management which relies on emission source control.

The February results are less homogenous than those seen in July, with majority of the high MB results localized around the urban area. The July results also have localized high MB in locations 
of high population but are still relatively high outside of the urban area, differing from those seen in February. This difference may be attributed to the seasonal influences on the production and atmospheric lifetimes of $\mathrm{O}_{3}$ and $\mathrm{NO}_{2}$. Ozone, a photochemically produced compound, is produced at higher rates (hence higher concentrations, as validated by the previous concentration results) during periods of high sunlight. This creates seasonal influences on $\mathrm{O}_{3}$ production related to trends of sunlight; $\mathrm{O}_{3}$ is produced at higher rates during July than February. In Canada, the "Ozone Season" is defined as May $1^{\text {st }}$ to September $30^{\text {th }}$ (ECCC, 2013b). The lifetime of $\mathrm{NO}_{2}$ is driven by photochemical decomposition; in wintertime, lower sunlight results in longer atmospheric lifetimes of $\mathrm{NO}_{2}$ while in summer, $\mathrm{NO}_{2}$ has a shorter atmospheric lifetime (Boersma et al., 2007). It is expected that the February results be dominated by $\mathrm{NO}_{2}$ related $\mathrm{MB}$, whereas in July, $\mathrm{O}_{3}$ will also have more of a contribution to $\mathrm{MB}$. Due to the relatively short lifetime of $\mathrm{NO}_{2}$ compared to $\mathrm{O}_{3}, \mathrm{NO}_{2}$ has localized concentrations and hence exposure related health impacts, while $\mathrm{O}_{3}$ impacts occur further downwind. Marginal benefits depend on source-receptor relationships and the distribution of population, two factors that change from one resolution to another. Since these two factors vary spatially and are dependent on resolution, MB patterns between resolutions do not always show similar trends. 


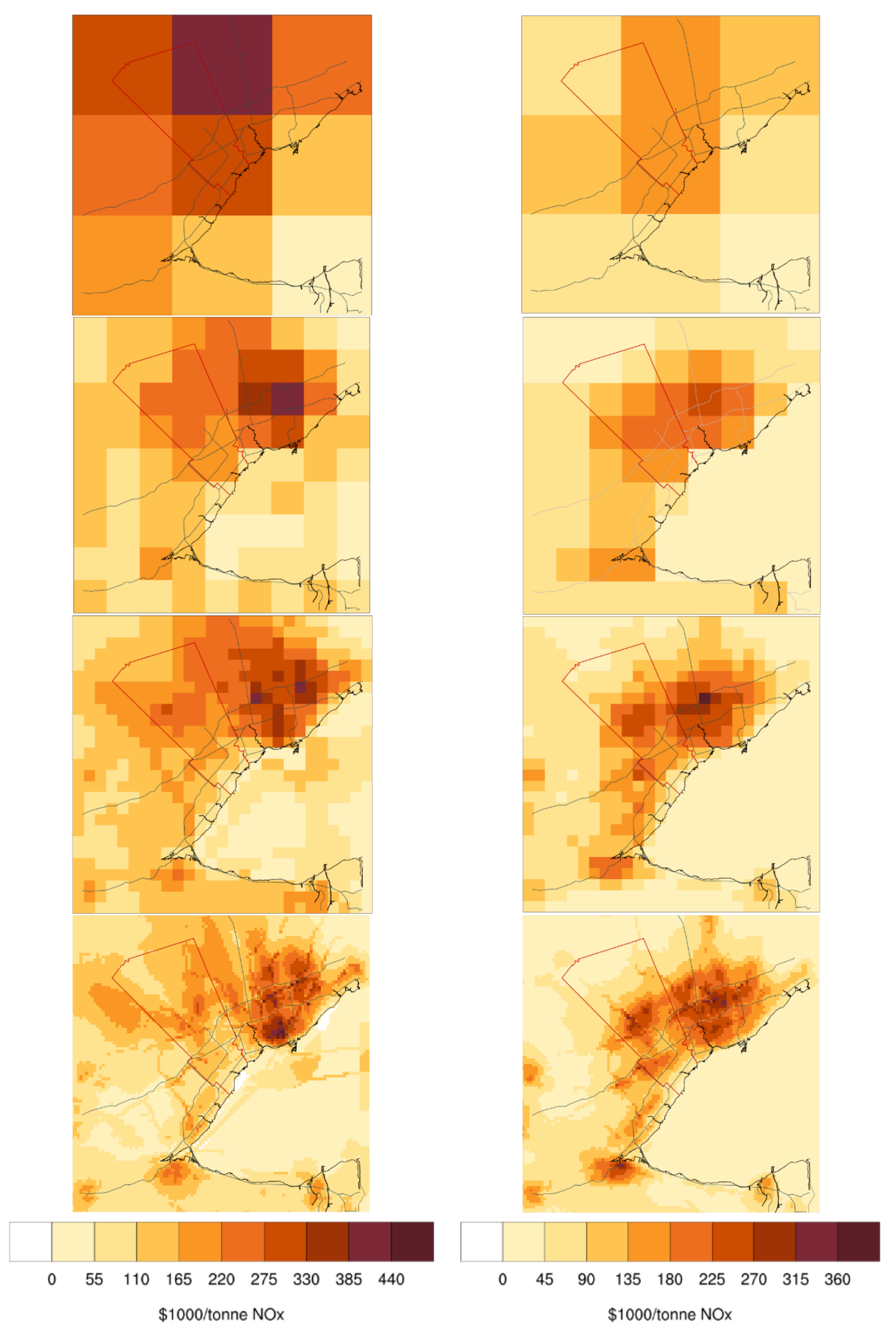

Figure 4-7. July (left) and February (right) MB results at resolutions of 36, 12, 4 and $1 \mathrm{~km}$ (top to bottom). 
In both July and February, the maximum MBs are higher for the fine resolution model, progressively diminishing as the results become coarser (Table 4-2), with the $1 \mathrm{~km}$ resolution maximum being about 1.7 times the $12 \mathrm{~km}$ resolution maximum within both months. These more extreme maxima are anticipated at fine resolutions as less dilution occurs within a grid, for all parameters (emissions, meteorology and population).

It can also be seen that the minimum July MB values at fine resolution are slightly negative, with the negatives diminishing as the resolution becomes coarser until it is no longer present. MB estimates found using CMAQ-adjoint sensitivity by Pappin et al. (2013) suggest that when analyzing $\mathrm{O}_{3}$ exposure during ozone season, a reduction in NOx resulted in a disbenefit in large cities with $\mathrm{NO}_{x}$ inhibited atmosphere, where a reduction in $\mathrm{NO}_{x}$ results in an increase in $\mathrm{O}_{3}$, and hence worsens health impacts. This disbenefit is often cancelled by the associated benefit from increased $\mathrm{NO}_{2}$; however, at higher resolutions this compensation may not happen at the same grid. In July, there are locations in the downtown core that show slight disbenefits at the $1 \mathrm{~km}$ resolution, which diminish at the $4 \mathrm{~km}$ resolution $\mathrm{km}$ and are no longer present at the $12 \mathrm{~km}$ resolution. Cohan et al. (2006) also found that localized pockets of NOx-inhibition were identified at $4 \mathrm{~km}$ resolutions but lost at 12 or $36 \mathrm{~km}$ resolutions. Due to the low concentrations of $\mathrm{O}_{3}$ in February, this trend is not present as NOx inhibition is unlikely.

Table 4-2. Maximum marginal benefit results from each resolution (1, 4, 12 and $36 \mathrm{~km})$.

\begin{tabular}{ccccc} 
& \multicolumn{4}{c}{ Max. MB (\$1000 health benefit / tonne NOx reduction) } \\
\hline $\mathbf{1 ~} \mathbf{~ k m}$ & $\mathbf{4} \mathbf{~ k m}$ & $\mathbf{1 2} \mathbf{~ k m}$ & $\mathbf{3 6} \mathbf{~ k m}$ \\
\hline July & 618 & 378 & 363 & 359 \\
February & 420 & 392 & 256 & 172 \\
\hline
\end{tabular}


MB results between resolutions were further compared using box and whisker plots of grid cells over a common area for both urban and rural locations, for both July (Figure 4-9) and February (Figure 4-10). The urban and rural box and whisker plots were graphed over the same range to highlight the difference in variance between the two land use types. Both July and February plots depict the high variance that occurs in the multiple fine resolution $(4$ and $1 \mathrm{~km}$ ) cells that make up one coarse $(36 \mathrm{~km})$ cell, demonstrating how much spatial detail is lost at coarse resolutions. Both months show that the urban results have a drastically higher range of $1 \mathrm{~km}$ values within a $36 \mathrm{~km}$ area than the rural values, suggesting that fine resolution modelling is of higher importance for results occurring within urban areas. This is in agreement with studies that found near dense urban areas, model resolution has a greater impact on both concentrations (Jang et al., 1995a; Liang \& Jacobson, 2000) and attributed health impacts (Thompson et al., 2013). Due to the high sensitivity of MB to resolution near urban areas, these areas should be modelled with fine resolution. 

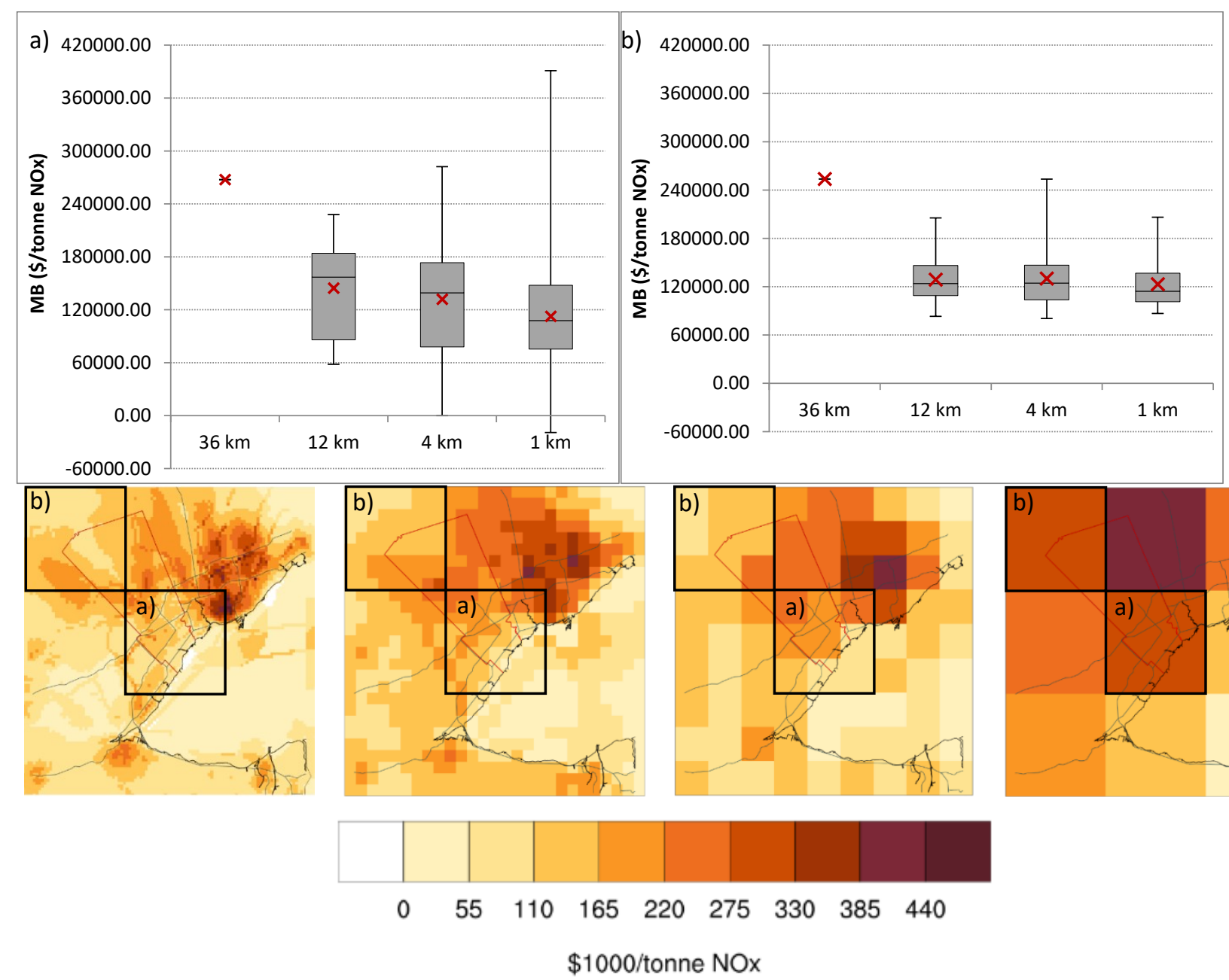

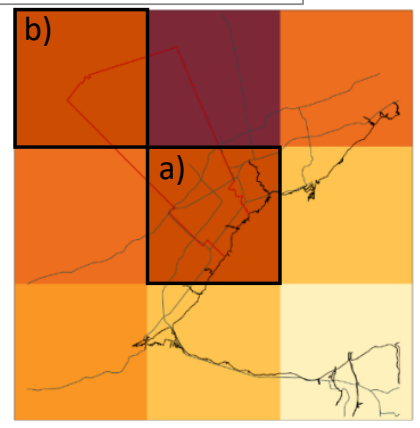

Figure 4-8. July MB resolution comparisons using box and whisker plots at a) a predominantly urban location and at (b) a predominantly rural location. 

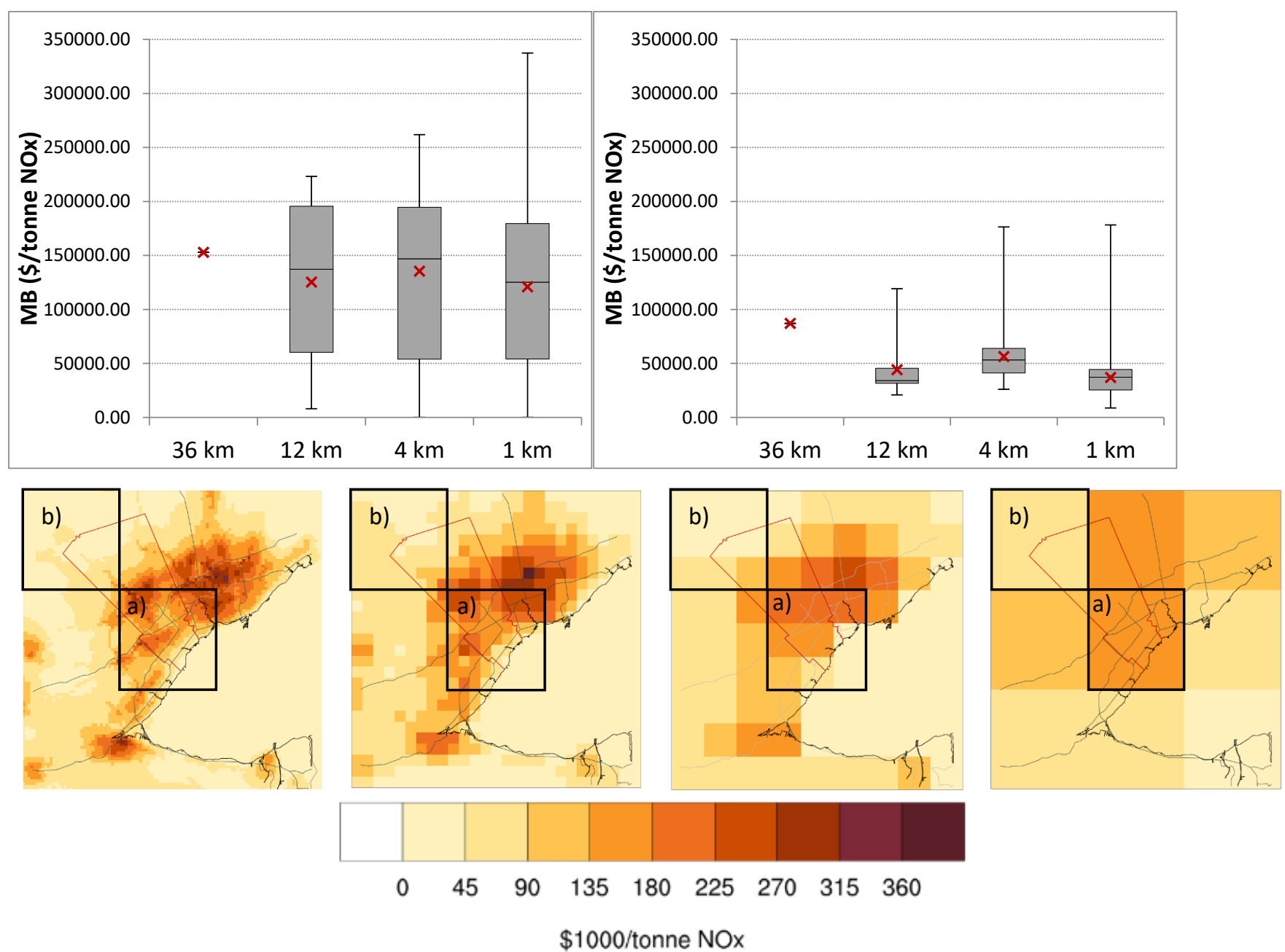

Figure 4-9. February $\mathrm{MB}$ resolution comparisons using box and whisker plots at a) a predominantly urban location and at (b) a predominantly rural location.

Monthly damages ( $\$ /$ month), displayed in Figure 4-10, were estimated from the product of MB and the total monthly NOx emissions. The total emissions across the domain were consistent between resolutions and as such are not a factor influencing the results. These results are quite heterogeneous based on the locations of high NOx emission sources. The results in Figure 4-11 reveal the locations in which both $\mathrm{MB}$ and emissions are high. Since policy is most likely to enforce reduction of high emission sources, the areas of maximums within these plots 
correspond to locations in which emission reductions will have the highest impact. The finer spatial resolution allows for decision makers to efficiently focus efforts of emission reduction where the highest benefits will be achieved. 

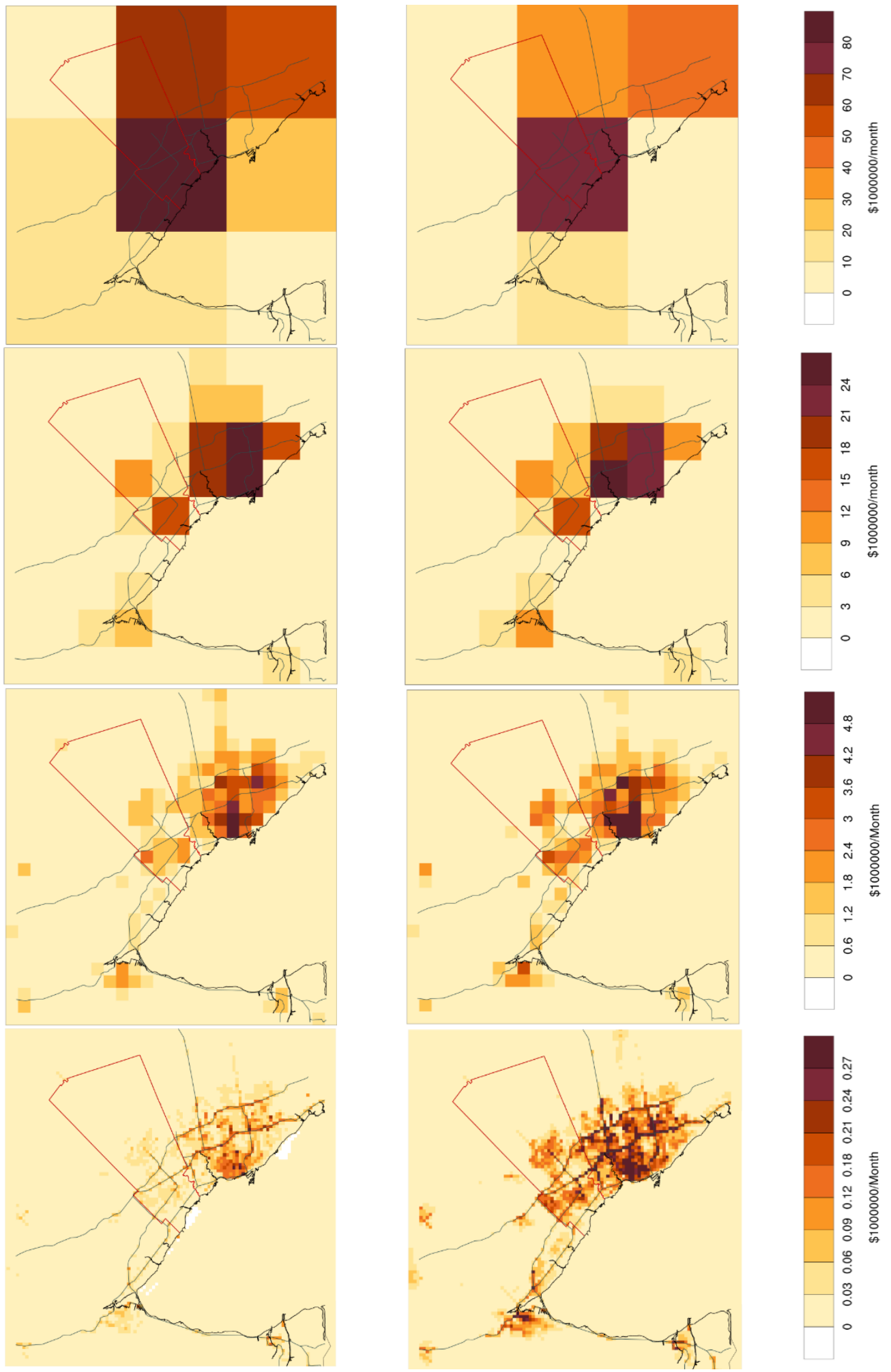

Figure 4-10. Monthly damage result for July (left) and February (right) for 36, 12, 4 and $1 \mathrm{~km}$ resolutions (from top to bottom). 
The higher magnitude maximums seen at the fine resolution does not necessarily indicate that the domain-wide marginal benefits are higher, or that coarse resolution modelling necessarily underestimates total health impacts across the domain, as demonstrated in the total monthly damages between resolutions presented in Table 4-3. Domain-wide total monthly damages (\$/month) were estimated by summing each grid cells' monthly damage results.

In February there is not a high variance in the total monthly damages between resolutions, with a slightly lower estimate at the coarse resolution. Despite the loss in spatial resolution, the coarse resolution total damage results in February did not alter significantly from the fine resolution results.

The July results for total monthly damage had greater result discrepancies between resolutions but remain within the same magnitude. The July MB results decreased as the resolution got finer, which is opposite of the trend seen in February. This may be a result of the $\mathrm{O}_{3}$ related disbenefit that only occurred in July being captured the highest at $1 \mathrm{~km}$, with the extent diminishing as the resolution got coarser. These disbenefits would bring down the domain wide total-damage. The $36 \mathrm{~km}$ July total damages were higher than the other resolutions, suggesting an overestimation. This may be attributed to the fact that $\mathrm{O}_{3}$ sensitivity is often higher when $\mathrm{NO}_{2}$ concentrations are low, and at $36 \mathrm{~km}$ resolution, the $\mathrm{NO}_{2}$ concentration maxima are underpredicted due to inability to represent the high spatial variability in $\mathrm{NO}_{2}$ concentrations (a result of its short lifetime). Both July and February domain-wide total monthly damages predicted for the 4 and $12 \mathrm{~km}$ resolutions were quite similar. 
Table 4-3. Total domain-wide monthly damage results from each resolution (36, 12, 4 and $1 \mathrm{~km})$.

\begin{tabular}{lcccc} 
& \multicolumn{4}{c}{ Total Monthly Damage (\$10^6/ month) } \\
\cline { 2 - 5 } & $\mathbf{1} \mathbf{~ k m}$ & $\mathbf{4} \mathbf{~ k m}$ & $\mathbf{1 2} \mathbf{~ k m}$ & $\mathbf{3 6} \mathbf{~ k m ~}$ \\
\hline February & 248 & 253 & 231 & 206 \\
July & 106 & 274 & 271 & 320 \\
Monthly Avg. & 172 & 264 & 251 & 263 \\
\hline
\end{tabular}

The domain-wide monthly damages predicted at the $36 \mathrm{~km}$ resolution are overestimated in July while underestimated in February, compared to the finer resolution results. Depending on the application of results, it may not always be justifiable due to the trade-offs with computational costs to model at very fine resolution. There is a small variance between results generated at 4 $\mathrm{km}$ and $12 \mathrm{~km}$ within both months, and in February, the $1 \mathrm{~km}$ results also do not vary drastically from the 4 or $12 \mathrm{~km}$ results. Cohan et al.'s (2006) research came to a similar conclusion, as aggregating fine resolution results to coarse resolutions gave consistent $\mathrm{O}_{3}$ sensitivity (to its precursors) results across domains, despite losing pockets of localized NOx inhibition at coarse resolutions. Thompson and Selin (2012) estimated health benefits associated with reductions in modeled $\mathrm{O}_{3}$ concentrations at $36,12,4$ and $2 \mathrm{~km}$ resolutions and found that there was not a significant difference between the 2,4 and $12 \mathrm{~km}$ resolution results but the $36 \mathrm{~km}$ results may have over-predicted. If the purpose of modelling is for domain total results and high resolution spatial representation is not necessary, then choosing a resolution as fine as $1 \mathrm{~km}$ may not be justified.

\subsubsection{Discussion of Limitations}

The research presented within this study is subject to several limitations, sourcing from both the methodology and the scope of work. Atmospheric models contain inherent uncertainties 
when it comes to representing complex atmospheric operations. Uncertainties in simulated concentrations are thought to be heavily impacted by uncertainties in the inputted meteorology and emissions, while also impacted by uncertainties within the model chemical mechanisms (Russell and Dennis 2000). There is also inherent uncertainty within the epidemiological relationships and data used within this research.

The work involved analyzing the $\mathrm{MBs}$ associated with exposure to $\mathrm{O}_{3}$ and $\mathrm{NO}_{2}$ combined. Analyzing $\mathrm{O}_{3}$ and $\mathrm{NO}_{2}$ separately would be beneficial as the two species behave differently in the atmosphere and hence are influenced by resolution differently, as the concentration results presented suggest. The influence of resolution on $\mathrm{MB}$ due to $\mathrm{PM}_{2.5}$ exposure has not been analyzed due to the adjoint of aerosols only being developed at the time of the work. Predicting $\mathrm{MB}$ for the speciated $\mathrm{PM}_{2.5}$ compounds would aid in strengthening the link between resolution and $\mathrm{MB}$ estimates.

The short episodes modelled and expanded annually is another limitation of this research. Inter-annual variability occurring during the other periods outside the modelled episode may alter the results. Modelling a full year would reduce these uncertainties, however, be extremely costly in terms of time and computational resources.

The domain within this study contained an urban core (the Greater Toronto Area) along the shore of a large body of water (Lake Ontario). This topography influenced the results, as averaging occurred between parameters on land and over water, some of which had strong 
gradients between the two (i.e. population and emissions). As such, these results may not be applicable to all other domains, especially those which are completely in-land. It is suggested that future work look at alternative domains with varying land-use types.

The concentration response function applied to estimate mortality assumes a uniform response to all populations, which may not be accurately representative. Due to this high uncertainty associated with the human health response to air pollution, health estimates generated from fine resolution models $(<36 \mathrm{~km})$ may not be more accurate than those created from coarse resolutions ( $\geq 36 \mathrm{~km}$ ) (Arunachalam et al., 2011; Thompson \& Selin, 2012).

The MB values presented within this paper may be underestimated as they do not consider the health impacts occurring outside of the $1 \mathrm{~km}$ domain, which would be caused by long-range transport of pollution sourcing from within the domain. This would be more so prominent for $\mathrm{O}_{3}$, a longer-live species than $\mathrm{NO}_{2}$. Due to the high computational cost associated with fine grid resolution modelling, it is difficult to expand the domain of the fine resolution model. A 2-way nested model could help resolve this underestimation where the entire outer domain has forcing applied.

\subsection{Conclusions}

The impact of model resolution on predicted concentrations of $\mathrm{O}_{3}, \mathrm{NO}_{2}$, and $\mathrm{PM}_{2.5}$, and $\mathrm{MB}$ estimates were investigated through repeated model runs at progressively refined resolutions of $36,12,4$ and $1 \mathrm{~km} . \mathrm{MB}$ in this research was parameterized as mortality due to combined 
long-term $\mathrm{O}_{3}$ and $\mathrm{NO}_{2}$ exposure with respect to $\mathrm{NOx}$ reductions. Results show that model resolution had the largest influence on concentrations of $\mathrm{PM}_{2.5}$, followed by $\mathrm{NO}_{2}$ and had the least influence on $\mathrm{O}_{3} . \mathrm{MB}$ results were more impacted in July than in February, which may be attributed to the increased photochemical reactions altering the production and lifetime of both $\mathrm{NO}_{2}$ and $\mathrm{O}_{3}$. The fine resolution results for both concentrations and $\mathrm{MB}$ show better spatial distribution and geographical representation.

It was expected that as model resolution became coarser, that maximum concentrations would diminish due to averaging within each grid cell. This held true for all results in February but was only true for $\mathrm{PM}_{2.5}$ in July. Maximum 24-hour average $\mathrm{NO}_{2}$ saw a decrease between 12 and 36 $\mathrm{km}$ in July, dropping from 23.1 to $19.1 \mathrm{ppb}$, but saw an increase from 1 to $12 \mathrm{~km}$, from 20.6 to $23.1 \mathrm{ppb}$. Maximum average $\mathrm{DM} 8 \mathrm{~A} \mathrm{O}_{3}$ concentrations also increased as the resolution became coarser, increasing from $60.8 \mathrm{ppb}$ to $67.6 \mathrm{ppb}$ from 1 to $36 \mathrm{~km}$. It is suggested that a resolution of $36 \mathrm{~km}$ is not sufficient to model primary short-lived species as they cannot properly depict localized gradients.

The MB results indicate that a resolution of $36 \mathrm{~km}$ does not adequately represent spatial variance, especially for areas within urban cores, as displayed in box and whisker plots comparing the $36 \mathrm{~km}$ results with the 12,4 , and $1 \mathrm{~km}$ results over the same area. MB results had higher maximums at fine resolutions, which progressively diminished as the resolution became coarser. The maximum MB results were $\$ 618000, \$ 378000, \$ 363000$, and $\$ 359000$ per tonne NOx reduced for 1, 4, 12 and $36 \mathrm{~km}$, respectively in July and were $\$ 420000$, \$392 
$000, \$ 256000$, and $\$ 172000$ per tonne NOx reduced for $1,4,12$ and $36 \mathrm{~km}$, respectively in February. The improved MB spatial distribution at fine resolutions allows for the isolation of specific emission sources which may have the highest benefit from reducing NOx emissions. Modelling at fine resolution can also be beneficial for urban development, specifying areas in which industry would result in a higher health impact. This can help inform decision makers to target specific locations for emission reductions, optimizing the efficiency of the corresponding health benefits.

Resolution impacts on MBs had seasonal influences, as July results are anticipated to be more influenced by $\mathrm{O}_{3}$ related $\mathrm{MB}$ than February. The impacts of resolution on the $\mathrm{MB}$ estimates were more prominent in July than February, possibly a result of the temporal impacts of the atmospheric lifetime of $\mathrm{NO}_{2}$ and the photochemical production of $\mathrm{O}_{3}$. It is expected that the July results be more influenced by $\mathrm{O}_{3}$ related $\mathrm{MB}$ than those in February, as validated by the higher July $\mathrm{O}_{3}$ concentrations. The $\mathrm{O}_{3}$ related disbenefit that occurred in July due to a NOxinhibited atmosphere (in the downtown Toronto core) was captured the highest at $1 \mathrm{~km}$, with the extent diminishing as the resolution got coarser. This led to a higher domain-wide total damage at coarse resolutions, suggesting that the inability to capture these disbenefits may result in over-estimation of the MB in downtown cores.

When domain-wide total mortality was estimated, it was found that the results did not substantially change between resolutions in February, while in July, the 4 and $12 \mathrm{~km}$ results were very close, while the $1 \mathrm{~km}$ results were noticeably lower and the $36 \mathrm{~km}$ noticeably higher. 
As such, it can be suggested that if the purpose of the study is to estimate total domain-wide health impacts, a resolution as fine as $1 \mathrm{~km}$ may not be required, however, a resolution of 36 $\mathrm{km}$ may be too coarse to properly simulate results. The total damage results not changing magnitude between model resolutions suggest that justification of the trade-off of computational resources for better spatial representation is subject to the intent of usage of the results, along with the scope of work. For example, in rural areas, it may not be beneficial to model at a resolution as fine as 1 or even $4 \mathrm{~km}$. 


\subsection{Assessing the Impact of CMAQ Input Resolution on Concentration and Associated Health Impacts}

This chapter is in the format of a draft manuscript. It contains original research with Melanie Fillingham as the main contributor $(80 \%)^{2}$.

\section{Abstract}

Health impacts due to exposure to air pollution can be predicted by pairing air quality model $(A Q M)$ results (i.e. concentrations) with pollution exposure and epidemiological relationships. The accuracy of these predictions often depends, amongst other factors, on the grid resolution of both the model and its respective inputs. This research analyzes the impact of model input (i.e. meteorology, emission, and population) resolution on ozone $\left(\mathrm{O}_{3}\right)$, nitrogen dioxide $\left(\mathrm{NO}_{2}\right)$ and fine particulate matter $\left(\mathrm{PM}_{2.5}\right)$ concentrations and associated health related impact predictions by varying the resolutions of model inputs. Input resolutions were varied between combinations of 1 and $4 \mathrm{~km}, 4$ and $12 \mathrm{~km}$, and 12 and $36 \mathrm{~km}$.

The research was performed using CMAQv5.0.2 paired with information from the Air Quality Benefits Assessment Tool. Statistical analyses between scenario of various resolution inputs scenario were done to test influence of the coarse inputs. Our analysis suggests that the sensitivity to specific input resolutions is dependent on the species, as well as the type of input. For example, $\mathrm{O}_{3}$, a relatively long-lived secondary photochemically produced compound, had a

\footnotetext{
${ }^{2}$ Co-authors on this work include: Burak Oztaner, Shunliu Zhao, Amir Hakami (Carleton University); Amanda Pappin (Statistics Canada); Wayne Boulton, Greg Conley, Martin Gauthier, Jeff Lundgren, Julia Veerman,Carol McClellen (RWDI Inc.); Louise Aubinm, Eric Tran, Kim McAdam (Regional Municipality of Peel Public Health Department); and the CMAQ Adjoint Development Team
} 
greater sensitivity due to the resolution of meteorology than emissions, whereas $\mathrm{NO}_{2}$, a primary compound, and $\mathrm{PM}_{2.5}$, a portion in which is primary, both relatively short-lived compounds, were more sensitive to resolution of emissions. Health impacts for all three species were dominated by the influence of population resolution, followed by the input resolution influences seen from concentration predictions.

Key Words: grid resolution, air quality health impacts, air quality model performance, input representation.

\subsection{Introduction}

Air pollutants, such as nitrogen dioxide $\left(\mathrm{NO}_{2}\right)$, ozone $\left(\mathrm{O}_{3}\right)$, and fine particulate matter $\left(\mathrm{PM}_{2.5}\right.$, particulate matter with an aerodynamic diameter $<2.5 \mu \mathrm{m})$, contribute to negative impacts on human health, the environment, and crop production (Burnette et al., 2004; Bell et al., 2004; Jerret et al., 2009; Mills et al., 2011;). Three-dimensional Eulerien (i.e. gridded) chemical transport models (CTMs) can be used to analyze pollution trends and are valuable in mitigation efforts through regulatory and policy applications. One such CTM is the US Environmental Protection Agency's (EPA) Community Multi-scale Air Quality (CMAQ) model. By pairing CTMs with pollution exposure and epidemiological relationships, health impacts due to pollution exposure can be estimated. The accuracy of this method often depends, amongst other factors, on model resolution and its respective inputs (e.g. meteorology, emissions, and population).

Numerous studies have evaluated the impact of model grid resolution on CTMs (Wild \& Prather, 2014; Punger \& West, 2013; Micea, et al., 2010) and the associated health impacts of 
pollution exposure (Arunachalam et al., 2011; Thompson and Selin, 2012; Li et al., 2016). The results from these studies have demonstrated that grid resolution impacts predictions, and often found that high (fine) resolution modelling better predicts actual concentrations which is expected to improve health estimate accuracy. Coarse grid resolutions are prone to inaccuracy due to resolutions beyond what is suitable to capture the spatial variability in emissions, meteorology, and populations. This causes a trade-off between computational cost and accuracy. Most of the research on the impacts of grid resolution performed to date have evaluated the overall impacts of resolution on CTMs, using consistent resolution between all inputs (e.g. meteorology, emissions and population) and running iterations at varying resolutions. Fewer studies have studied how the resolution of individual model inputs impacts CTM results and the paired health impacts independently (Wu et al. 2008a, 2008b; Menut, Coll \& Cautenet, 2005; Hodnebrog, Stordal \& Berntsen, 2011). Analyzing the individual impact of each input parameter can aid in optimizing which inputs would most benefit from being prepared and processed at high resolution.

Within CTMs, emissions are instantaneously and uniformly averaged across the volume of each grid cell, resulting in artificial dilution. This artificial dilution is especially significant in locations of high spatial gradients, such as over urban regions or near large emission sources, in which coarse resolutions may not be able to capture the geographical inhomogeneities. This directly impacts the concentrations of primary species, such as $\mathrm{NO}_{2}$ and some $\mathrm{PM}_{2.5}$ species, and precursors of secondary species, such as nitrogen oxides (NOx, composed of $\mathrm{NO}_{2}$ and nitrogen monoxide, NO) and VOCs involved in the formation of $\mathrm{O}_{3}$ (Jang et al., 1995; Cohan et al., 2006; 
Wu et al., 2008a \& 2008b; Hodnebrog, Stordal and Berntsen, 2011). The highly non-linear $\mathrm{O}_{3}$ formation chemistry and its sensitivity to precursors causes inaccurate representation when precursor concentrations are diluted due to coarse emissions. Coarse model emissions cannot sufficiently simulate the extent of NO-titration of $\mathrm{O}_{3}$ in urban areas, often overestimating $\mathrm{O}_{3}$ concentrations (Jang et al., 1995; Hodnebrog, Stordal and Berntsen, 2011; Im \& Kanakidou, 2012). $\mathrm{NO}_{2}$ and $\mathrm{PM}_{2.5}$ maximum concentrations, occurring near emission sources, diminish at coarse resolutions (Jang et al., 1995; Wu, et al., 2008a).

CTMs rely on meteorology data for advection fields that drive pollutant transportation, aerosolcloud chemistry, photochemical reactions (a function of solar radiance and cloud cover), temperature dependent chemistry, land-use parameters, and for the deposition of species within the model. This reliance on meteorology make CTMs sensitive to the accuracy and resolution of meteorological inputs. The horizontal resolution of the meteorological input into chemical transport models has shown to influence air quality results (Menut, Coll \& Cautenet, 2005; Queen \& Zhang, 2008; Wu et al., 2008a; Wolke et al., 2012). Additionally, there are a profuse amount of studies which have demonstrated the impacts of resolution on meteorological model results, but do not pair these with CTMs (Stohl et al., 1995; Weisman et al., 1997; Grabowski et al., 1998; Gallus, 1999; Mass, Ovens, \& Westrick., 2002; Colle \& Olson, 2003; Leung \& Qian, 2005; Gao et al., 2006; Rauscher et al., 2009; Ramu et al., 2016).

Coarse resolution meteorological modelling can result in significant errors in heavy precipitation events (Weisman et al., 1997; Grabowski et al., 1998, Mass, Ovens \& Westrick, 
2002; Leung \& Qian, 2002) and slower convection evolution (Weisman et al., 1997, Mass, Ovens \& Westrick, 2002), factors which influence the modeling of precipitation, a pollutant scavenging process. Heavy precipitation can be diluted at coarse resolution modelling. Wu et al. (2008a and 2008b) found that pollutant concentrations of $\mathrm{PM}_{2.5}, \mathrm{SO}_{4}{ }^{2-}$ and $\mathrm{NO}_{3}{ }^{-}$had higher sensitivity to the grid resolution of meteorology (specifically cloud fractions and precipitation) than compared to those of emissions. They also found good model performance at $4 \mathrm{~km}$ resolution for meteorological variables and $\mathrm{O}_{3}$ mixing ratios, showing better performance than at $12 \mathrm{~km}$ and $36 \mathrm{~km}$ resolutions.

Overall, past research reveals that CTMs run with fine meteorology ultimately have better pollutant predictions than those using coarse meteorology (Menut, Coll \& Cautenet, 2005; Queen \& Zhang 2008). The resolution of meteorology inputs has stronger influences on CTMs in coastal regions or those with complex terrain (e.g. mountain, valley) than in simple terrain areas due to the strong spatial gradients. Fenger (2005) and Palau et al. (2005) suggest that for complex terrain coastal regions, lower layer pollution behaviour can be poorly simulated due to the strong influence resolution has on integral advection and turbulent dispersion.

Upon review of available literature, there was a lack of studies evaluating how resolution of specific CTM inputs influence exposure related health impacts. Resolution influences on CTM concentration results would carry over to impacting health estimate results. It could easily be mistaken that the resolution impacts on concentration would translate proportionately to the resolution impacts on health estimates. This is not necessarily the case as population gradients 
do not always spatially match with the gradients formed by emissions or meteorology, nor are they temporally dynamic within each grid cell the way that the other parameters are. The exposure equation relating concentrations and health impacts is directly proportional to the population, making it highly sensitive to population resolutions.

In this study, the influence of CTM input resolution on CMAQ concentration predictions of $\mathrm{O}_{3}$, $\mathrm{NO}_{2}$, and $\mathrm{PM}_{2.5}$ and associated exposure related health impacts were evaluated. This was achieved by altering the resolution of the model inputs (emissions, meteorology, and population) through multiple model iterations. Model input resolutions were varied between combinations of 1 and $4 \mathrm{~km}, 4$ and $12 \mathrm{~km}$, and 12 and $36 \mathrm{~km}$. The scenarios with all fine resolution inputs were taken as the "base models" in which the coarse resolution input scenarios were statistically compared to. The main difference, amongst others, making this research unique from other studies such as Wu et al. (2008) and Hodnebrog et al. (2011), is that it involves analyzing input resolution influence on health impacts related to pollution exposure.

\subsection{Methodology}

\subsubsection{Input Generation Process}

The CMAQ input data subject to analysis in this study include emissions (excluding point sources as plume rise that are processed in-line), meteorology, and the population (used for exposure related health impacts). The resolution of the CTM itself and its impact on numerical integration was also analyzed. The coarse resolution inputs for each scenario were developed by aggregating or averaging, depending on whether the parameter is extensive or intensive, the 
original fine resolution data up to the coarse resolution using the CMAS spatial allocator tool.

Due to the CMAQ requirement for consistent resolution inputs, the coarse input data was then "re-gridded" (i.e. scaled down) back down to the respective fine resolution. Whether a parameter was averaged or aggregated depended on whether it was an intensive (e.g. temperature) or an extensive parameter (e.g. emissions). The code had to be altered for the METDOT3D and GRIDDOT2D files, as this data was from the corner fields of each cell, opposed to the other meteorological files (METCRO2D, METCRO3D, GRIDCRO2D, GRIDCRO3D) which were from cell centers.

\subsubsection{Case Studies}

The impacts of the model input data resolution were analyzed for each input parameter, independently and combined, by running all combinations of coarse and fine input data. By exclusively modelling with a single input at a coarse resolution while the remainder of inputs are at a fine resolution, the impacts of resolution on that specific input parameter can be evaluated. An example of this would be to input coarse resolution emissions while meteorology, the CTM and population, remain at fine resolution. Synergetic impacts of numerous coarse resolution inputs were also assessed; every combination between fine and coarse inputs were evaluated.

Table 5-1 summarizes the combinations of coarse/fine resolution input data used within this study, with a small italicized letter indicating a fine resolution (e.g. $e$ indicates emissions are fine) whereas a capital non-italicized letter indicates a coarse resolution (e.g. E indicates emission are coarse). In this table, E stands for emissions, $\mathrm{M}$ for meteorology, C for the model 
(CMAQ) and $\mathrm{P}$ for population. These combinations of fine/coarse resolution input data were used between resolutions of 1 and $4 \mathrm{~km}, 4$ and $12 \mathrm{~km}$, and 12 and $36 \mathrm{~km}$, resulting in a total of 24 different runs, i.e., Table 5-1 was then repeated for various resolution combinations of $1 / 4$, $4 / 12,12 / 36,1 / 12,1 / 36$, and $4 / 36$.

Table 5-1 Input resolution combinations assessed, where an italicized lower-case letter indicates a fine resolution input and a non-italicized upper-case letter indicates a coarse resolution input.

\begin{tabular}{|c|c|c|c|}
\hline & \multicolumn{3}{|c|}{ Process / Input Parameter } \\
\hline & Emissions & $\begin{array}{c}\text { Meteorology } \\
\text { (WRF) }\end{array}$ & Population \\
\hline \multirow{8}{*}{ 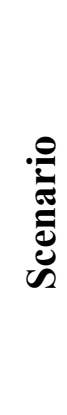 } & $e$ & $m$ & $p$ \\
\hline & $e$ & $m$ & $\mathrm{P}$ \\
\hline & $e$ & M & $\mathrm{P}$ \\
\hline & $e$ & M & $p$ \\
\hline & $\mathrm{E}$ & M & $\mathrm{P}$ \\
\hline & $\mathrm{E}$ & $m$ & $\mathrm{P}$ \\
\hline & $\mathrm{E}$ & M & $p$ \\
\hline & $\mathrm{E}$ & $p$ & $p$ \\
\hline
\end{tabular}

\subsubsection{Model Parameters}

The modelling for this study was performed using CMAQ, version 5.0.2. The model domains within this evaluation are displayed in Figure 5-1. The innermost domain is that of the original 1 $\mathrm{km}$ resolution data, followed by the original $4 \mathrm{~km}$ resolution data, and the outer most being that of the original $12 \mathrm{~km}$ resolution data, in which the fine scenarios are based on. The domain belonging to the fine resolution model is the domain each scenario set is modelled (e.g. the 1 and $4 \mathrm{~km}$ scenario is modelled over the $1 \mathrm{~km}$ domain, whereas the 4 and $12 \mathrm{~km}$ scenario is modelled over the $4 \mathrm{~km}$ domain). Only the predictions within the common domain between all resolutions (i.e. the inner-most $1 \mathrm{~km}$ domain) were compared for parameter consistency. 


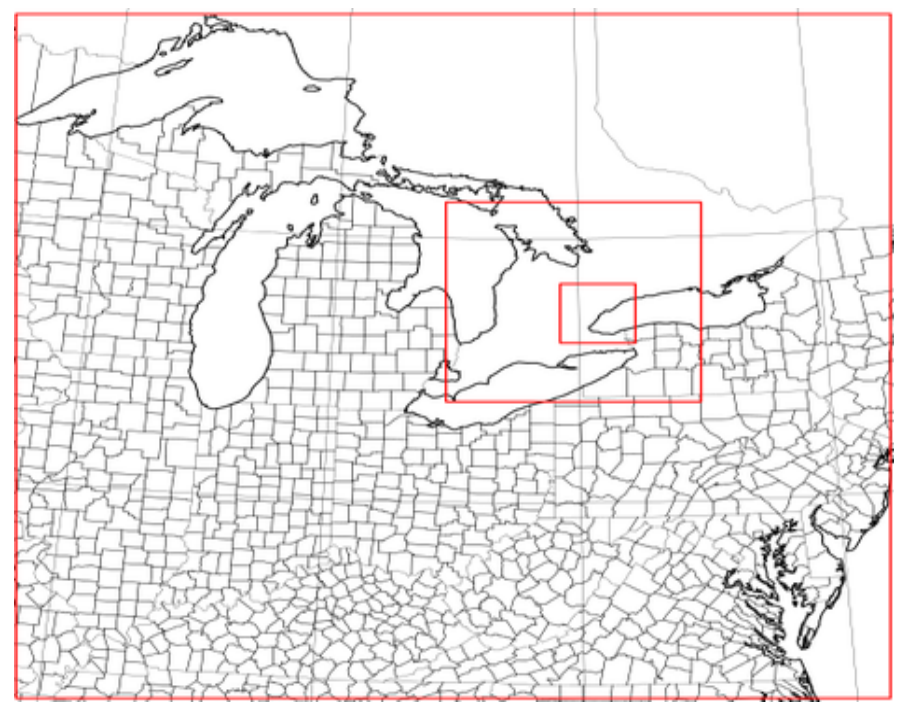

Figure 5-1 Domains within the analysis, inner to outer domains for the original 1, 4 and $12 \mathrm{~km}$ model domains, respectively.

The emissions and meteorology were run for each base resolution (1, 4 and $12 \mathrm{~km})$ over the domains displayed in Figure 5-1. The coarse emission and meteorology data was generated from the fine data, as described in Section 5.2.1. The U.S. emissions were developed from the 2008 National Emission Inventory (NEI). The Canadian emissions were developed from Environment Canada's (EC) 2010 National Pollution Release Inventory (NPRI) for point sources, from the 2011 National Agri-Environmental Standards Initiative for area sources, and the remaining area and mobile emissions from EC's 2006 Criteria Area Contaminants (CAC). The Weather Research and Forecast (WRF) model version 3.4.1 was used to create the meteorological inputs which were processed for each resolution (nested model) (Skamarock et al., 2008). Due to the high quantity of model scenarios, each scenario was only simulated for 2 days, July $9^{\text {th }}$ and $10^{\text {th }}, 2012$. The short simulation period is a limitation of the research, discussed in Section 5.3.3. This analysis focuses specifically on the resolution impacts on concentrations and health impacts of $\mathrm{O}_{3}, \mathrm{NO}_{2}$, and $\mathrm{PM}_{2.5}$. 


\subsubsection{Exposure Health Predictions}

The health impacts within this study are parameterized as mortality due to long-term exposure to $\mathrm{O}_{3}, \mathrm{NO}_{2}$, and $\mathrm{PM}_{2.5}$, presented independently. The CMAQ modelled concentration results were linked to human health using the following pollution exposure equation (equation 3.15), applied to each grid cell:

$$
\Delta M(\$)=M_{0} \times P \times V_{S L}\left(1-e^{-\beta \Delta C}\right)
$$

$M_{0}$ is defined as the baseline non-accidental mortality rate for the population aged twenty-five plus obtained from Health Canada's Air Quality Benefits Assessment Tool (AQBAT) (Judek et al., 2006) for each Census Division. P is the population aged twenty-five plus, obtained from Statistics Canada (Census Dissemination Area) from the 2011 Census, and includes only the population aged twenty-five plus. The $V_{S L}$ is an economic valuation of a statistical life, based off the amount one is willing to pay to reduce probability of death. It is frequently represented as

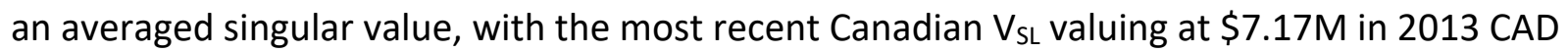
(Chestnut \& Civita, 2009). $\beta$ is an epidemiological value known as the concentration response factor (CRF) which represents the change in mortality seen due to a change in pollutant concentration. The CRF is dependent on both the species of analysis and the epidemiology scenario being studied such as short-term or long-term (chronic) exposure, morbidity or mortality, all-cause or non-accidental, and/or impacts relating to specific disease (e.g. cardiovascular). Within this research, the CRFs applied were related to non-accidental mortality due to chronic exposure to $\mathrm{O}_{3}, \mathrm{NO}_{2}$, and $\mathrm{PM}_{2.5}$, for the population twenty-five years old plus. The CRFs applied were: $0.27 \%$ change in mortality (non-accidental) /ppb change of daily maximum 8-hour average $\mathrm{O}_{3}$ (DM8A), $0.59 \%$ change in mortality (non-accidental) /ppb change 
$\mathrm{NO}_{2}$ (daily average), and $2.7 \%$ change in mortality (non-accidental) $/ \mu \mathrm{g} / \mathrm{m}^{3}$ change $\mathrm{PM}_{2.5}$ (daily average) (Crouse et al., 2015). $\Delta \mathrm{C}$ is a change in pollutant concentration, over the respective averaging period as the $\mathrm{CRF}$ (i.e. DM8A for $\mathrm{O}_{3}$ and daily 24-hour average for $\mathrm{NO}_{2}$ and $\mathrm{PM}_{2.5}$ ). In this research, the mortality at a consistent concentration was analyzed, and as such, the $\Delta C$ was the same as concentration, i.e. concentration above a zero baseline.

\subsection{Results and Discussion}

To keep the presentation of results concise and to focus on the most prominent impacts, only selected figures have been presented within this paper. The remainder of result figures are presented within the supplementary material.

\subsubsection{Concentration Results}

The concentration results were processed by the averaging periods corresponding to their health standards; daily 24-hour average for $\mathrm{NO}_{2}$ and $\mathrm{PM}_{2.5}$, and daily maximum 8-hour average for $\mathrm{O}_{3}$. Comparisons were made between the coarse input scenario results and the original allfine input results to determine the extent of resolution impacts. These comparisons include difference figures, root mean squared difference (RMSD) values, and scatter comparison plots.

\subsubsection{Ozone Concentration Results}

Coarse emissions caused a decrease in the maximum $\mathrm{DM} 8 \mathrm{~A} \mathrm{O}_{3}$ concentration for all resolution combinations (Figure 5-2), with the largest decrease between 12 and $36 \mathrm{~km}$ resolution emissions (5\% decrease from $65.4 \mathrm{ppb}$ to $62 \mathrm{ppb}$ ) and the least decrease between 1 and $4 \mathrm{~km}$ ( $<1 \%$ decrease from $50.3 \mathrm{ppb}$ to $50.2 \mathrm{ppb}$ ). Difference plots between the concentrations generated for the coarse resolution scenarios and those for all-fine resolutions are displayed 
within the supplementary material, Figures 5-14, 5-16 and 5-18 for the 12 and $36 \mathrm{~km}, 4$ and 12 $\mathrm{km}$, and 1 and $4 \mathrm{~km}$ scenarios, respectively. From both the difference plots and the scatter plots presented within Figure 5-3, it is found that several of the original $12 \mathrm{~km}$ cells with the highest $\mathrm{DM} 8 \mathrm{~A} \mathrm{O}_{3}$ concentrations were underpredicted when using $36 \mathrm{~km}$ emissions. This trend is not prominent for the 1 and $4 \mathrm{~km}$ and 4 and $12 \mathrm{~km}$ resolution scenarios, despite the slight decrease in their maximum.

Ozone is a secondary species formed through photochemical reactions of NOx and VOCs. The lower maximum $\mathrm{O}_{3}$ concentrations produced when emissions are as coarse as $36 \mathrm{~km}$ may be a result of $\mathrm{O}_{3}$ precursors dilution, reducing the formation of $\mathrm{O}_{3}$. In result, this causes higher $\mathrm{O}_{3}$ precursors in surrounding areas, which can result in incorrect high $\mathrm{O}_{3}$ formation within these areas. Coarse emissions may cause dilution of high NOx emissions to NOx limited regions, causing lower NO-titration of $\mathrm{O}_{3}$ and hence over-prediction, conclusions found by Hodnebrog et al. (2011), Jang et al. (1995) and Im and Kanakidou (2012). Figure 5-3 shows many of the mid to lower range $\mathrm{O}_{3}$ concentrations being over-predicted at coarse emissions for the 12 and $36 \mathrm{~km}$, and the 4 and $12 \mathrm{~km}$ scenario; a trend not consistent for the 1 and $4 \mathrm{~km}$ scenario. The results show that emissions at resolutions of both 12 and $36 \mathrm{~km}$ impact predicted $\mathrm{O}_{3}$ concentrations, over-estimating the total pollutant mass over the domain compared to the fine resolution results (higher weight of values above the 1:1 trend-line in Figure 5-3). The overprediction of lower concentrations at coarse resolutions is consistent with findings by Jang et al. (1995) who found that NO titration is underpredicted at coarse resolutions. Cohan et al. 
(2006) found that $12 \mathrm{~km}$ resolutions were not fine enough to pick up the localized pockets of NOx inhibition, which $4 \mathrm{~km}$ resolutions could.

Hodnebrog et al. (2011) studied the impacts of emission resolution on CTM results by varying input emission resolutions between 9,27 , and $81 \mathrm{~km}$, while the CTM resolution was held content. They found that there was a $12 \%$ increase in net $\mathrm{O}_{3}$ over the domain when using 81 $\mathrm{km}$ instead of $9 \mathrm{~km}$. The results found within this research also found that net $\mathrm{O}_{3}$ concentrations increased when resolution of emissions increased from 12 to $36 \mathrm{~km}$, with a net increase of $4.9 \%$ between 12 and $36 \mathrm{~km}$.

Coarse meteorology caused the maximum $\mathrm{DM}_{8 \mathrm{~A}} \mathrm{O}_{3}$ concentrations in the 12 and $36 \mathrm{~km}$ and 1 and $4 \mathrm{~km}$ scenarios to increase (Figure 5-2). Figure 5-3 shows that $\mathrm{O}_{3}$ concentrations at all resolution combinations were more impacted by meteorology than emissions, as evident by the large variance from the $1: 1$ trend-line. $\mathrm{As}_{3} \mathrm{O}_{3}$ is a secondary species in which the formation is reliant on meteorology, it is expected that it be sensitive to the resolution of meteorology.

Metrological conditions, such as humidity, temperature, and wind speeds, alter drastically over land and over water. The grid cells which had the highest impacts were those with area spanning both land and water, most evident in Figures 5-14, 5-16 and 5-18. The averaging of meteorology between land and water at coarse resolutions may cause decreases in temperature over land and increases over water (increasing the $\mathrm{O}_{3}$ production over the water and hence decreasing it over land). Research has demonstrated that temperature and wind 
predictions are considerably improved when resolutions are decreased from $36 \mathrm{~km}$ to $12 \mathrm{~km}$ (Mass, Ovens and Westrick, 2002). There was no precipitation within the 2-day period of study, which would have a large impact on pollution scavenging.

The combination of coarse inputs for both meteorology and emissions had the highest impact on concentrations. The results are not the exact values as if the differences of both the individual coarse emissions and coarse meteorology were accumulated and applied to the original, as expected as the non-linear production of $\mathrm{O}_{3}$ alters based on these parameters.

The impact of the resolution of the CTM itself can be analyzed by the comparison of the base consistent resolution results (e.g. all $12 \mathrm{~km}$ ) with the coarse emission and meteorology results in the subsequent finer resolution combinations (e.g. 4 and $12 \mathrm{~km}$ ). The only difference in these cases is the resolution of the model, as inputs are equal (all the coarser resolution). The resolution of the numerical discretization has a large impact on $\mathrm{O}_{3}$ concentrations when going from a $1 \mathrm{~km}$ to $4 \mathrm{~km}$ resolution, as both magnitude of concentrations and the trends alter drastically. Altering the resolution of the CTM from $4 \mathrm{~km}$ to $12 \mathrm{~km}$ did not have a large impact, as spatial trends and the magnitude of results are quite similar. The net emissions across the domain were consistent between resolutions and as such were not a factor. A resolution as fine as $1 \mathrm{~km}$ may not have been able to accurately represent the complex $\mathrm{O}_{3}$ production chemistry. The mass across the $1 \mathrm{~km}$ domain is notably lower than across the 4 or $12 \mathrm{~km}$ domains. 

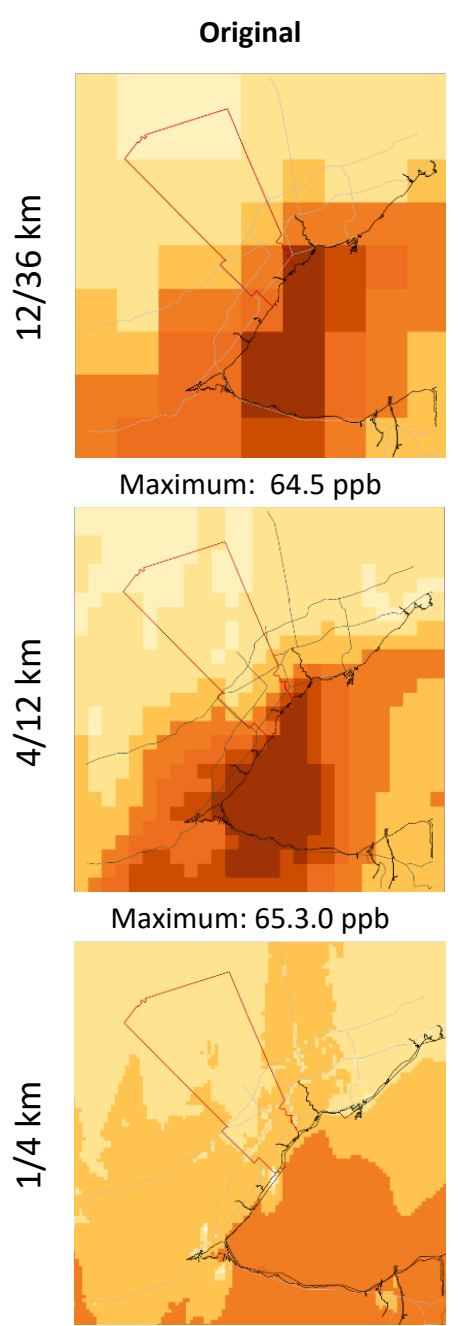

Maximum: $50.3 \mathrm{ppb}$

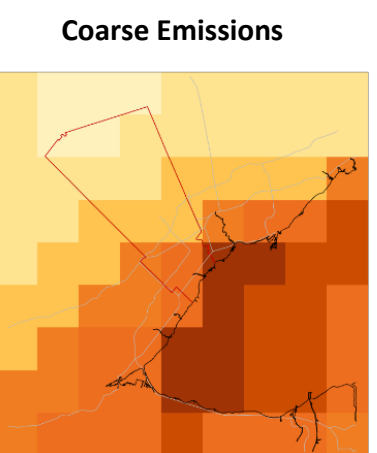

Maximum: $62.0 \mathrm{ppb}$

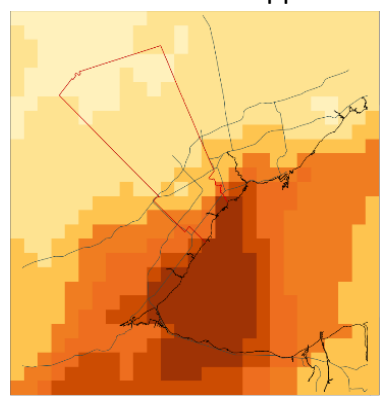

Maximum: $65.1 \mathrm{ppb}$

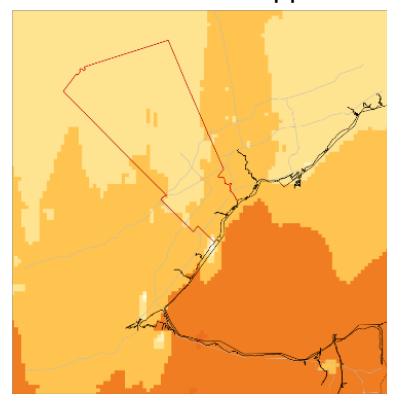

Maximum: $50.2 \mathrm{ppb}$
Coarse Meteorology

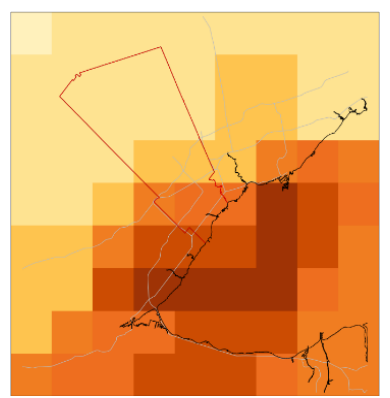

Maximum: $66.5 \mathrm{ppb}$

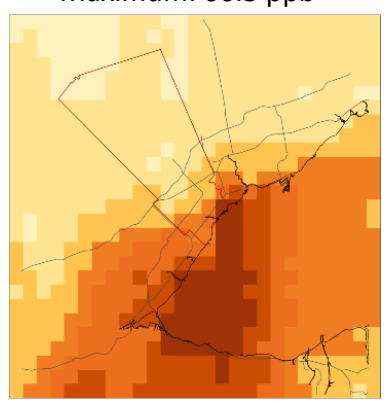

Maximum: $64.8 \mathrm{ppb}$

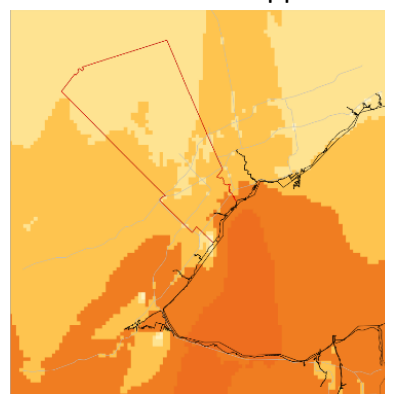

Maximum: $52.2 \mathrm{ppb}$
Coarse Meteorology \& Emissions

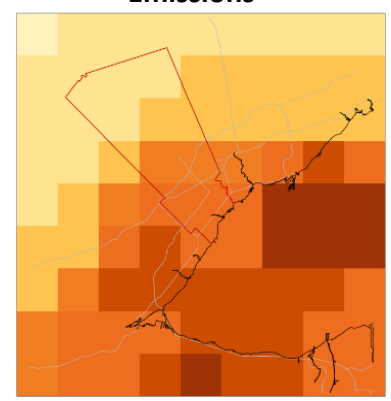

Maximum: $66.5 \mathrm{ppb}$

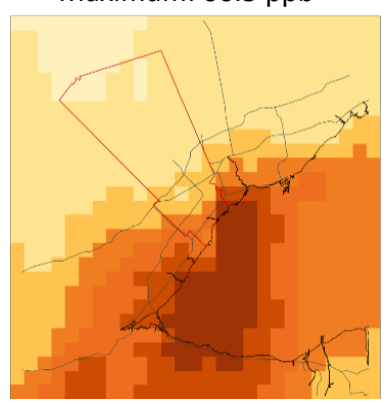

Maximum: 64.9

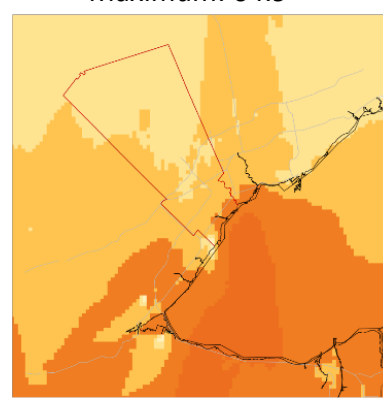

Maximum: $52.1 \mathrm{ppb}$

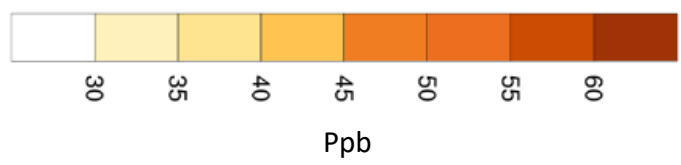

Figure 5-2. Daily Maximum 8-hour average $\mathrm{O}_{3}$ concentration results from various fine and coarse input resolution variation scenarios of 12 and $36 \mathrm{~km}, 4$ and $12 \mathrm{~km}$, and 1 and $4 \mathrm{~km}$ (top to bottom, respectively). 

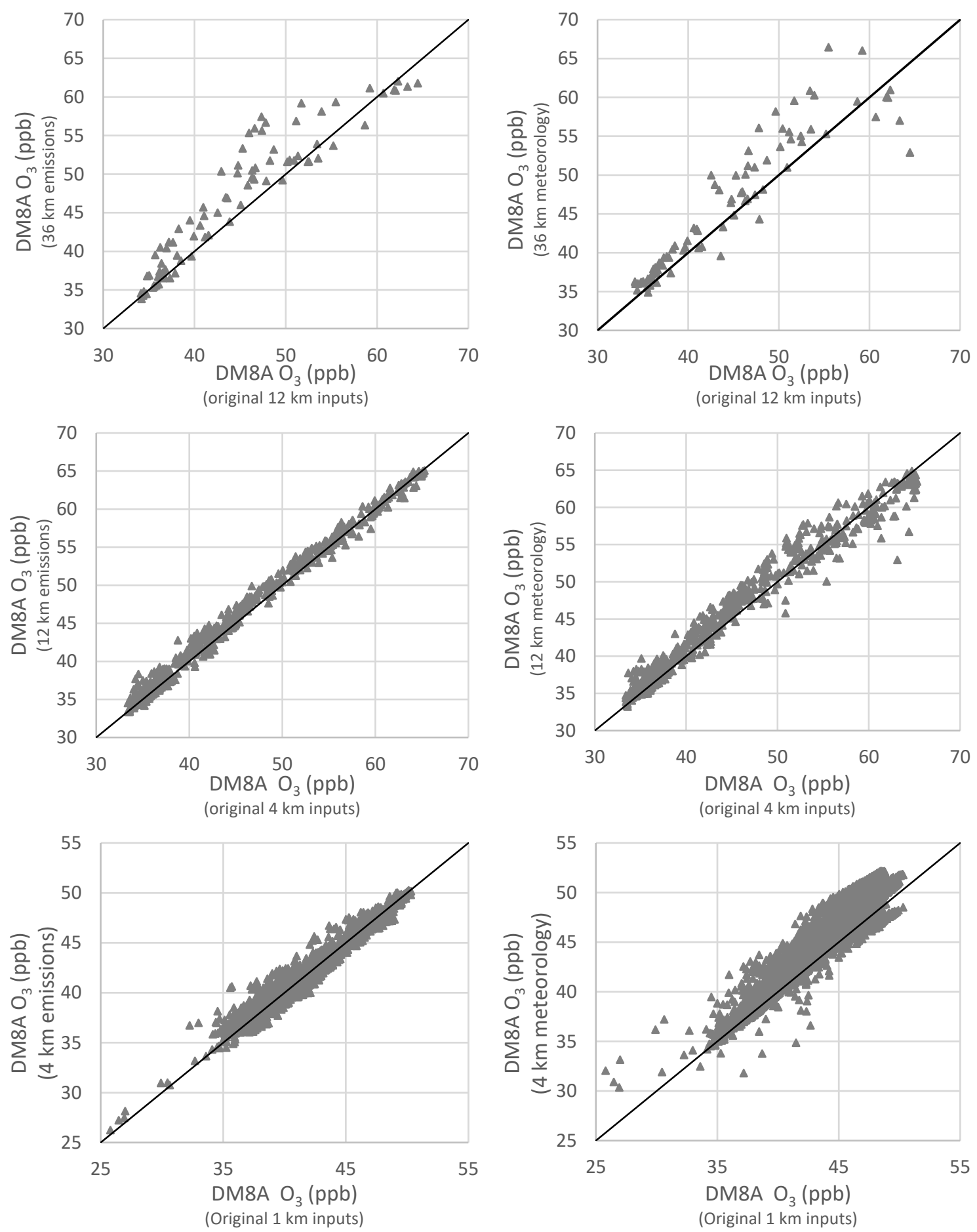

Figure 5-3. Scatter plots comparing the $\mathrm{DM}_{8 \mathrm{~A}} \mathrm{O}_{3}$ all fine resolution concentration results to concentrations generated with coarse emissions (left) and coarse meteorology (right), for resolution variation scenarios of 12 and $36 \mathrm{~km}, 4$ and $12 \mathrm{~km}$, and 1 and $4 \mathrm{~km}$ (top to bottom, respectively). 
The RMSD for $\mathrm{DM} \mathrm{A} \mathrm{O}_{3}$ concentrations for each of the input resolution scenarios (all model inputs and all resolution combinations) are displayed in Table 5-2. These results indicate the average difference in each grid cell due to the change in input resolution. Regardless of the resolution combination, the resolution of the inputted meteorology had a higher impact on results than did the resolution of emissions. The resolution of emissions followed a trend such that the difference increased as the resolution became coarser, e.g. $36 \mathrm{~km}$ emissions difference was greater than the $12 \mathrm{~km}$ emissions difference, which was greater than the $4 \mathrm{~km}$ emissions. This trend does not hold for meteorology, where the 1 and $4 \mathrm{~km}$ scenario was more impacted than the 4 and $12 \mathrm{~km}$ scenario. This suggests that the model performance is very sensitive at fine resolutions to meteorology, where a change between 1 and $4 \mathrm{~km}$ causes a more drastic impact than between 4 and $12 \mathrm{~km}$. Mesoscale meteorology may require a resolution less than 4 $\mathrm{km}$ to accurately simulate.

Table 5-2. RMSD (in ppb) for $\mathrm{DM} \mathrm{A} \mathrm{O}_{3}$ concentrating for each input resolution scenario.

\begin{tabular}{cccc}
\cline { 2 - 4 } Scenario & $\begin{array}{c}\text { RMSD (ppb) } \\
\text { Emissions }\end{array}$ & $\begin{array}{c}\text { Coarse } \\
\text { Meteorology }\end{array}$ & $\begin{array}{c}\text { Coarse } \\
\text { Meteorology } \\
\text { \& Emissions }\end{array}$ \\
\hline $\mathbf{1}$ and $\mathbf{~ k m}$ & 0.38 & 1.59 & 1.61 \\
$\mathbf{4}$ and $12 \mathbf{~ k m}$ & 0.99 & 1.23 & 1.76 \\
$\mathbf{1 2}$ and $\mathbf{3 6} \mathbf{~ k m}$ & 3.22 & 3.76 & 5.90
\end{tabular}

\subsubsection{Nitrogen Dioxide Concentration Results}

Coarse input emissions caused a decrease in the maximum 24-hour average $\mathrm{NO}_{2}$ concentration for the 12 and $36 \mathrm{~km}$, and 4 and $12 \mathrm{~km}$ scenarios (Figures 5-4 and 5-5). Difference plots 
between the concentrations generated at the coarse resolution scenarios and those all-fine resolutions are displayed within the supplementary material, Figures 5-20, 5-22 and 5-24 for the 12 and $36 \mathrm{~km}, 4$ and $12 \mathrm{~km}$, and 1 and $4 \mathrm{~km}$ scenarios, respectively. The maximum 24-hour average $\mathrm{NO}_{2}$ concentrations for all resolution combinations were most impacted by the input resolution of emissions than of meteorology. The concentration of $\mathrm{NO}_{2}$ profoundly relies on the inputted emissions as it is a primary species (i.e. $\mathrm{NO}_{2}$ is directly emitted). The diminishing of maximum $\mathrm{NO}_{2}$ concentrations at the coarser resolutions is consistent with other research findings (Jang et al., 1995; Wu, et al., 2008a). The 1 and $4 \mathrm{~km}$ scenario saw the least impact due to emission resolution, with a slightly low bias for high concentrations, but nothing as notable as the coarser combinations.

High $\mathrm{NO}_{2}$ concentrations within the all-fine $12 \mathrm{~km}$ model occur in urban areas. Since these locations are along the lake-front, averaging of the emissions within a $36 \mathrm{~km}$ grid cell can be between highly urban areas with high emission sources and areas over the water which have relatively low emissions (emissions from ships were considered within the modelling), causing strong spatial gradients. This resulted in high dilution of the emissions within waterfront cells. The location in which the highest difference occurs (most prominent in Figure 5-20) is being diluted over the water. The 4 and $12 \mathrm{~km}$ resolution combination also saw an underestimation of maximum concentrations along the waterfront when emissions were coarse.

As emission maxima are averaged down, surrounding emission minima become averaged up, causing slight overpredictions in subsequent areas. Figure 5-5 demonstrates this, many of the low concentrations at $12 \mathrm{~km}$ input were over-predicted in the $36 \mathrm{~km}$ emission case. Dilution 
over water influences the 12 and $36 \mathrm{~km}$ combinations greater than the other combinations as the $36 \mathrm{~km}$ cells spans a greater area of the water than any 12 or $4 \mathrm{~km}$ cell does.

Concentrations of 24-hour average $\mathrm{NO}_{2}$ were not as heavily influenced by the input resolution of meteorology for the simulation period under analysis as they were by the resolution of emissions for all resolution combinations. The results which had the highest impacts were again those of 12 and 36, in which the $36 \mathrm{~km}$ input spanned both land and water. Parameters, such as windspeed and temperature, alter between water and land, averaging would impact these locations more so than grid cells that consistently over water or consistently land. As previously mentioned, there was no precipitation within the 2-day period of study, which would have a large impact on pollution scavenging. It is predicted that the impacts due to meteorological resolution be a function of mainly transport mixed with temperature impacting loss chemistry. The 4 and $12 \mathrm{~km}$ resolution results saw an increase in maximum concentrations when meteorology was coarse.

The resolution of the CTM had a large influence between the $4 \mathrm{~km}$ and $12 \mathrm{~km}$ models, reducing the maximum drastically, and not capturing the localized high concentrations. The results generated from the $4 \mathrm{~km} \mathrm{CTM}$ were very close to those generated from the $1 \mathrm{~km}$, showing similar magnitudes and trends, however with less spatial detail. It is suggested that $4 \mathrm{~km}$ be a suitable resolution for modelling of $\mathrm{NO}_{2}$. 

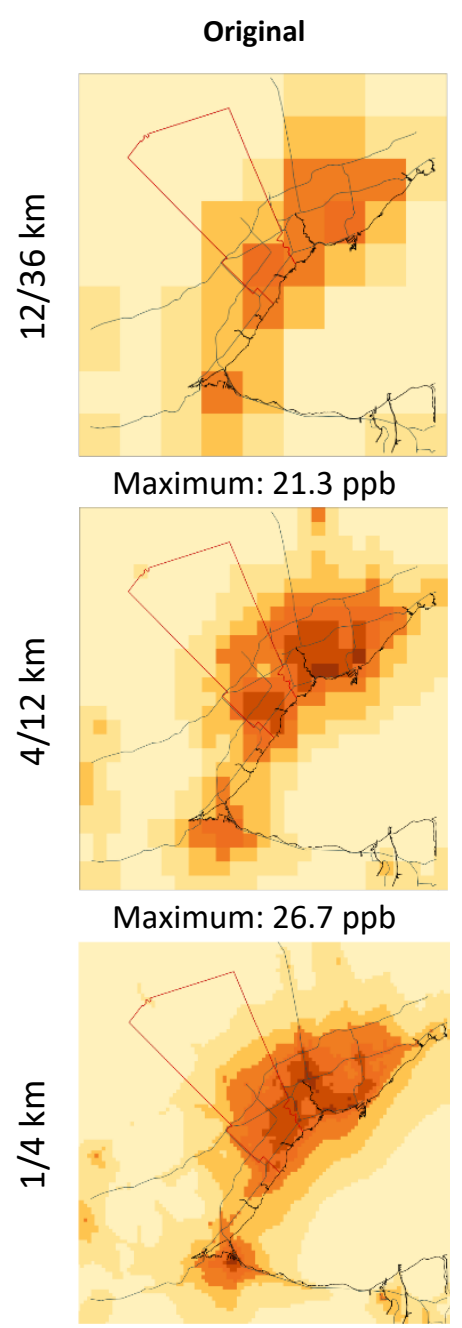

Maximum: 26.8 ppb
Coarse Emissions

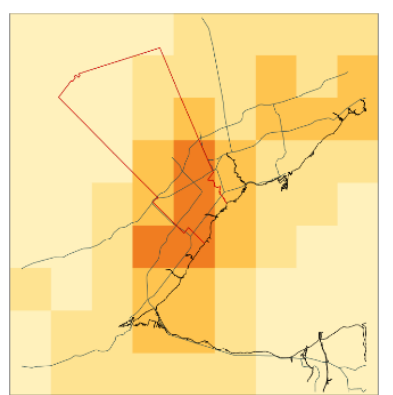

Maximum: $17.0 \mathrm{ppb}$

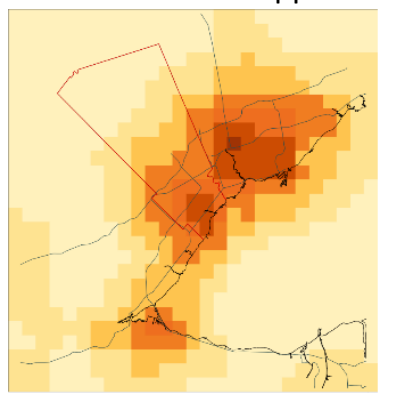

Maximum: $24.2 \mathrm{ppb}$

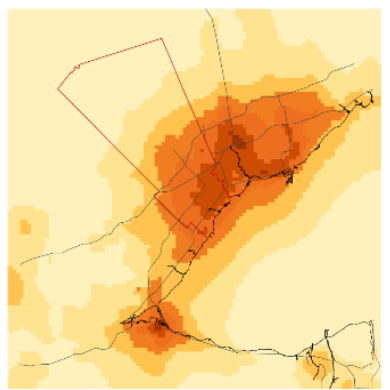

Maximum: $27.2 \mathrm{ppb}$
Coarse Meteorology

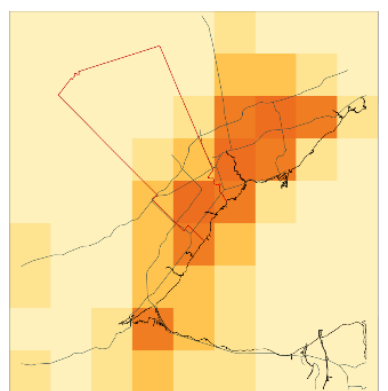

Maximum: $21.2 \mathrm{ppb}$

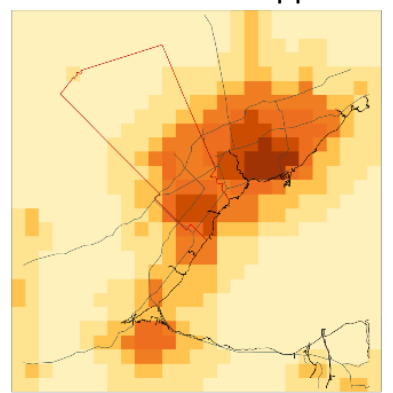

Maximum: 29.8 ppb

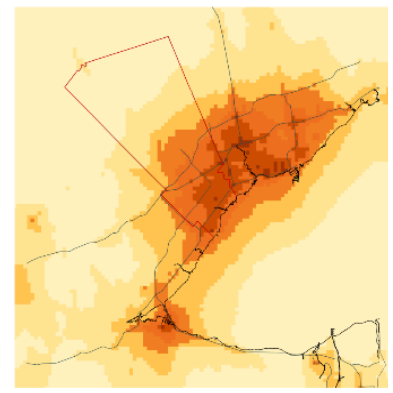

Maximum: 25.9 ppb
Coarse Meteorology \& Emissions

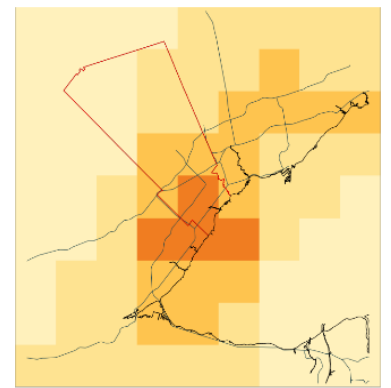

Maximum: $16.2 \mathrm{ppb}$

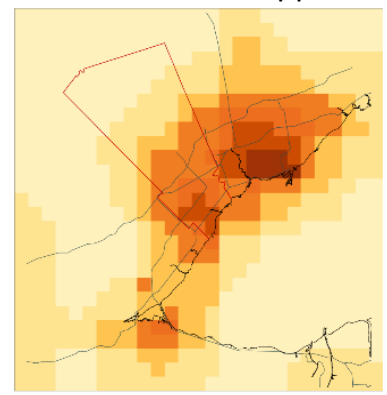

Maximum: $26.2 \mathrm{ppb}$

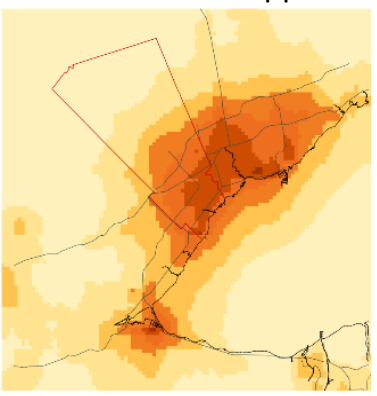

Maximum: 26.9 ppb

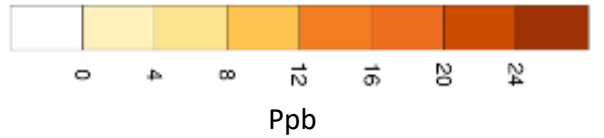

Figure 5-4. 24-hour average $\mathrm{NO}_{2}$ concentration results from various fine and coarse input resolution variation scenarios of 12 and $36 \mathrm{~km}, 4$ and $12 \mathrm{~km}$, and 1 and $4 \mathrm{~km}$ (top to bottom, respectively) 

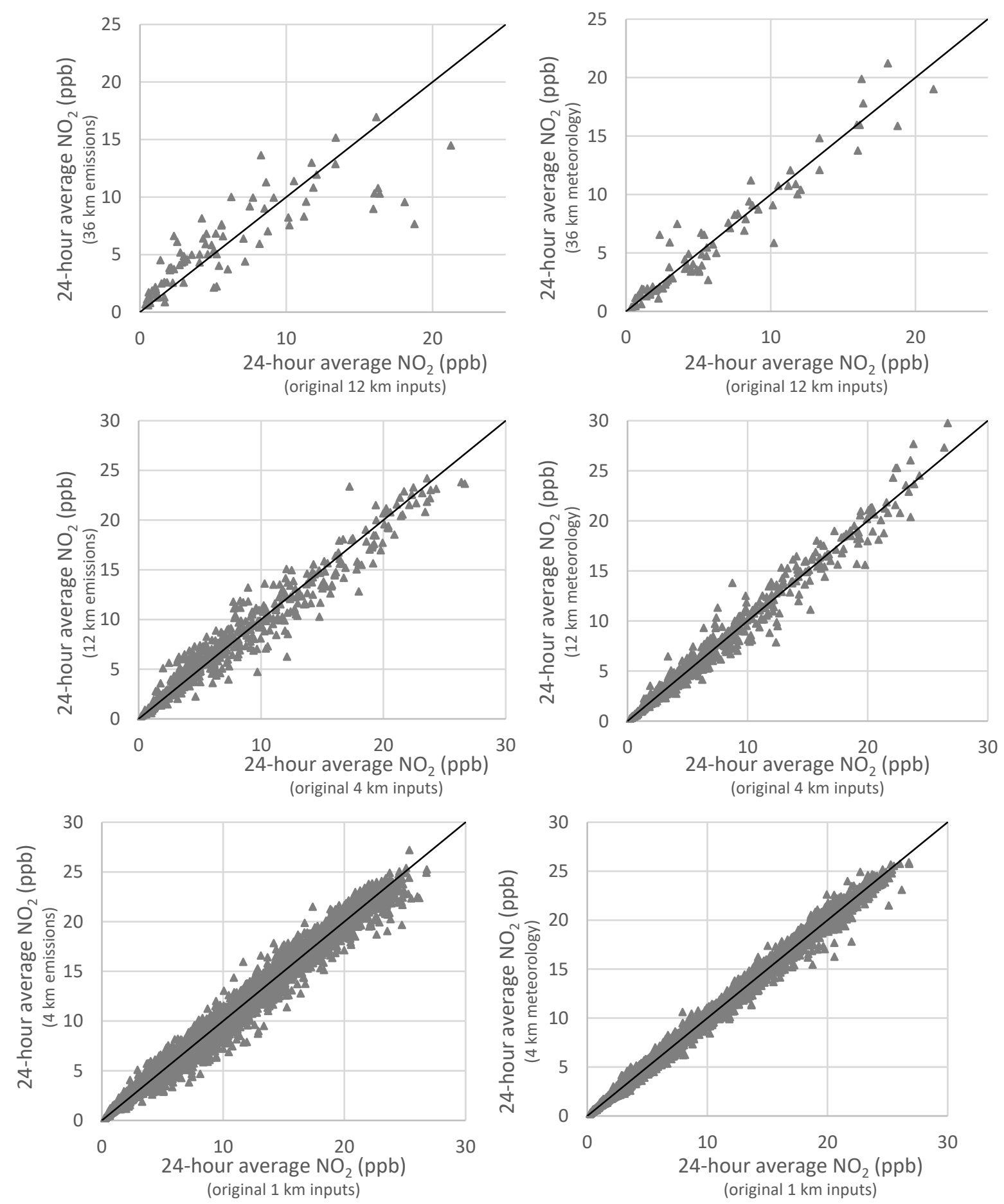

Figure 5-5. Scatter plots comparing the 24-hour average $\mathrm{NO}_{2}$ all fine resolution concentration results to concentrations generated with coarse emissions (left) and coarse meteorology (right), for resolution variation scenarios of 12 and $36 \mathrm{~km}, 4$ and $12 \mathrm{~km}$, and 1 and $4 \mathrm{~km}$ (top to bottom, respectively). 
The RMSD for 24-hour average $\mathrm{NO}_{2}$ concentrations for each of the input resolution scenarios (all model inputs and all resolution combinations) are presented within Table 5-4. Regardless of the resolution combination, the resolution of the inputted emissions had a higher impact on results than did the resolution of meteorology. The highest impact due to input resolution is seen between 12 and $36 \mathrm{~km}$, followed by 4 and $12 \mathrm{~km}$, and lastly the 1 and $4 \mathrm{~km}$ combinations. The relatively small RMSD found with the 1 and $4 \mathrm{~km}$ combinations further suggest that a $4 \mathrm{~km}$ resolution may be sufficient to predict $\mathrm{NO}_{2}$, as this difference is most likely smaller than the error due to uncertainty of the overall model performance.

Table 5-3. RMSD (in ppb) for 24-hour average $\mathrm{NO}_{2}$ concentrations for each input resolution scenario

\begin{tabular}{cccc}
\cline { 2 - 4 } Scenario & $\begin{array}{c}\text { RMSD (ppb) } \\
\text { Emissions }\end{array}$ & $\begin{array}{c}\text { Coarse } \\
\text { Meteorology }\end{array}$ & $\begin{array}{c}\text { Coarse } \\
\text { Meteorology } \\
\text { \& Emissions }\end{array}$ \\
\hline $\mathbf{1}$ and $\mathbf{4} \mathbf{~ k m}$ & 0.52 & 0.37 & 0.64 \\
$\mathbf{4}$ and $12 \mathbf{~ k m}$ & 1.17 & 0.87 & 1.41 \\
$\mathbf{1 2}$ and $\mathbf{3 6} \mathbf{~ k m}$ & 2.82 & 1.41 & 3.09
\end{tabular}

\subsubsection{Fine Particulate Concentration Results}

The locations with the highest 24-hour average $\mathrm{PM}_{2.5}$ concentrations (Figure 5-6 and 5-26, 28 and 30) were drastically underpredicted with coarse emissions for every resolution scenario. The maximum concentration generated from $1 \mathrm{~km}$ emissions was $4 \mathrm{x}$ higher than those from 4 $\mathrm{km}$ emissions, while from $4 \mathrm{~km}$ emissions was $2 x$ higher than those from the $1 \mathrm{~km}$ emissions, demonstrating the extreme dilution occurring near localized maximums over short distances. The location in which the maximum $\mathrm{PM}_{2.5}$ emissions are occurring from is the location of the Toronto Pearson airport. Even emissions being inputted at the relatively fine resolution of $4 \mathrm{~km}$ 
were unable to capture the strong spatial inhomogeneities surrounding the airport (Figure 528). Other areas with high industry (e.g. parts of Hamilton) were also not adequately modelled using $4 \mathrm{~km}$ resolution emissions. Fine particulate matter is composed of numerous species, a combination of primary and secondary species. Due to coagulation of fine particles, they often do not travel far from emission sources, hence high concentrations occur near high emission sources. The large impact of emission resolution on results near these strong emissions sources suggests that the $\mathrm{PM}_{2.5}$ contributing to these discrepancies is most likely primary.

Coarse meteorology also resulted in lower maximum concentrations for every resolution combination. The spatial distribution of differences between the all $12 \mathrm{~km}$ input resolution results and the coarse $36 \mathrm{~km}$ input meteorology for $\mathrm{PM}_{2.5}$ (Figure 5-26) shows similar trends as the respective $\mathrm{NO}_{2}$ did (Figure 5-20). The wind speeds over the lake were higher than over land, and it is suggested that the meteorology resolution related impacts on $\mathrm{PM}_{2.5}$ be a function of transport due to averaging between drastically differing land use parameter, resulting in higher dispersion at the locations of maxima.

The resolution of the CTM did not have a large influence between any resolutions; the results generated from the coarse runs were very close to those generated from the fine runs, showing similar magnitudes and trends. Impacts due to resolution were mainly caused by emissions and it is suggested that areas with strong emission gradients be modelled at a fine resolution. 

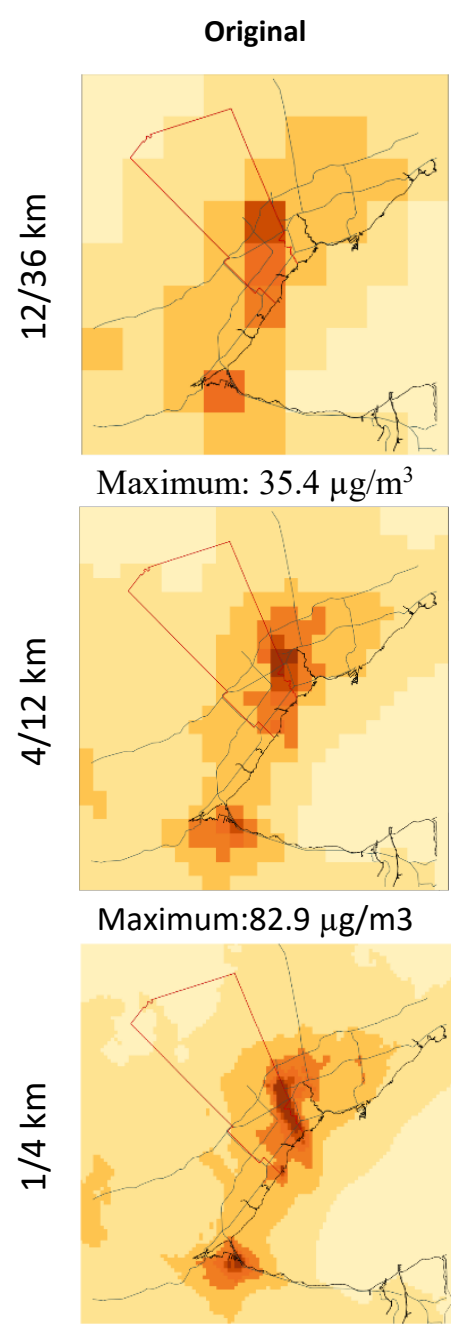

Maximum: $288.9 \mu \mathrm{g} / \mathrm{m}^{3}$
Coarse Emissions

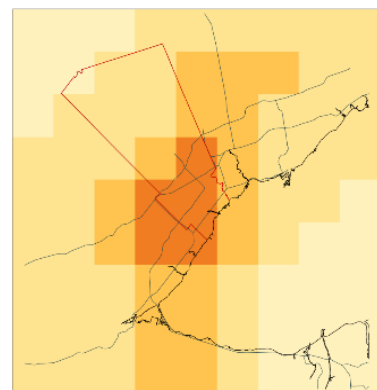

Maximum: $22.8 \mu \mathrm{g} / \mathrm{m}^{3}$

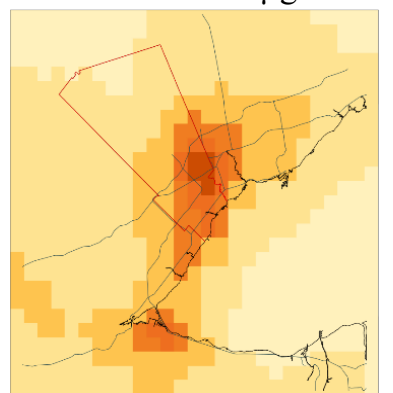

Maximum: $35.2 \mu \mathrm{g} / \mathrm{m} 3$

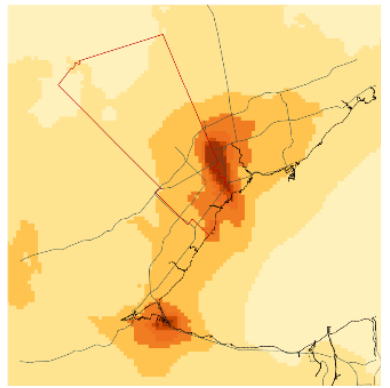

Maximum: $70.1 \mu \mathrm{g} / \mathrm{m}^{3}$
Coarse Meteorology

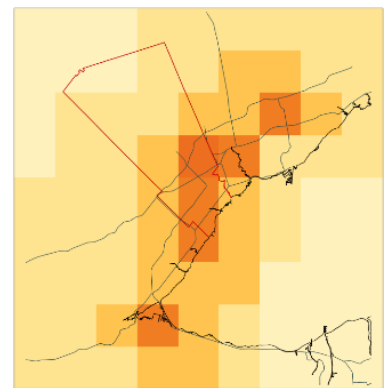

Maximum: $28.4 \mu \mathrm{g} / \mathrm{m}^{3}$

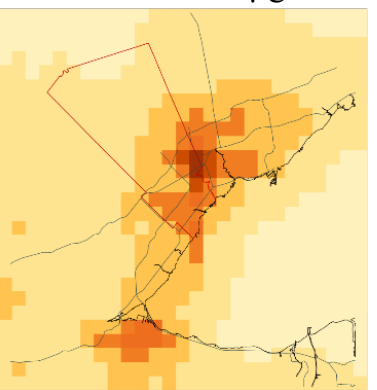

Maximum: $91.9 \mu \mathrm{g} / \mathrm{m} 3$

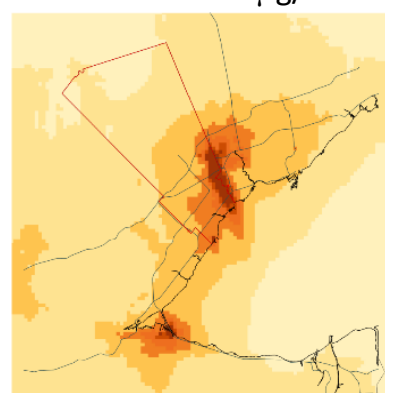

Maximum: $275.0 \mu \mathrm{g} / \mathrm{m}^{3}$
Coarse Meteorology \& Emissions

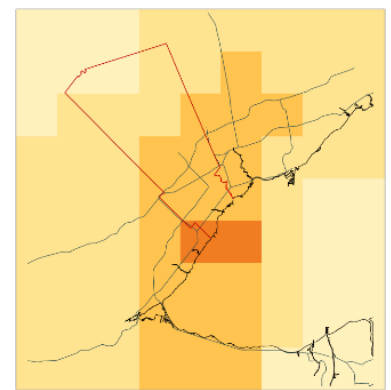

Maximum: $19.3 \mu \mathrm{g} / \mathrm{m}^{3}$

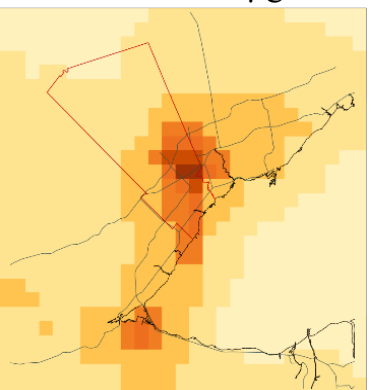

Maximum: $38.5 \mu \mathrm{g} / \mathrm{m} 3$

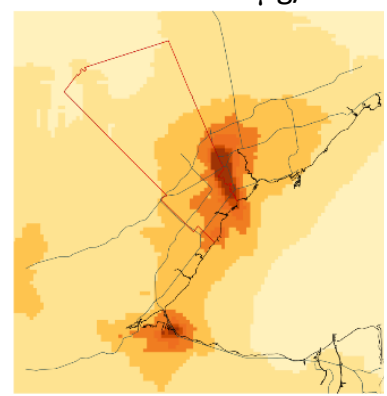

Maximum: $85.7 \mu \mathrm{g} / \mathrm{m}^{3}$

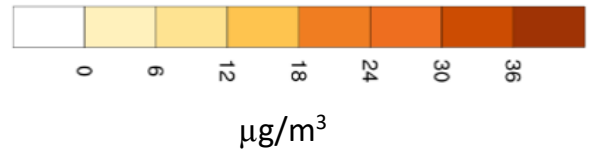

Figure 5-6. 24-hour average $\mathrm{PM}_{2.5}$ concentration results from various fine and coarse input resolution variation scenarios of 12 and $36 \mathrm{~km}, 4$ and $12 \mathrm{~km}$, and 1 and $4 \mathrm{~km}$ (top to bottom, respectively) 

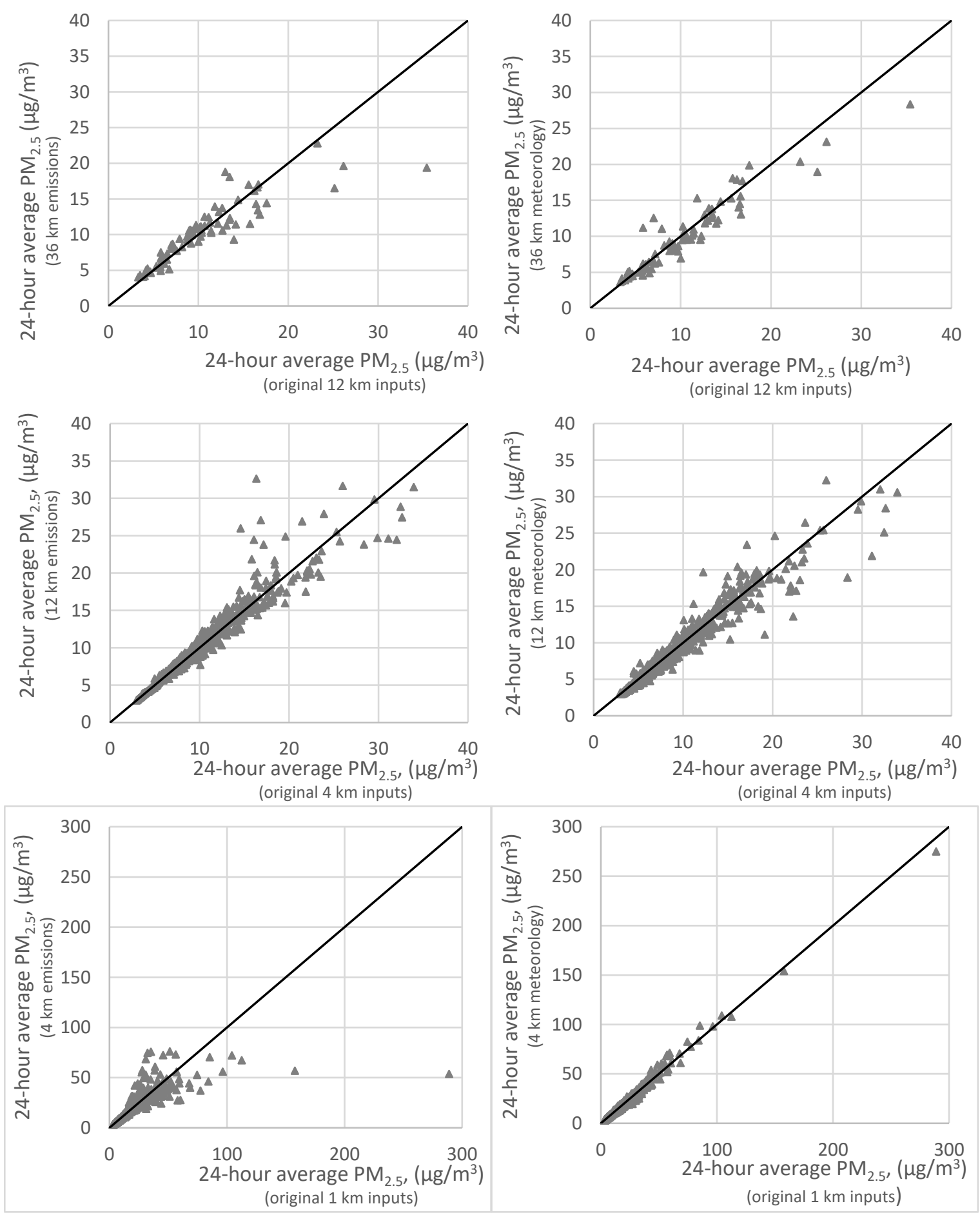

Figure 5-7. Scatter plots comparing the 24-hour average $\mathrm{PM}_{2.5}$ all fine resolution concentration results to concentrations generated with coarse emissions (left) and coarse meteorology (right), for resolution variation scenarios of 12 and $36 \mathrm{~km}, 4$ and $12 \mathrm{~km}$, and 1 and $4 \mathrm{~km}$ (top to bottom, respectively). 
The 24-hour average $\mathrm{PM}_{2.5}$ RMSD results (Table 5-5) were inconsistent between resolution combination, e.g. resolution of emissions did not always have a greater influence than resolution of meteorology for all resolution combinations. The 1 and $4 \mathrm{~km}$ scenario for emissions had a higher RMSD than the 4 and $12 \mathrm{~km}$ or 12 and $36 \mathrm{~km}$ scenarios as this change in resolution saw the most drastic change in the location of maximums (e.g. $76 \%$ decrease in maximum concentration between the all $1 \mathrm{~km}$ resolution inputs and $4 \mathrm{~km}$ resolution emission input, compared to $61 \%$ decrease and $36 \%$ decrease for the 4 and $12 \mathrm{~km}$ combination and 12 and $36 \mathrm{~km}$ combination, respectively). This is due to the very strong spatial gradients occurring over short distances (within $4 \mathrm{~km}$ ).

The maximum 24-hour $\mathrm{PM}_{2.5}$ concentration generated from the original all $1 \mathrm{~km}$ results is 289 $\mu \mathrm{g} / \mathrm{m}^{3}$ while the second highest concentration is drastically lower at $158 \mu \mathrm{g} / \mathrm{m}^{3}$. The cell with the maximum concentration, which covers the area occupied by the Toronto Pearson airport, decreases drastically down to $54 \mu \mathrm{g} / \mathrm{m}^{3}$ when emissions are inputted at $4 \mathrm{~km}$. This value is an extreme outlier, Table 5-5 also displays the RMSD trends with it removed. These results show a more consistent trend, similar to that found by $\mathrm{NO}_{2}$, where the coarser the resolution combination, the higher the differences. Comparing the RMSD between $\mathrm{PM}_{2.5}$ and $\mathrm{NO}_{2}$ (Table 5-5 and Table 5-4), $\mathrm{PM}_{2.5}$ is more heavily influenced by relatively fine resolution changes than $\mathrm{NO}_{2}$ is, as the influence seen between 1 and $4 \mathrm{~km}$ resolution combinations is drastically higher for $\mathrm{PM}_{2.5}$ than it is for $\mathrm{NO}_{2}$. This suggests that $\mathrm{PM}_{2.5}$ be more sensitive to resolution than $\mathrm{NO}_{2}$. The differences seen between 12 and $36 \mathrm{~km}$ resolutions are similar for $\mathrm{PM}_{2.5}$ and $\mathrm{NO}_{2}$. Fine particulate matter being more sensitive to emissions than it is meteorology contradicts the 
findings by Wu et al. (2008a). This may be because the 2-day period in which the analysis was performed did not have any precipitation periods, and Wu et al. (2008a) found that cloud fractions and precipitation was responsible for the higher influence due to meteorology.

Table 5-4. RMSD for 24-hour average $\mathrm{PM}_{2.5}$ concentrating for each input resolution scenario for all results (top) and with the extreme outlier removed (bottom)

\begin{tabular}{ccccc}
\cline { 3 - 5 } & Scenario & $\begin{array}{c}\text { RMSD (ppb) } \\
\text { Emissions }\end{array}$ & $\begin{array}{c}\text { Coarse } \\
\text { Meteorology }\end{array}$ & $\begin{array}{c}\text { Coarse } \\
\text { Meteorology } \\
\text { \& Emissions }\end{array}$ \\
\cline { 3 - 5 } & $\mathbf{1}$ and $\mathbf{4} \mathbf{~ k m}$ & 2.91 & 0.70 & 2.95 \\
All Results & $\mathbf{4}$ and $\mathbf{1 2} \mathbf{~ k m}$ & 2.45 & 1.33 & 2.82 \\
& $\mathbf{1 2}$ and $\mathbf{3 6} \mathbf{~ k m}$ & 2.69 & 1.89 & 3.58 \\
\hline Extreme & $\mathbf{1}$ and $\mathbf{4} \mathbf{~ k m}$ & 1.68 & 0.69 & 1.78 \\
Outlier & $\mathbf{4}$ and $\mathbf{1 2} \mathbf{~ k m}$ & 2.45 & 1.33 & 2.82 \\
Removed & $\mathbf{1 2}$ and $\mathbf{3 6} \mathbf{~ k m}$ & 2.69 & 1.89 & 3.58
\end{tabular}

It is suggested from the results found in this study that when modeling $\mathrm{PM}_{2.5}$, a resolution of 4 $\mathrm{km}$ or less is required, and for areas with very strong emission spatial gradients, due to airports or possibly power plants, a resolution of $4 \mathrm{~km}$ may not adequately model the high concentrations. Other studies have found that even a resolution of $4 \mathrm{~km}$ might not be fine enough to accurately predict PM concentrations (Mensink et al., 2008; Ott et al., 2008, Shreshtha et al., 2009; Tan et al., 2015).

\subsubsection{Resolution Impact Comparisons between Species}

Nitrogen dioxide and $\mathrm{PM}_{2.5}$ saw similar responses to the resolution of inputs. Both species were more sensitive to the resolution of emissions over meteorology and both species saw an underestimation in concentration when coarse emissions or when coarse meteorology was 
used. Ozone had different responses than $\mathrm{PM}_{2.5}$ and $\mathrm{NO}_{2}$ to resolution, as it was more sensitive to the resolution of meteorology than it was emissions.

Interestingly, coarse emissions in some cases increased the $\mathrm{O}_{3}$ maximum concentrations. Ozone is a secondary pollutant, with a relatively long tropospheric lifetime of 23 days (in the Northern hemisphere), while $\mathrm{NO}_{2}$ is a primary pollutant with a shorter lifetime ranging from a couple of hours to days, and $\mathrm{PM}_{2.5}$ is composed of different species, some primary and some secondary, with atmospheric lifetimes that range from hours to weeks (Seinfeld \& Pandis, 2006). Species that have shorter atmospheric lifetimes have shorter dispersal ranges and tend to have higher concentrations near their source, causing them to be more sensitive to the influences of grid resolution (U.S. EPA, 2007; Punger \& West, 2013). Relative to the magnitude of concentration predictions, $\mathrm{O}_{3}$ was least impacted by input resolutions than $\mathrm{NO}_{2}$ and $\mathrm{PM}_{2.5}$.

Fine particulate matter had a very high RMSD between 1 and $4 \mathrm{~km}$ emissions, as there were a few outlier cells within the domain with extremely high emission rates (those surrounding the airport) which even at the relatively fine resolution of $4 \mathrm{~km}$ substantial dilution occurred. Ott et al. (2008) found that PM concentrations were heterogeneous over short distances by monitoring PM at a series of stations with an average of $4.4 \mathrm{~km}$ between sites. Around areas with strong spatial emission gradients, such as airports, it suggests that $\mathrm{PM}_{2.5}$ is more sensitive to the resolution of emissions than $\mathrm{NO}_{2}$ due to its strong heterogeneity of emissions. When these outliers are removed, the RMSD, relative to the concentration predictions, show that $\mathrm{NO}_{2}$ may be more sensitive to resolution. 
The resolution of the CTM had the greatest impact on $\mathrm{O}_{3}$ between resolutions of $1 \mathrm{~km}$ and 4 $\mathrm{km}$, in which the $1 \mathrm{~km}$ results were notably lower. When the resolution of the CTM was $12 \mathrm{~km}$, maxima were decreased compared to those of the $4 \mathrm{~km}$. The CTM did a good job in capturing concentration magnitudes and trends between all resolutions of $\mathrm{PM}_{2.5}$.

\subsubsection{Exposure Health Impact Results}

Chronic health impacts, parameterized by annual monetized mortality due to long-term exposure to the concentrations presented within Section 5.3.1, were estimated for each resolution scenario. To keep the presentation of results concise and to only focus on the most prominent impacts, figures and result comparisons were generated for only the impacts of resolution on each parameter individually, e.g. impacts due to the resolution of meteorology, emissions, and population individually, opposed to showing meteorology paired with emissions, or other pairings.

The health impact results generated at different resolution scenarios were compared with difference figures, root mean squared difference (RMSD) values, and scatter comparison plots. Additionally, the total domain-wide health impacts were estimated by summing the health impacts in each grid cell over the domain, allowing for direct comparisons between different resolutions.

As demonstrated by equation 1 , health impacts are exponentially influenced by concentration. As such, a small change in a concentration may result in a substantial impact on the estimated 
related health impacts, especially in locations of high population (high exposure). Also demonstrated by equation 1 , health impacts are directly proportional to population inputs.

\subsubsection{Ozone Related Health Impact Results}

The chronic health impacts, parameterized by monetized mortality, due to chronic exposure to the $\mathrm{O}_{3}$ concentrations presented within Section 5.3.1 are displayed in Figure 5-8 for each input parameter and for each resolution combination. The maximum health impacts due to $\mathrm{O}_{3}$ exposure increased when emissions were coarse for every resolution combination. This is inconsistent with that was found in terms of concentration, in which the maximum $D M 8 \mathrm{~A} \mathrm{O}_{3}$ decreased with coarse emissions. Health impacts are also a function of population, and the maximum $\mathrm{O}_{3}$ concentration occurs over the water in which there is no exposure, explaining the inconsistency in the trends.

The grid cell in which the maximum increase in health impacts occurs for the $12 \mathrm{~km}$ case did see a slight concentration increase with coarse $(36 \mathrm{~km})$ emissions. The high population in this grid cell causes the intensity of the health impacts to be magnified. Ozone being a secondary pollutant has its maximum downwind of precursor emission sources. Using coarse emission data causes the emissions to be spread uniformly over a larger area, which can inaccurately cause false downwind locations in which $\mathrm{O}_{3}$ forms at, such as the downtown area which had the highest increase in maximum health impacts. Another reason this location may have increased could be due to the dilution of NOx in slight NOx-inhibited areas, decreasing the NO-titration 
that reduces $\mathrm{O}_{3}$. All locations with the highest health impacts were increased (Figures 5-8) when emissions were coarse.

Coarse meteorology caused slight decreases in the maximum health impacts for the 12 and 36, and 4 and $12 \mathrm{~km}$ resolution combinations, while the 1 and $4 \mathrm{~km}$ saw a slight increase. As emissions and meteorology did not impact the health impact results as drastically as population, the scatter comparison plots for these parameters were not presented within the paper. They can be found in the supplementary material within Figures 5-33, 5-35 and 5-37.

The resolution of population had the greatest influence on health impact predictions for all resolutions (Figures 5-8 and 5-9). The areas in which the highest population densities occur is near the water front (downtown Toronto). Many of the cells near the water front are averaged with cells over the water, in which there is no population. This results in a significant dilution of the population on land, and a false representation of population over water. Using coarse resolution population almost consistently underpredicted locations with high health estimates (Figure 5-9) for all resolutions. 

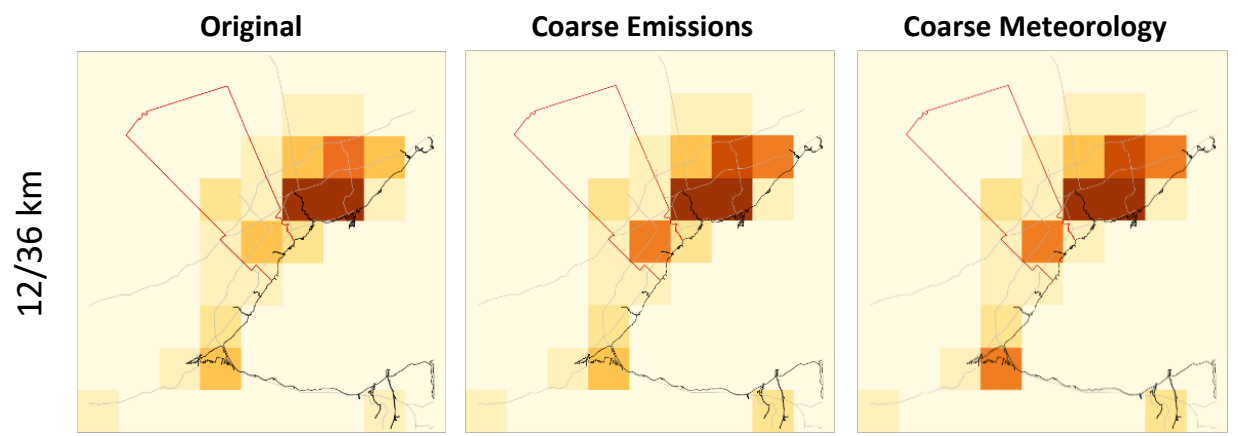

Coarse Population

Maximum: \$5.78 B

Maximum: $\$ 6.17 \mathrm{~B}$

Maximum: \$5.76B

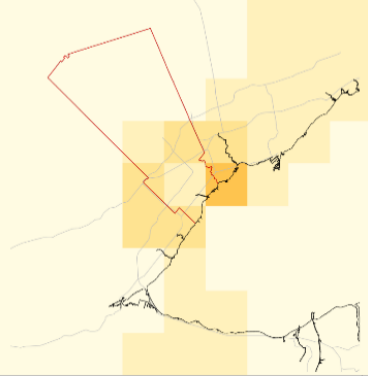

Maximum: \$1.83B

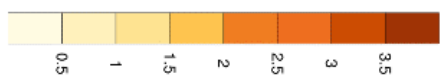

$\$ 10^{9} /$ year

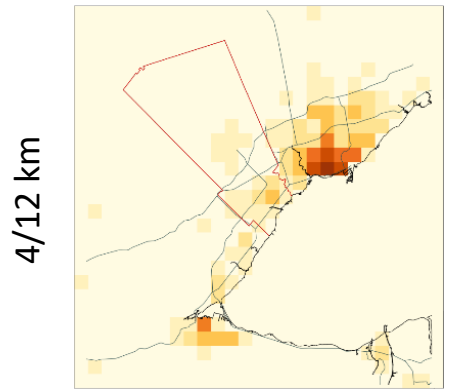

Maximum: \$1.11B
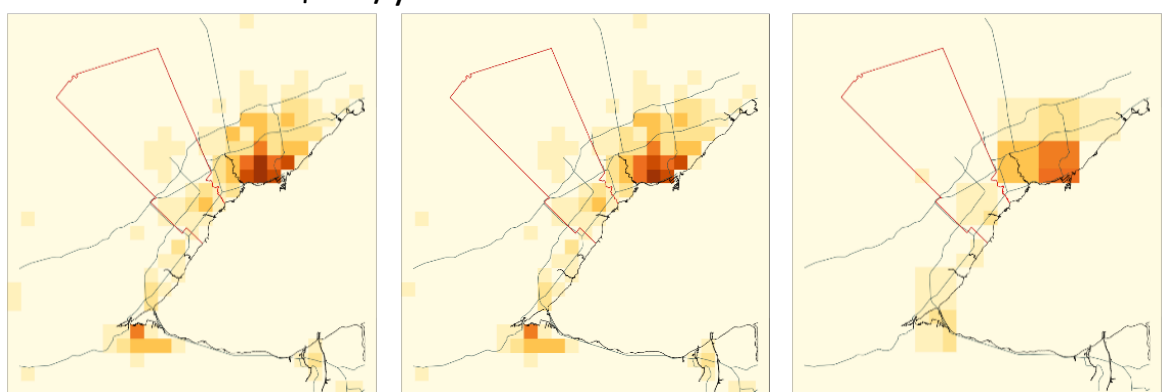

Maximum: \$1.18B

Maximum: \$1.09B

Maximum: \$0.64B
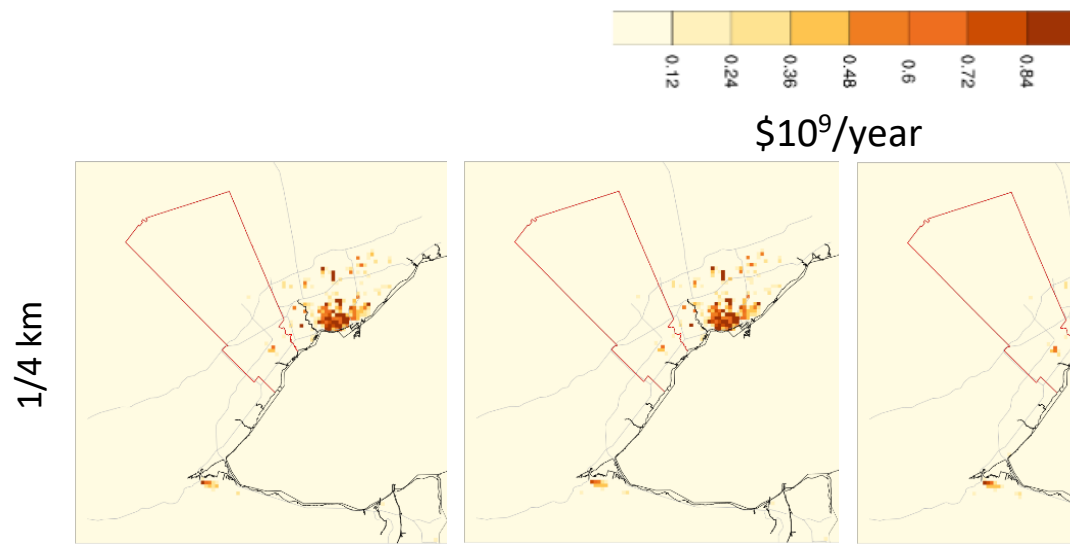

Maximum: \$159.2M
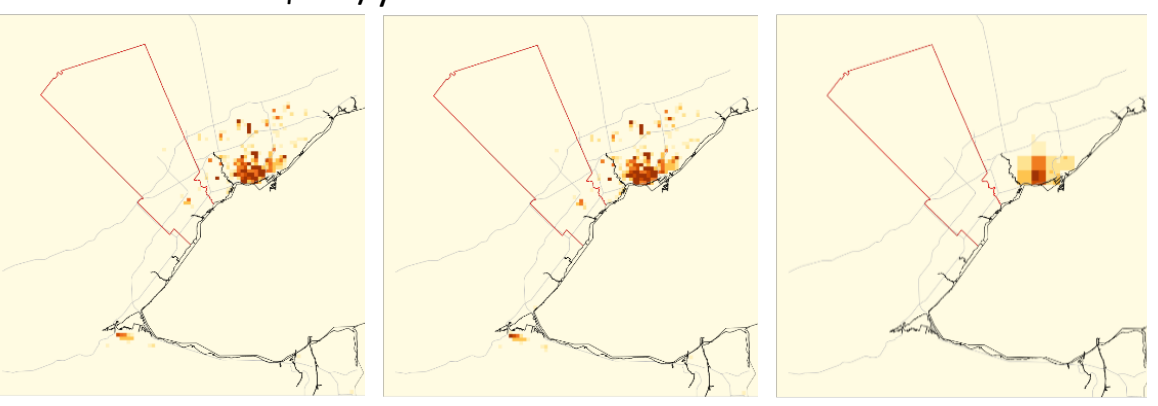

Maximum: \$164.9M Maximum: \$165.7M

Maximum: $\$ 61.6 \mathrm{M}$

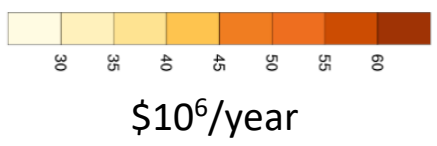

Figure 5-8 Monetized annual health impacts due to Chronic $\mathrm{O}_{3}$ exposure from various fine and coarse input resolution variation scenarios of 12 and $36 \mathrm{~km}, 4$ and $12 \mathrm{~km}$, and 1 and $4 \mathrm{~km}$ (top to bottom, respectively) 

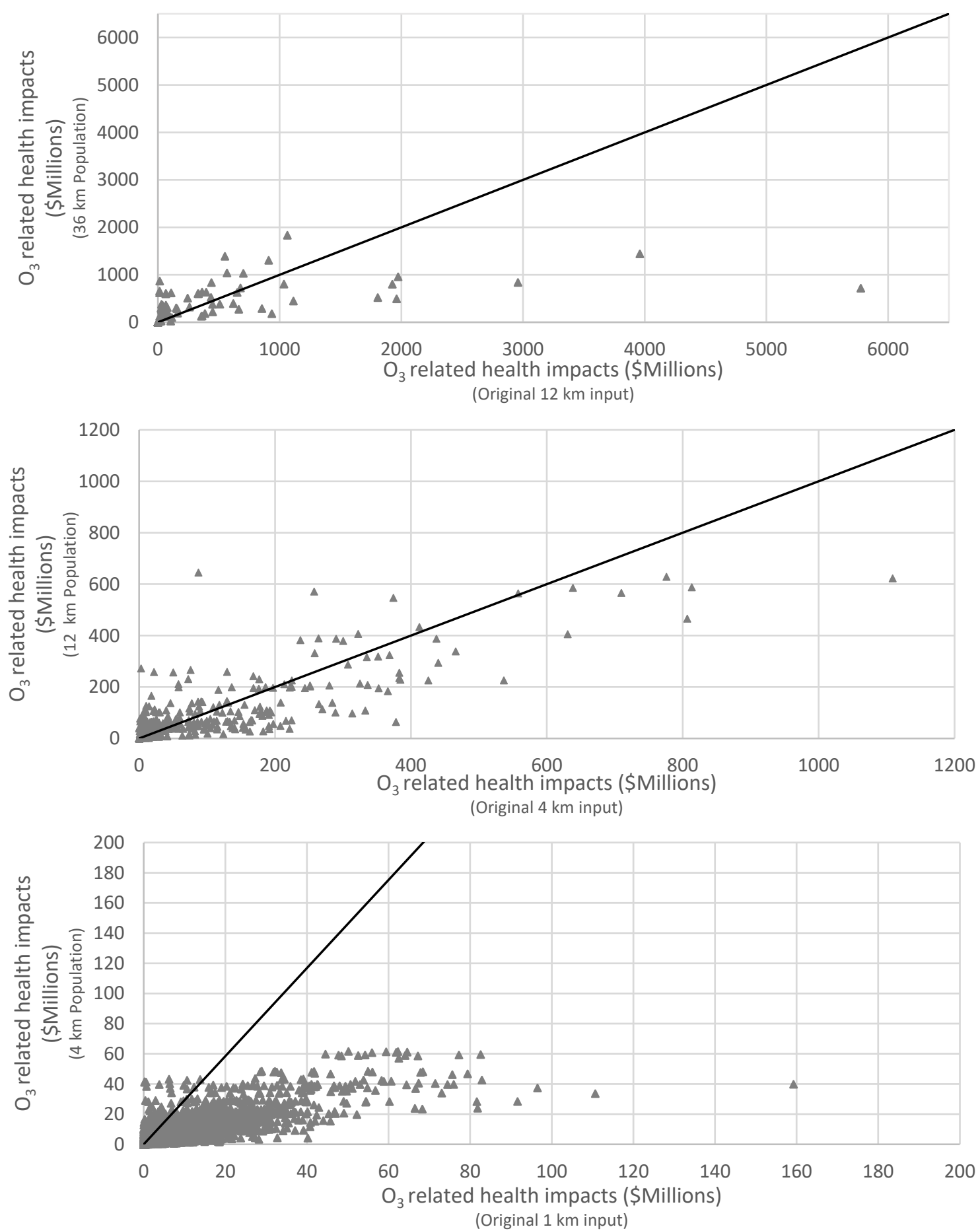

Figure 5-9. Scatter plots comparing the monetized annual health impacts due to chronic $\mathrm{O}_{3}$ exposure results generated at all fine resolution with coarse population, for resolution variation scenarios of 12 and $36 \mathrm{~km}, 4$ and 12 $\mathrm{km}$, and 1 and $4 \mathrm{~km}$ (top to bottom, respectively). 
The domain-wide total annual health impacts due to chronic $\mathrm{O}_{3}$ exposure are displayed in Table 5-6 for all resolution combinations. The most drastic domain-wide impacts were seen between 12 and $36 \mathrm{~km}$ resolution combinations, specifically the $28 \%$ decrease in total domain health impacts from $36 \mathrm{~km}$ population (\$26.0 billion) compared to $12 \mathrm{~km}$ population ( $\$ 35.9$ billion). Coarse emissions and coarse meteorology increased the total monetized health impacts due to $\mathrm{O}_{3}$ exposure for every resolution combination, while population consistently decreased it. The lower decrease due to coarse population between 1 and $4 \mathrm{~km}$, or between 4 and $12 \mathrm{~km}$ would be due to the less drastic spatial gradients seen over shorter distances, and the smaller area over the water being averaged with.

As the resolution becomes coarser, the total domain-wide impacts due to $\mathrm{O}_{3}$ exposure became higher; the all fine $1 \mathrm{~km}$ resolution results were lower than the all $4 \mathrm{~km}$, which were lower than the all $12 \mathrm{~km}$ resolution results. This is in agreeance with other studies which have also demonstrated that as the resolution becomes coarser, domain wide health impacts are overpredicted (Thompson \& Selin, 2012; Punger \& West, 2013). The 1 km resolution concentration results were also lower than the other resolutions, which contributes to the lower health impacts. 
Table 5-5. Domain-wide total annual health impacts due to exposure to $\mathrm{O}_{3}$

\begin{tabular}{ccccc}
\cline { 2 - 5 } Scenario & \multicolumn{3}{c}{ Domain-wide total annual monetized health impacts $(\mathbf{\$ 1 0} \mathbf{)})$} \\
\cline { 2 - 5 } & $\begin{array}{c}\text { Original } \\
\text { (Fine) }\end{array}$ & $\begin{array}{c}\text { Coarse } \\
\text { Emissions }\end{array}$ & $\begin{array}{c}\text { Coarse } \\
\text { Meteorology }\end{array}$ & $\begin{array}{c}\text { Coarse } \\
\text { Population }\end{array}$ \\
\hline $\mathbf{1 2}$ and $\mathbf{3 6} \mathbf{~ k m}$ & 35.9 & 37.5 & 36.7 & 26.0 \\
$\mathbf{4}$ and $\mathbf{1 2} \mathbf{~ k m}$ & 35.7 & 36.5 & 35.7 & 34.3 \\
$\mathbf{1}$ and $\mathbf{4} \mathbf{~ k m}$ & 33.5 & 33.7 & 34.7 & 33.1
\end{tabular}

Table 5-7 displays the monetized annual health impacts due to chronic $\mathrm{O}_{3}$ exposure RMSD results. The drastic difference in magnitude between resolution combinations is because health impacts are an extensive (i.e. scales with the area) parameter.

The resolution of population had the largest impact on all resolution combinations. For the 1 and $4 \mathrm{~km}$ and the 4 and $12 \mathrm{~km}$ combinations, meteorology had a larger influence than emissions, however, the 12 and $36 \mathrm{~km}$ resolution combinations had almost equal error due to emissions as meteorology. RMSD does not give any indication of the sign of error, referring to Figures 5-9, 5-32, 5-34 and 5-36, it is found that coarse emissions increased health impacts whereas meteorology decreased them. The $36 \mathrm{~km}$ case being almost equally as sensitive to emissions as meteorology can be explained by where the impacts on concentration occur and where population densities are the highest. Meteorology still has a higher influence on concentrations, however, these concentrations occur downwind where the populations are not as high (or in this case, are zero over the water). 
Table 5-6. RMSD for the monetized health impacts due to $\mathrm{O}_{3}$ exposure for each input resolution scenario

\begin{tabular}{cccc}
\cline { 2 - 4 } Scenario & \multicolumn{3}{c}{ RMSD (\$) } \\
\cline { 2 - 4 } & $\begin{array}{c}\text { Coarse } \\
\text { Emissions }\end{array}$ & $\begin{array}{c}\text { Coarse } \\
\text { Meteorology }\end{array}$ & $\begin{array}{c}\text { Coarse } \\
\text { Population }\end{array}$ \\
\hline $\mathbf{1}$ and $4 \mathbf{~ k m}$ & $1.68 \times 10^{5}$ & $4.01 \times 10^{5}$ & $4.22 \times 10^{6}$ \\
$\mathbf{4}$ and $12 \mathbf{~ k m}$ & $4.42 \times 10^{6}$ & $4.82 \times 10^{6}$ & $6.21 \times 10^{7}$ \\
$\mathbf{1 2}$ and $36 \mathbf{~ k m}$ & $6.04 \times 10^{7}$ & $5.39 \times 10^{7}$ & $7.83 \times 10^{8}$
\end{tabular}

\subsubsection{Nitrogen Dioxide Health Impact Results}

Coarse emissions caused a decrease in maxima $\mathrm{NO}_{2}$ related health impacts for every resolution, with the largest impact between 12 and $36 \mathrm{~km}$. The change in maximum 24-hour $\mathrm{NO}_{2}$ concentration when using $36 \mathrm{~km}$ resolution emissions only decreased by $20 \%$ from $12 \mathrm{~km}$ resolution emissions, however, when related to associated health impacts, the maximum health impacts in a single $12 \times 12 \mathrm{~km}$ grid cell decreases a substantial $58 \%$ from $\$ 4.96$ billion to $\$ 2.09$ billion. This is attributable to the fact that health impacts are exponentially influenced by concentration (refer to equation 1). As such, a small change in a concentration may result in a substantial impact on the estimated related health impacts. The locations that had the highest impacts due to emission resolution are often urban areas with high populations, further magnifying the impacts on health impacts. Most of the impacts due to resolution of emissions cause an underprediction in health estimates, which is a consistent trend for all the highest estimates (Figures 5-16 and 5-17).

The resolution of meteorology did not have as strong of an influence on health impacts as it did on concentrations, which may be because many of the impacts on concentration occurred over the water which has no population and no exposure. 
The resolution of population had the greatest influence on health impact predictions as evident by Figures 5-10 and 5-11. As described for $\mathrm{O}_{3}$, this is a results of the dilution between the highest populated locations (along the waterfront) and the lowest populated locations over the water which has zero population. This results in a significant dilution of the population on land, and a false representation of population over water. Using coarse resolution population consistently underpredicted health estimates for every resolution. 


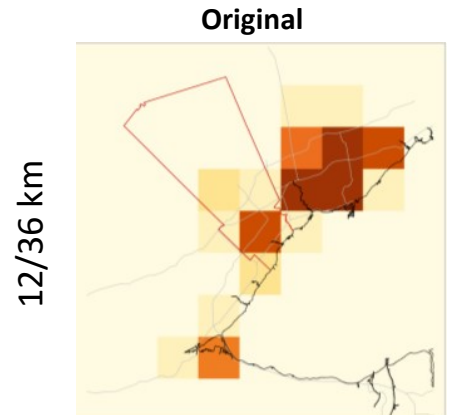

Maximum: \$4.96B

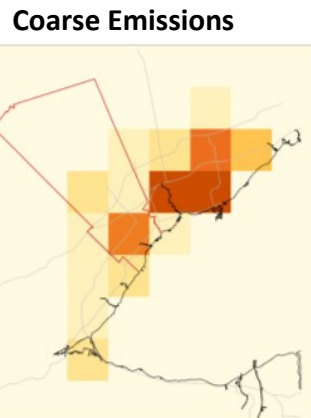

Maximum: \$2.09B

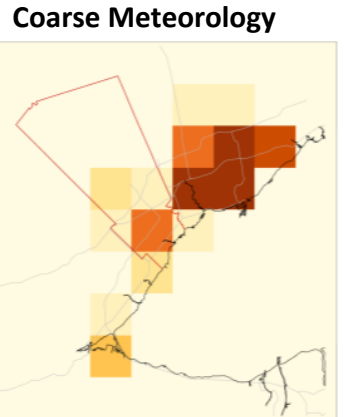

Maximum: \$4.22B
Coarse Population

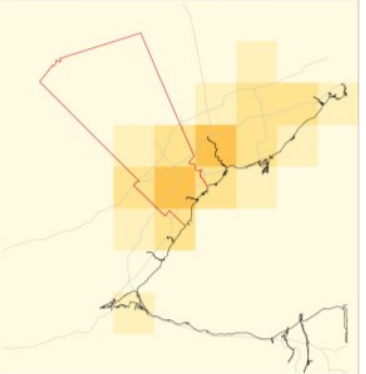

Maximum: \$1.02B

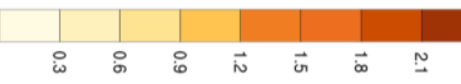

$\$ 10^{9} /$ year

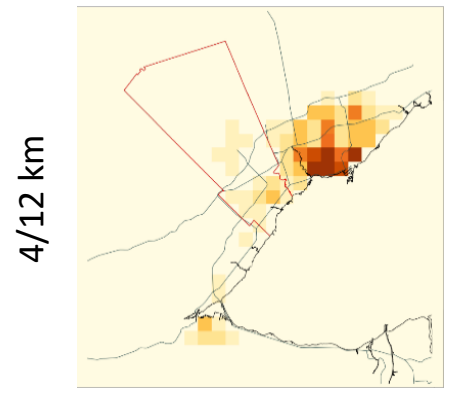

Maximum: \$1.36B

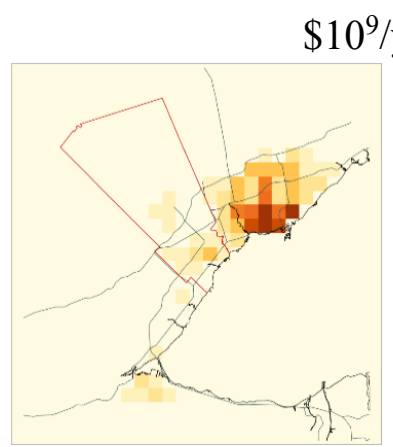

Maximum: \$1.22

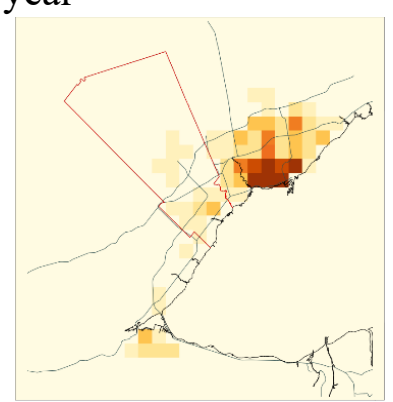

Maximum: \$1.51B

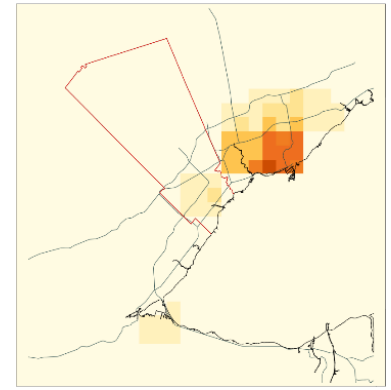

Maximum: \$0.76B

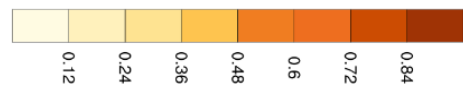

$\$ 10^{9} /$ year

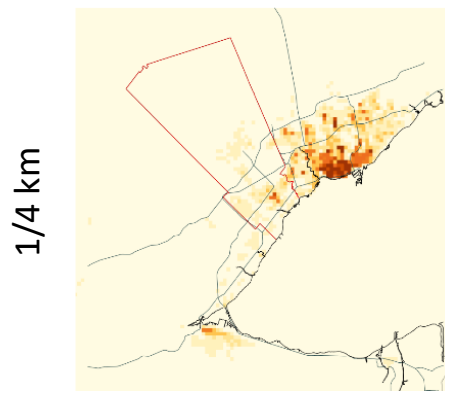

Maximum: \$220.1M

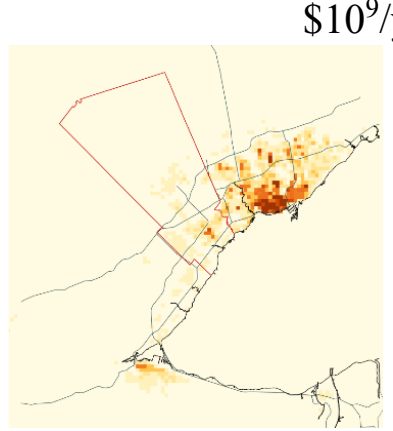

Maximum: \$196.9M

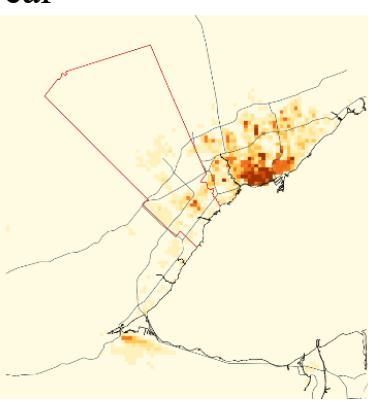

Maximum: \$220.9M

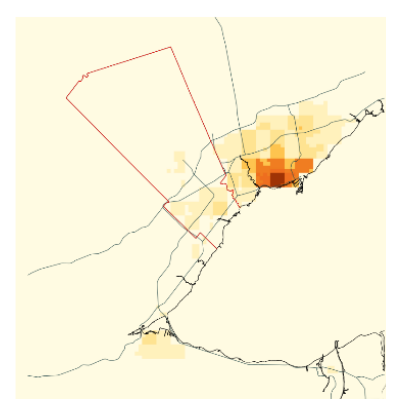

Maximum: \$79.0M

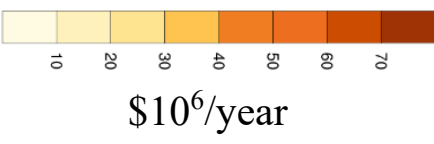

Figure 5-10. Monetized annual health impacts due to Chronic $\mathrm{NO}_{2}$ exposure from various fine and coarse input resolution variation scenarios of 12 and $36 \mathrm{~km}, 4$ and $12 \mathrm{~km}$, and 1 and $4 \mathrm{~km}$ (top to bottom, respectively). 

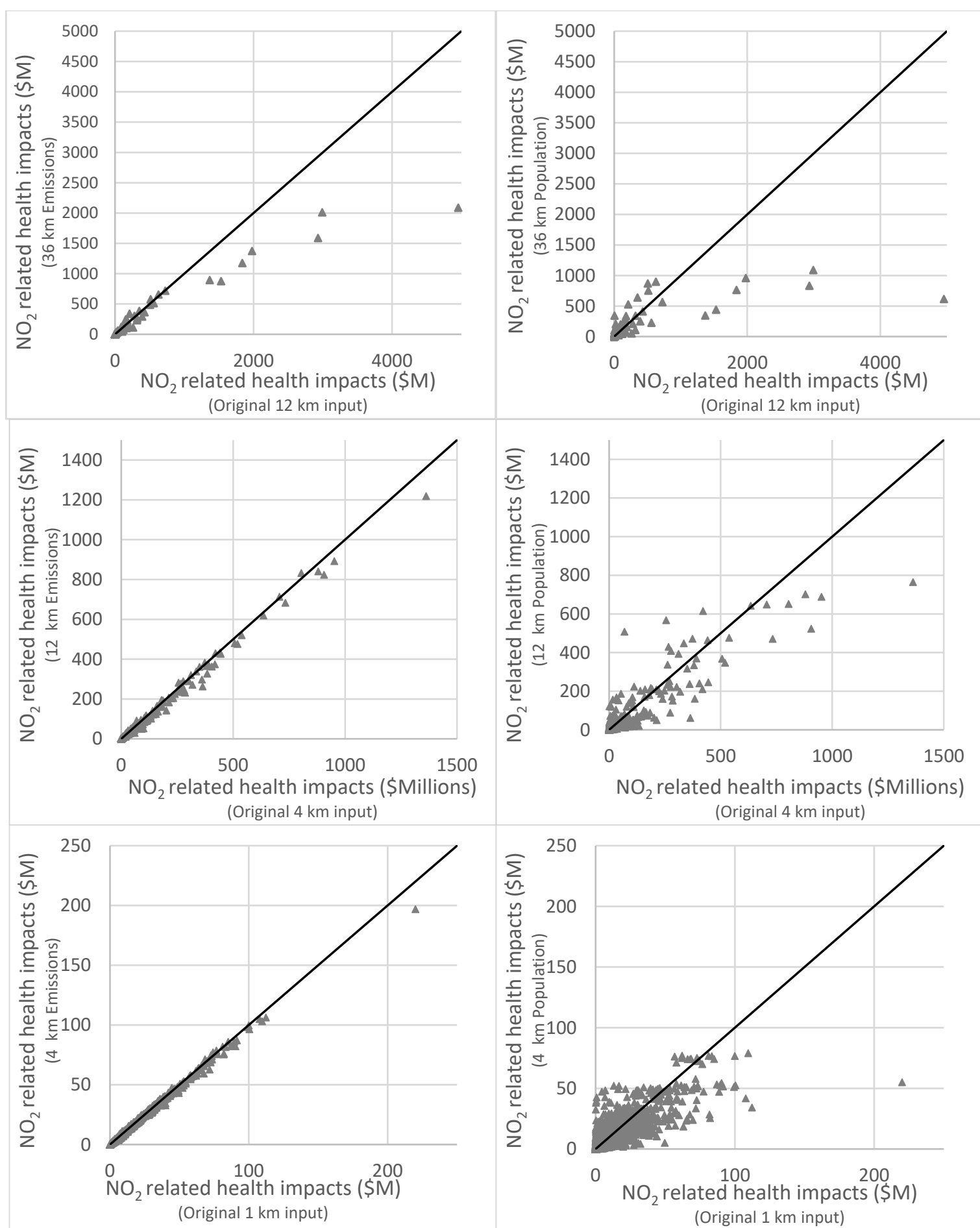

Figure 5-11 Scatter plots comparing the monetized health impacts due to chronic $\mathrm{NO}_{2}$ exposure results generated at all fine resolution with coarse emissions (left) and population (right), for resolution variation scenarios of 12 and $36 \mathrm{~km}, 4$ and $12 \mathrm{~km}$, and 1 and $4 \mathrm{~km}$ (top to bottom, respectively).

The domain-wide total health impacts due to chronic $\mathrm{NO}_{2}$ exposure are displayed in Table 5-8 for all resolution combinations and scenarios. The most drastic domain-wide impacts were seen 
between 12 and $36 \mathrm{~km}$ resolution combinations, specifically the $45 \%$ decrease in total domain health impacts at $36 \mathrm{~km}$ population compared to $12 \mathrm{~km}$ population. The difference in domainwide total health impacts between altering the resolution of inputs between $1 \mathrm{~km}$ and $4 \mathrm{~km}$ did not change drastically, with the largest impact being that of population which only decreased domain-wide health impacts by $3 \%$ from $\$ 27.1$ billion to $\$ 26.2$ billion. This may be because a decrease in emissions and/or population in one grid cells results in an increase in a subsequent cell, and as such, the domain-wide total is not heavily impacted. There are lower spatial gradients in populations and/or emissions between 1 and $4 \mathrm{~km}$ as neighborhoods and land use do not drastically change in this distance, while there can be drastic changes between 12 and $36 \mathrm{~km}$ areas. For example, the area over the lake (low emissions, zero population) does not influence the 1 and $4 \mathrm{~km}$ scenarios as drastically as it does the 12 and $36 \mathrm{~km}$ scenarios, as not as high of an area within $4 \mathrm{~km}$ occurs over the water. The original all-12 km input results are lower than those all 1 or $4 \mathrm{~km}$ input results.

Table 5-7. Domain-wide total annual health impacts due to exposure to $\mathrm{NO}_{2}$

\begin{tabular}{|c|c|c|c|c|}
\hline \multirow[b]{2}{*}{ Scenario } & \multicolumn{4}{|c|}{ Domain-wide total annual monetized health impacts $\left(\$ 10^{9}\right)$} \\
\hline & Original & $\begin{array}{c}\text { Coarse } \\
\text { Emissions } \\
\end{array}$ & $\begin{array}{c}\text { Coarse } \\
\text { Meteorology } \\
\end{array}$ & $\begin{array}{c}\text { Coarse } \\
\text { Population } \\
\end{array}$ \\
\hline $\begin{array}{l}12 \text { and } 36 \\
\mathrm{~km}\end{array}$ & 24.5 & 16.7 & 24.4 & 13.4 \\
\hline $\begin{array}{c}4 \text { and } 12 \\
\mathrm{~km}\end{array}$ & 28.7 & 26.9 & 29.4 & 25.5 \\
\hline $\begin{array}{c}1 \text { and } 4 \\
\text { km }\end{array}$ & 27.1 & 26.7 & 27.3 & 26.2 \\
\hline
\end{tabular}

Table 5-9 displays the monetized annual health impacts due to chronic $\mathrm{NO}_{2}$ exposure $\mathrm{RMSD}$ results. One mustn't assume that because the RMSD of the $1 \mathrm{~km}$ or $4 \mathrm{~km}$ resolution scenarios 
be substantially lower than those of the $12 \mathrm{~km}$ scenarios suggests that they perform vastly better, as health impacts are extensive parameters, i.e. the total health impacts in a small grid cell are lower than the total in a large grid cell. The resolution of population had the largest impact on all resolution combinations, followed by emissions, and lastly meteorology.

Emissions having a greater influence on health impacts than meteorology is consistent with the influence seen on concentration predictions.

Table 5-8. RMSD for the monetized health impacts due to $\mathrm{NO}_{2}$ exposure for each input resolution scenario, displayed in logarithmic scale.

\begin{tabular}{cccc}
\cline { 2 - 4 } Scenario & \multicolumn{3}{c}{ RMSD (\$) } \\
\cline { 2 - 4 } & $\begin{array}{c}\text { Coarse } \\
\text { Emissions }\end{array}$ & $\begin{array}{c}\text { Coarse } \\
\text { Meteorology }\end{array}$ & $\begin{array}{c}\text { Coarse } \\
\text { Meteorology } \\
\text { \& Emissions }\end{array}$ \\
\hline $\mathbf{1}$ and $\mathbf{~ k m}$ & $4.54 \times 10^{5}$ & $2.67 \times 10^{5}$ & $4.08 \times 10^{6}$ \\
$\mathbf{4}$ and $12 \mathbf{~ k m}$ & $1.17 \times 10^{6}$ & $1.10 \times 10^{6}$ & $5.17 \times 10^{7}$ \\
$\mathbf{1 2}$ and $36 \mathbf{~ k m}$ & $4.79 \times 10^{7}$ & $1.26 \times 10^{7}$ & $6.32 \times 10^{8}$
\end{tabular}

\subsubsection{Fine Particulate Matter Health Impact Results}

The change in maxima 24-hour $\mathrm{PM}_{2.5}$ health impacts when using coarse emissions consistently decreased for all resolution combinations, correlating with the decreases in concentrations. The location in which the maximum $\mathrm{PM}_{2.5}$ concentration occurs and the location in which the maximum health impacts occur are not consistent, as the maximum concentration occurs around the Toronto Pearson Airport, while the maximum health impacts occur downtown where population densities are high (i.e. high exposure). When looking at the difference plots from health impacts (Figures 5-44, 5-46, and 5-48) compared to those for concentrations (Figures 5-20, 5-22 and 5-24), the sign of the difference due to an input parameter is consistent 
between concentration and health impacts, however, the magnitudes vary drastically as health impacts are also dependent on population.

Coarse meteorology resulted in a decrease in the maximum health impacts for the 12 and 36 $\mathrm{km}$ resolution combination, however it caused an increase for the 4 and 12 , and 1 and $4 \mathrm{~km}$ resolution combinations. As seen with emissions, the location in which the maximum differences occurring due to meteorology input resolution for concentrations and for health impact estimates are not consistent. The maximum concentration impacts were not in locations with the highest population densities. A small change in the concentration in a highly populated area magnifies the impact. The sign of the difference due to an input parameter is consistent between concentration and health impacts, however, the magnitudes vary drastically as health impacts are also dependent on population.

The resolution of population had the greatest influence on $\mathrm{PM}_{2.5}$ exposure related health impacts for all resolution combinations. The highest health impacts were all underestimated (Figure 5-12 and Figure 5-13). Consequently, some of the areas with low health impacts were overpredicted. The location of the highest health impacts were those which had the highest population, averaging population over a coarse resolution reduces these maximum populations, while causing locations of low populations to be overestimated, i.e. overestimating exposure impacts. 


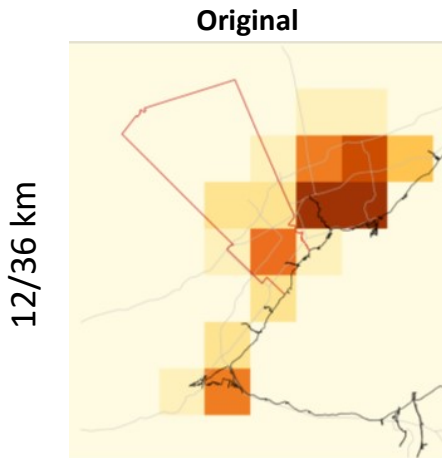

Maximum: \$14.8B

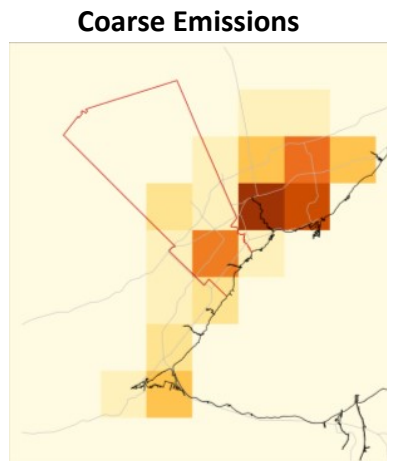

Maximum: \$10.5B

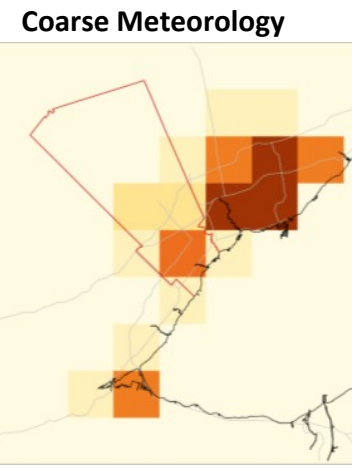

Maximum: $\$ 13.6 \mathrm{~B}$

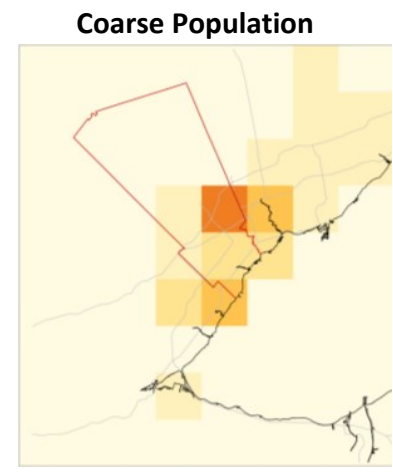

Maximum: \$6.1B

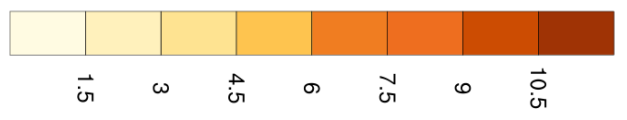

$\$ 10^{9} /$ year

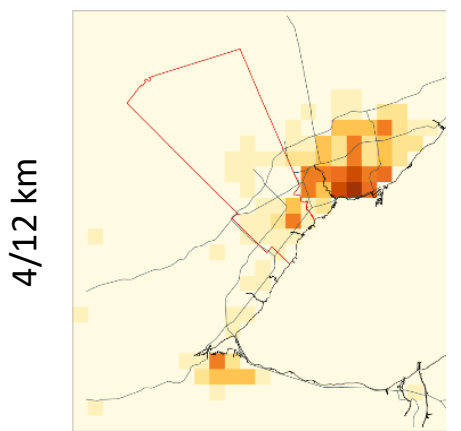

Maximum: \$3.45B

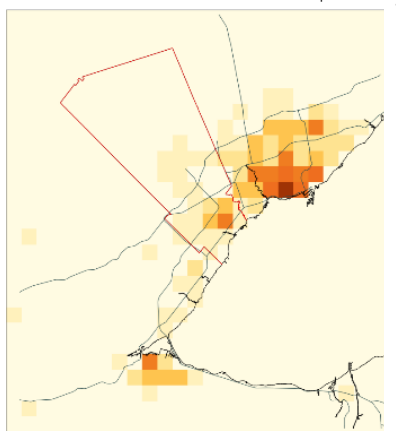

Maximum: \$3.18B

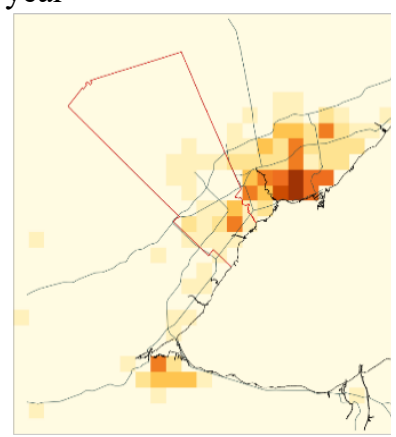

Maximum: \$3.81B

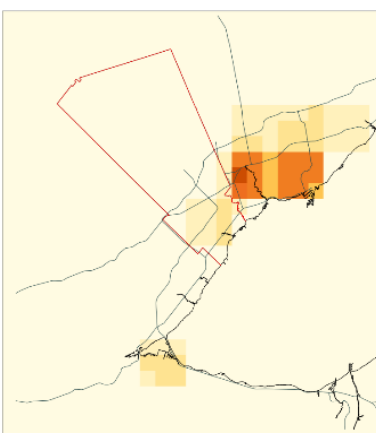

Maximum: \$0.512B
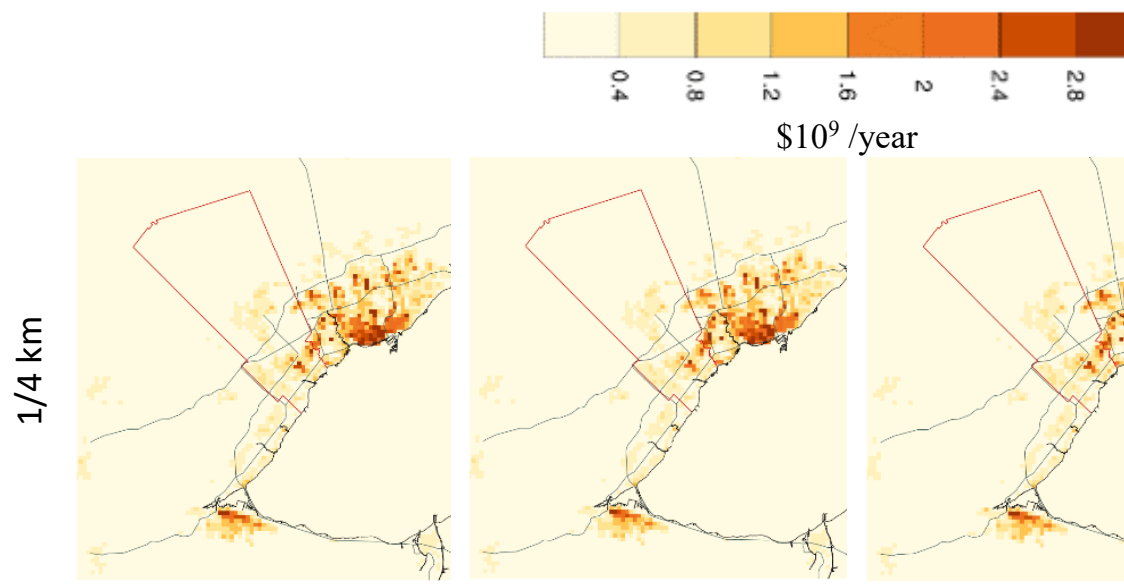

Maximum: \$596.4M

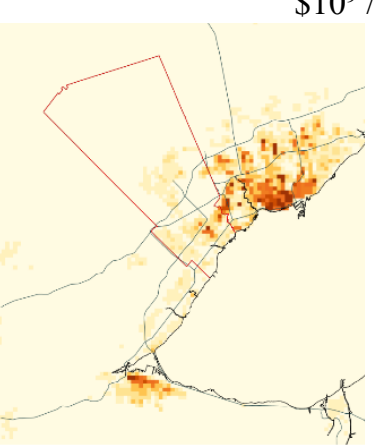

Maximum: $\$ 530.1 \mathrm{M}$

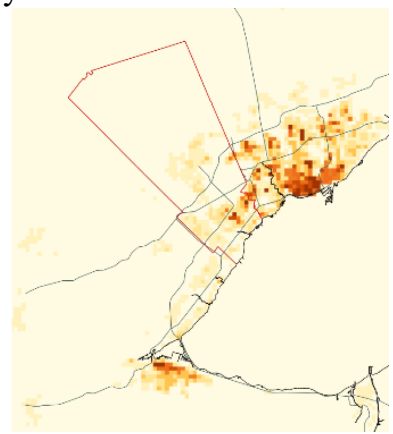

Maximum: $\$ 608.6 \mathrm{M}$

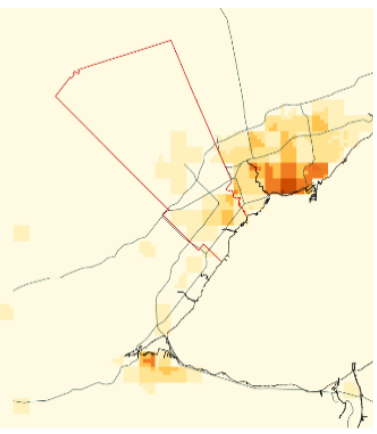

Maximum: \$219.0M

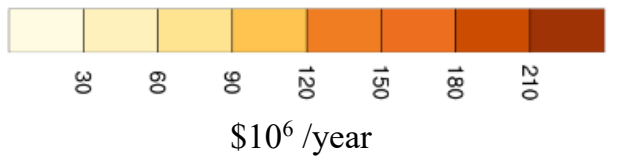

Figure 5-12. Monetized annual health impacts due to Chronic $\mathrm{PM}_{2.5}$ exposure from various fine and coarse input resolution variation scenarios of 12 and $36 \mathrm{~km}, 4$ and $12 \mathrm{~km}$, and 1 and $4 \mathrm{~km}$ (top to bottom, respectively) 


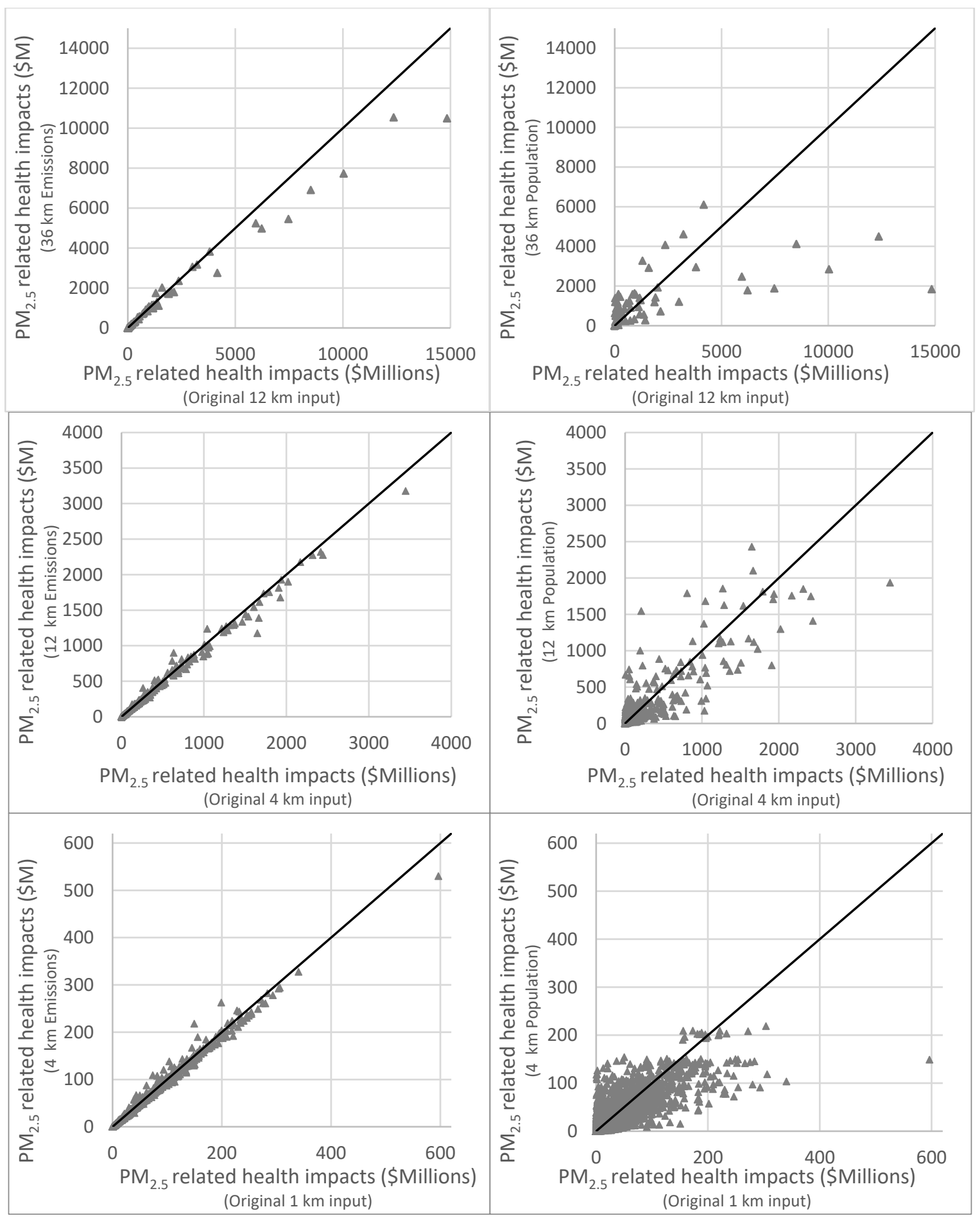

Figure 5-13 Scatter plots comparing the monetized health impacts due to chronic $\mathrm{PM}_{2.5}$ exposure results generated at all fine resolution with coarse emissions (left) and population (right), for resolution variation scenarios of 12 and $36 \mathrm{~km}, 4$ and $12 \mathrm{~km}$, and 1 and $4 \mathrm{~km}$ (top to bottom, respectively). 
The domain-wide total health impacts due to chronic $\mathrm{PM}_{2.5}$ exposure are displayed in Table 510 for all resolution combinations and scenarios. The most drastic domain-wide impacts were seen between 12 and $36 \mathrm{~km}$ resolution combinations, specifically the $33 \%$ decrease in total domain health impacts at $36 \mathrm{~km}$ population compared to $12 \mathrm{~km}$ population. Similar to the findings for $\mathrm{NO}_{2}$, the difference in domain-wide total health impacts between altering the resolution of inputs between $1 \mathrm{~km}$ and $4 \mathrm{~km}$ did not change drastically, with the largest impact being that of population which only decreased domain-wide health impacts by $3 \%$ from $\$ 107.1$ billion to $\$ 104.2$ billion. As explained for both $\mathrm{O}_{3}$ and $\mathrm{NO}_{2}$, this may be attributed to the fact that spatial gradients are not as drastic between 1 and $4 \mathrm{~km}$ as they are between 12 and $36 \mathrm{~km}$. The original all fine cases did not vary drastically whether modelled at $1 \mathrm{~km}, 4 \mathrm{~km}$, or at $12 \mathrm{~km}$. This is consistent with results found from Arunachalam et al. (2011), who found that there was not a drastic difference between domain aggregated total health risks related to $\mathrm{PM}_{2.5}$ exposure at resolutions of 36,12 and $4 \mathrm{~km}$. In contrast, there has been other research which found that coarser resolutions underestimate health impacts due to $\mathrm{PM}_{2.5}$ exposure (Thompson et al., 2014; Li et al., 2015).

Table 5-9 Domain-wide total annual health impacts due to exposure to $\mathrm{PM}_{2.5}$

\begin{tabular}{ccccc}
\cline { 2 - 5 } & \multicolumn{3}{c}{ Domain-wide total annual monetized health impacts $\mathbf{( \$ 1 0} \mathbf{9})$} \\
\cline { 2 - 5 } Scenario & Original & $\begin{array}{c}\text { Coarse } \\
\text { Emissions }\end{array}$ & $\begin{array}{c}\text { Coarse } \\
\text { Meteorology }\end{array}$ & $\begin{array}{c}\text { Coarse } \\
\text { Population }\end{array}$ \\
\hline $\begin{array}{c}\mathbf{1 2} \text { and } \mathbf{3 6} \\
\mathbf{~ k m}\end{array}$ & 107.1 & 91.1 & 103.2 & 71.2 \\
$\mathbf{4}$ and $\mathbf{1 2} \mathbf{~ k m}$ & 111.0 & 107.8 & 111.9 & 104.3 \\
$\mathbf{1}$ and $\mathbf{4} \mathbf{~ k m}$ & 107.1 & 106.0 & 107.4 & 104.2
\end{tabular}


Table 5-11 displays the monetized annual health impacts due to chronic $\mathrm{PM}_{2.5}$ exposure RMSD results. The drastic difference in magnitude between resolution combinations can be attributed to the fact that health impacts are an extensive parameter. The resolution of population had the largest impact on all resolution combinations, followed by emissions, and lastly meteorology. Emissions having a greater influence on health impacts than meteorology is consistent with the influence seen on concentration predictions.

Table 5-10. RMSD for the monetized health impacts due to $\mathrm{PM}_{2.5}$ exposure for each input resolution scenario, displayed in logarithmic scale

\begin{tabular}{cccc}
\cline { 2 - 4 } Scenario & \multicolumn{3}{c}{ RMSD (\$) } \\
\cline { 2 - 4 } & $\begin{array}{c}\text { Coarse } \\
\text { Emissions }\end{array}$ & $\begin{array}{c}\text { Coarse } \\
\text { Meteorology }\end{array}$ & $\begin{array}{c}\text { Coarse } \\
\text { Population }\end{array}$ \\
\hline $\mathbf{1}$ and $4 \mathbf{~ k m}$ & $1.90 \times 10^{6}$ & $9.68 \times 10^{5}$ & $1.45 \times 10^{7}$ \\
$\mathbf{4}$ and $12 \mathbf{~ k m}$ & $3.62 \times 10^{7}$ & $3.70 \times 10^{7}$ & $1.88 \times 10^{8}$ \\
$\mathbf{1 2}$ and $36 \mathbf{~ k m}$ & $6.93 \times 10^{8}$ & $3.58 \times 10^{8}$ & $2.22 \times 10^{9}$
\end{tabular}

\subsubsection{Resolution Health Impact Comparisons}

Regardless of the species $\left(\mathrm{O}_{3}, \mathrm{NO}_{2}\right.$, and $\left.\mathrm{PM}_{2.5}\right)$ and regardless of the resolution combinations (1 and $4 \mathrm{~km}, 4$ and $12 \mathrm{~km}$, or 12 and $36 \mathrm{~km}$ ), population consistently had the greatest impact on health estimates. Health impacts are directly proportional to populations, causing them to be highly sensitive to their input. Population gradients are also strong, as neighborhoods can alter drastically in population densities over short distances. Furthermore, the location under analysis has the highest populations near the waterfront, i.e. the locations with the highest populations are adjacent to locations with zero population, causing significant dilution when averaging occurs between the two land uses. 
Following population, the health impacts were most impacted by the input that the concentration impacts were most impacted for. Nitrogen dioxide and $\mathrm{PM}_{2.5}$ were more heavily impacted by the resolution of emissions followed by meteorology, whereas $\mathrm{O}_{3}$ was more heavily impacted by the resolution of meteorology followed by emissions. The ratio in which health impacts were influenced by resolution was not proportional to the influences on concentration as the exposure relationship is exponential to concentration and is also a function of population.

Nitrogen dioxide and $\mathrm{PM}_{2.5}$ saw decreases in domain-wide health impacts due to coarse emissions, consistent with research conducted by Punger and West (2013) who found that coarse resolutions caused a decrease in overall health impacts (however, this research looked at all inputs at coarse resolution). The all $12 \mathrm{~km}$ input resolution scenario for $\mathrm{O}_{3}$ had a higher domain-wide health impact than the finer resolutions, a trend consistent with Punger and West (2013).

\subsubsection{Discussion of Uncertainties}

There are various uncertainties and limitations inherently associated with atmospheric modelling, emission characterization, epidemiology data, and mortality valuation. Uncertainties in atmospheric models exist from the representation of complex atmospheric interactions. Russell and Dennis (2000) found that CTM results were highly impacted by uncertainties within the inputted emissions and meteorology. Epidemiology data also contains uncertainties, for 
example, the concentration response function assumes a uniform response to air quality across the population.

A limitation of this research is the short modelling period of two days within July. These results are extrapolated to annual values. Two days within July may not adequately capture differing pollution patterns seen within the month, nor do they accurately represent yearly pollution patterns. Because of this, results presented for $\mathrm{O}_{3}$ may be overpredicted as $\mathrm{O}_{3}$ concentrations during summer months are higher than those of winter months as $\mathrm{O}_{3}$ is photochemically produced. The variety of meteorology in the span of a year cannot be captured within a twoday period. As such, the results may not be applicable to differing time periods. It is suggested that future research simulate longer episodes and contains periods outside of just the summer.

The small domain and specific land-uses (e.g. city on a large body of water) may not have results that are representative of all locations. Future research could look at a larger domain, or more than one domain with differing land uses. The results may be applicable for other domains in which an urban core exists on the coast of a large body of water, but it is expected that in-land domains would have altering results.

\subsection{Conclusions}

Nitrogen dioxide and $\mathrm{PM}_{2.5}$ 24-hour average concentrations were more influenced by the input resolution of emissions than they were meteorology. Both species are relatively short-lived and are primary (or partially, in the case of $\mathrm{PM}_{2.5}$ ) pollutants, causing them to have high concentrations nearby emission sources. The short dispersal ranges (a function of their 
relatively short lifetimes) of these pollutants cause them to have high spatial concentration gradients near emission sources. These factors contribute to their high sensitivity to the resolution of emissions. In specific locations (surrounding the airport), $\mathrm{PM}_{2.5}$ saw higher impacts to altering the resolution of emissions between 1 and $4 \mathrm{~km}$ than it did between 4 and $12 \mathrm{~km}$ or 12 and $36 \mathrm{~km}$. This suggests that even at $4 \mathrm{~km}$ resolution, the high spatial gradients in emissions were inadequately captured. When modeling $\mathrm{PM}_{2.5}$, one should utilize high resolution emissions despite the high computational time and storage as using coarse emissions puts the accuracy of $\mathrm{PM}_{2.5}$ results at risk. When the few locations (only two $1 \times 1 \mathrm{~km}$ cells) of significantly high $\mathrm{PM}_{2.5}$ emissions were removed, the influence to resolution of emissions was more consistent, or slightly lower, with that of $\mathrm{NO}_{2}$. The findings within this research suggest that a 4 $\mathrm{km}$ resolution may be sufficient to predict $\mathrm{NO}_{2}$.

Ozone DM8A concentrations were more influenced by the input resolution of meteorology than of emissions. Ozone is a secondary species with a lifetime longer than those of $\mathrm{NO}_{2}$ or $\mathrm{PM}_{2.5}$, and as such, its concentration is more spatially homogenous, contributing to its lower sensitivity to emissions. Ozone is photochemically produced and has an increased formation rate at high temperatures, causing it to be directly impacted by meteorology (cloud cover, albedo, and temperature). The resolution of the CTM saw a large impact between the $1 \mathrm{~km}$ and $4 \mathrm{~km}$ models. The $1 \mathrm{~km}$ results were overall lower across the domain than those generated at higher resolutions. The complex chemistry involved in $\mathrm{O}_{3}$ production may not be accurately represented at resolutions as small as $1 \mathrm{~km}$. The $36 \mathrm{~km}$ input resolutions saw the most drastic 
changes in concentrations, and as such it is suggested that a resolution of 12 or $4 \mathrm{~km}$ be used when modelling $\mathrm{O}_{3}$.

Health impact results, characterized by monetized mortality due to chronic exposure to the pollutants of study, were most heavily influenced by the resolution of the population input, regardless of the species of analysis or the resolution scenario. Second to population, the resolution of the input that had the highest influence on concentration had the highest influence on health impacts. For $\mathrm{NO}_{2}$ and $\mathrm{PM}_{2.5}$, emissions influenced health impacts more than meteorology, while for $\mathrm{O}_{3}$, meteorology influenced health impacts more than emissions. Health impacts related to $\mathrm{O}_{3}$ had an overall lower impact due to the resolution of meteorology or emissions than $\mathrm{PM}_{2.5}$ and $\mathrm{NO}_{2}$ did. Fine particulate matter and $\mathrm{NO}_{2}$ are species that have localized concentrations near emission sources. The location of emissions sources often corresponds to urban areas with high populations, hence high exposure. This causes a direct link between the impact of resolution on concentrations and on health impacts. This does not hold true for $\mathrm{O}_{3}$, as $\mathrm{O}_{3}$ is a secondary pollutant which forms downwind from precursor emission sources, which may occur over locations of low populations. We suggest that the CTM be run at the above suggested resolutions related to the species of interest, and the population used be as fine resolution as readily available. Since health exposure equations are applied after the CTM, using a fine resolution population will not alter the computation costs of the CTM. 


\section{Chapter 5 Supplementary Material}

The $\mathrm{O}_{3}$ concentration (daily maximum 8-hour average, DM8A) results from the $12 \mathrm{~km}$ scenarios with $36 \mathrm{~km}$ input variations, along with each of their respective differences with the original all $12 \mathrm{~km}$ model run, are displayed in Figure 5-14. The scatter comparison plots for $\mathrm{DM}^{\mathrm{AA}} \mathrm{O}_{3}$ concentrations from the $12 \mathrm{~km}$ scenarios with $36 \mathrm{~km}$ input variations are displayed in Figure 5-

15.

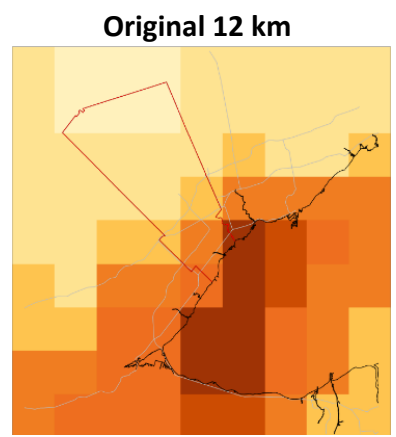

Maximum: $64.5 \mathrm{ppb}$

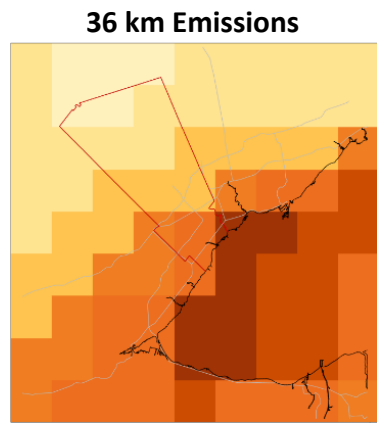

Maximum: $62.0 \mathrm{ppb}$

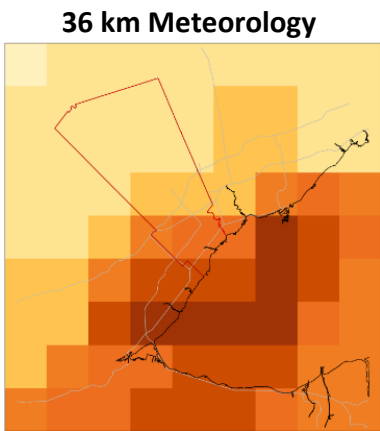

Maximum: $66.5 \mathrm{ppb}$

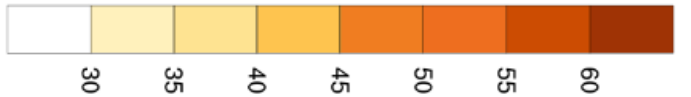

Ppb

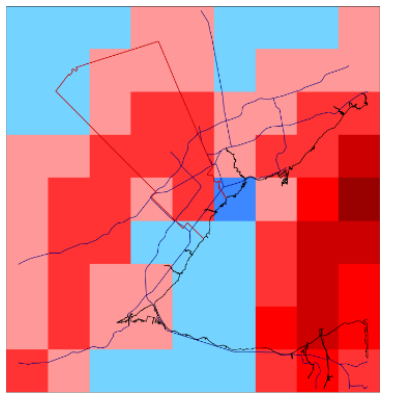

Maximum Difference: $10.1 \mathrm{ppb}$ Minimum Difference: -4.5 ppb

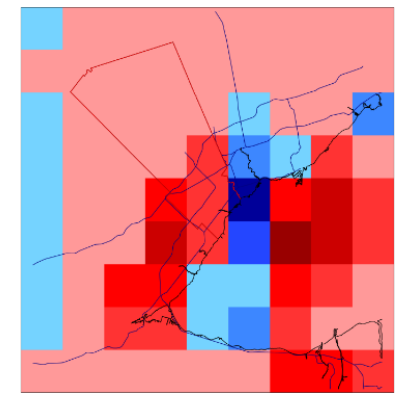

Maximum Difference: 11.0 ppb Minimum Difference: -11.6 ppb

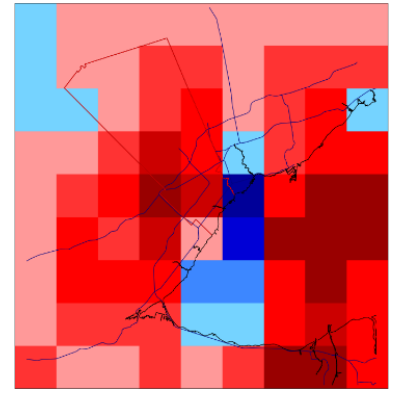

Maximum Difference: $19.2 \mathrm{ppb}$ Minimum Difference: -12.9 ppb

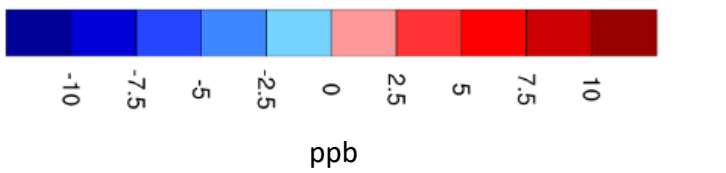

36 km Meteorology \& Emissions

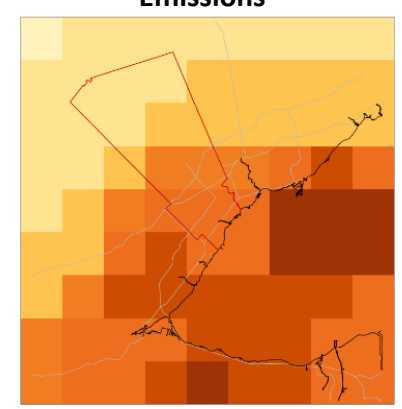

Maximum: $66.5 \mathrm{ppb}$

Difference with Original:

$\mathrm{ppb}$

Figure 5-14. Daily Maximum 8-hour average $\mathrm{O}_{3}$ concentration results from various $12 \mathrm{~km}$ (fine) and $36 \mathrm{~km}$ (coarse) input resolution variation scenarios and the differences between the respective scenario and the all fine $12 \mathrm{~km}$ model run 

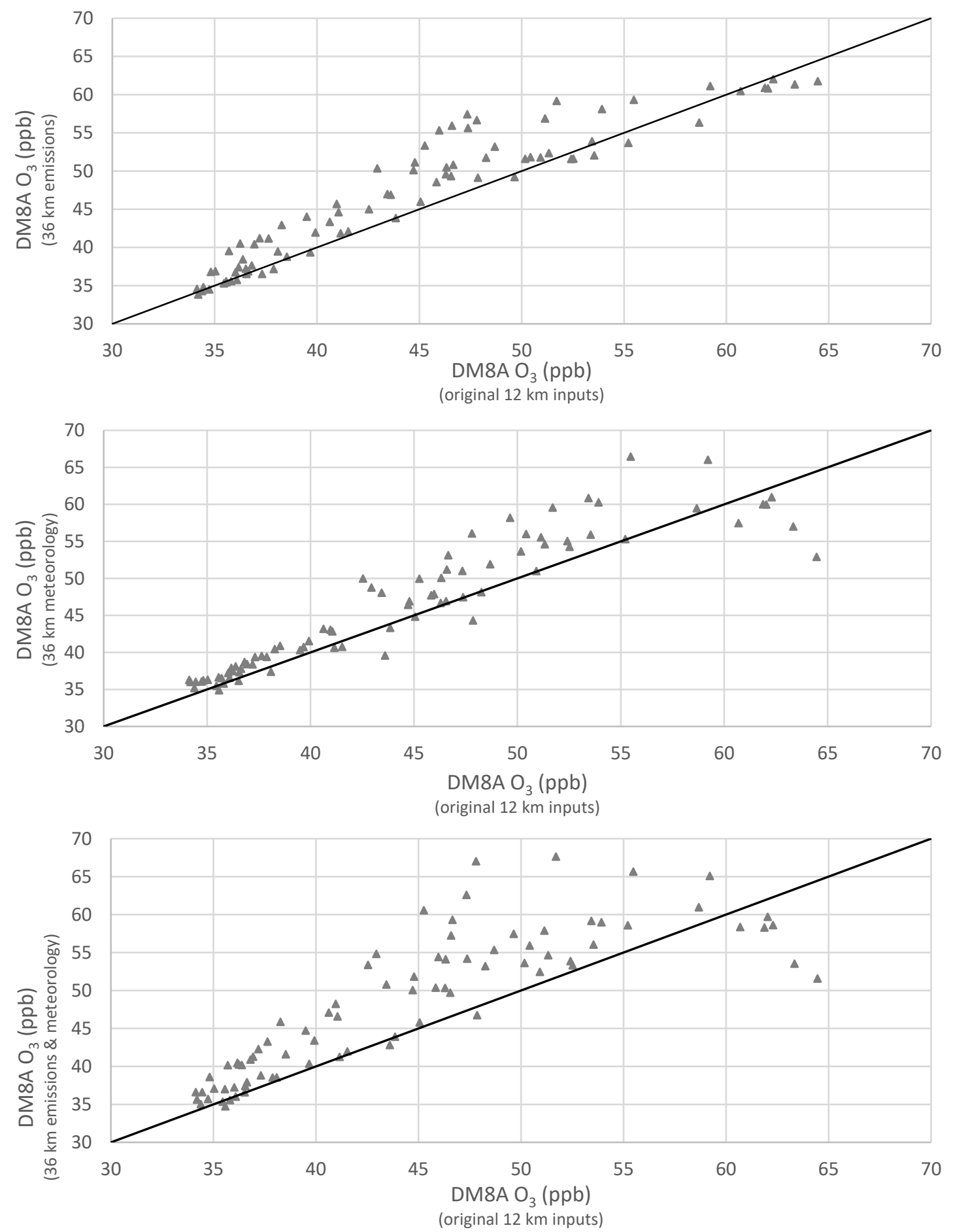

Figure 5-15. Scatter plots comparing the $\mathrm{DM} 8 \mathrm{~A} \mathrm{O}_{3}$ concentration results for combinations of fine $12 \mathrm{~km}$ resolution with coarse $36 \mathrm{~km}$ inputs: emissions (top), meteorology (middle) and both emissions and meteorology (bottom). 
The $\mathrm{O}_{3}$ concentration (daily maximum 8-hour average, $\mathrm{DM} 8 \mathrm{~A}$ ) results from the $4 \mathrm{~km}$ scenarios with $12 \mathrm{~km}$ input variations, along with each of their respective differences with the original all $4 \mathrm{~km}$ model run, are displayed in Figure 5-16. The scatter comparison plots for $\mathrm{DM}_{8 \mathrm{~A} \mathrm{O}} \mathrm{O}_{3}$ concentrations from the $4 \mathrm{~km}$ scenarios with $12 \mathrm{~km}$ input variations are displayed in Figure 5-

17.

Original 4 km

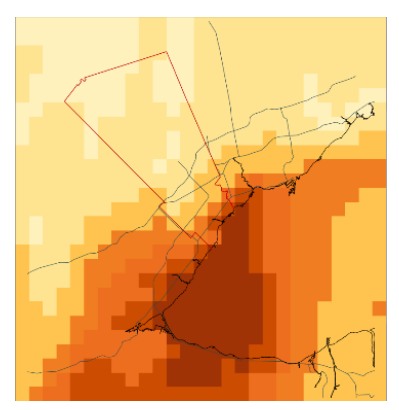

Maximum: $60.0 \mathrm{ppb}$
12 km Emissions

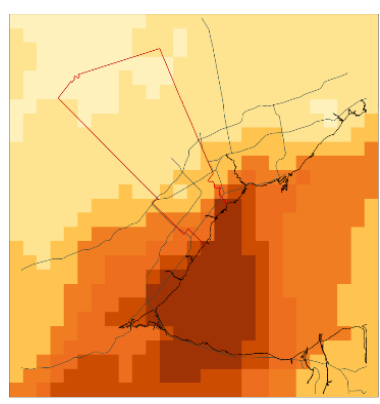

Maximum: $61.1 \mathrm{ppb}$
12 km Meteorology

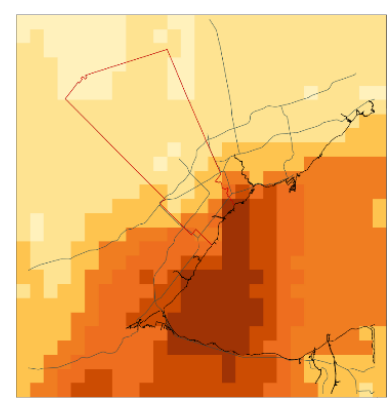

Maximum: 60.9 ppb
12 km Meteorology \& Emissions

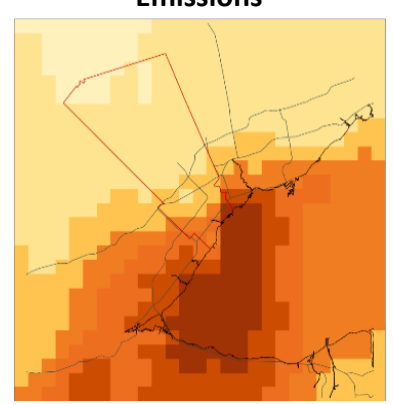

Maximum: 64.9

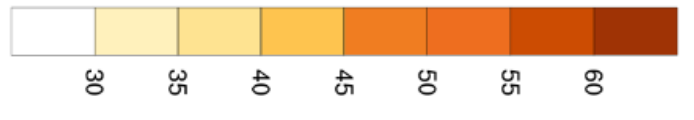

ppb

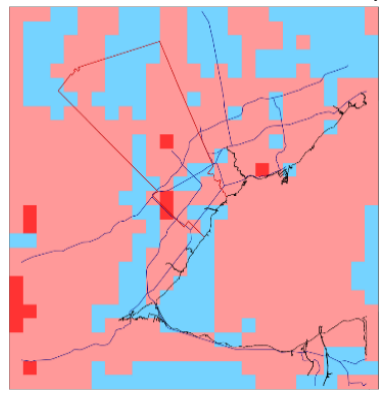

Maximum Difference: $3.0 \mathrm{ppb}$ Minimum Difference: $-1.5 \mathrm{ppb}$

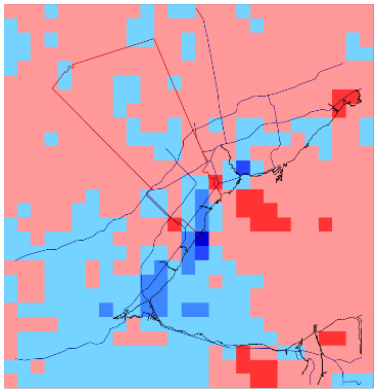

Maximum Difference: $3.9 \mathrm{ppb}$ Minimum Difference: $-7.6 \mathrm{ppb}$

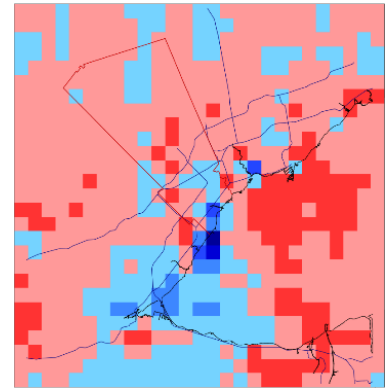

Maximum Difference: $5.0 \mathrm{ppb}$ Minimum Difference: $-8.4 \mathrm{ppb}$

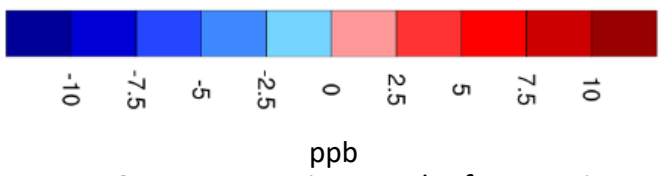

$\mathrm{ppb}$

Figure 5-16 Daily Maximum 8-hour average $\mathrm{O}_{3}$ concentration results from various $4 \mathrm{~km}$ (fine) and $12 \mathrm{~km}$ (coarse) input resolution variation scenarios and the differences between the respective scenario and the all fine $4 \mathrm{~km}$ model run 

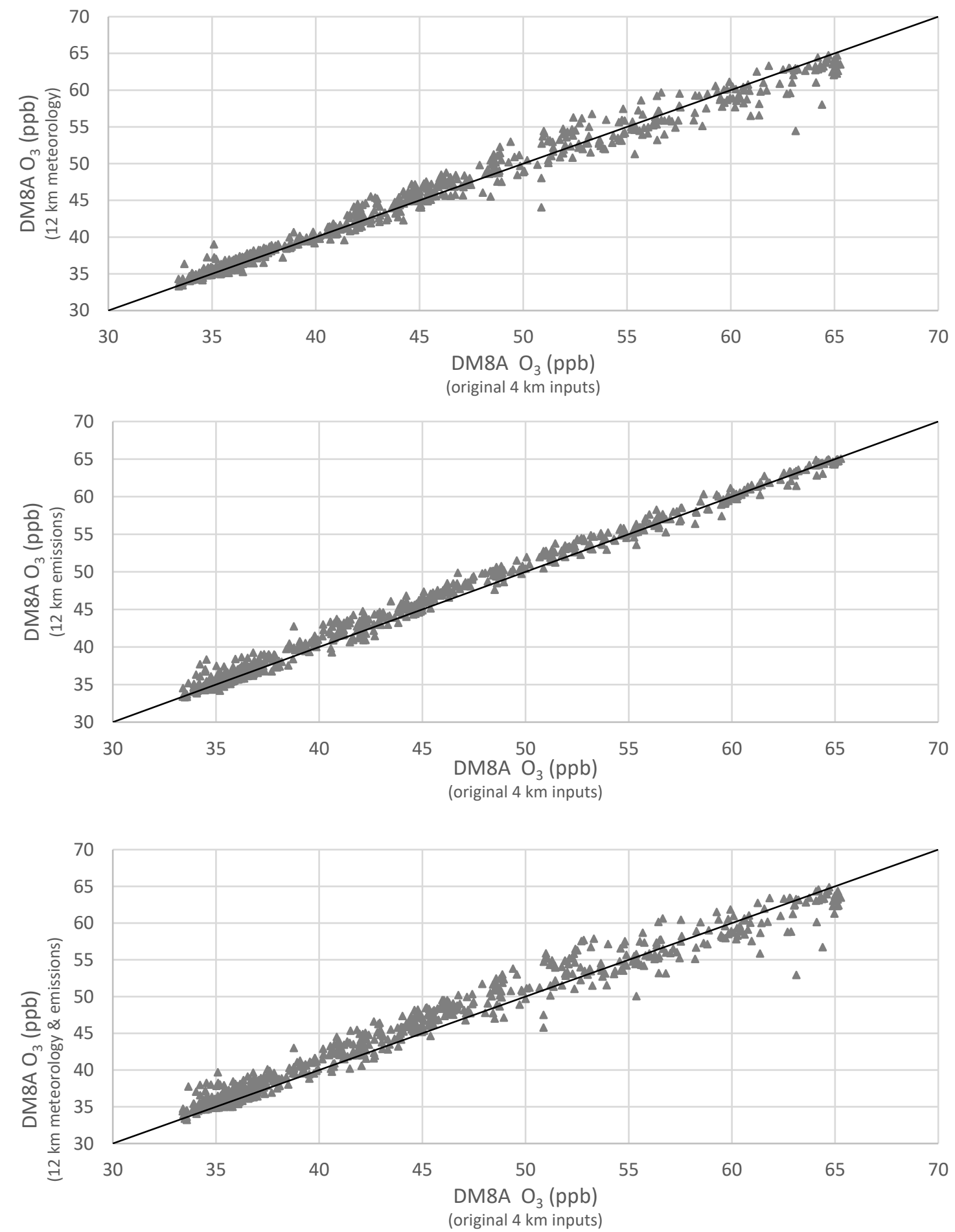

Figure 5-17. Scatter plots comparing the $\mathrm{DM} 8 \mathrm{~A} \mathrm{O}_{3}$ concentration results for combinations of fine $4 \mathrm{~km}$ resolution with coarse $12 \mathrm{~km}$ inputs: emissions (top), meteorology (middle) and both emissions and meteorology (bottom). 
The $\mathrm{O}_{3}$ concentration (daily maximum 8-hour average, DM8A) results from the $1 \mathrm{~km}$ scenarios with $4 \mathrm{~km}$ input variations, along with each of their respective differences with the original all 1 $\mathrm{km}$ model run, are displayed in Figure 5-18. The scatter comparison plots for $\mathrm{DM}_{8 \mathrm{~A}} \mathrm{O}_{3}$ concentrations from the $1 \mathrm{~km}$ scenarios with $4 \mathrm{~km}$ input variations are displayed in Figure 5-19.

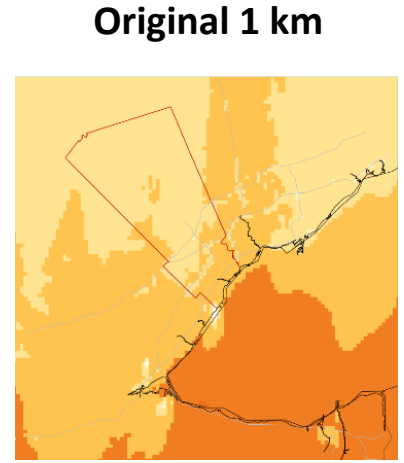

Maximum: $50.3 \mathrm{ppb}$
4 km Emissions

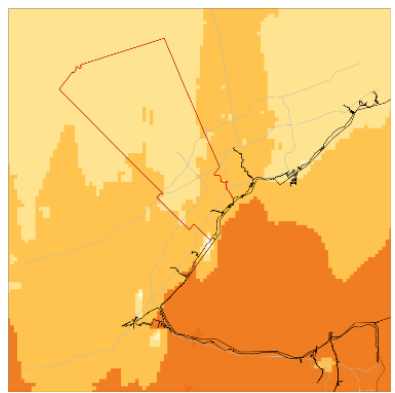

Maximum: $50.2 \mathrm{ppb}$

\section{4 km Meteorology}

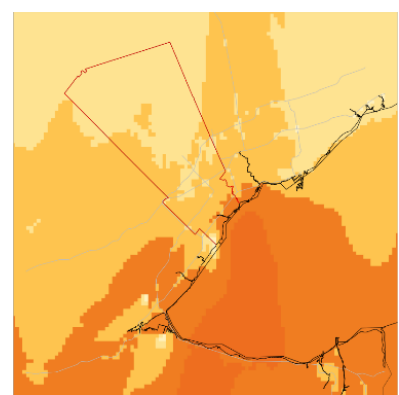

Maximum: $52.2 \mathrm{ppb}$

\section{4 km Meteorology \&} Emissions

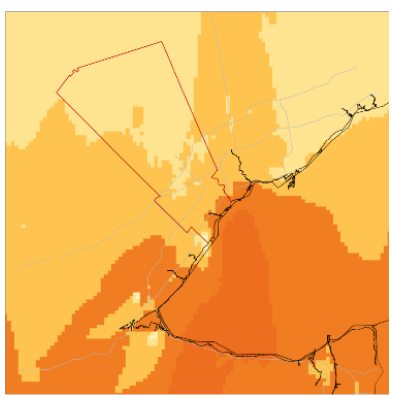

Maximum: $52.1 \mathrm{ppb}$

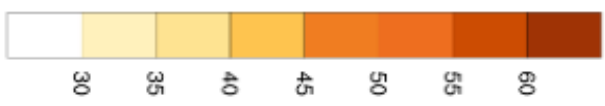

ppb

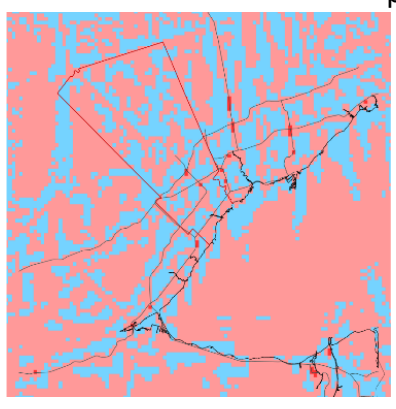

Maximum Difference: 4.94 ppb Minimum Difference: -2.10 ppb

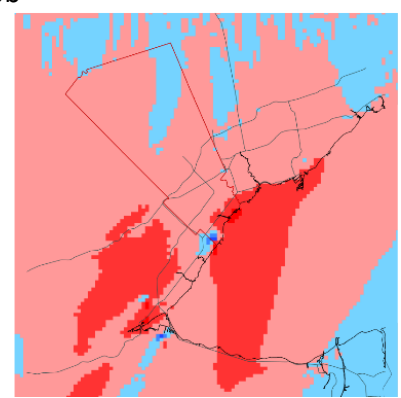

Maximum Difference: 6.59 ppb Minimum Difference: -6.61 ppb

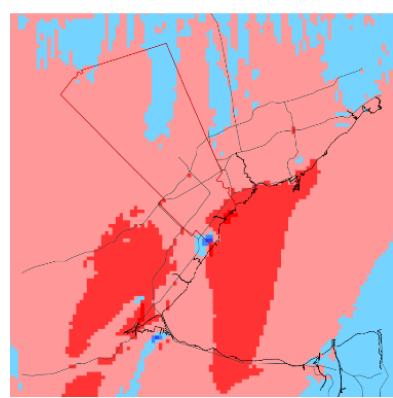

Maximum Difference: 6.80 ppb Minimum Difference: -6.80 ppb

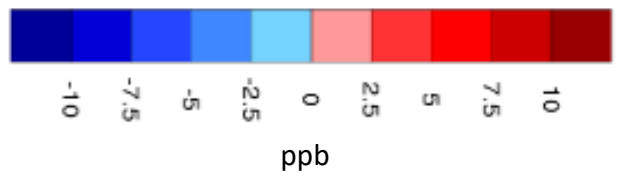

Figure 5-18. Daily Maximum 8-hour average $\mathrm{O}_{3}$ concentration results from various $1 \mathrm{~km}$ (fine) and $4 \mathrm{~km}$ (coarse) input resolution variation scenarios and the differences between the respective scenario and the all fine $1 \mathrm{~km}$ model run. 

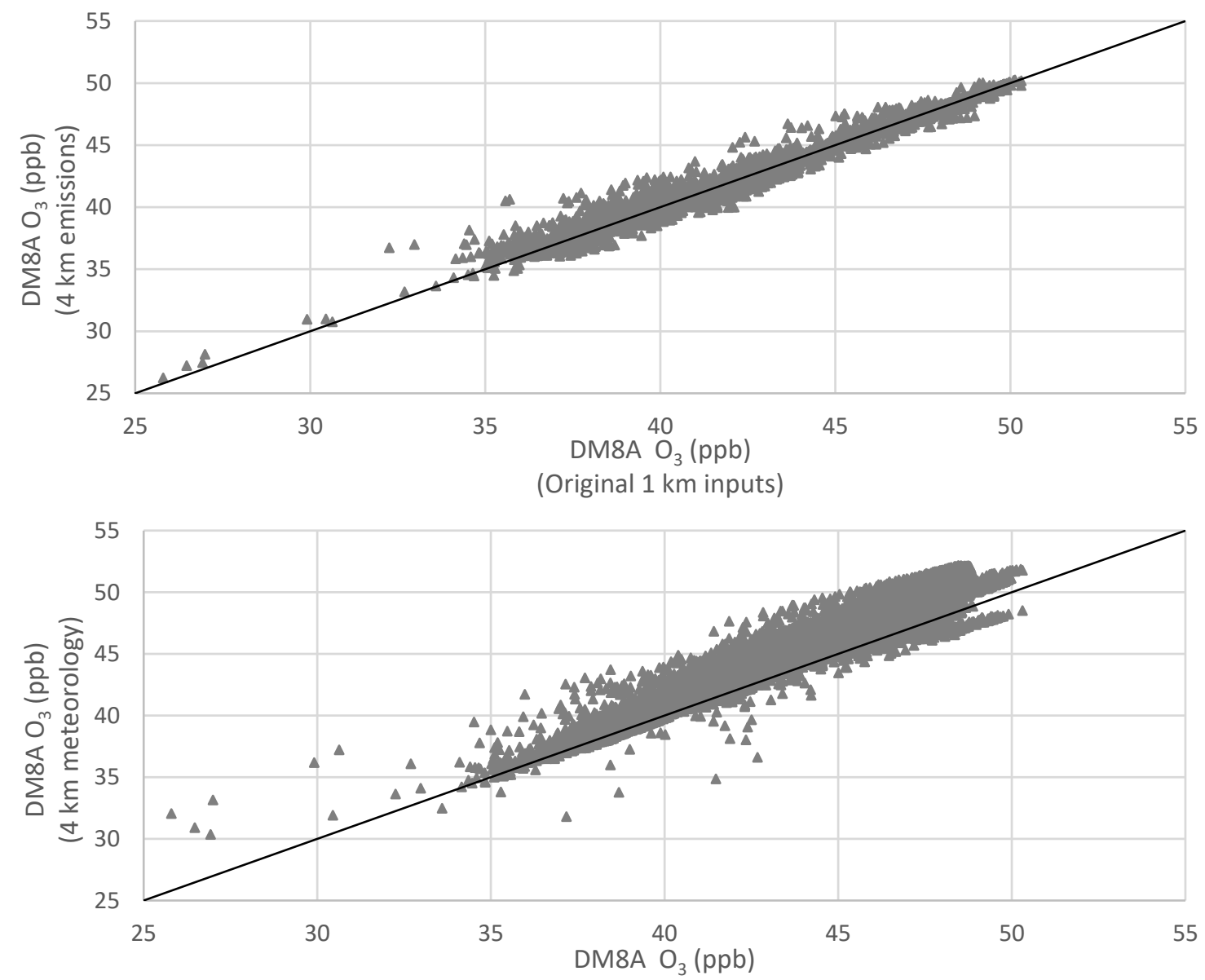

(Original $1 \mathrm{~km}$ inputs

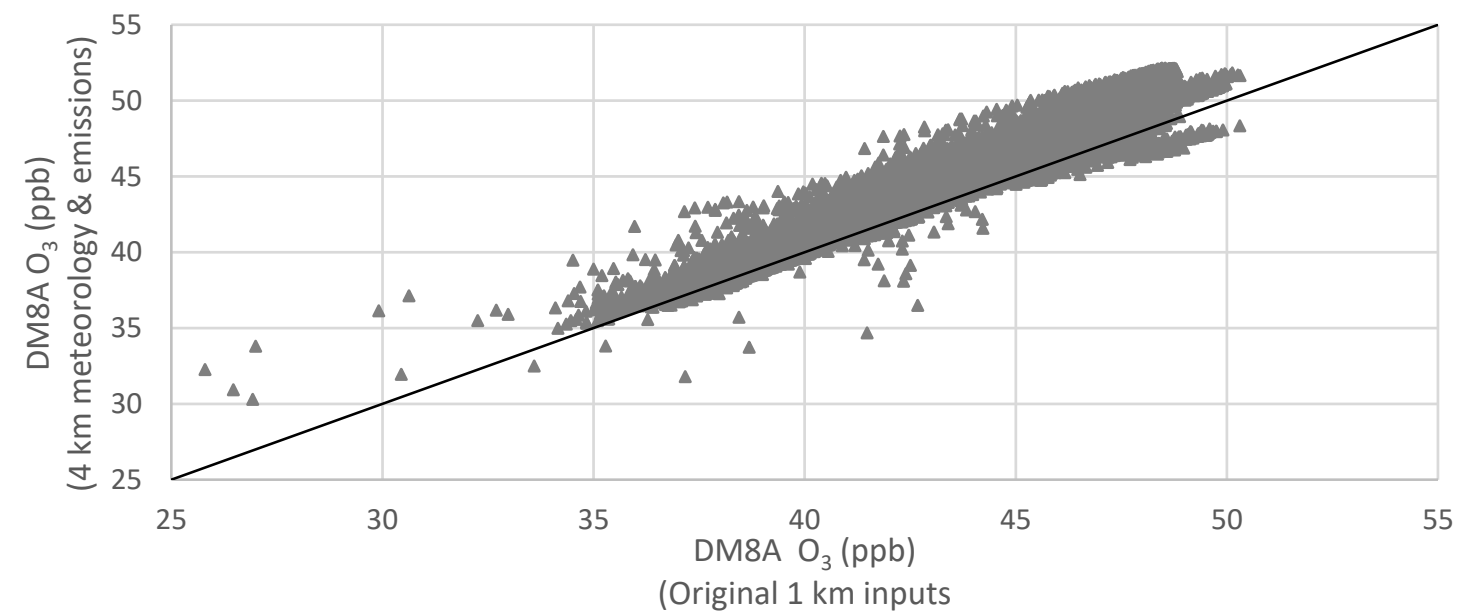

Figure 5-19. Scatter plots comparing the $\mathrm{DM} 8 \mathrm{~A} \mathrm{O}_{3}$ concentration results for combinations of fine $1 \mathrm{~km}$ resolution with coarse $4 \mathrm{~km}$ inputs: emissions (top), meteorology (middle) and both emissions and meteorology (bottom). 
The $\mathrm{NO}_{2}$ concentration (daily 24-hour average) results from the $12 \mathrm{~km}$ scenarios with $36 \mathrm{~km}$ input variations, along with their respective differences with the original all $12 \mathrm{~km}$ model run, are displayed in Figure 5-20. The scatter comparison plots for the daily 24-hour average $\mathrm{NO}_{2}$ concentrations from the $12 \mathrm{~km}$ scenarios with $36 \mathrm{~km}$ input variations are displayed in Figure 5-

21.

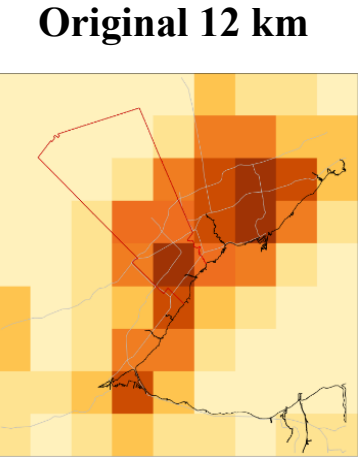

Maximum: $21.3 \mathrm{ppb}$
36 km Emissions

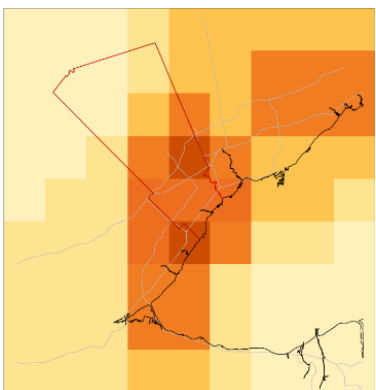

Maximum: $17.0 \mathrm{ppb}$
36 km Meteorology

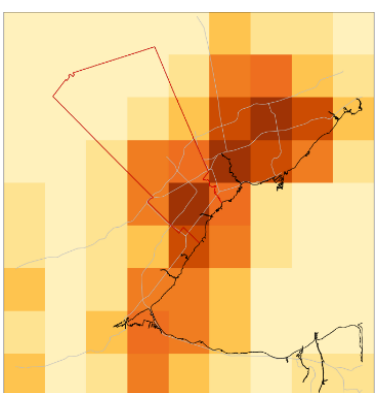

Maximum: $21.2 \mathrm{ppb}$
36 km Meteorology
\& Emissions

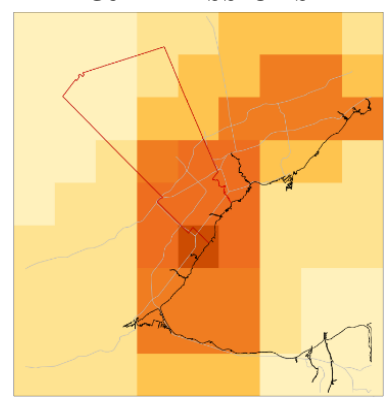

Maximum: $16.2 \mathrm{ppb}$

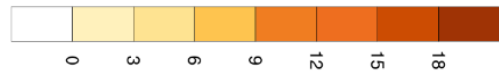

$\mathrm{ppb}$

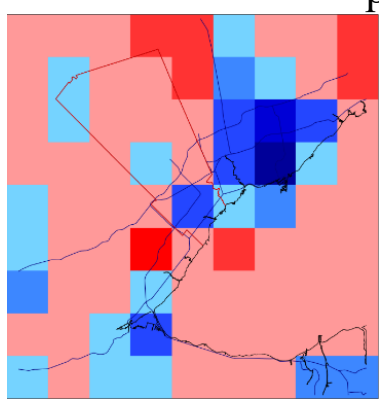

Maximum Difference: $5.4 \mathrm{ppb}$ Min. Difference: $-11.1 \mathrm{ppb}$

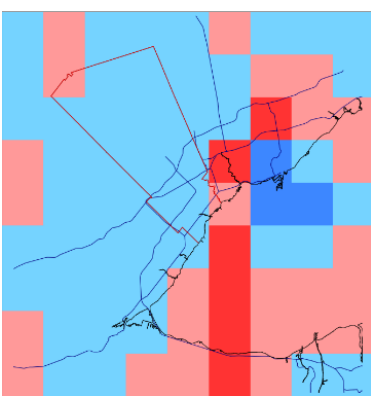

Maximum Difference: $4.2 \mathrm{ppb}$ Minimum Difference: $-4.4 \mathrm{ppb}$

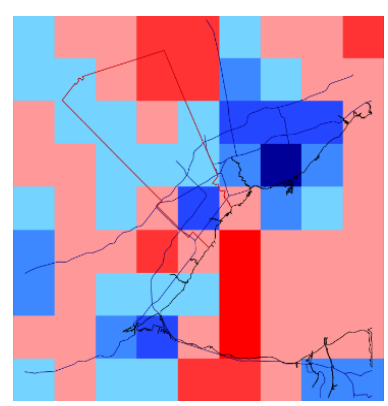

Maximum Difference: $6.8 \mathrm{ppb}$ Min. Difference: $-11.7 \mathrm{ppb}$

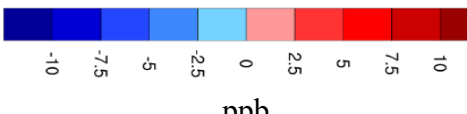

$\mathrm{ppb}$

Figure 5-20. 24-hour average $\mathrm{NO}_{2}$ concentration results from various $12 \mathrm{~km}$ (fine) and $36 \mathrm{~km}$ (coarse) input resolution variation scenarios and the differences between the respective scenario and the all fine $12 \mathrm{~km}$ model run. 

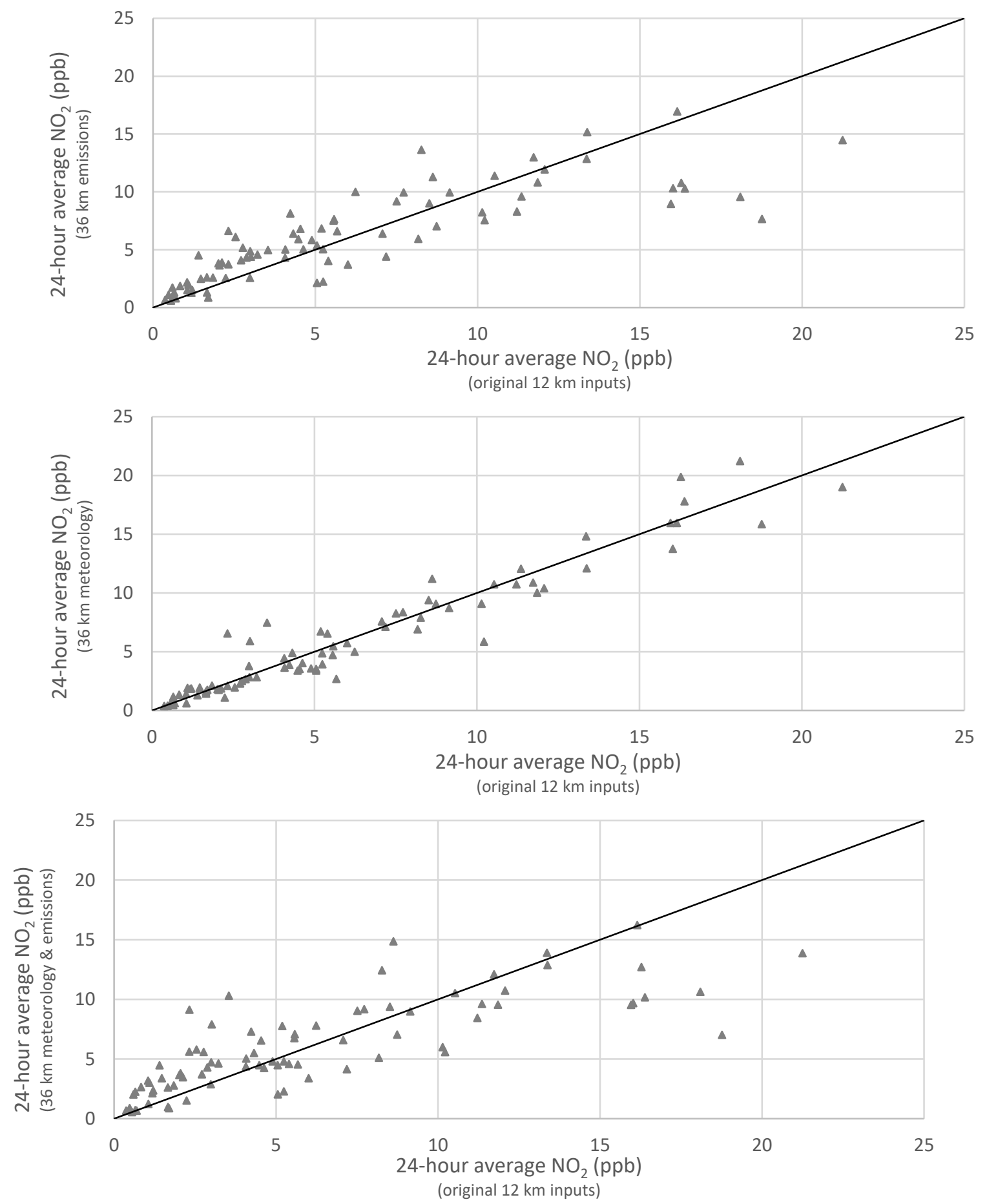

Figure 5-21. Scatter plots comparing the 24-hour average $\mathrm{NO}_{2}$ concentration results for combinations of fine $12 \mathrm{~km}$ resolution with coarse $36 \mathrm{~km}$ inputs: emissions (top), meteorology (middle), and emissions and meteorology (bottom). 
The $\mathrm{NO}_{2}$ concentration (daily 24-hour average) results from the $4 \mathrm{~km}$ scenarios with $12 \mathrm{~km}$ input variations, along with their respective differences with the original all $4 \mathrm{~km}$ model run, are displayed in Figure 5-22. The scatter comparison plots for the daily 24-hour average $\mathrm{NO}_{2}$ concentrations from the $4 \mathrm{~km}$ scenarios with $12 \mathrm{~km}$ input variations are displayed in Figure 5-

23.

\section{Original 4 km}

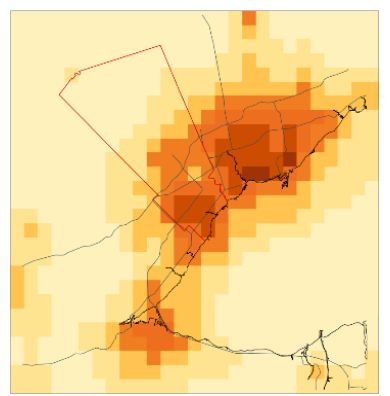

Maximum: 26.7 ppb
12 km Emissions

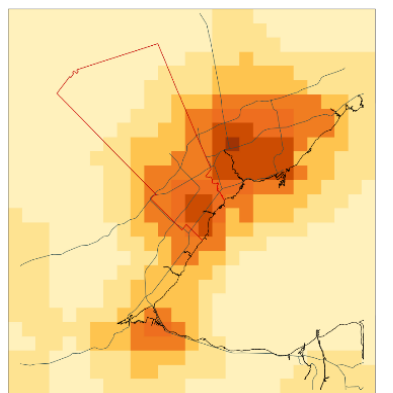

Maximum: $24.2 \mathrm{ppb}$
12 km Meteorology

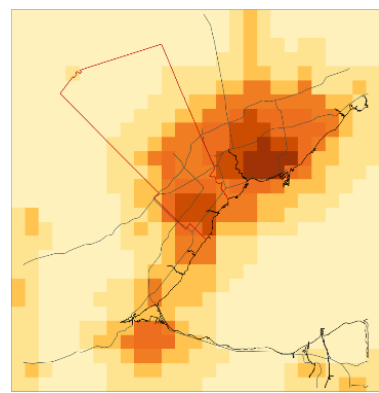

Maximum: 29.8 ppb
12 km Meteorology

\& Emissions

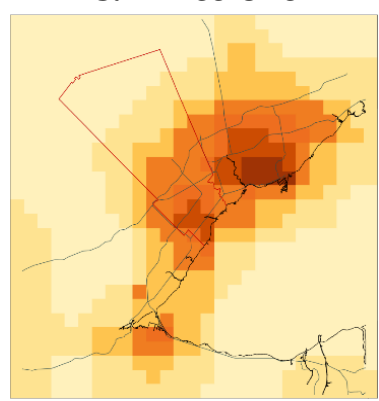

Maximum: $26.2 \mathrm{ppb}$

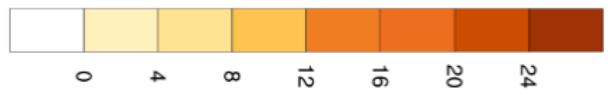

ppb

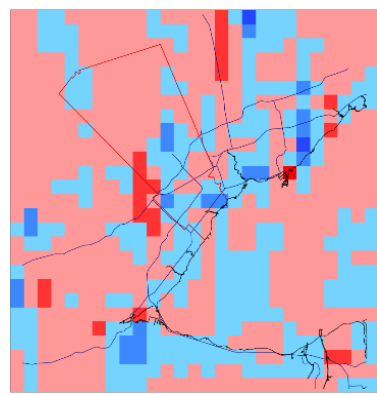

Maximum Difference: $6.1 \mathrm{ppb}$ Minimum Difference: $-5.9 \mathrm{ppb}$

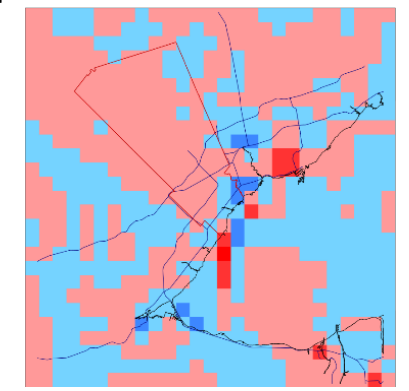

Maximum Difference: $5.1 \mathrm{ppb}$ Minimum Difference: $-4.5 \mathrm{ppb}$

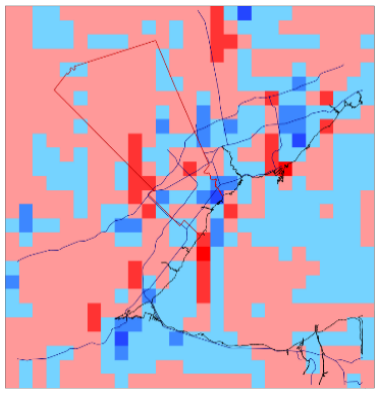

Maximum Difference: $5.1 \mathrm{ppb}$ Minimum Difference: $-4.5 \mathrm{ppb}$

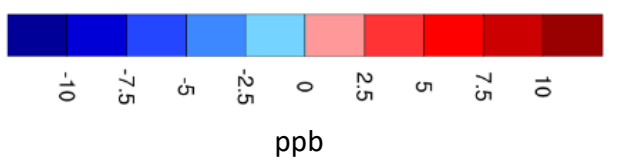

$\mathrm{ppb}$

Figure 5-22. 24-hour average $\mathrm{NO}_{2}$ concentration results from various $4 \mathrm{~km}$ (fine) and $12 \mathrm{~km}$ (coarse) input resolution variation scenarios and the differences between the respective scenario and the all fine $4 \mathrm{~km}$ model run. 

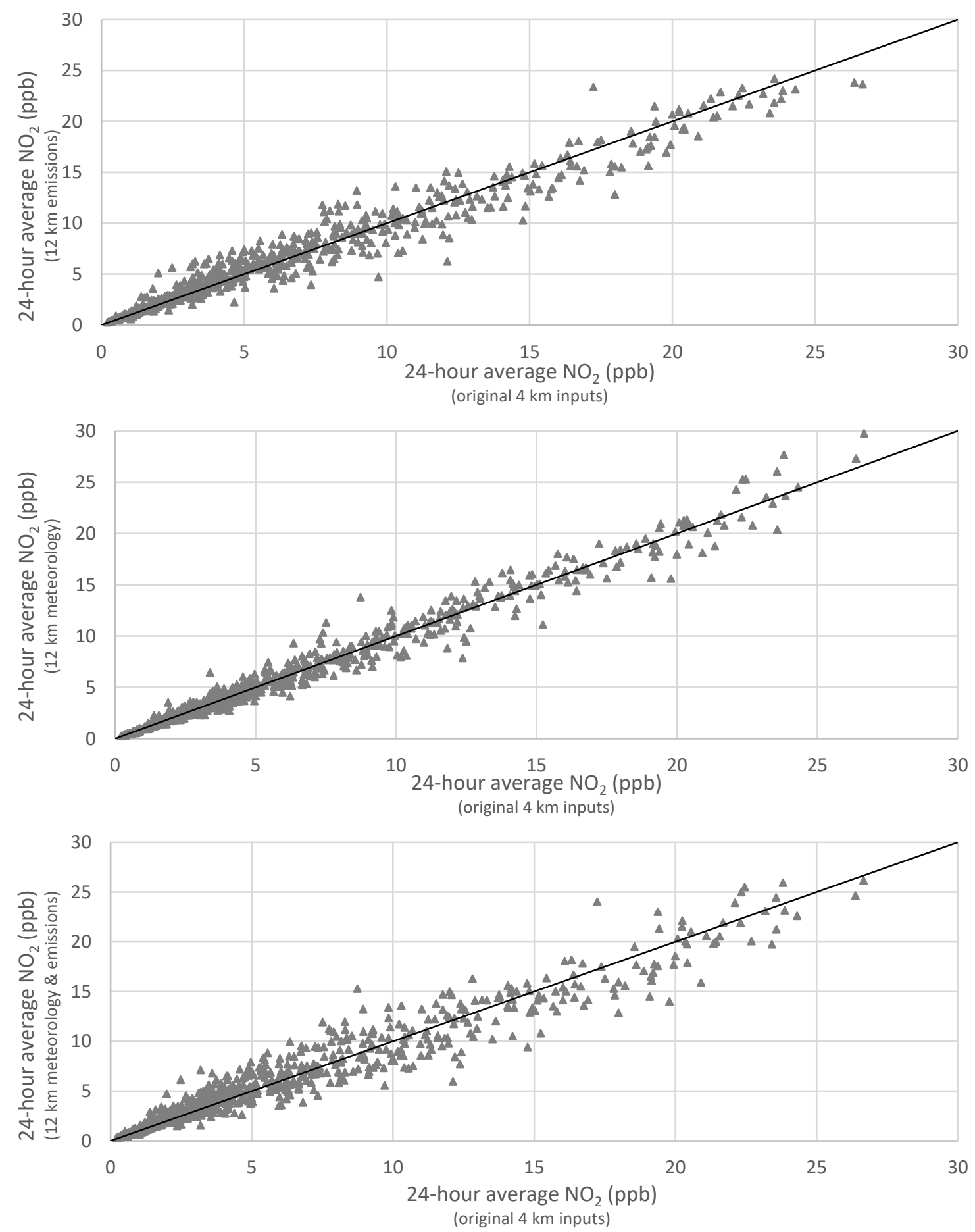

Figure 5-23. Scatter plots comparing the 24-hour average $\mathrm{NO}_{2}$ concentration results for combinations of fine $4 \mathrm{~km}$ resolution with coarse $12 \mathrm{~km}$ inputs: emissions (top), meteorology (middle) and population (bottom). 
The $\mathrm{NO}_{2}$ concentration (daily 24-hour average) results from the $1 \mathrm{~km}$ scenarios with $4 \mathrm{~km}$ input variations, along with each of their respective differences with the original all $1 \mathrm{~km}$ model run, are displayed in Figure 5-24. The scatter comparison plots for daily 24-hour average $\mathrm{NO}_{2}$ concentrations from the $1 \mathrm{~km}$ scenarios with $4 \mathrm{~km}$ input variations are displayed in Figure 5-25.

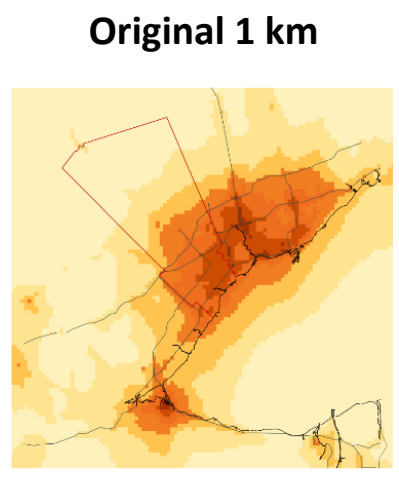

Maximum: $26.8 \mathrm{ppb}$

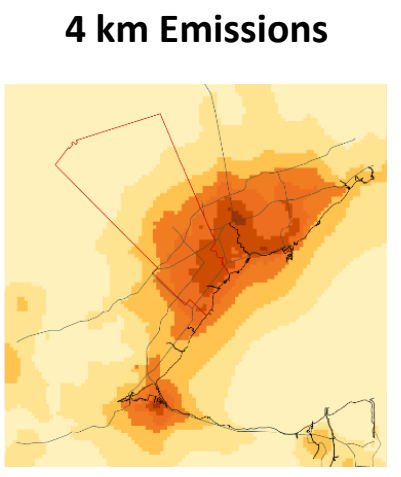

Maximum: $27.2 \mathrm{ppb}$

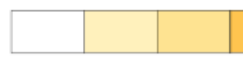

ppb

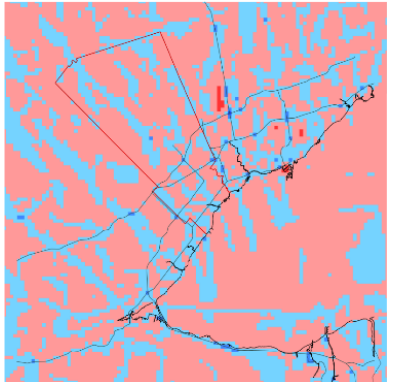

Maximum Difference: $4.3 \mathrm{ppb}$ Minimum Difference: $-5.1 \mathrm{ppb}$
4 km Meteorology

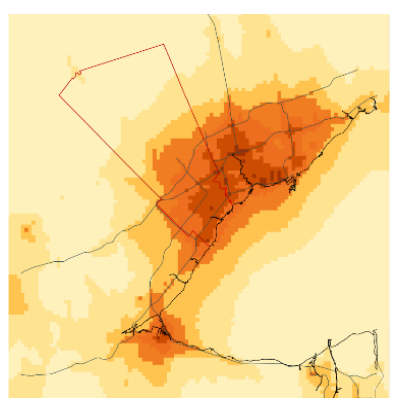

Maximum: $25.9 \mathrm{ppb}$
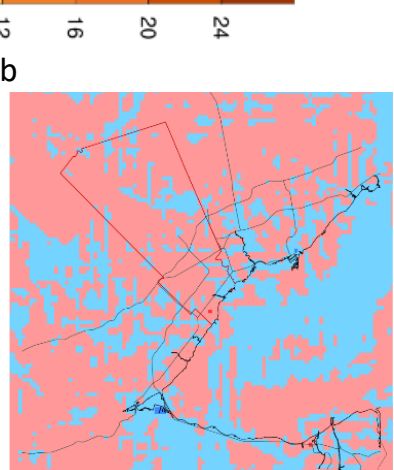

Maximum Difference: $2.7 \mathrm{ppb}$ Minimum Difference: -4.3 ppb

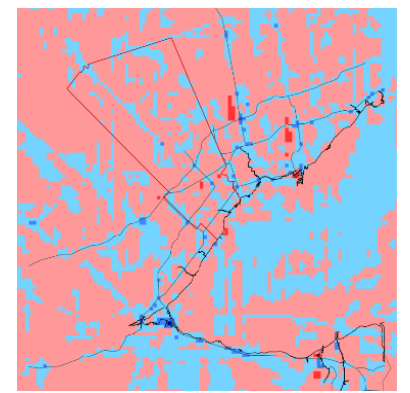

Maximum Difference: $4.2 \mathrm{ppb}$ Minimum Difference: $-7.6 \mathrm{ppb}$

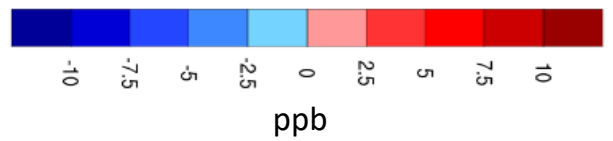

Figure 5-24. 24-hour average $\mathrm{NO}_{2}$ concentration results from various $1 \mathrm{~km}$ (fine) and $4 \mathrm{~km}$ (coarse) input resolution variation scenarios and the differences between the respective scenario and the all fine $1 \mathrm{~km}$ model run. 

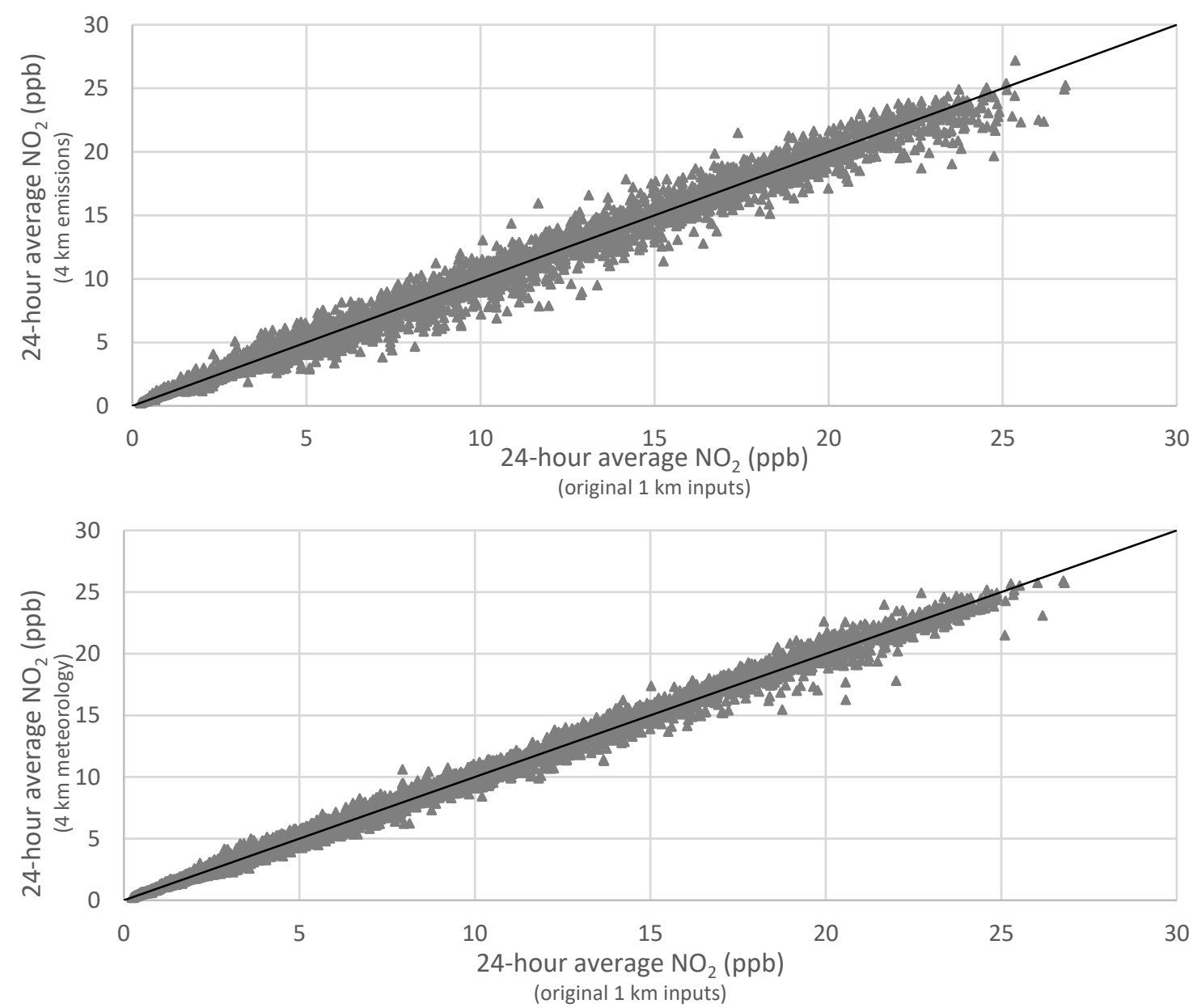

(original 1 km inputs)

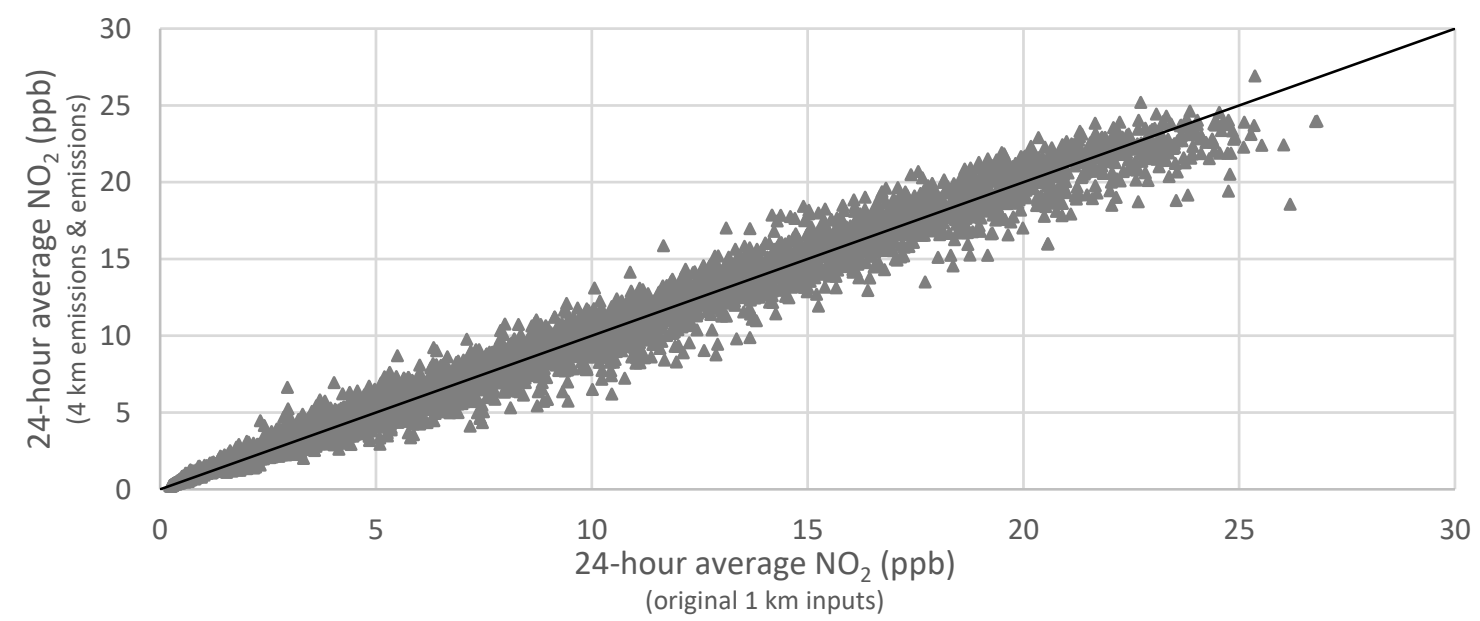

Figure 5-25. Scatter plots comparing the 24-hour average $\mathrm{NO}_{2}$ concentration results for combinations of fine $1 \mathrm{~km}$ resolution with coarse $4 \mathrm{~km}$ inputs: emissions (top), meteorology (middle) and population (bottom). 
The $\mathrm{PM}_{2.5}$ concentration (daily 24-hour average) results from the $12 \mathrm{~km}$ scenarios with $36 \mathrm{~km}$ input variations, along with each of their respective differences with the original all $12 \mathrm{~km}$ model run, are displayed in Figure 5-26. The SCATTER comparison plots for daily 24-hour average $\mathrm{PM}_{2.5}$ concentrations from the $12 \mathrm{~km}$ scenarios with $36 \mathrm{~km}$ input variations are displayed in Figure 5-27.

Original 12 km

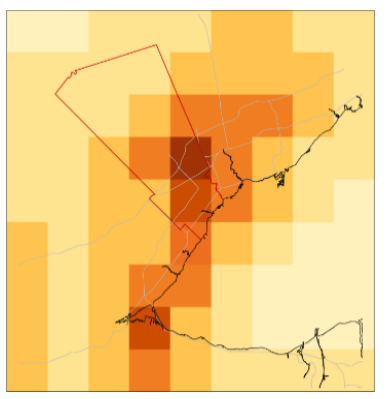

Maximum: $35.4 \mu \mathrm{g} / \mathrm{m}^{3}$
36 km Emissions

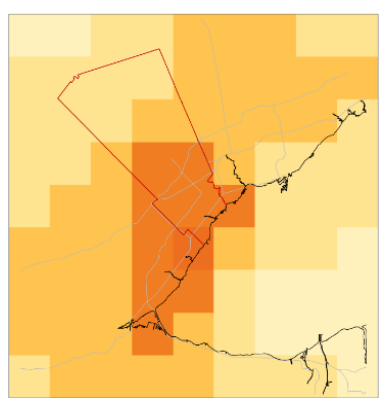

Maximum: $22.8 \mu \mathrm{g} / \mathrm{m}^{3}$
36 km Meteorology

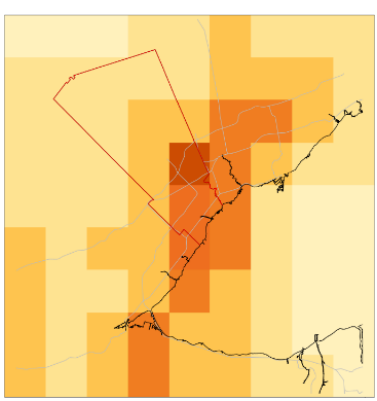

Maximum: $28.4 \mu \mathrm{g} / \mathrm{m}^{3}$
36 km Meteorology

\& Emissions

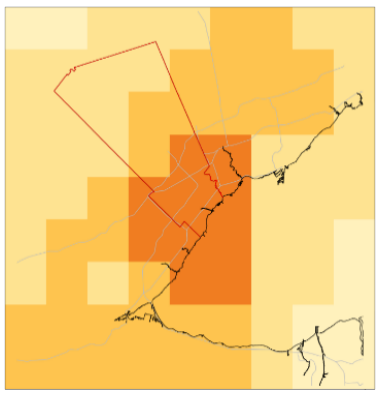

Maximum: $19.3 \mu \mathrm{g} / \mathrm{m}^{3}$

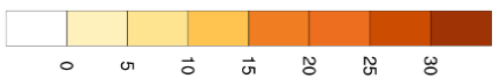

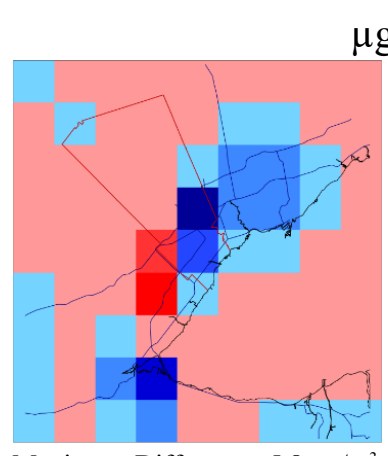

Maximum Difference: $5.8 \mu \mathrm{g} / \mathrm{m}^{3}$ Min. Difference: $-16.0 \mu \mathrm{g} / \mathrm{m}^{3}$ $\mu \mathrm{g} / \mathrm{m}^{3}$

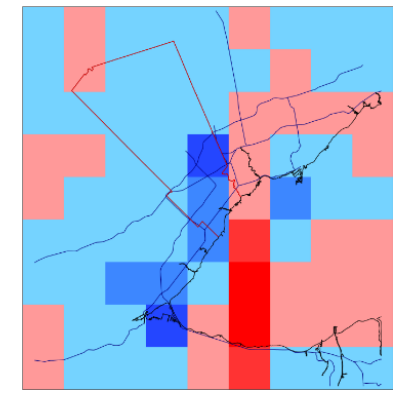

Maximum Difference: $5.5 \mu \mathrm{g} / \mathrm{m}^{3}$ Minimum Difference: $-7.1 \mu \mathrm{g} / \mathrm{m}^{3}$

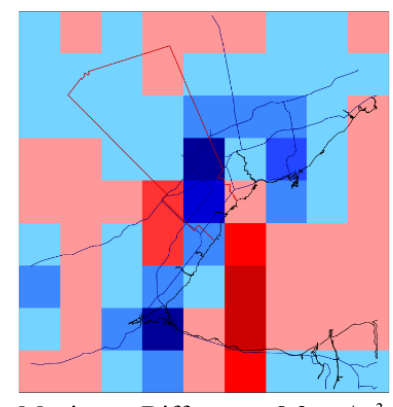

Maximum Difference: $8.3 \mu \mathrm{g} / \mathrm{m}^{3}$ Min. Difference: $-19.6 \mu \mathrm{g} / \mathrm{m}^{3}$

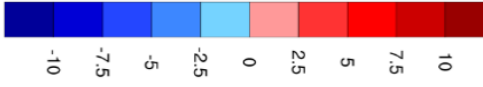

$\mu \mathrm{g} / \mathrm{m}^{3}$

Figure 5-26. 24-hour average $\mathrm{PM}_{2.5}$ concentration results from various $12 \mathrm{~km}$ (fine) and $36 \mathrm{~km}$ (coarse) input resolution variation scenarios and the differences between the respective scenario and the all fine $12 \mathrm{~km}$ model run. 

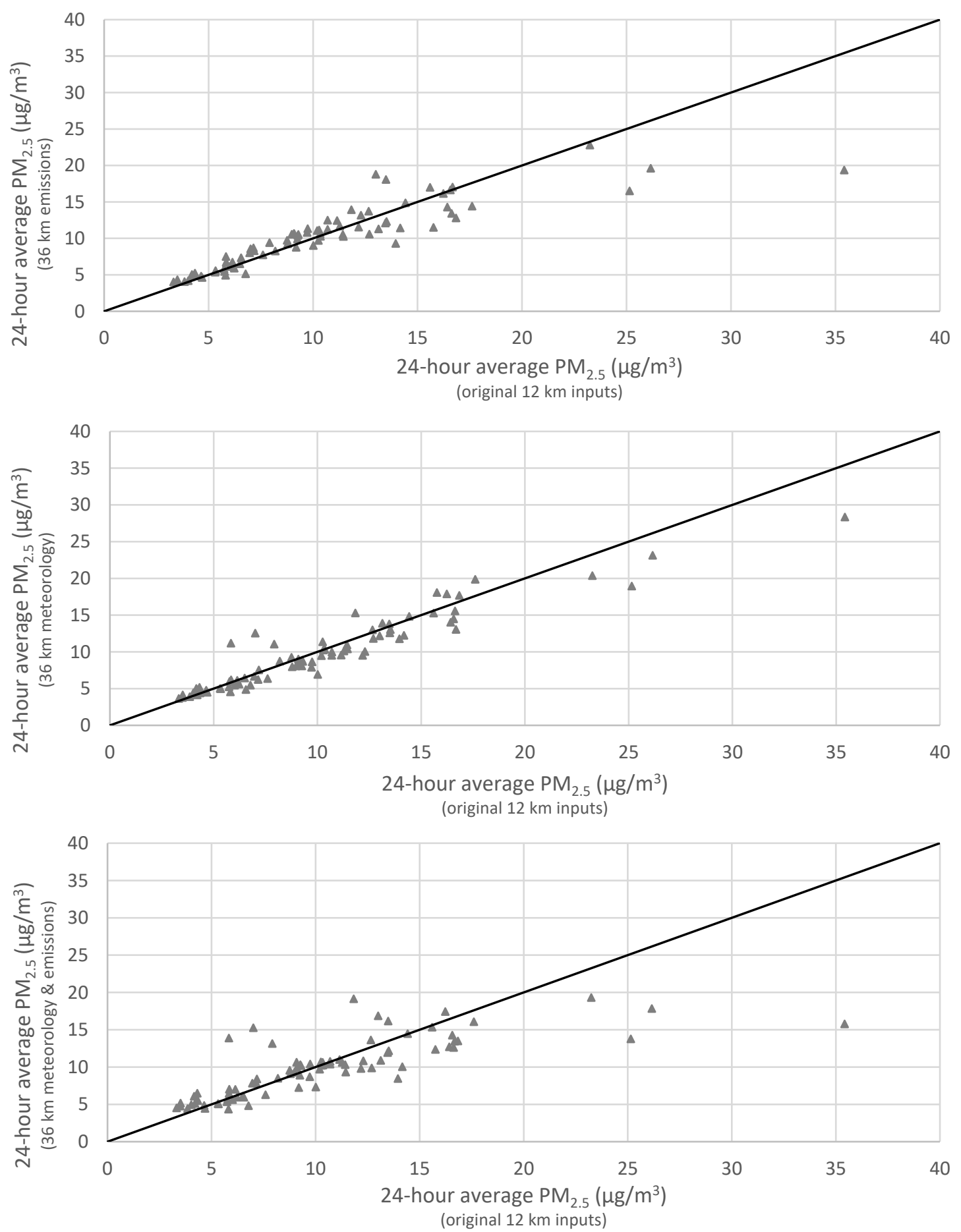

Figure 5-27. Scatter plots comparing the 24-hour average $\mathrm{PM}_{2.5}$ concentration results for combinations of fine 12 $\mathrm{km}$ resolution with coarse $36 \mathrm{~km}$ inputs: emissions (top), meteorology (middle) and population (bottom). 
The $\mathrm{PM}_{2.5}$ concentration (daily 24-hour average) results from the $4 \mathrm{~km}$ scenarios with $12 \mathrm{~km}$ input variations, along with each of their respective differences with the original all $4 \mathrm{~km}$ model run, are displayed in Figure 5-28. The scatter comparison plots for daily 24-hour average $\mathrm{PM}_{2.5}$ concentrations from the $4 \mathrm{~km}$ scenarios with $12 \mathrm{~km}$ input variations are displayed in Figure 5-

29.

Original 4 km

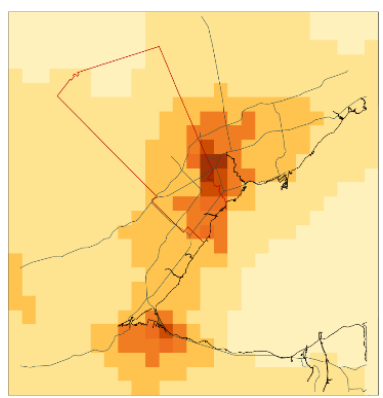

Maximum:82.9 $\mu \mathrm{g} / \mathrm{m}^{3}$
12 km Emissions

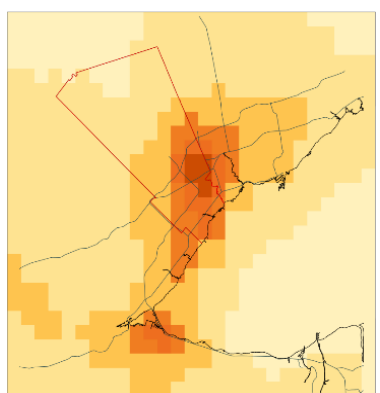

Maximum: $35.2 \mu \mathrm{g} / \mathrm{m}^{3}$
12 km Meteorology

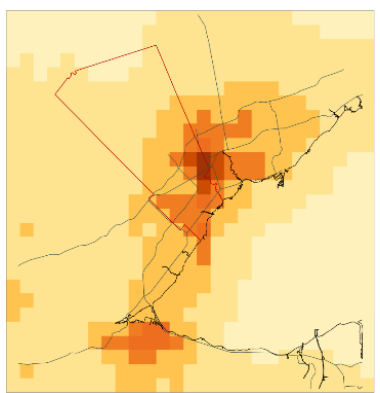

Maximum: $91.9 \mu \mathrm{g} / \mathrm{m}^{3}$

\section{$12 \mathrm{~km}$ Meteorology \& Emissions}

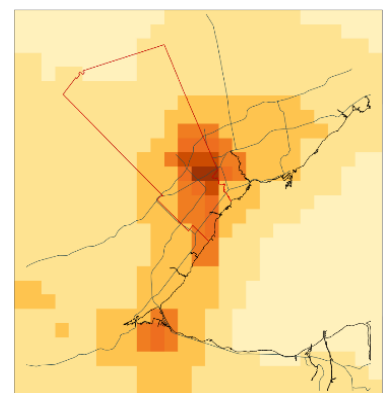

Maximum: $38.5 \mu \mathrm{g} / \mathrm{m}^{3}$

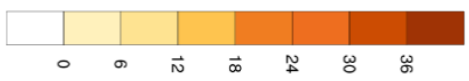

$$
\mu \mathrm{g} / \mathrm{m}^{3}
$$

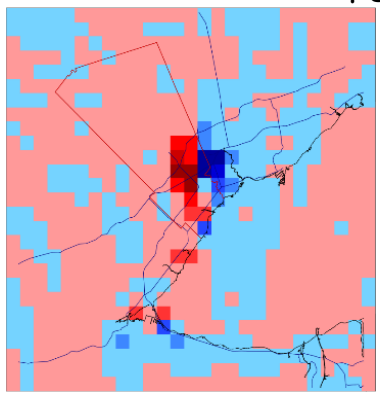

Maximum Difference: 16.3 $\mu \mathrm{g} / \mathrm{m}^{3}$ Minimum Difference: -50.8 $\mu \mathrm{g} / \mathrm{m}^{3}$

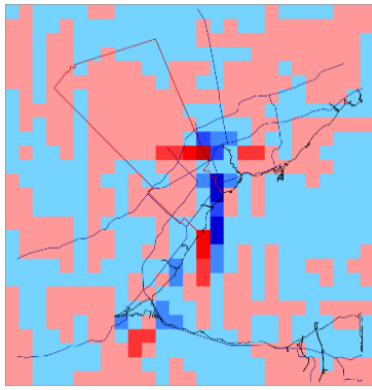

Maximum Difference: $9.0 \mu \mathrm{g} / \mathrm{m}^{3}$ Minimum Difference: -9.4 $\mu \mathrm{g} / \mathrm{m}^{3}$

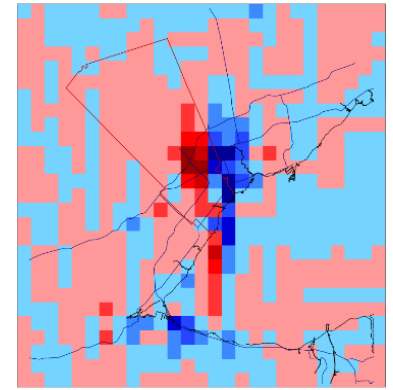

Maximum Difference: 21.1 $\mu \mathrm{g} / \mathrm{m}^{3}$ Minimum Difference: -48.9 $\mu \mathrm{g} / \mathrm{m}^{3}$

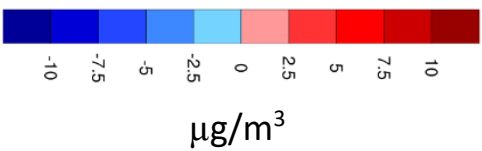

Figure 5-28. 24-hour average $\mathrm{PM}_{2.5}$ concentration results from various $4 \mathrm{~km}$ (fine) and $12 \mathrm{~km}$ (coarse) input resolution variation scenarios and the differences between the respective scenario and the all fine $4 \mathrm{~km}$ model run. 

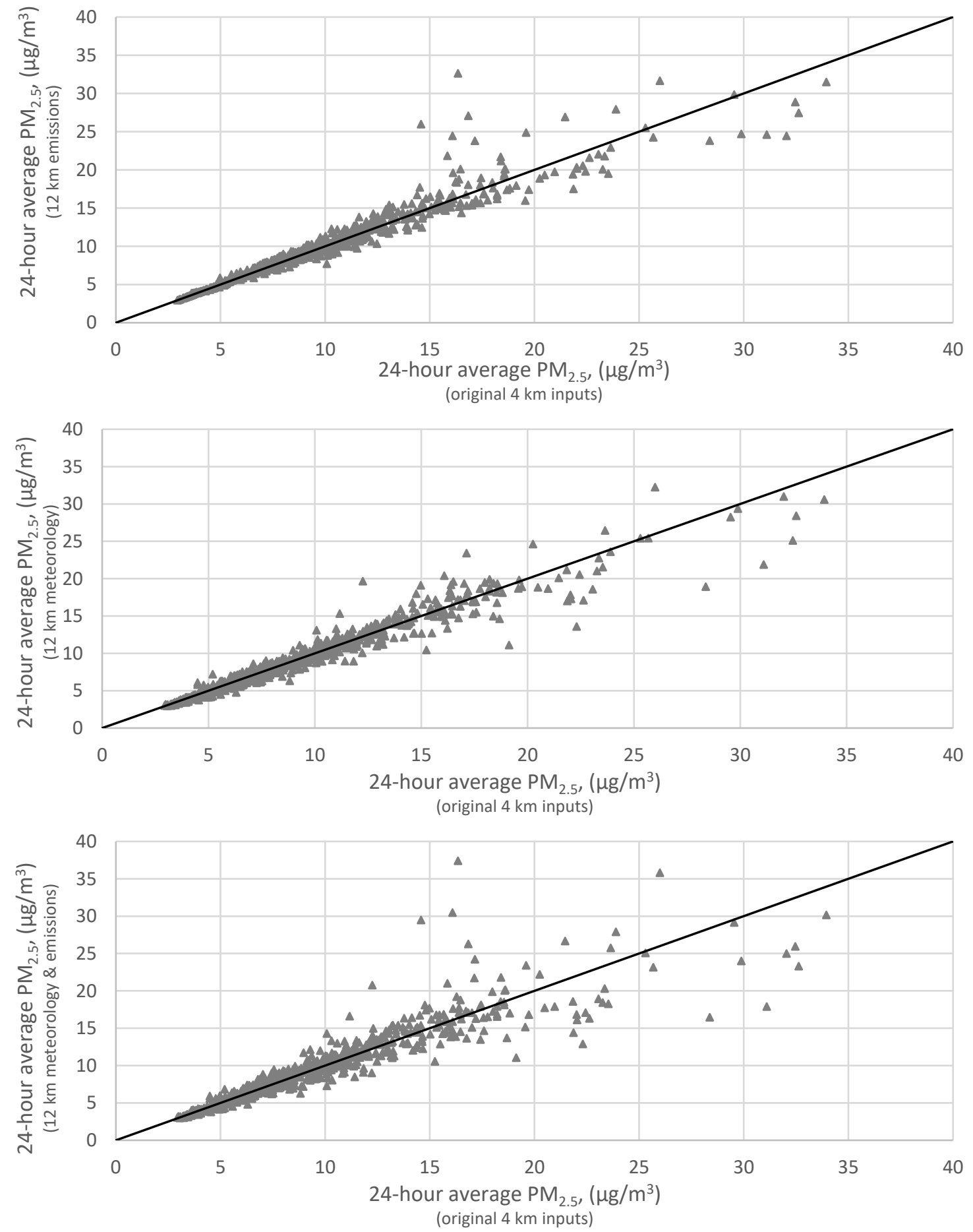

Figure 5-29. scatter plots comparing the 24-hour average $\mathrm{PM}_{2.5}$ concentration results for combinations of fine $4 \mathrm{~km}$ resolution with coarse $12 \mathrm{~km}$ inputs: emissions (top), meteorology (middle) and population (bottom). 
The $\mathrm{PM}_{2.5}$ concentration (daily 24-hour average) results from the $1 \mathrm{~km}$ scenarios with $4 \mathrm{~km}$ input variations, along with each of their respective differences with the original all $4 \mathrm{~km}$ model run, are displayed in Figure 5-30. The scatter comparison plots for daily 24-hour average $\mathrm{PM}_{2.5}$ concentrations from the $1 \mathrm{~km}$ scenarios with $4 \mathrm{~km}$ input variations are displayed in Figure 5-31.

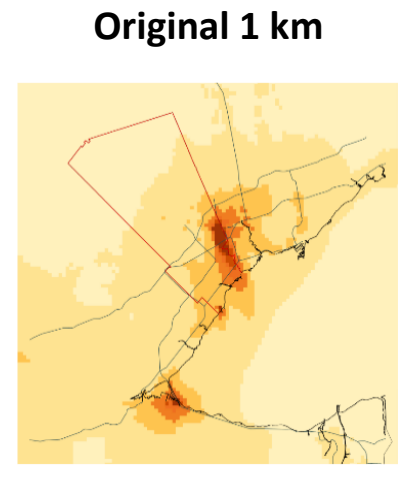

Maximum: $288.9 \mu \mathrm{g} / \mathrm{m}^{3}$

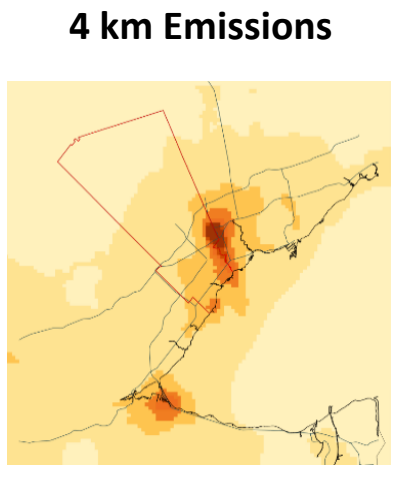

Maximum: $70.1 \mu \mathrm{g} / \mathrm{m}^{3}$

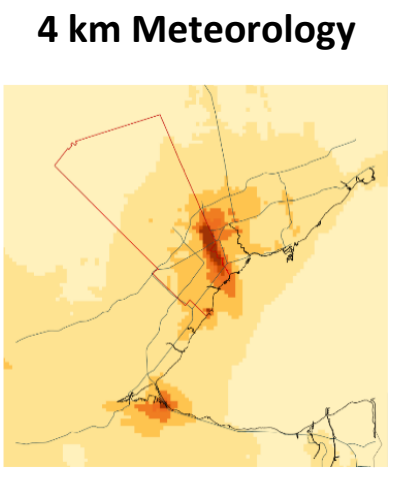

Maximum: $275.0 \mu \mathrm{g} / \mathrm{m}^{3}$
$4 \mathrm{~km}$ Meteorology \& Emissions

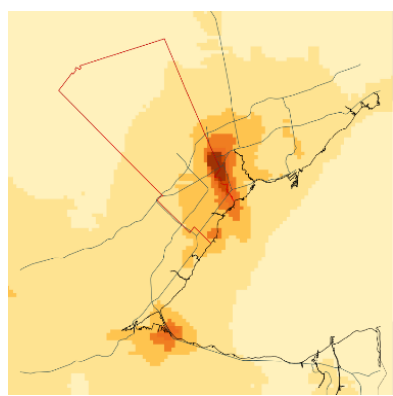

Maximum: $85.7 \mu \mathrm{g} / \mathrm{m}^{3}$

Difference with Original:

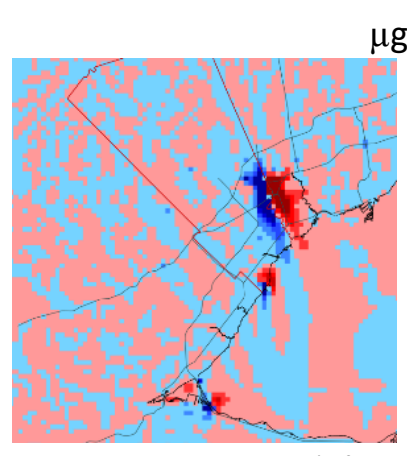

Max. Difference: $42.2 \mu \mathrm{g} / \mathrm{m}^{3}$ Min. Difference: $-235.3 \mu \mathrm{g} / \mathrm{m}^{3}$

$\mu \mathrm{g} / \mathrm{m}^{3}$

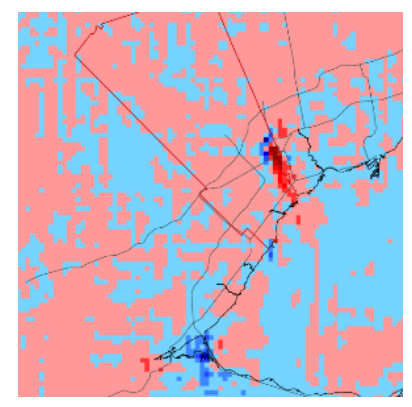

Max. Difference: $13.6 \mu \mathrm{g} / \mathrm{m}^{3}$ Min. Difference: $-13.9 \mu \mathrm{g} / \mathrm{m}^{3}$

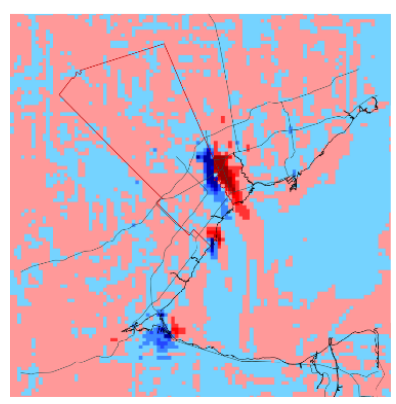

Max. Difference: $47.4 \mu \mathrm{g} / \mathrm{m}^{3}$ Min. Difference: $-232.7 \mu \mathrm{g} / \mathrm{m}^{3}$

$\mu \mathrm{g} / \mathrm{m}^{3}$

Figure 5-30. 24-hour average $\mathrm{PM}_{2.5}$ concentration results from various $1 \mathrm{~km}$ (fine) and $4 \mathrm{~km}$ (coarse) input resolution variation scenarios and the differences between the respective scenario and the all fine $1 \mathrm{~km}$ model run. 

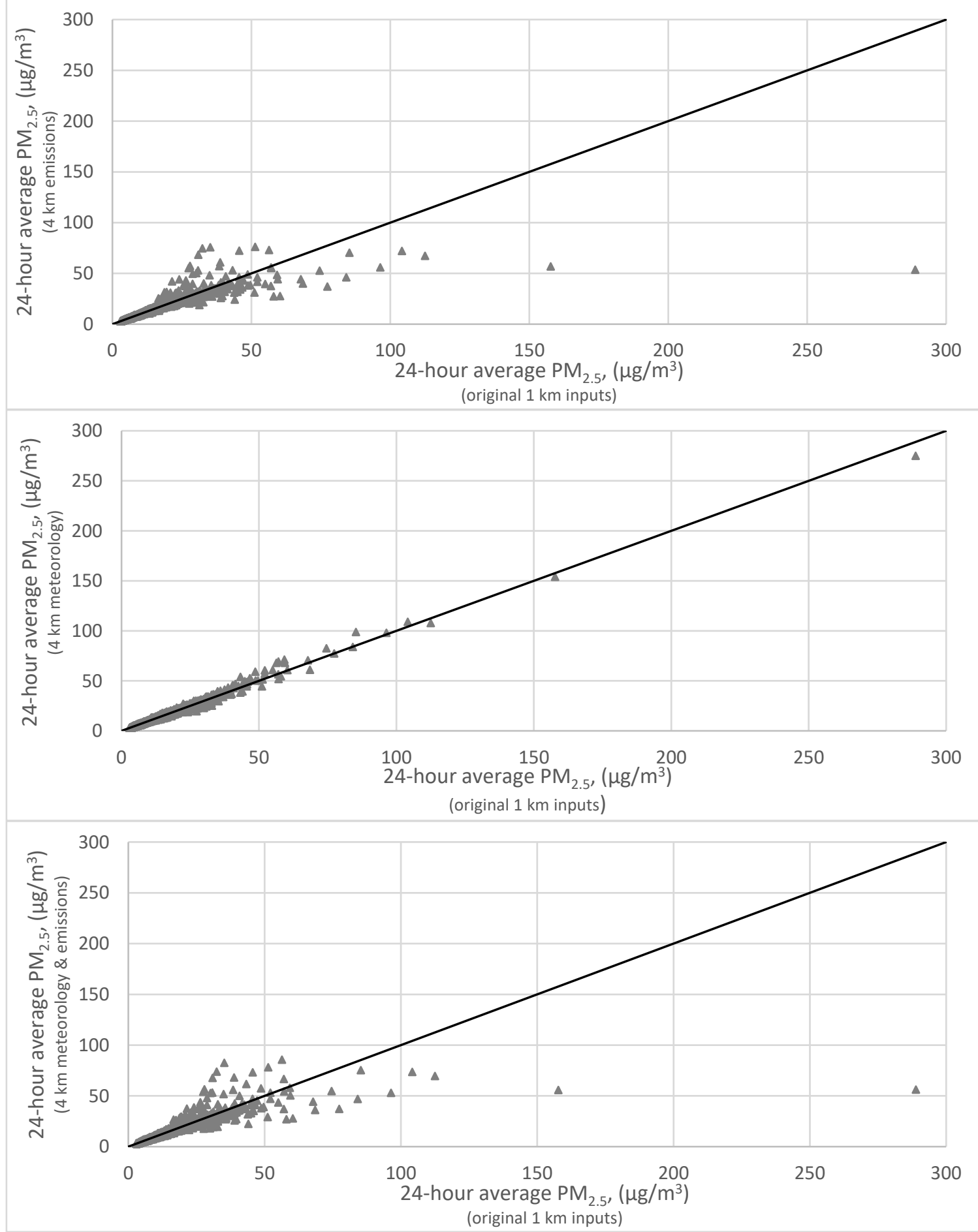

Figure 5-31. Scatter plots comparing the 24-hour average $\mathrm{PM}_{2.5}$ concentration results for combinations of fine $1 \mathrm{~km}$ resolution with coarse $4 \mathrm{~km}$ inputs: emissions (top), meteorology (middle) and both meteorology and emissions (bottom). 
The chronic health impacts, parameterized by monetized mortality, due to chronic exposure to $\mathrm{O}_{3}$ for the 12 and $36 \mathrm{~km}$ resolution scenarios are displayed in Figure 5-32 for each input parameter, and in Figure 5-33 as scatter plots with the coarse $36 \mathrm{~km}$ input results on the $y$-axis and the original fine $12 \mathrm{~km}$ input results on the $\mathrm{x}$-axis.

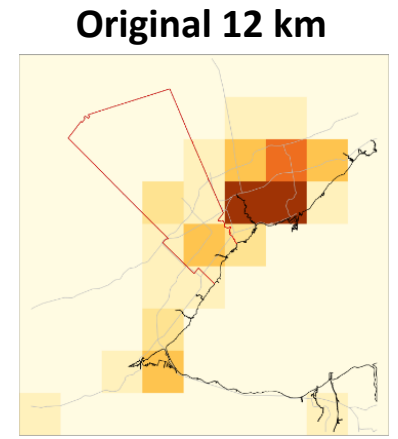

Maximum: $\$ 5.78 \mathrm{~B}$

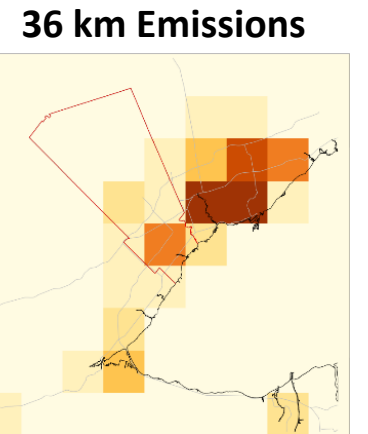

Maximum: $\$ 6.17 \mathrm{~B}$
36 km Meteorology

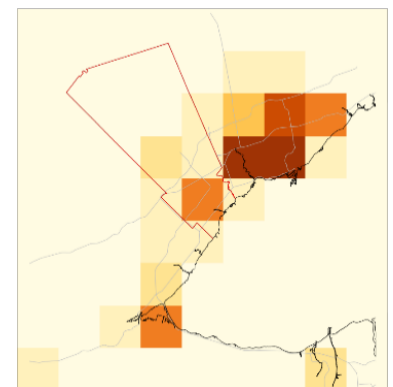

Maximum: $\$ 5.76 \mathrm{~B}$
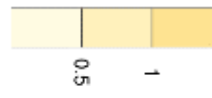

$\$ 10^{9}$

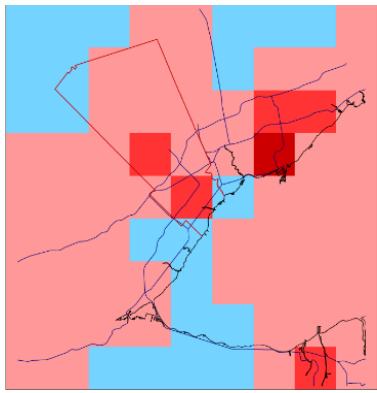

Maximum Difference: $\$ 0.380 \mathrm{~B}$ Minimum Difference: $-\$ 0.04 \mathrm{~B}$

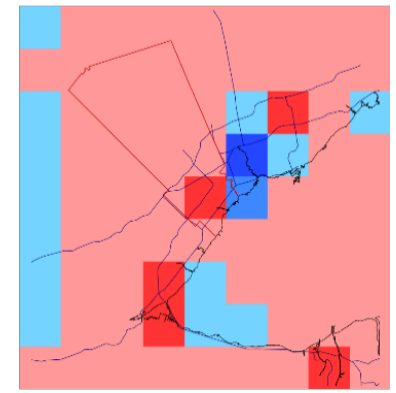

Maximum Difference: $\$ 0.149 B$ Minimum Difference: $\$ 0.276 \mathrm{~B}$

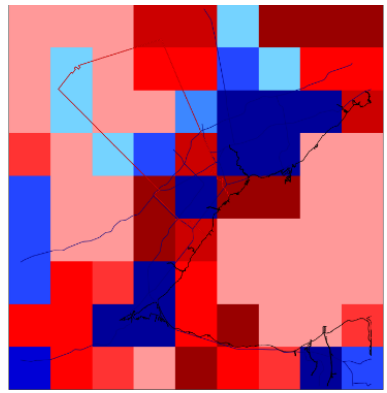

Maximum Difference: $\$ 0.854 \mathrm{~B}$ Minimum Difference: $-\$ 5.06 \mathrm{~B}$

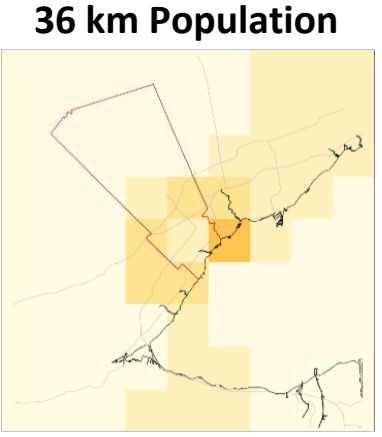

Maximum: $\$ 1.83 \mathrm{~B}$
Difference with

Original:

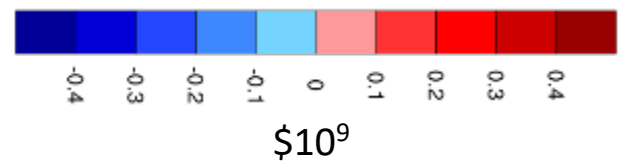

Figure 5-32 Monetized health impacts due to Chronic $\mathrm{O}_{3}$ exposure from various $12 \mathrm{~km}$ (fine) and $36 \mathrm{~km}$ (coarse) input resolution scenarios and the differences between the respective scenario and the all fine $12 \mathrm{~km}$ model run. 


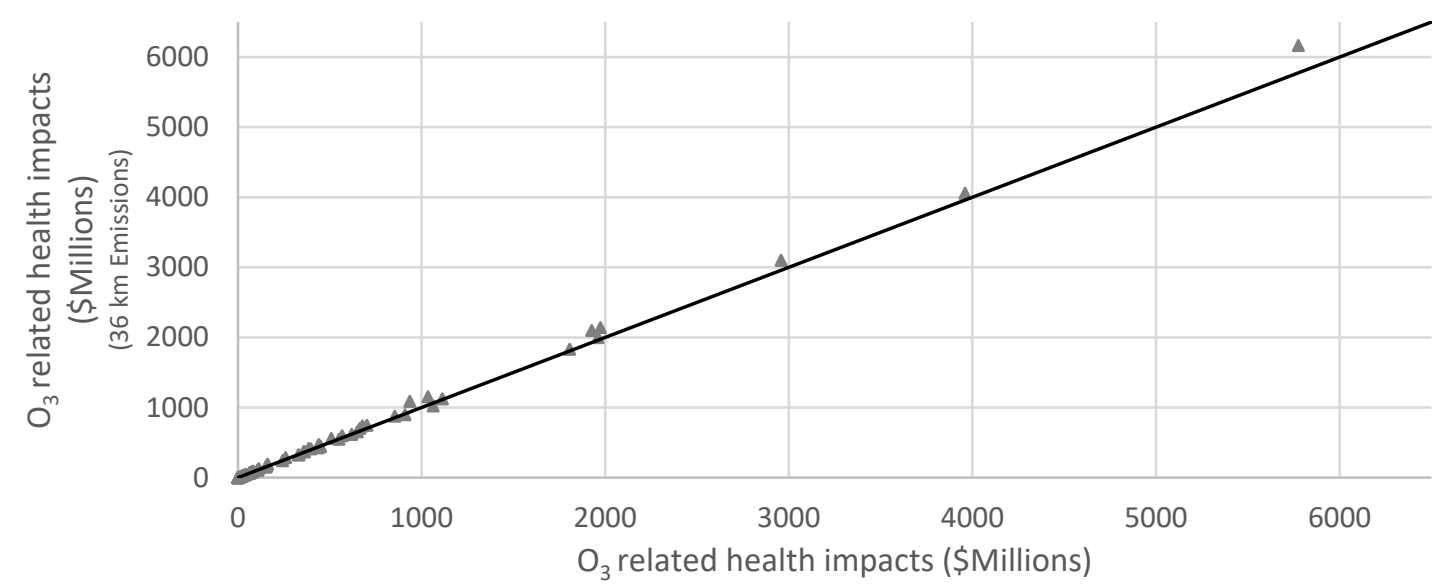

(Original $12 \mathrm{~km}$ input)

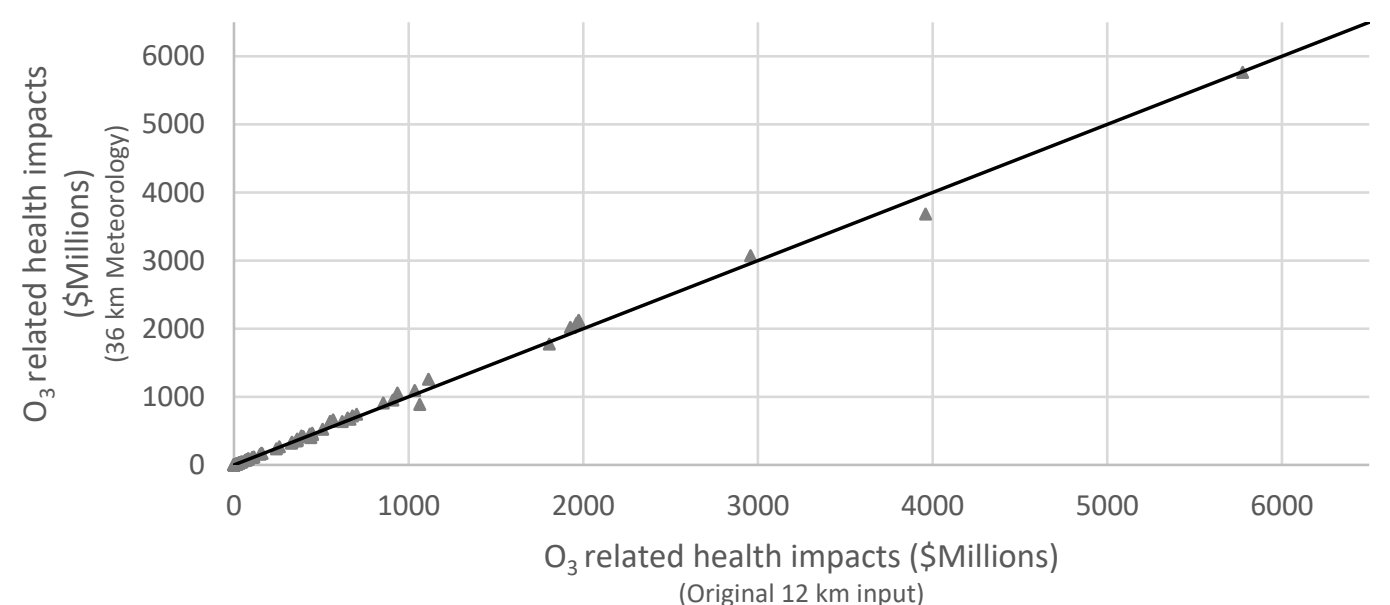

(Original $12 \mathrm{~km}$ input)

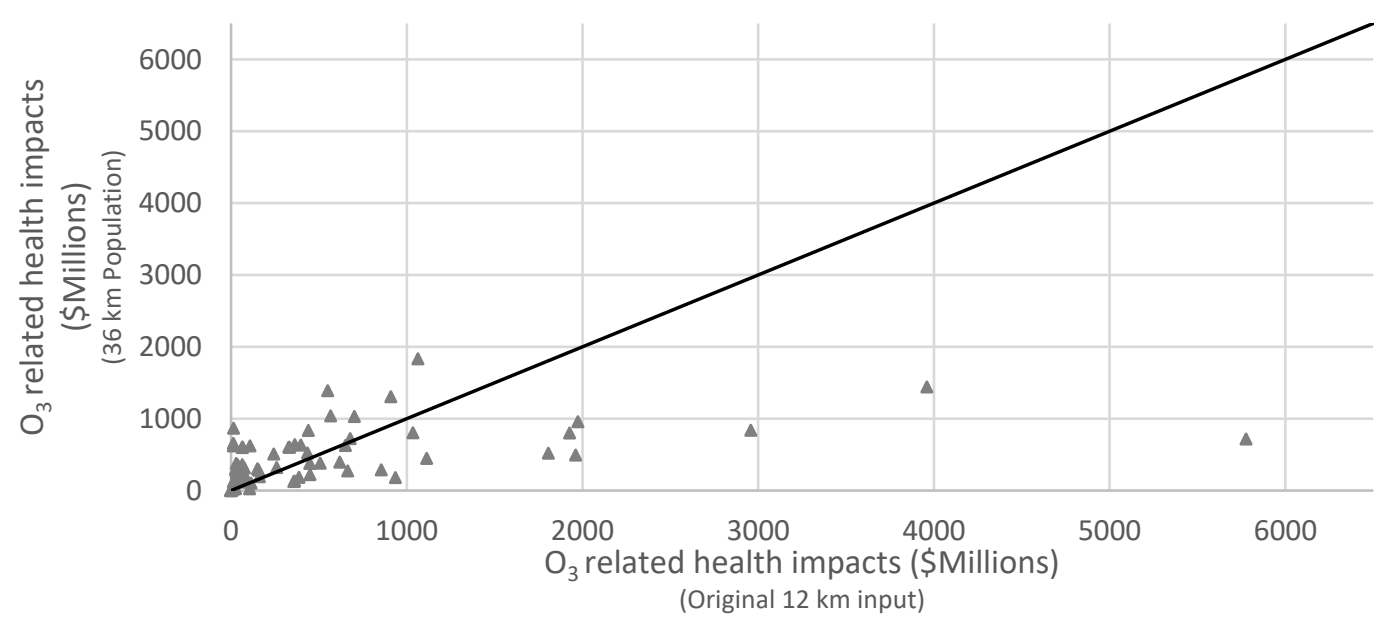

Figure 5-33. Scatter plots comparing the monetized health impacts due to chronic $\mathrm{O}_{3}$ exposure results for combinations of fine $12 \mathrm{~km}$ resolution with coarse $36 \mathrm{~km}$ inputs: emissions (top), meteorology (middle), and population (bottom). 
The chronic health impacts, parameterized by monetized mortality, due to chronic exposure to $\mathrm{O}_{3}$ for the 4 and $12 \mathrm{~km}$ resolution scenarios are displayed in Figure 5-34 for each input parameter, and in Figure 5-35 as scatter plots with the coarse $12 \mathrm{~km}$ input results on the $y$-axis and the original fine $4 \mathrm{~km}$ input results on the $\mathrm{x}$-axis.

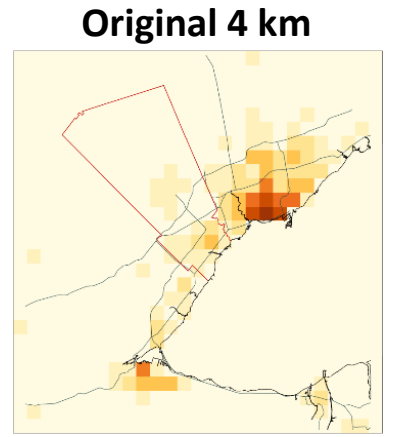

Maximum: \$1.11B

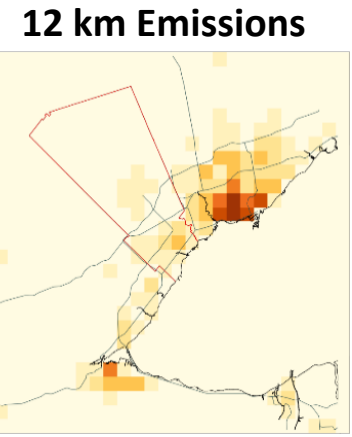

Maximum: \$1.18B
12 km Meteorology

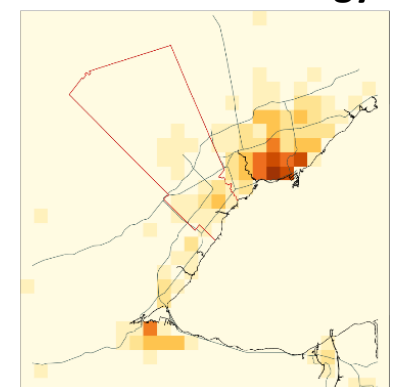

Maximum: \$1.09B

\section{$12 \mathrm{~km}$ Population}

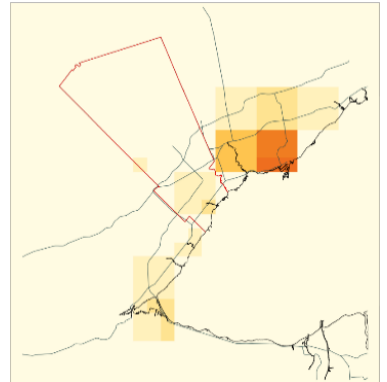

Maximum: $\$ 0.64 \mathrm{~B}$

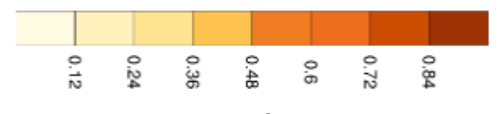

$\$ 10^{6}$

Difference with Original:

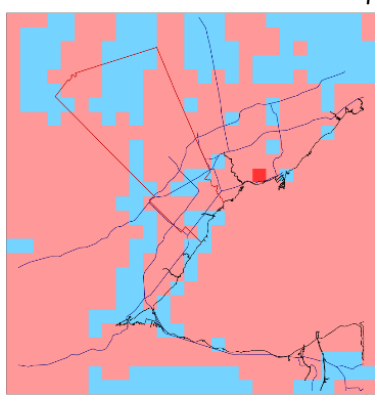

Max. Difference: $\$ 70.3 \mathrm{M}$ Min. Difference: $-\$ 3.84 \mathrm{M}$

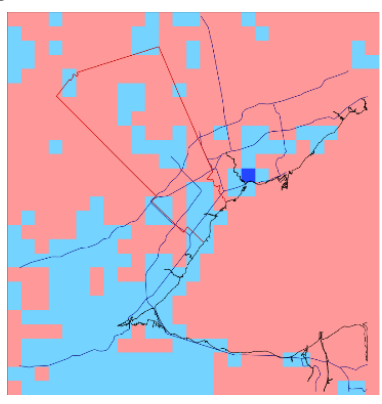

Max. Difference: $\$ 2.56 \mathrm{M}$ Min. Difference: $-\$ 101.8 \mathrm{M}$

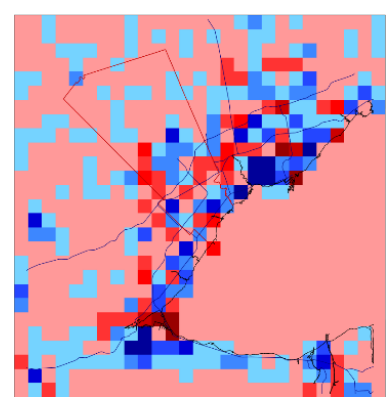

Max. Difference: $\$ 557.6 \mathrm{M}$ Min. Difference: $-\$ 486.3 \mathrm{M}$

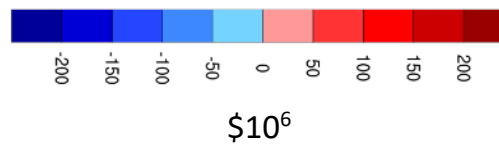

Figure 5-34: Monetized health impacts due to chronic $\mathrm{O}_{3}$ exposure from various $4 \mathrm{~km}$ (fine) and $12 \mathrm{~km}$ (coarse) input resolution scenarios and the differences between the respective scenario and the all fine $4 \mathrm{~km}$ model run. 

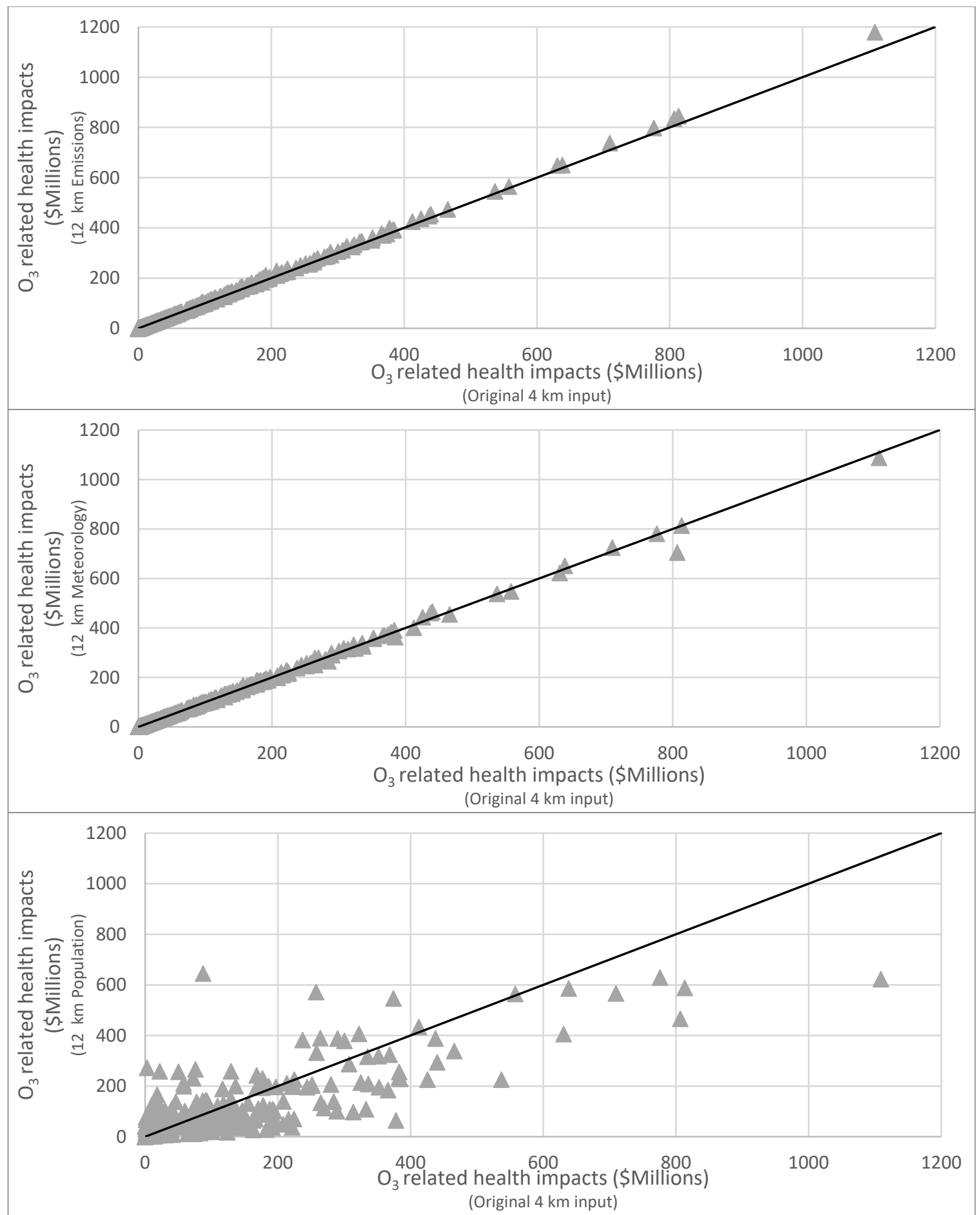

Figure 5-35. Scatter plots comparing the monetized health impacts due to chronic $\mathrm{O}_{3}$ exposure results for combinations of fine $4 \mathrm{~km}$ resolution with coarse $12 \mathrm{~km}$ inputs: emissions (top), meteorology (middle), and population (bottom). 
The chronic health impacts, parameterized by monetized mortality, due to chronic exposure to $\mathrm{O}_{3}$ for the 1 and $4 \mathrm{~km}$ resolution scenarios are displayed in Figure 5-36 for each input parameter, and in Figure 5-37 as scatter plots with the coarse $4 \mathrm{~km}$ input results on the $\mathrm{y}$-axis and the original fine $1 \mathrm{~km}$ input results on the $\mathrm{x}$-axis.

Original $1 \mathrm{~km}$

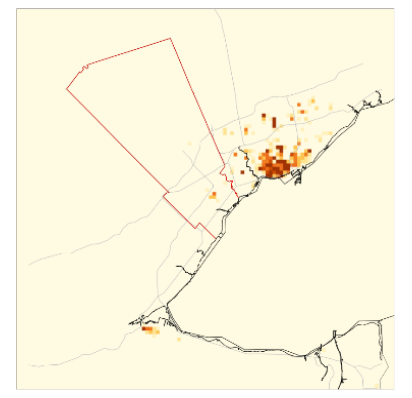

Maximum: \$159.2M

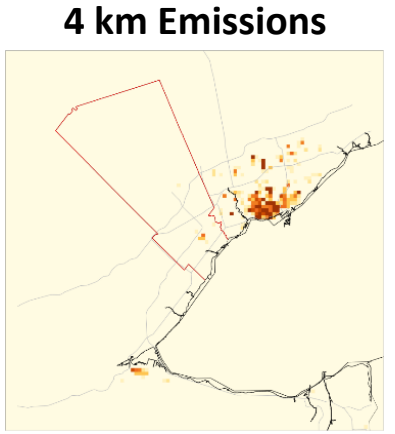

Maximum: \$164.9M
4 km Meteorology

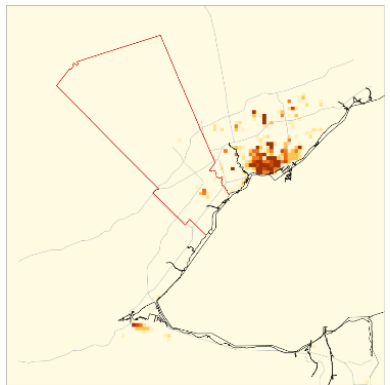

Maximum: \$165.7M

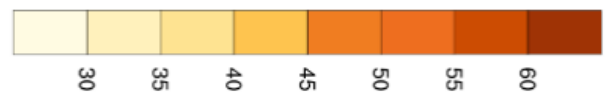

$\$ 10^{6} /$ year
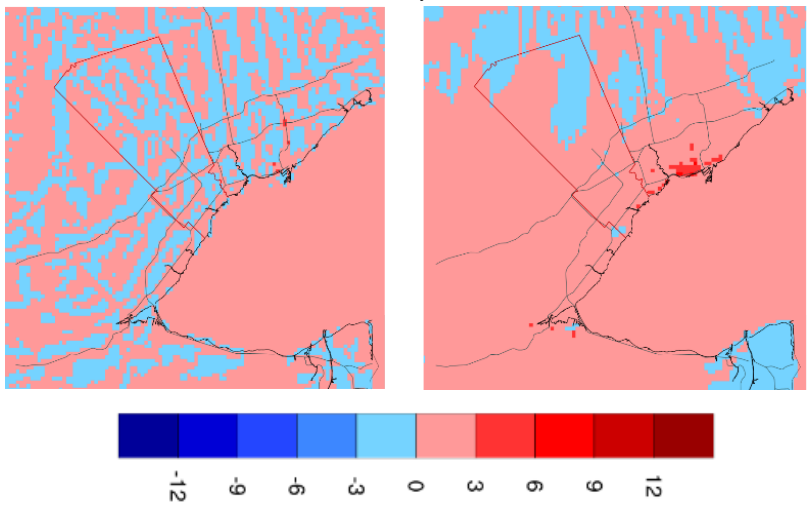

$\$ 10^{6} /$ year

Max. Difference: $\$ 5.66 \mathrm{M}$ Min. Difference: $-\$ 2.09 \mathrm{M}$
Max. Difference: $\$ 6.91 \mathrm{M}$

Min. Difference: $-\$ 1.44 \mathrm{M}$
4 km Population

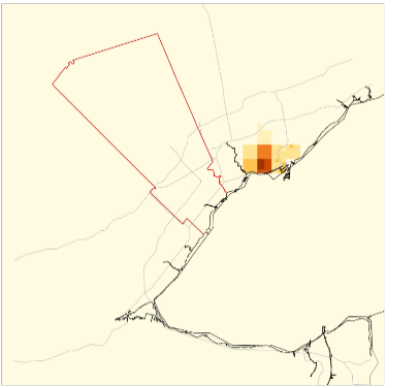

Maximum: $\$ 61.6 \mathrm{M}$

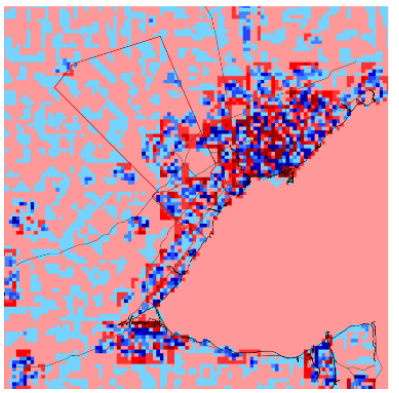

Max. Difference: $\$ 41.3 \mathrm{M}$

Min. Difference: $-\$ 119.4 \mathrm{M}$

Figure 5-36 Monetized annual health impacts due to chronic $\mathrm{O}_{3}$ exposure from various $1 \mathrm{~km}$ (fine) and $4 \mathrm{~km}$ (coarse) input resolution scenarios and the differences between the respective scenario and the all fine $1 \mathrm{~km}$ model run. 

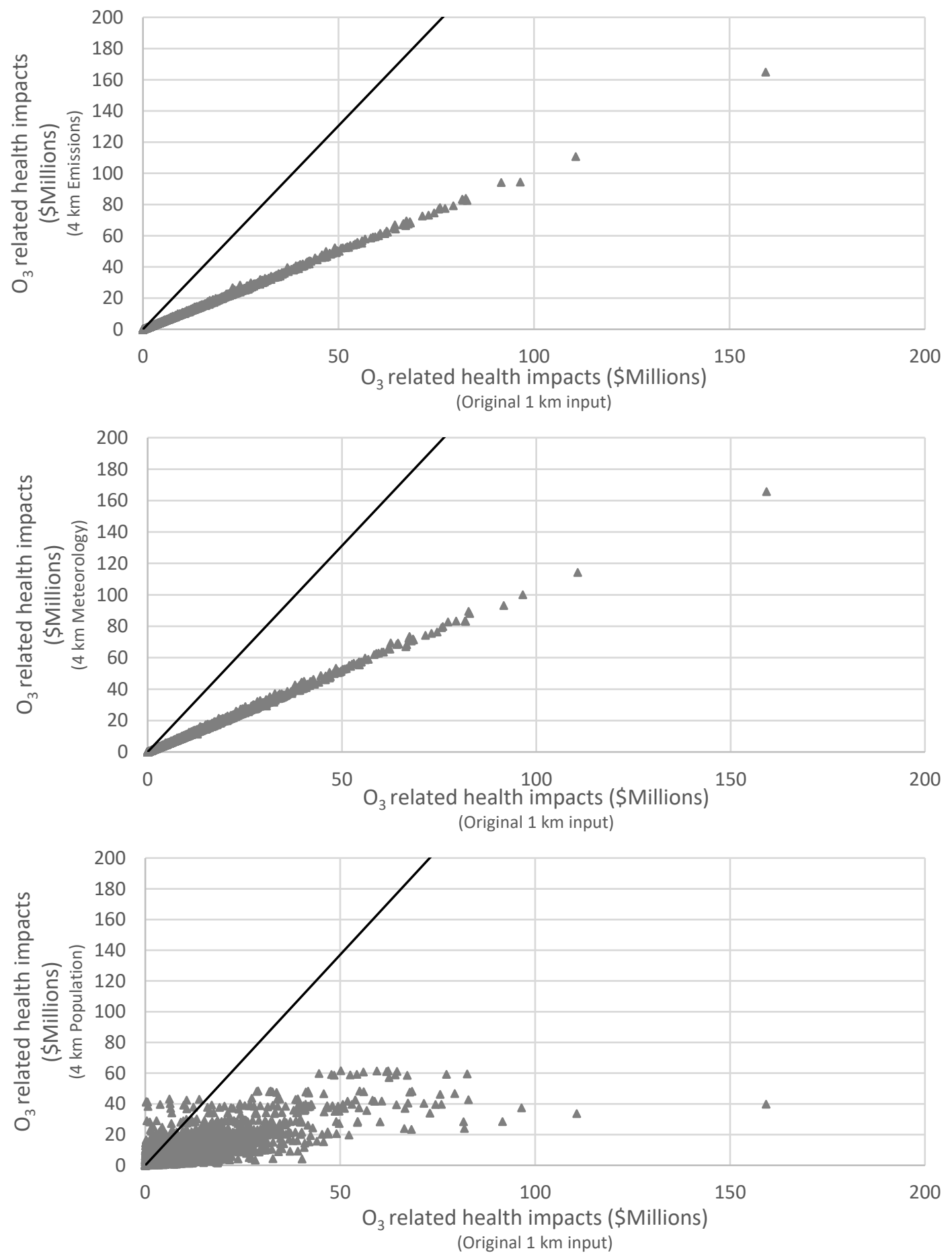

Figure 5-37. scatter plots comparing the monetized health impacts due to chronic $\mathrm{O}_{3}$ exposure results for combinations of fine $1 \mathrm{~km}$ resolution with coarse $4 \mathrm{~km}$ inputs: emissions (top), meteorology (middle), and population (bottom). 
The chronic health impacts, parameterized by monetized mortality, due to chronic exposure to $\mathrm{NO}_{2}$ for the 12 and $36 \mathrm{~km}$ resolution scenarios are displayed in Figure 5-38 for each input parameter, and in Figure 5-39 as scatter plots with the coarse $36 \mathrm{~km}$ input results on the $y$-axis and the original fine $12 \mathrm{~km}$ input results on the $\mathrm{x}$-axis.

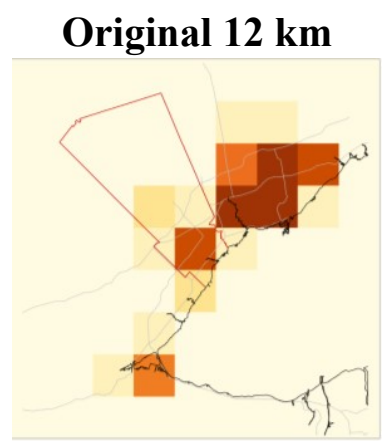

Maximum: \$4.96B

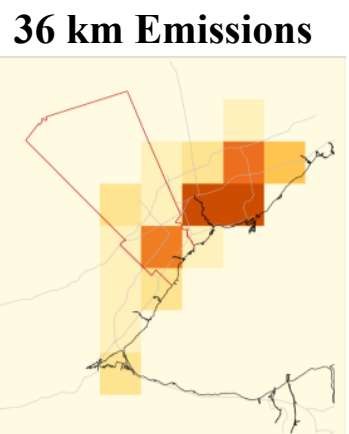

Maximum: \$2.09B

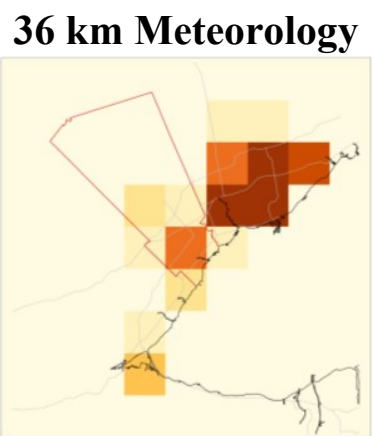

Maximum: \$4.22B
36 km Population

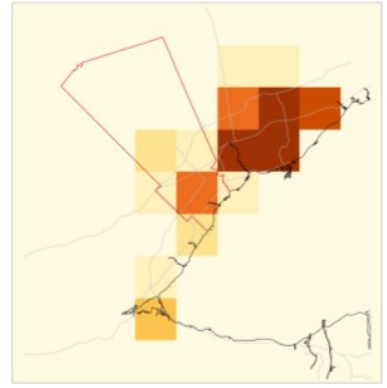

Maximum: \$1.02B

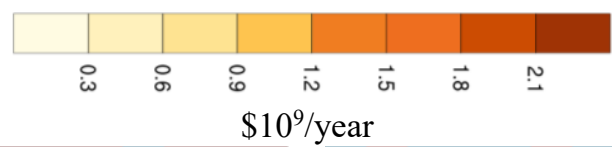

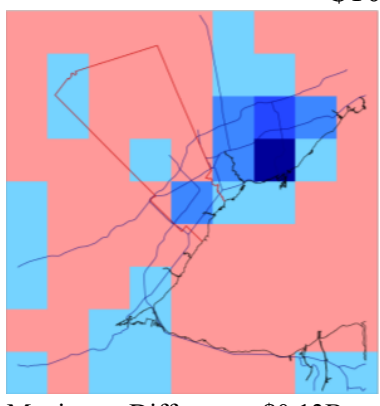

Maximum Difference: \$0.13B Minimum Difference: $-\$ 2.87 \mathrm{~B}$

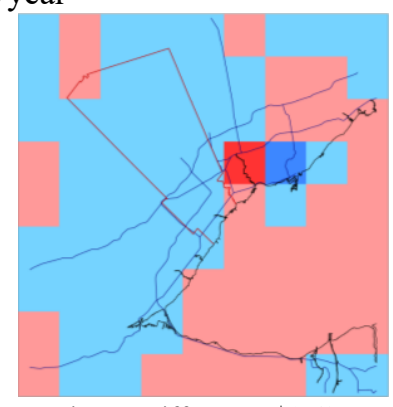

Maximum Difference: \$0.62B Minimum Difference: $-\$ 7.32 \mathrm{~B}$

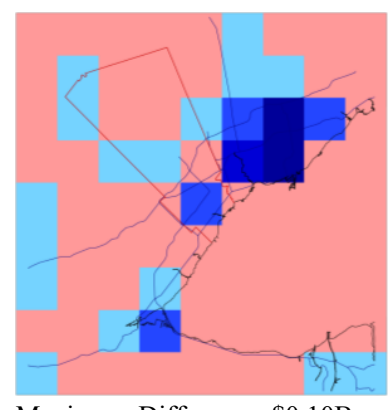

Maximum Difference: $\$ 0.10 \mathrm{~B}$ Minimum Difference: $-\$ 3.04 B$

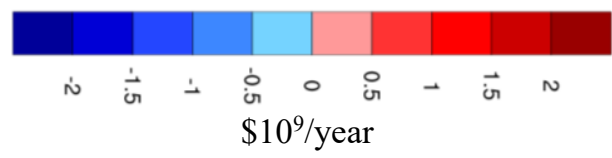

Figure 5-38. Monetized annual health impacts due to Chronic $\mathrm{NO}_{2}$ exposure from various $12 \mathrm{~km}$ (fine) and $36 \mathrm{~km}$ (coarse) input resolution scenarios and the differences between the respective scenario and the all fine $12 \mathrm{~km}$ model run 

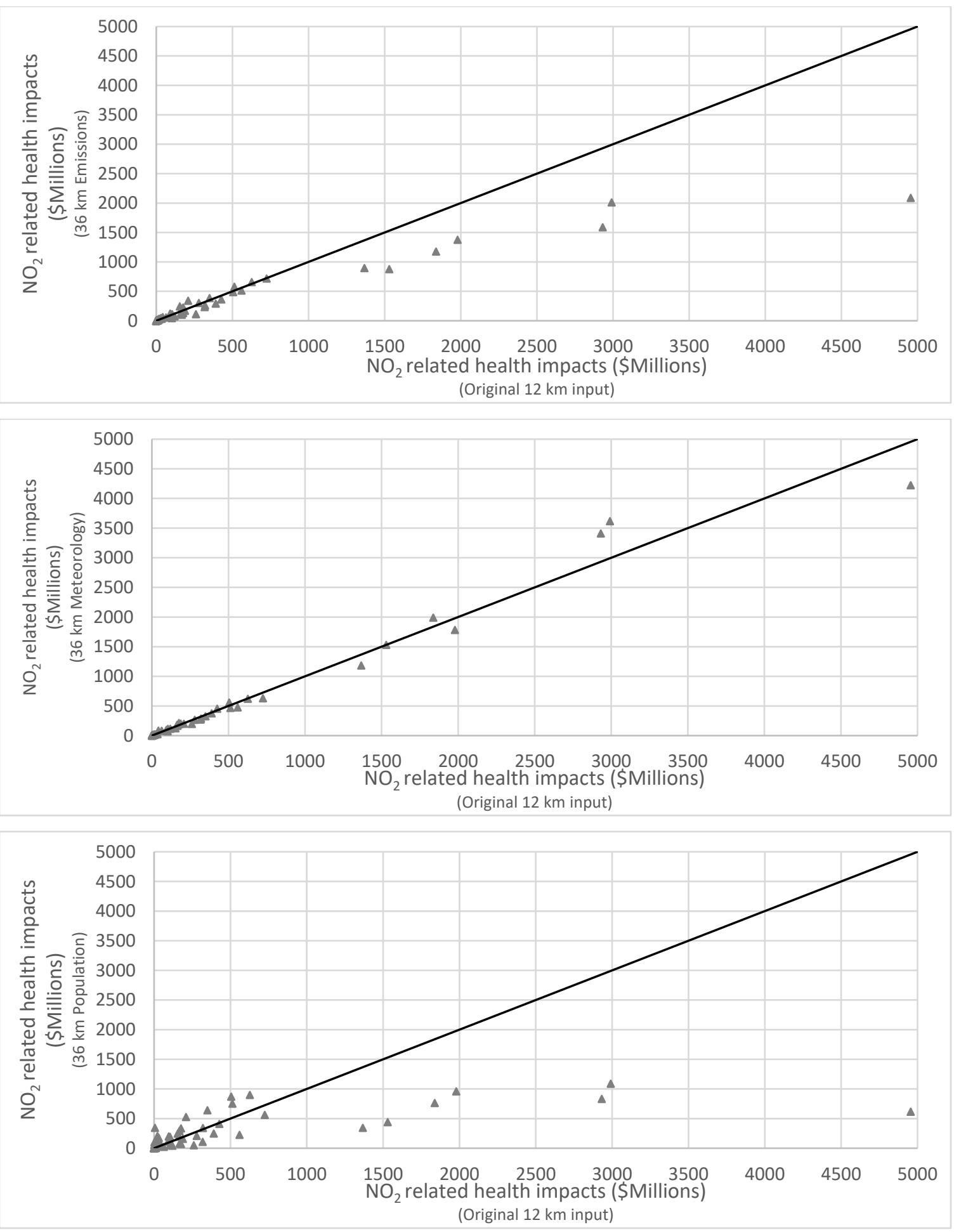

Figure 5-39. Scatter plots comparing the monetized health impacts due to chronic $\mathrm{NO}_{2}$ exposure results for combinations of fine $12 \mathrm{~km}$ resolution with coarse $36 \mathrm{~km}$ inputs: emissions (top), meteorology (middle), and population (bottom). 
The chronic health impacts, parameterized by monetized mortality, due to chronic exposure to $\mathrm{NO}_{2}$ for the 4 and $12 \mathrm{~km}$ resolution scenarios are displayed in Figure 5-40 for each input parameter, and in Figure 5-41 as scatter plots with the coarse $12 \mathrm{~km}$ input results on the $y$-axis and the original fine $4 \mathrm{~km}$ input results on the $\mathrm{x}$-axis.

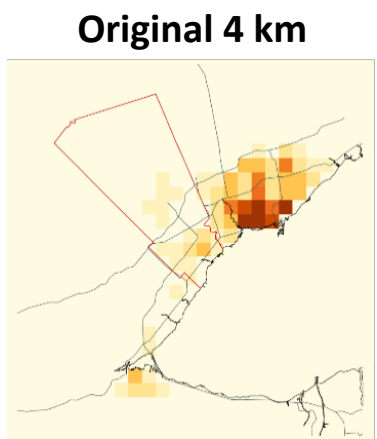

Maximum: \$1.36B
12 km Emissions

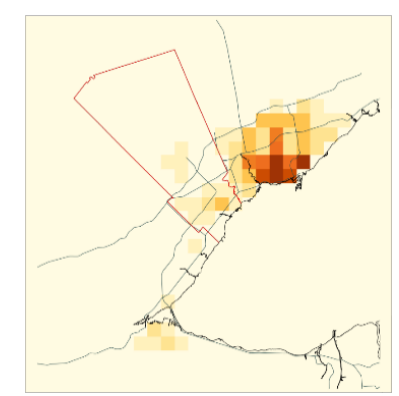

Maximum: \$1.22B
12 km Meteorology

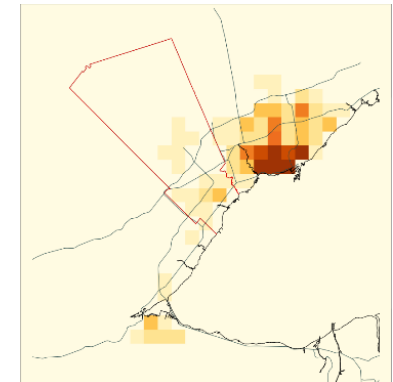

Maximum: \$1.51B
12 km Population

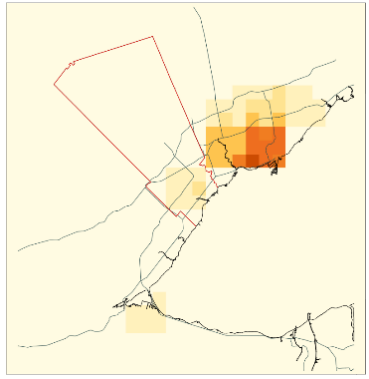

Maximum: \$0.76B

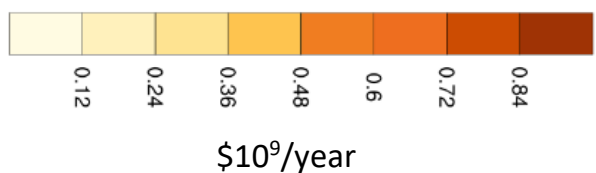

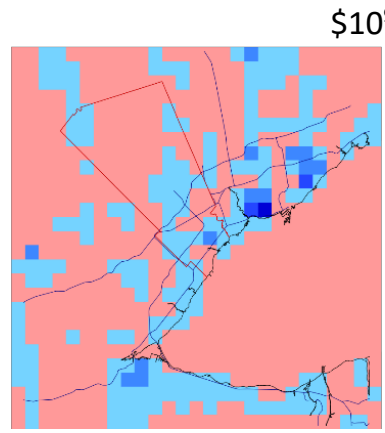

Max. Difference: \$0.0278B Minimum Difference: $-\$ 0.143$

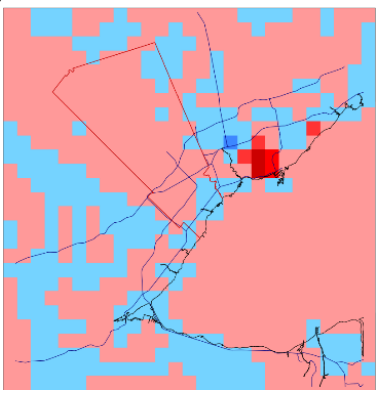

Maximum Difference: $\$ 0.145$ Minimum Difference: $-\$ 0.076$

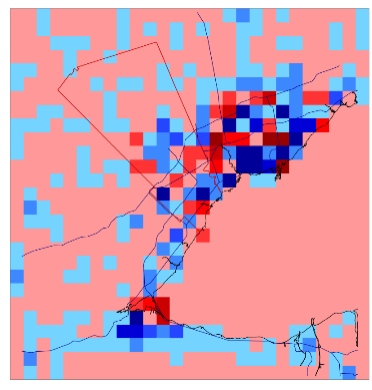

Maximum Difference: $\$ 0.439$ Minimum Difference: $-\$ 0.597$

Figure 5-40. Monetized annual health impacts due to Chronic $\mathrm{NO}_{2}$ exposure from various $4 \mathrm{~km}$ (fine) and $12 \mathrm{~km}$ (coarse) input resolution scenarios and the differences between the respective scenario and the all fine $4 \mathrm{~km}$ model run. 


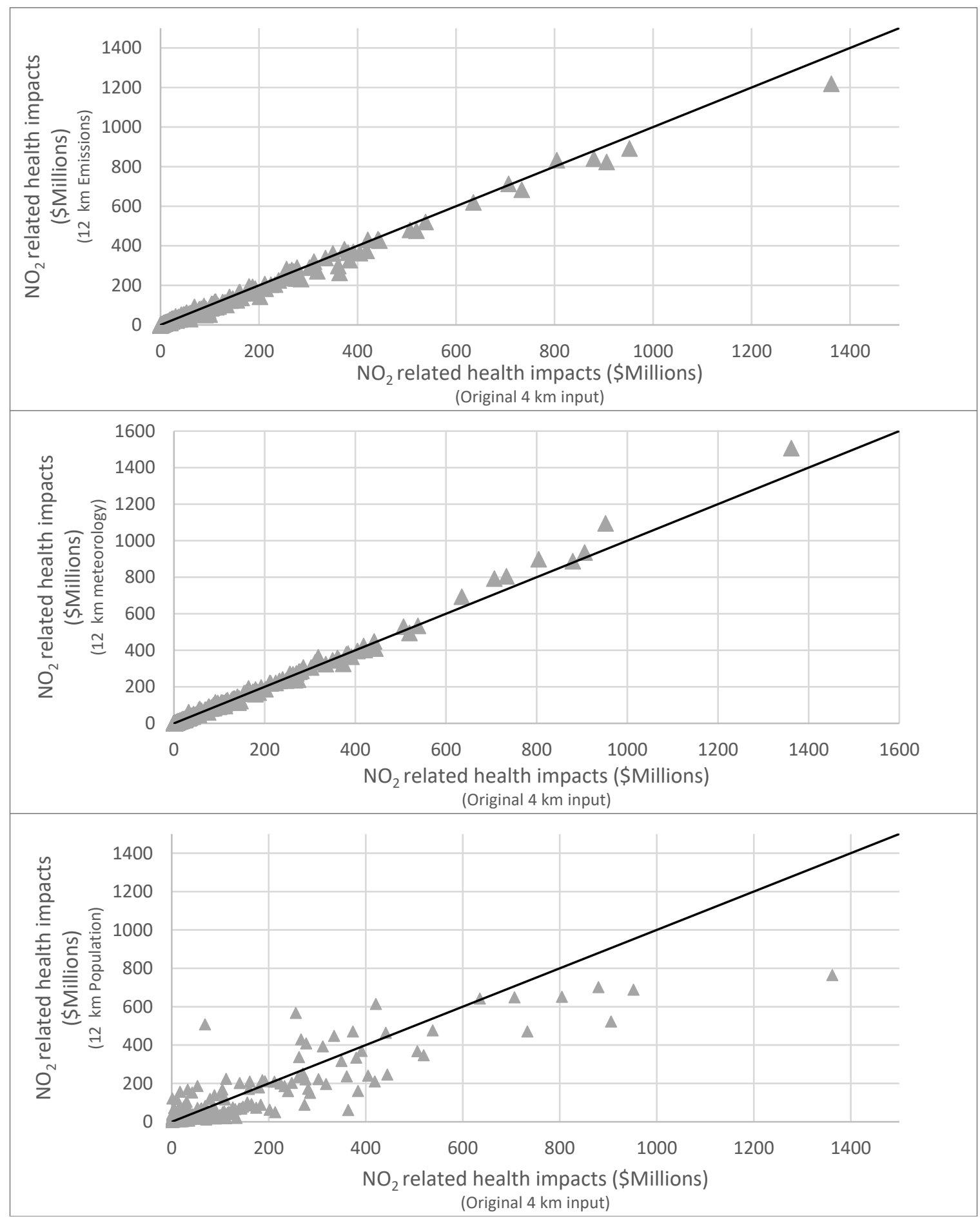

Figure 5-41. Scatter plots comparing the monetized health impacts due to chronic $\mathrm{NO}_{2}$ exposure results for combinations of fine $4 \mathrm{~km}$ resolution with coarse $12 \mathrm{~km}$ inputs: emissions (top), meteorology (middle), and population (bottom). 
The chronic health impacts, parameterized by monetized mortality, due to chronic exposure to $\mathrm{NO}_{2}$ for the 1 and $4 \mathrm{~km}$ resolution scenarios are displayed in Figure 5-40 for each input parameter, and in Figure 5-41 as scatter plots with the coarse $4 \mathrm{~km}$ input results on the $\mathrm{y}$-axis and the original fine $1 \mathrm{~km}$ input results on the $\mathrm{x}$-axis.

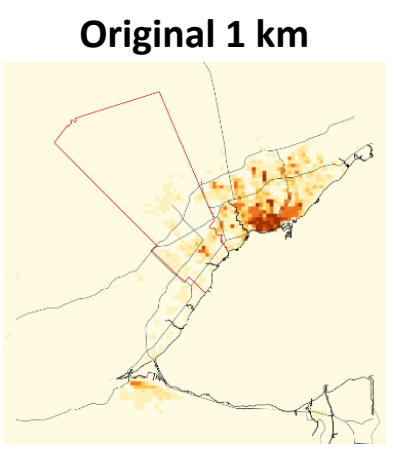

Maximum: $\$ 220.1 \mathrm{M}$

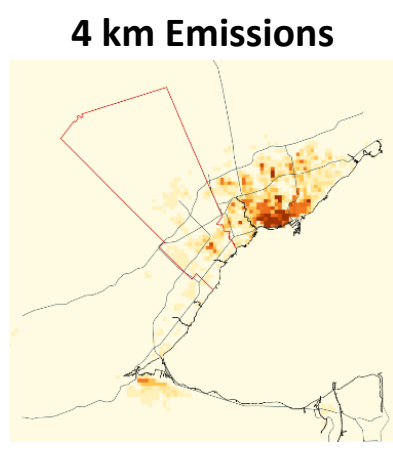

Maximum: \$196.9M

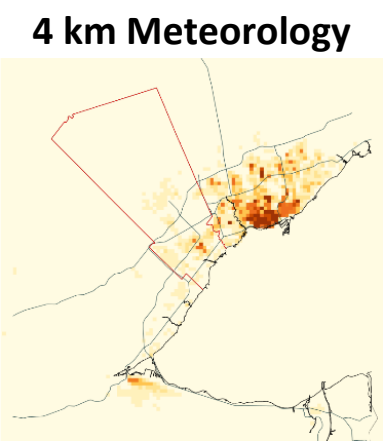

Maximum: $\$ 220.9 \mathrm{M}$

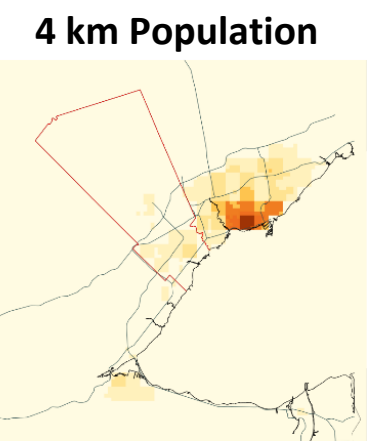

Maximum: $\$ 79.0 \mathrm{M}$

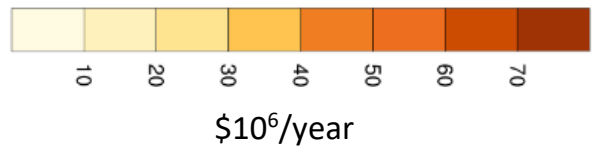

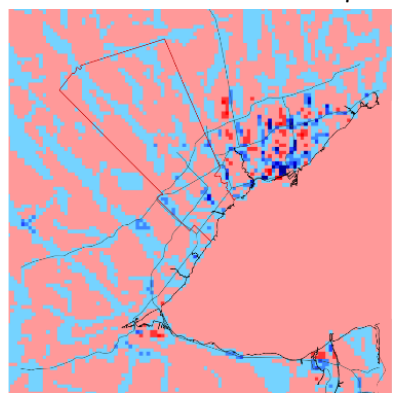

Maximum Difference: $\$ 2.88 \mathrm{M}$

Min. Difference: -\$23.19M

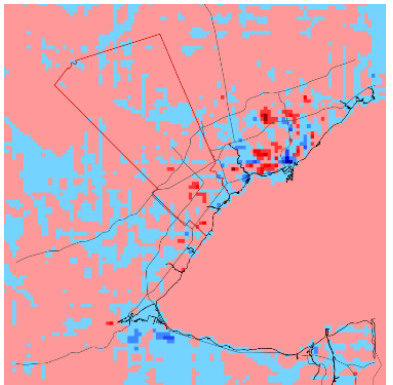

Maximum Difference: $\$ 7.86 \mathrm{M}$

Minimum Difference: $-\$ 6.59 \mathrm{M}$

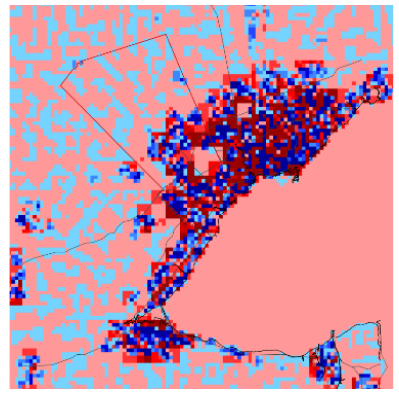

Maximum Difference: $\$ 45.65 \mathrm{M}$ Min. Difference: $-\$ 165.03 M$

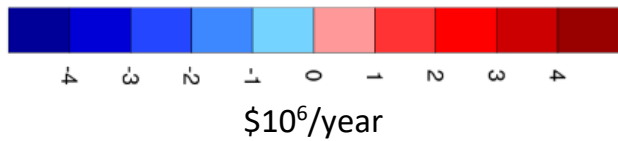

Figure 5-42. Monetized annual health impacts due to Chronic $\mathrm{NO}_{2}$ exposure from various $4 \mathrm{~km}$ (fine) and $12 \mathrm{~km}$ (coarse) input resolution scenarios and the differences between the respective scenario and the all fine $4 \mathrm{~km}$ model run. 


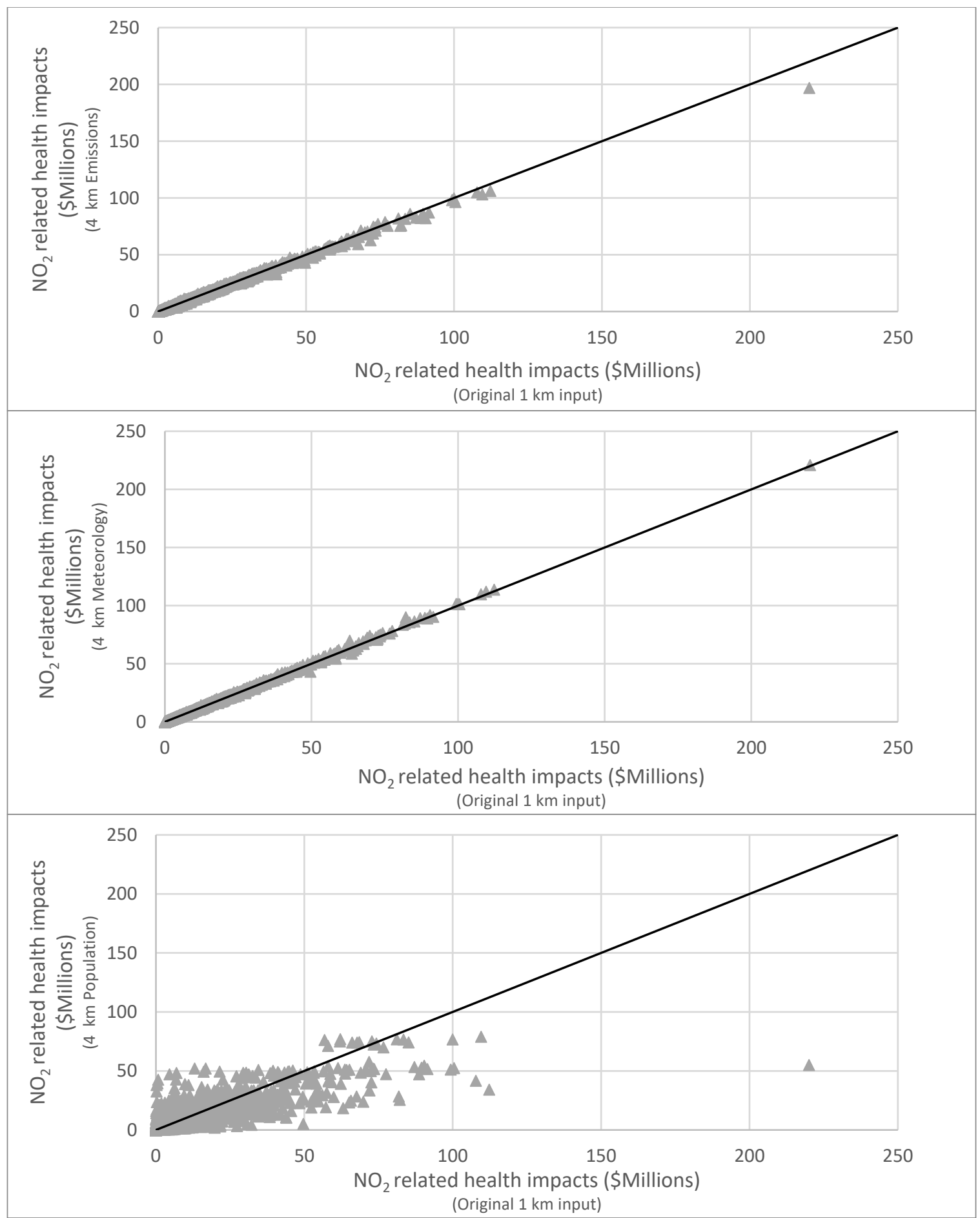

Figure 5-43. scatter plots comparing the monetized health impacts due to chronic $\mathrm{NO}_{2}$ exposure results for combinations of fine $1 \mathrm{~km}$ resolution with coarse $4 \mathrm{~km}$ inputs: emissions (top), meteorology (middle), and population (bottom). 
The chronic health impacts, parameterized by monetized mortality, due to chronic exposure to $\mathrm{PM}_{2.5}$ for the 12 and $36 \mathrm{~km}$ resolution scenarios are displayed in Figure 5-44 for each input parameter, and in Figure 5-45 as scatter plots with the coarse $36 \mathrm{~km}$ input results on the $y$-axis and the original fine $12 \mathrm{~km}$ input results on the $\mathrm{x}$-axis.

\section{Original 12 km}

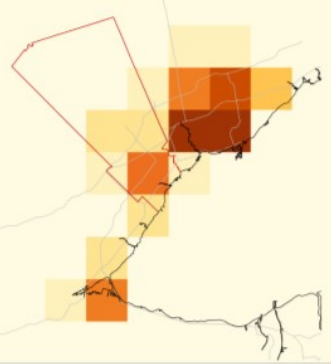

Maximum: \$14.8B

\section{6 km Emissions}

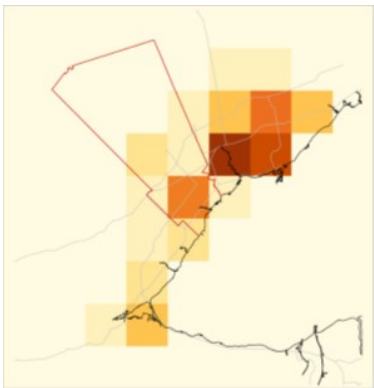

Maximum: \$10.5B
36 km Meteorology

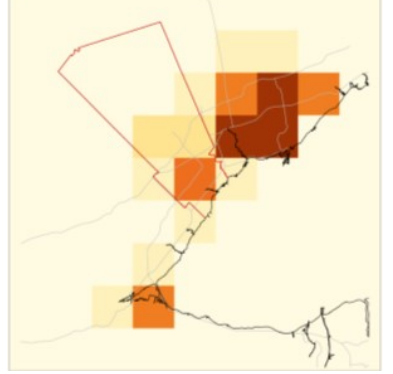

Maximum: \$13.6B
36 km Population

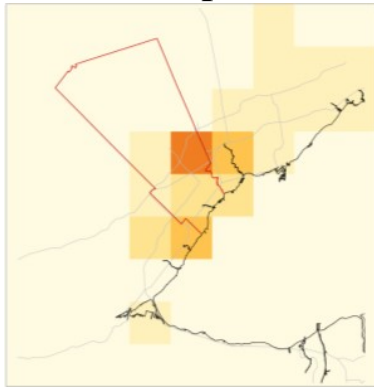

Maximum: \$6.1B

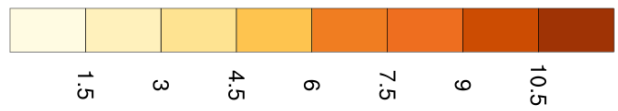

$\$ 10^{9} /$ year

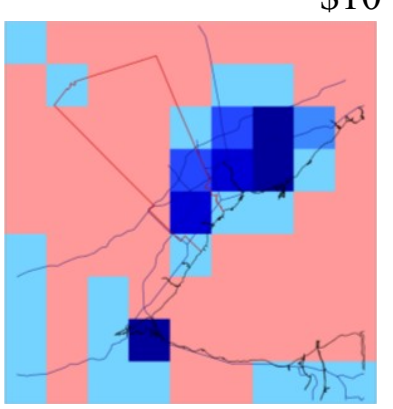

Maximum Difference $\$ 0.45 B$ Minimum Difference: $\$ 4.35 B$

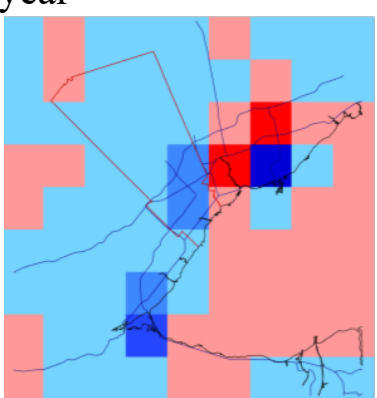

Maximum Difference: $\$ 1.20 \mathrm{~B}$ Minimum Difference: $-\$ 1.96 \mathrm{~B}$

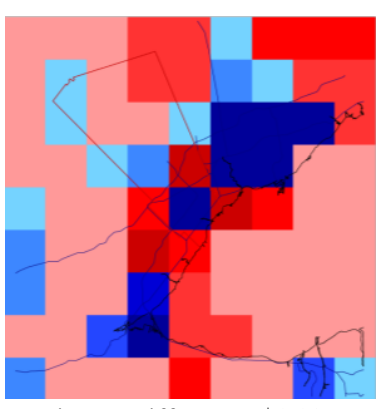

Maximum Difference: \$1.98B Minimum Difference: $-\$ 13.00 \mathrm{~B}$

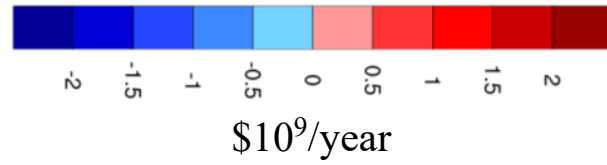

Figure 5-44. Monetized annual health impacts due to Chronic $P M_{2.5}$ exposure from various $12 \mathrm{~km}$ (fine) and $36 \mathrm{~km}$ (coarse) input resolution scenarios and the differences between the respective scenario and the all fine $12 \mathrm{~km}$ model run. 

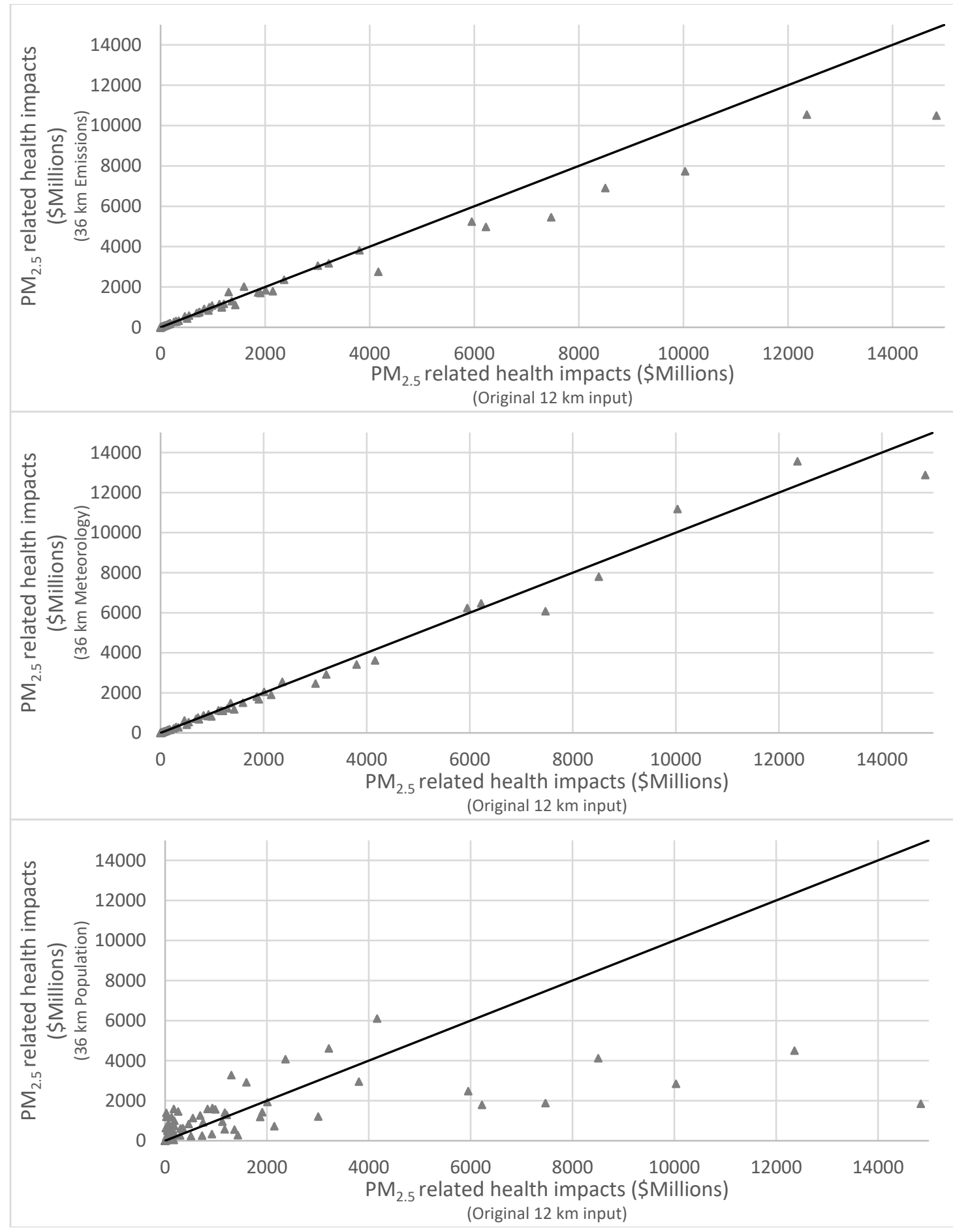

Figure 5-45 scatter plots comparing the monetized health impacts due to chronic $\mathrm{PM}_{2.5}$ exposure results for combinations of fine $12 \mathrm{~km}$ resolution with coarse $36 \mathrm{~km}$ inputs: emissions (top), meteorology (middle), and population (bottom). 
The chronic health impacts, parameterized by monetized mortality, due to chronic exposure to $\mathrm{PM}_{2.5}$ for the 4 and $12 \mathrm{~km}$ resolution scenarios are displayed in Figure 5-46 for each input parameter, and in Figure 5-47 as scatter plots with the coarse $12 \mathrm{~km}$ input results on the $y$-axis and the original fine $4 \mathrm{~km}$ input results on the $\mathrm{x}$-axis.

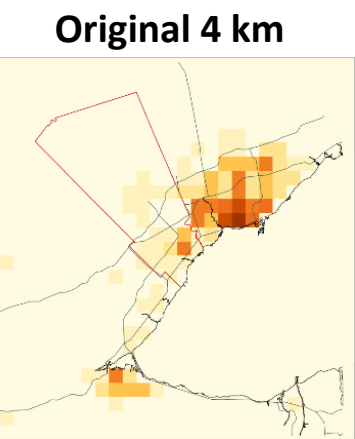

Maximum: $\$ 3.45 B$
12 km Emissions

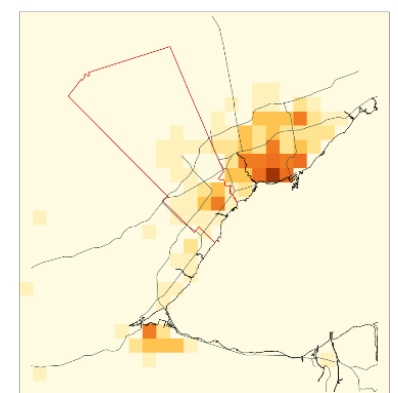

Maximum: $\$ 3.18 \mathrm{~B}$
12 km Meteorology

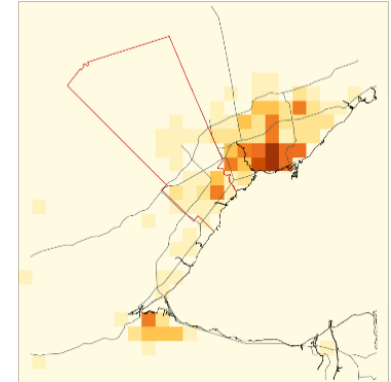

Maximum: $\$ 3.81 \mathrm{~B}$

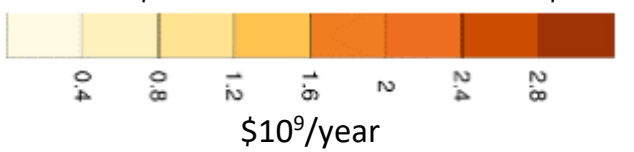

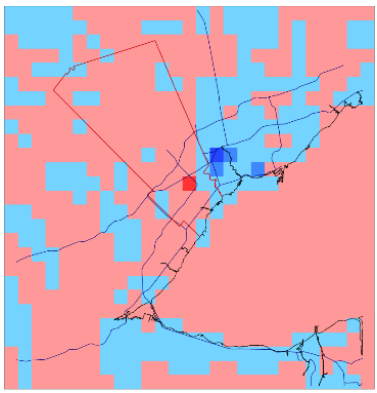

Maximum Difference: $\$ 0.266 \mathrm{~B}$ Minimum Difference: $-\$ 0.472$

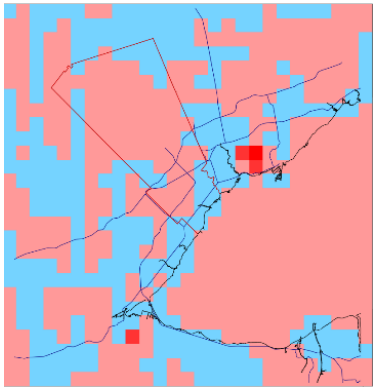

Maximum Difference: $\$ 0.512 B$ Minimum Difference: $-\$ 0.190 B$

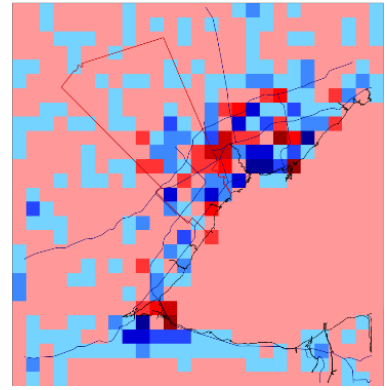

Maximum Difference: $\$ 1.34 \mathrm{~B}$ Minimum Difference: $-\$ 1.51 \mathrm{~B}$

\section{2 km Population}

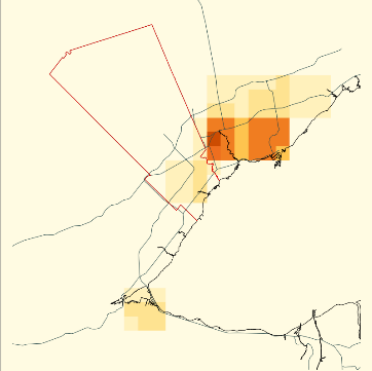

Maximum: $\$ 2.43$

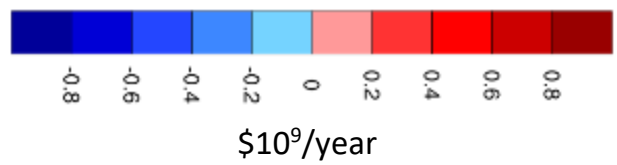

Figure 5-46. Monetized annual health impacts due to Chronic $\mathrm{PM}_{2.5}$ exposure from various $4 \mathrm{~km}$ (fine) and $12 \mathrm{~km}$ (coarse) input resolution scenarios and the differences between the respective scenario and the all fine $4 \mathrm{~km}$ model run. 


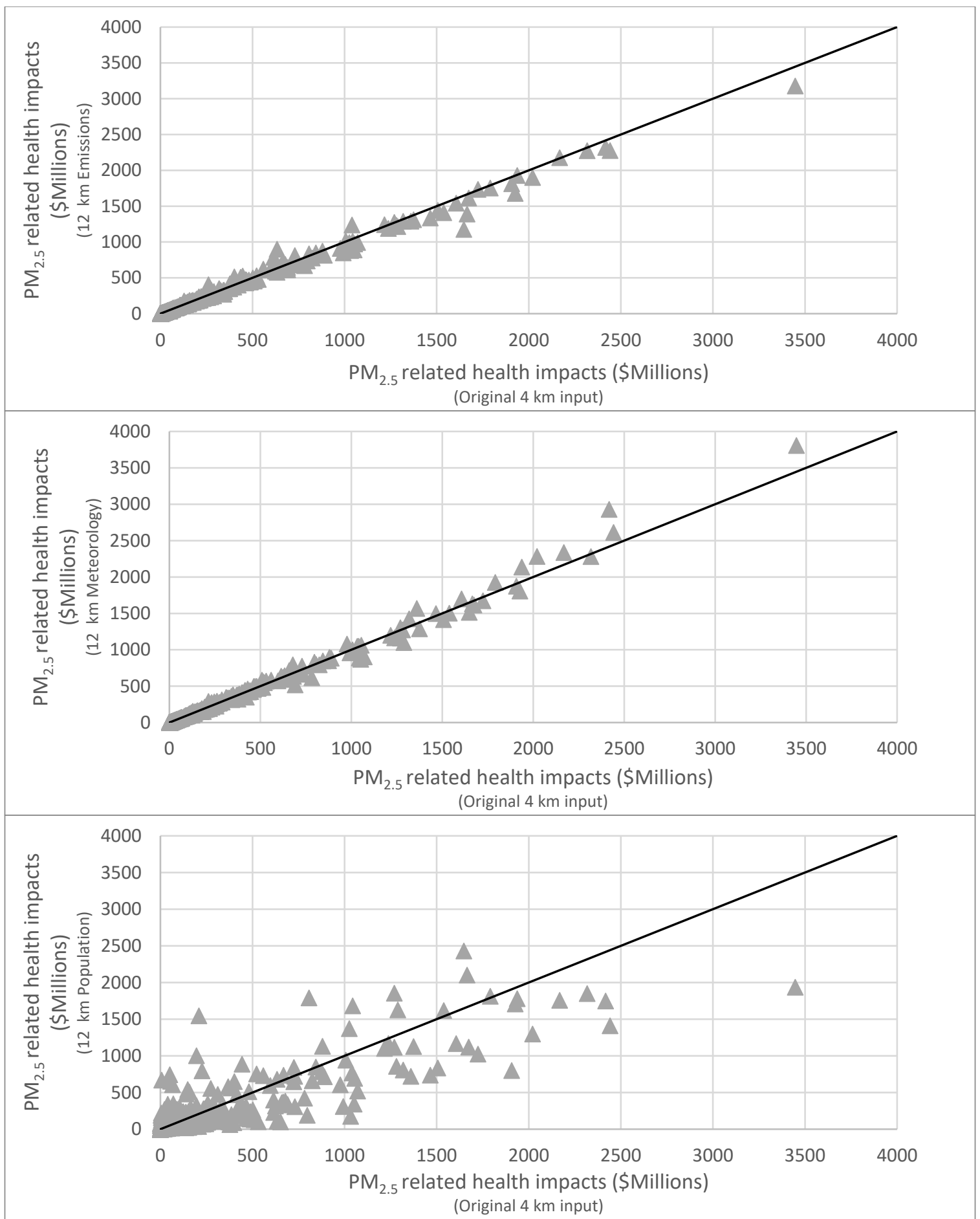

Figure 5-47. scatter plots comparing the monetized health impacts due to chronic $\mathrm{PM}_{2.5}$ exposure results for combinations of fine $4 \mathrm{~km}$ resolution with coarse $12 \mathrm{~km}$ inputs: emissions (top), meteorology (middle), and population (bottom). 
The chronic health impacts, parameterized by monetized mortality, due to chronic exposure to $\mathrm{PM}_{2.5}$ for the 1 and $4 \mathrm{~km}$ resolution scenarios are displayed in Figure 5-48 for each input parameter, and in Figure 5-49 as scatter plots with the coarse $12 \mathrm{~km}$ input results on the $y$-axis and the original fine $4 \mathrm{~km}$ input results on the $\mathrm{x}$-axis.

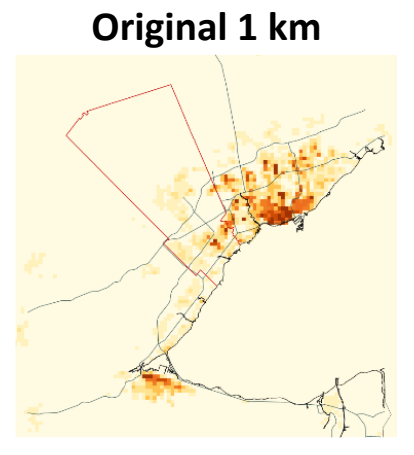

Maximum: \$596.4M

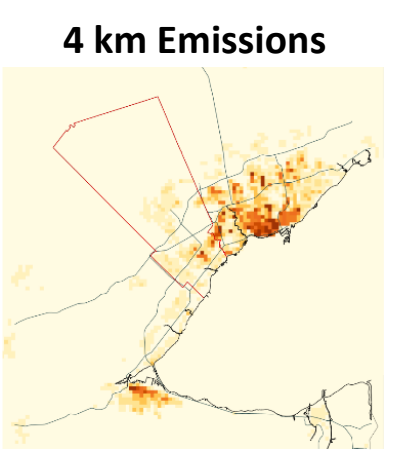

Maximum: \$530.1M

\section{4 km Meteorology}

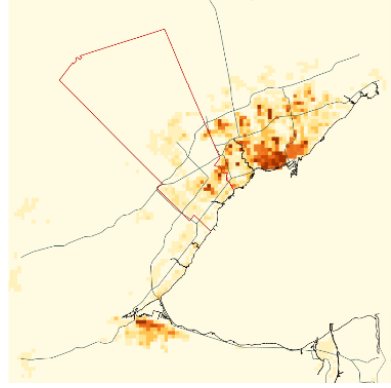

Maximum: $\$ 608.6 \mathrm{M}$

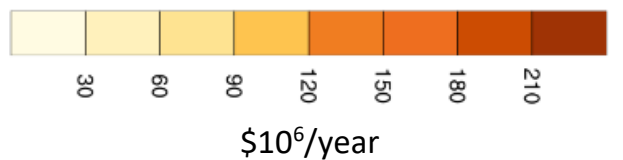

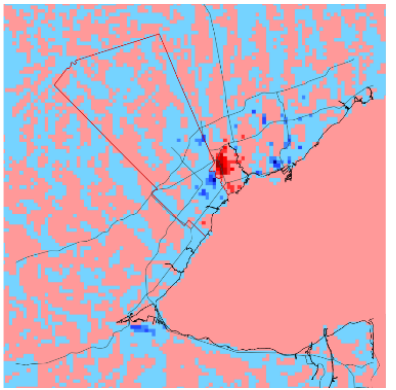

Maximum Difference: $\$ 68.39 \mathrm{M}$ Min. Difference: $-\$ 66.29 \mathrm{M}$

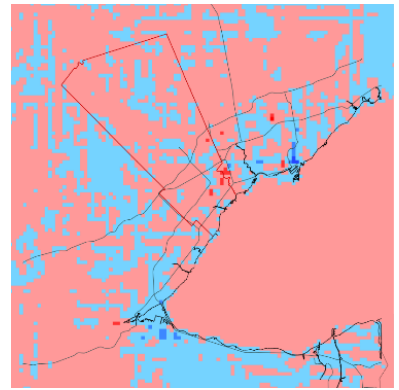

Maximum Difference: $\$ 16.52 \mathrm{M}$

Min. Difference: $-\$ 15.26 \mathrm{M}$

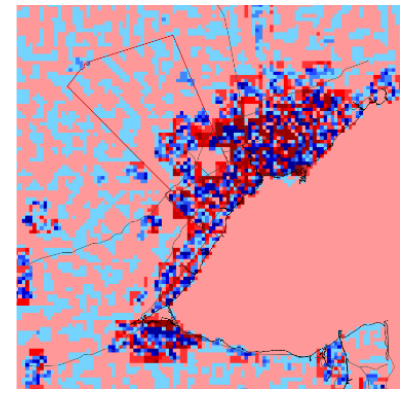

Max. Difference: $\$ 124.15 \mathrm{M}$ Min. Difference: $-\$ 447.24 \mathrm{M}$
4 km Population

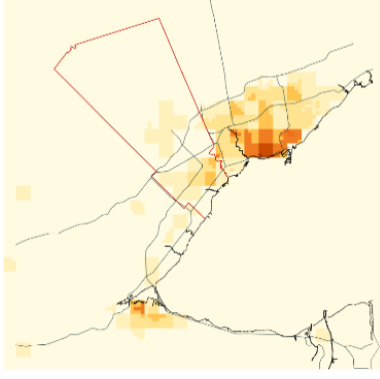

Maximum: \$219.0M

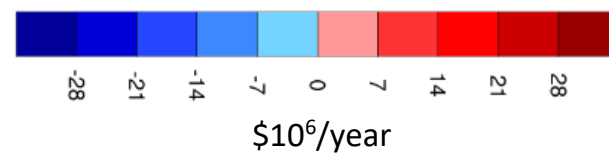

Difference with

Original:

$\$ 10 \%$ year

Figure 5-48. Monetized annual health impacts due to Chronic $\mathrm{PM}_{2.5}$ exposure from various $1 \mathrm{~km}$ (fine) and $4 \mathrm{~km}$ (coarse) input resolution scenarios and the differences between the respective scenario and the all fine $1 \mathrm{~km}$ model run. 


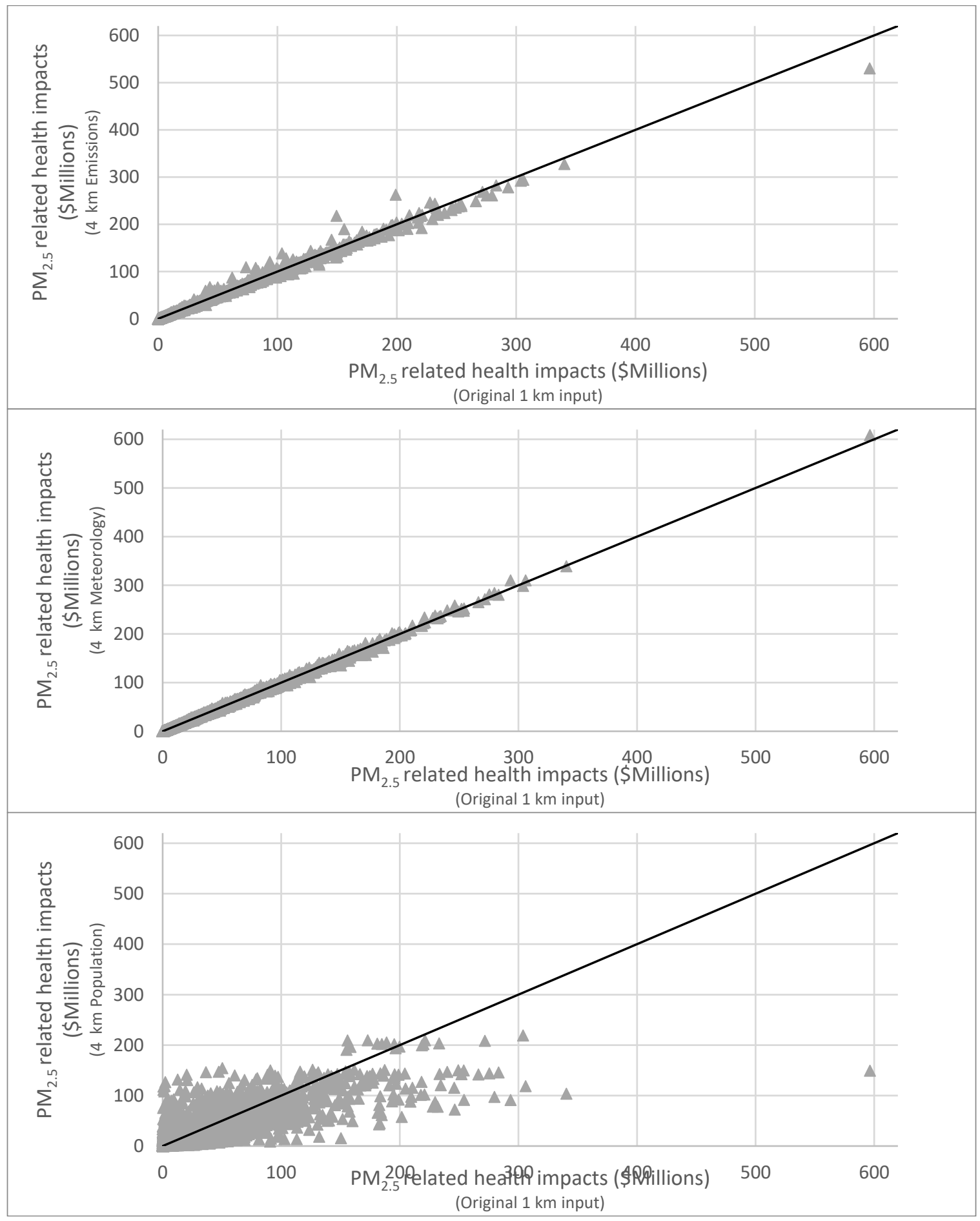

Figure 5-49. Scatter plots comparing the monetized health impacts due to chronic $\mathrm{PM}_{2.5}$ exposure results for combinations of fine $1 \mathrm{~km}$ resolution with coarse $4 \mathrm{~km}$ inputs: emissions (top), meteorology (middle), and population (bottom). 


\subsection{Conclusion}

The research presented within this thesis contributes to the air quality field as it is novel regarding analyzing the impacts of grid resolution on health impacts estimated with the usage of adjoint sensitivity analysis. It is also original in analyzing the impacts of specific CTM input processes on health estimates, as opposed to being limited to analyzing the resolution of all inputs collectively. This research has effectively examined and added insight to the four research questions presented in Chapter 1 (and summarized below):

1. What is the extent of impact of horizontal grid resolution on domain-wide predicted health impacts related to pollution exposure?

2. How does fine resolution modelling influence spatial and temporal patterns related to health benefits seen by emission reductions?

3. How does the resolution of specific model inputs (e.g. meteorology, emissions, population) impact health estimates?

4. Which CTM input provides a greater benefit to be of fine resolutions?

The research presented in Chapter 4 presents responses to the above questions 1 and 2 . It successfully quantifies the extent of impact that horizontal grid resolution has on domain-wide predicted health impacts related to pollution exposure. The results indicate that over a large domain, the accumulated health impact does not drastically change between coarse and fine resolution. If the purpose of a study was to quantify the total health impacts over a domain, then choosing a resolution as fine as $1 \mathrm{~km}$ is not essential. 
Results in this study also demonstrate the strong spatial variability at fine resolution modelling, which is lost when switched to coarse. A benefit of high spatial representation is that it allows emission reduction efforts to be focused on locations in which the highest impacts will be generated. In urban areas, there is drastically higher spatial variance seen at $1 \mathrm{~km}$ resolution than in rural areas. This brings insight that fine resolution modelling has a greater benefit in urban areas where there are drastic spatial gradients of emissions, populations, and concentrations, than compared to rural areas. One may justify modelling only urban areas at fine resolution, while modelling more urban areas at coarse resolutions.

Chapter 5 further investigated the influences of grid resolution by examining the impact that resolution has on each individual input parameter and in doing so touches on responses to questions 1,3 , and 4 . For concentration predictions, it was found that the most beneficial input (out of emissions or meteorology) to have fine resolution data for was dependent on the species. Ozone was more sensitive to the resolution of meteorology, whereas $\mathrm{NO}_{2}$ and $\mathrm{PM}_{2.5}$, were more sensitive to the resolution of emissions. When estimating health impacts, the most beneficial input to have fine resolution data for, regardless of the species of analysis, was population, followed by that of concentration.

The results presented within both chapters 4 and 5 verify that the impact of resolution, whether with respect to individual inputs or all inputs collectively, depends on the species of analysis. Generally, $\mathrm{PM}_{2.5}$ and $\mathrm{NO}_{2}$ were more sensitive to grid resolution impacts than $\mathrm{O}_{3}$ was. A correlation between atmospheric lifetime and sensitivity to resolution can be drawn. Short- 
lived species are less spatially homogenous due to short dispersal ranges resulting in greater spatial gradients that are not well represented at coarse resolutions.

There are various limitations within this research and areas for consideration of improvement in future work. The relatively short simulation periods in both studies may cause them to not be temporally representative. This is especially prominent in chapter 5 , in which the period was only 2-days due to the high number of required iterations. Meteorology is a dynamic parameter and it must be acknowledged that to capture a full representation of meteorology an extended period would be required. The MB results presented in chapter 4 were combined for $\mathrm{O}_{3}$ and $\mathrm{NO}_{2}$, two species proven to behave differently to resolution impacts. Estimating the impacts of resolution on these two species individually would prove beneficial. Future work could expand the analysis to other regions to validate whether the conclusions are spatially dependent. The location of analysis was near a large body of water, it is anticipated that an inland location may find different results. Additional limitations are presented within chapters 4 and 5 .

This research hopes to shed light on the importance of model resolution on concentration and health impact predictions. Fine resolution modelling is not always justifiable and one must consider the purpose of the analysis prior to assuming that fine-grid resolutions will improve overall results. 


\section{Bibliography}

Anenberg, S. C., Horowitz, L.W., Tong, D.Q., and J. J. West. 2010. “An estimate of the global burden of anthropogenic ozone and fine particulate matter on premature human mortality using atmospheric modeling." Environmental Health Perspectives 118(9): $1189-1195$.

Arunachalam, S., Holland, A., Do, B., and M. Abraczinskas. 2006. "A quantitative assessment of the influence of grid resolution on predictions of future-year air quality in North Carolina, USA." Atmospheric Environment 40: 5010-5025.

Arunachalam, S., Wang, B., Davis, N., Baek, B.H., and J.I. Levy. 2011. "Effect of chemistrytransport model scale and resolution on population exposure to $\mathrm{PM}_{2.5}$ from aircraft emissions during landing and takeoff." Atmospheric Environment 45: 3294-3300.

Baklanov, A., Rasmussen, A., Fay, B., Berge, E., and S. Finardi. 2002. “Potential and shortcomings of numerical weather prediction models in providing meteorological data for urban air pollution forecasting." Water, Air, and Soil Pollution: Focus 2: 43-60.

Bell, M. L., McDermott, A., Zeger, S. L., Samet, J. M., and Dominici, F. 2004. “Ozone and shortterm mortality in 95 US urban communities, 1987-2000." Journal of the American Medical Association 292(19): 2372-2378.

Boersma, K.F., Eskes, H.J., Veefkind, J.P., Brinksma, E.J., van der A., R.J., Sneep, M., van den Oord, G.H.J., Levelt, P.F., Stammes, P., Gleason, J.F., and E.J. Bucsela. 2007. “Near-real time retrieval of tropospheric NO2 from OMI," Atmospheric Chemistry and Physics 7:2103-2118. 
Burnett, R.T., Stieb, D., Brook, J.R., Cakmak, S., Dales, R., Raizenne, M., Vincent, R., and T. Dann. 2004. "Associations between short-term changes in nitrogen dioxide and mortality in Canadian cities." Archives of Environmental Health 59(5): 228-236.

Burnett, R., Chen, H., Szyszkowicz, M., Fann, N., Hubbell, B., Pope-III, C.A., Apte, J.S., Brauer, M., Cohen, A., Weichenthal, S., et al. 2018. "Global estimates of mortality associated with long-term exposure to outdoor fine particulate matter." Proceedings of the National Academy of Sciences of the United States of America 115(38) 9592-9597.

Byun, D., abd J.K.S. Ching. 1999. "Science algorithms of the EPA models-3 community multiscale air quality (CMAQ) modelling system." U.S. EPA. EPA/600/R-99/030.

Byun, D., and K.L. Schere. 2006. "Review of the governing equations, computational algorithms, and other components of the Models-3 Community Multiscale Air Quality (CMAQ) modeling system." Applied Mechanics Review 59(2): 51-77.

Canmak, S., Hebbern, C., Pinault, L., Lavigne, E., Vanos, J., Crouse, D.L. and M. Tjepkema. 2018. "Associations between long-term PM2.5 and ozone exposure and mortality in the Canadian Census Health and Environment Cohort (CANCHEC), by spatial synoptic classification zone." Environment International 111: 200-211.

Chen, B., and H. Kan. 2008. "Air pollution and population health: a global challenge." Environmental Health and Preventive Medicine 13: 94-101.

Chestnut, L.G. and P. De Civita. 2009. “Economic Valuation of Mortality Risk Reduction: Review and Recommendations for Policy and Regulatory Analysis." Policy Research Initiative, Government of Canada. 
Cohan, D.S., Hu, Y., and A.G. Russel. 2006. “Dependence of ozone sensitivity analysis on grid resolution." Atmospheric Environment 40: 126-135.

Colle, B.A., Olson, J.B., and J.S. Tongue. 2003. "Multiseason verification of the MM5. Part I: comparison with the Eta model over the central and eastern United States and impact of MM5 resolution". Weather and Forecasting 18(3): 431-457.

Community Modeling and Analysis System (CMAS). 2012. “Operational guidance for the Community Multiscale Air Quality (CMAQ) modeling system.” Retrieved January 13, 2018 from:

https://www.airqualitymodeling.org/index.php/CMAQ_version_5.0_(February_2010_re lease)_OGD\#GRIDDESC:_Horizontal_domain_definition

Crouse, D.L., Peters, P.A., Hystad, P. Brook, J.R., van Donkelaar, A., Marin, R.V., Villeneuve, P.J., Jerrett, M., Goldberg, M.S., Arden Pope III, C. Brauer, M., Brook, R.D., Robichaud, A., Menard, R., and R.T. Burnett. 2015. “Ambient $\mathrm{PM}_{2.5}, \mathrm{O}_{3}, \mathrm{NO}_{2}$ exposures and associations with mortality over 16 years of follow-up in the Canadian Census Health and Environment Cohort (CanCHEC)." Environmental Health Perspectives 123: 1180-1186.

De Meij, A., Wagner, S., Cuvelier, C., Dentener, F., Gobron, N., Thunis, P., and M. Schaap. 2007. "Model evaluation and scale issues in chemical and optical aerosols properties over the greater Milan area (Italy), for June 2001." Atmospheric Research 85(2): 243-267.

Dockery, D.W., Pope, C.A., Xu, X., Spengler, J.D., et al. 1993. "An association between air pollution and mortality in six U.S. cities." The New England Journal of Medicine 329: 1753-1759. 
Eftim, S.E., Samet, J.M., Janes, H., McDermott, A., and F. Dominici. 2008. "Fine particulate matter and mortality: a comparison of the six cities and American Cancer Society cohorts with a medicare cohort." Epidemiology 19(2): 209-2016.

Environment and Climate Change Canada (ECCC). 2013a. “Canadian Ambient Air Quality Standards." Retrieved January 13, 2018 from: http://www.ec.gc.ca/default.asp?lang=En\&n=56D4043B-1\&news=A4B2C28A-2DFB4BF4-8777-ADF29B4360BD.

Environment and Climate Change Canada (ECCC). 2013b. Annex 3: Specific Objectives Concerning Ground-level Ozone Precursors. Retrieved from: https://www.ec.gc.ca/air/default.asp?lang=En\&n=9992B080-1.

Esler, J.G., Roelods, G.J., Köhler, M.O., and F.M. O'Connor. 2004. “A quantitative analysis of grid-related systematic errors in oxidising capacity and ozone production rates in chemistry transport models." Atmospheric Chemistry and Physics 4(7): 1781-1795.

Faustini, A., Rapp, R., and F. Foratiere. 2014. “Nitrogen dioxide and mortality: review and metaanalysis of long-term studies." European Respiratory Journal 44: 744-763.

Fenger, J. 2003. "Urban-scale air pollution." Handbook of Atmospheric Science: Principles and Applications. Blackwell Publishing. 399-438.

Fountoukis, C., Koraj, D.H., Denier van der Gon, H.A.C., Charalampidis, P.E., Pilinis, C., and S.N. Pandis. 2013. "Impact of grid resolution on the predicted fine PM by a regional 3-D chemical transport model." Atmospheric Environment 68: 24-32.

Gallus, W. 1999. “Eta Simulations of Three Extreme Precipitation Events: Sensitivity to Resolution and Convective Parameterization." Weather and Forecasting 14(3): 405-426. 
Gao, X., Xu, Y., Zhao, Z., Pal, J. and F. Giorgi. 2006. “On the role of resolution and topography in the simulation of East Asia precipitation." Theoretical and Applied Climatology 86(1-4): $173-185$.

Gillani, N.V., and J.E. Pleim. 1996. "Sub-grid-scale features of anthropogenic emissions of NOx and VOC in the context of regional eulerian models." Atmospheric Environment 30: 2043-2059.

Grabowski, W.W., Wu, X., Moncrieff, M.W., and W.D. Hall. 1998. "Cloud-Resolving modeling of cloud systems during Phase II of GATE, Part IIL Effects of resolution and the third spatial dimension." Journal of the Atmospheric Sciences 55: 3264-3282.

Hakami, A., Henze, D.K., Seinfeld, J.H., Singh, K., Sandu, A., Kim, S., and Q. Li. 2007. “The adjoint of CMAQ. Environmental Science \& Technology 41(22): 7807-7817

Hanna, S.R., Changa, J.C., and M.E. Fernauna. 1998. "Monte carlo estimates of uncertainties in predictions by a photochemical grid model (UAM-IV) due to uncertainties in input variables." Atmospheric Environment 32(21): 3619-3628.

Hanna, S.R., Lu, Z., Frey, H.C., Wheeler, N., Vukovich, J., Arunachalam, S., Fernau, M., and D.A. Hansen. 2001. "Uncertainties in predicted ozone concentrations due to input uncertainties for the UAM-V photochemical grid model applied to the July 1995 OTAG domain." Atmospheric Environment 35(5): 891-903.

Henze, D.K., Hakami, A., and J.H. Seinfeld. 2007. "Development of the adjoint of GEOS-Chem." Atmospheric Chemistry and Physics 7: 2413-2433.

Hodnebrog, Ø., Stordal, F., and T.K. Berntsen. 2011. "Does the resolution of megacity emissions impact large scale ozone?" Atmospheric Environment 45(38): 6852-6862. 
Im, U. and M. Kanakidou. 2012. "Impacts of East Mediterranean megacity emissions on air quality." Atmospheric Chemistry and Physics 12(14): 6335-6355.

Jacob, D.J. 2007. "Atmospheric Models of atmospheric transport and chemistry." Lectures on Inverse Modelling, Harvard University. Retrieved April 24, 2018 from: http://acmg.seas.harvard.edu/education/jacob_lectures_ctms_chap1.pdf Jacob, D.J. 1999. "Introduction to Atmospheric Chemistry." Princeton University Press. Jang, J.C., Jeffries, H.E., Byun, D., and J.E. Pleim. 1995. "Sensitivity of ozone to model grid resolution - I. Application of high-resolution regional acid deposition model." Atmospheric Environment 29(21): 3085-3100.

Jang, J.C., Jeffries, H.E., and S. Tonnesen. 1995. "Sensitivity of ozone to model grid resolution II. Detailed process analysis for ozone chemistry." Atmospheric Environment 29(21): 3101-3114.

Jerrett, M., Burnett, R., Pope, C., Ito, K., Thurston, G., Krewski, D., Shi, Y., Calle, E. and M. Thun. 2009. “Long-Term Ozone Exposure and Mortality." New England Journal of Medicine 360(11): 1085-1095.

Jimenez, P., Jorba, O., Parra, R., and J.M. Baldasano. 2006. “Evaluation of MM5-EMICAT2000CMAQ performance and sensitivity in complex terrain: high-resolution application to the northeastern Iberian Peninsula." Atmospheric Environment 40 (26): 5056-5072.

Judek S, Stieb D, and B. Jovic. 2006. Air Quality Benefits Assessment Tool (AQBAT) release 1.0. Health Canada. 
Katsouyanni K., Samet, J.M., Anderson, H.R., Atkinson, R., Le Terte A., et al. 2009. “Air pollution and health: a European and North American Approach (APHENA)." Research Report, Health Effects Institute 142: 5-90.

Krewski, D., Jerrett, M., Burnett, R. T., Ma, R., Hughes, E., Shi, Y., et al. 2009. “Extended followup and spatial analysis of the American Cancer Society study linking particulate air pollution and mortality." Research Report Health Effects Institute: 5-114.

Kuik, F., Lauer, A., Churkina, G., Denier van der Gon, H.A.C., Fenner, D., Mar, K.A., and T.M. Butler. 2016. "Air quality modelling in the Berlin-Brandenburg Region using WRF-Chem v3.7.1: sensitivity to resolution of model grid and input data." Geoscientific Model Development 9: 4339-4363.

Kumar, N., Odman, M.T., and A.G. Russell. 1994. “Multiscale air quality modeling: application to Southern California." Journal of Geophysical Research: Atmospheres 99: 5385-5397.

Kumar, N., and A. G. Russel. 1996. "Multiscale air quality modeling of the Northeastern United States." Atmospheric Environment 30(7): 1099-1116.

Laden, F., Schwartz, J., Speizer, F.E., and D.W. Dockery. 2006. "Reduction in fine particulate air pollution and mortality: extended follow-up of the Harvard Six Cities study." American Journal of Respiratory and Critical Care Medicine 173: 667-672.

Lauwaet, D., Viaene, P., Brisson, E., van Noije, T., Strunk, A., Van Looy, S., Maiheu, B., Veldeman, N., Blyth, L., De Ridder, K., and S. Janssen. 2013. “Impact of nesting resolution jump on dynamical downscaling ozone concentrations over Belgium." Atmospheric Environment 67: 46-52. 
Leung, L.R., and Y. Qian. 2003. "The sensitivity of precipitation and snowpack simulations to model resolution via nesting in regions of complex terrain." Journal of Hydrometeorology 4: 1025-1043.

Li, Y., Henze, D.K., Jack, D., and P.L. Kinney. 2015. "The influence of air quality model resolution on health impact assessment for fine particulate matter and its components." Air Qual. Atmos. Health. Online publication.

Liang, J. and M.Z. Jacobson. 2000. "Effects of subgrid segregation on ozone production efficiency in a chemical model." Atmospheric Environment 34(18): 2975-2982.

Mass, C.F., Ovens, D. and K. Westrick. 2002. “Does increasing horizontal resolution produce more skillful forecasts? The results of two years of real-time numerical weather prediction over the Pacific Northwest." Bulletin of the American Meteorological Society 83(3): 407.

Mathur, R., Shankar, U., Hanna, A.F., Odman, M.T., et al. 2005. “Multiscale Air Quality Simulation Platform (MAQSIP): initial applications and performance for tropospheric ozone and particulate matter." Journal of Geophysical Research 110: 1-23.

Matthias, V., Aulinger, A., and M. Quante. 2008. "Adapting CMAQ to investigate air pollution in North Sea coastal regions." Environmental Modelling \& Software 23(3): 356-368.

Menut, L., Coll, I. and S. Cautenet. 2005. "Impact of meteorological data resolution on the forecasted ozone concentrations during the ESCOMPTE IOP2a and IOP2b." Atmospheric Research 74(1-4): 139-159. 
Mensink, C., De Ridder, K., Deutsch, F., Lefebre, F., and K. Van de Vel. 2008. “Examples of scale interactions in local, urban, and regional air quality modelling." Atmospheric Research 89:351-357.

Micea, M., Cappelletti, A., Briganti, G., Vitali, L., Pace, G., Marri, P., Silibello, C., Finardi, S., Calori, G., and G. Zanini. 2010. “Impact of horizontal grid resolution on air quality modeling: a case study over Italy." 13th Conference on Harmonisation within Atmospheric Dispersion Modelling for Regulatory Purposes, pp 166-170.

Miller, K.A., Siscovick, D.F., Sheppard, L., Shepherd, K., Sullivan, J.H., Anderson, G.L., and J.D. Kaufman. 2007. “Long-term exposure to air pollution and incidence of cardiovascular events in women." The New England Journal of Medicine 356: 447-458.

National Center for Atmospheric Research. 2018. "Weather Research and Forecasting Model." Retrieved Aril 26, 2018 from: https://www.mmm.ucar.edu/weather-research-andforecasting-model.

National Research Council (NRC). 2008. “Estimating mortality risk reduction and economic benefits from controlling ozone air pollution." Washington, D.C.: The National Academies Press.

Ontario Regulation (O. Reg.) 419/05: Air Pollution - Local Air Quality. Retreived April 25, 2018 from: https://www.ontario.ca/laws/regulation/050419

Ott, D.K., Kumar, N., and T.M. Peters. 2008. "Passive sampling to capture spatial variability in PM10-25." Atmospheric Environment 42(2): 746-756. 
Palau, J., Pérez-Landa, G., Diéguez, J., Monter, C. and M. Millán. 2005. "The importance of meteorological scales to forecast air pollution scenarios on coastal complex terrain." Atmospheric Chemistry and Physics 5(10): 2771-2785.

Pan, S., Choi, Y., Roy, A., and W. Jeon. 2017. "Allocating emissions to 4 km and 1 km horizontal spatial resolutions and its impact on simulated NOx and $\mathrm{O} 3$ in Houstin, TX." Atmospheric Environment 164: 398-415.

Pappin, A.J., and A. Hakami. 2013a. "Source Attribution of Health Benefits from Air Pollution Abatement in Canada and the United States: An Adjoint Sensitivity Analysis." Environmental Health Perspectives 121: 572-579.

Pappin, A.J., and A. Hakami. 2013b. “Attainment vs. exposure: Ozone metric responses to source-specific NOx controls using adjoint sensitivity analysis." Environmental Science and Technology 47(23): 13519-13527.

Pappin, A.J., Mesbah, S.M., Hakami, A., and S. Schott. 2015. “Diminishing returns or compounding benefits of air pollution control? The case of NOx and ozone." Environmental Science and Technology 49(16): 9548-9556.

Pappin, A. J. 2016a. "Air Quality Modelling for Informing Air Pollution Policy." Carleton University PhD Thesis.

Pappin, A. J., Hakami, A., Blagden, P., Nasari, M., Szyszkowicz, M., and R.T. Burnett. 2016 b. "Health benefits of reducing NOx emissions in the presence of epidemiological and atmospheric nonlinearities." Environmental Research Letters 11(6), 1-10.

Pinault, L., Tjepkema, M., Crouse, D.L., Weichenthal, S., van Donkelaar, A., Martin, R.V., Brauer, M., Chen, H., and R.T. Burnett. 2016. "Risk estimates of mortality attributed to low 
concentrations of ambient fine particulate matter in the Canadian community health survey cohort." Environmental Health 15:18.

Pinault, L., Weichenthal, S., Crouse, D.L., Brauer, M., Erickson, A., van Donkelaar, A, Martin, R.V., Hystad, P., Chen, H., Fines, P., Brook, J.R., Tjepkema, M., and R.T. Burnett. 2017. "Associations between fine particulate matter and mortality in the 2001 Canadian Census Health and Environment Cohort." Environmental Research 159: 406-415.

Pope III, C. A., Burnett, R. T., Thun, M. J., Calle, E. E., Krewski, D., and Thurston, G. D. 2002. “Lung Cancer, Cardiopulmonary Mortality, and Long-term Exposure to Fine Particulate Air Pollution." The Journal of the American Medical Association 287(9): 1132-1141.

Punger, E.M and J.J. West. 2013. "The effect of grid resolution on estimates of the burden of ozone and fine particulate matter on premature mortality in the United States." Air Quality, Atmosphere and Health 6 (3): 563-573.

Queen, A., and Y. Zhang. 2008. "Examining the sensitivity of MM5-CMAQ predictions to explicit microphysics schemes and horizontal grid resolutions, Part III - The impact of horizontal grid resolution." Atmospheric Environment 42(16): 3869-3881.

Ramu, D., Sabeerali, C., Chattopadhyay, R., Rao, D., George, G., Dhakate, A., Salunke, K., Srivastava, A. and S. Rao. 2016. "Indian summer monsoon rainfall simulation and prediction skill in the CFSv2 coupled model: Impact of atmospheric horizontal resolution." Journal of Geophysical Research: Atmospheres 121(5): 2205-2221.

Rauscher, S., Coppola, E., Piani, C. and F. Giorgi. 2009. "Resolution effects on regional climate model simulations of seasonal precipitation over Europe." Climate Dynamics 35(4): 685711. 
Russel, A., and R. Dennis. 2000. "NRSTO critical review of photochemical models and modeling." Atmospheric Environment 34: 2283-2324.

Sandu, A., Daescu, D. N., Carmichael, G. R., and T. Chai. 2005. “Adjoint sensitivity analysis of regional air quality models." Journal of Computational Physics 204(1): 222-252.

Schaap, M., Cuvelier, C., Hendriks, C., Bessagnet, B., Baldasano, J.M., Colette, A., Thunis, P., Karam, D., Fagerli, H., Graff, A., Kranenburg, R., Nyiri, A., Pay, M.T., Rouil, L., Schulz, M., Simpson, D., Stern, R., Terrenoire, E., and P. Wind. 2015. “Performance of European chemistry transport models as function of horizontal resolution." Atmospheric Environment 112: 90-105.

Seinfeld, J.H. and S.N. Pandis. 2006. "Atmospheric chemistry and physics: from air pollution to climate change." 2nd edition, Hoboken, N.J.: Wiley.

Shrestha, K.L., Kondo, A., Kaga, A., and Y. Inoue. 2009. “High-resolution modeling and evaluation of ozone air quality of Okaka using MM5-CMAQ system." Journal of Environmental Sciences 21: 782-789.

Sillman, S., Logan, J.A., and S.C. Wofsy. 1990. "A regional scale model for ozone in the United States with subgrid representation of urban and power plants plumes." Journal of Geophysical Research 95: 5731-5748.

Skamarock, W., Klemp, J., Dudhia, J., Gill, D., Barker, D., Duda, M., Huang, X., Wang, W., and J. Powers. 2008. "A Description of the Advanced Research WRF version 3." Technical Report, National Center for Atmospheric Research, Boulder, CO.

Stohl, A., Wotawa, G., Seibert, P. and H. Kromp-Kolb. 1995. "Interpolation Errors in Wind Fields as a Function of Spatial and Temporal Resolution and Their Impact on Different Types of 
Kinematic Trajectories." Journal of Applied Meteorology 34(10): 2149-2165.

Stroud, C.A., Makar, P.A., Moran, M.D., Gong, W., Gong, S., Zhang, J., Hayden, K., Mihele, C., Brook, J.R., Abbarr, J.P.D., and J.G. Slowik. 2011. "Impact of model grid spacing on regional- and urban- scale air quality predictions of organic aerosol." Atmospheric Chemistry and Physics 11(7): 3107-3118.

Tan, J., Zhang, Y., Ma, W., Yu, Q., Wang, J., and L. Chen. 2015. “Impact of spatial resolution on air quality simulation: A case study in highly industrial area in Shanghai. China." Atmospheric Pollution Research 6(2): 322-333.

Tang, Y. 2002. "A case study of nesting simulation for the Southern Oxidants Study 1999 at Nashville." Atmospheric Environment 36: 1691-1705.

Tesche, T.W., Morris, R., Tonnesen, G., McNally, D., Boylan, J., and P. Brewer. 2006.

“CMAQ/CAMx annual 2002 performance evaluation over the eastern US." Atmospheric Environment 40: 4906-4919.

Thompson, T.M., and N.E. Selin. 2012. "Influence of air quality model resolution on uncertainty assocaited with health impacts." Atmospheric Chemistry and Physics 12: 9753-9762.

Thompson, T. M., Saari, R.K., and N. E. Selin. 2014. “Air quality resolution for health impacts assessment: influence of regional characteristics." Atmospheric Chemistry and Physics 14: 969-978.

Tie, X., Brasseur, G., and Z. Ying. 2010. “Impact of model resolution on chemical ozone formation in Mexico City: application of the WRF-Chem model." Atmospheric Chemistry and Physics 10: 8983-8995 
Turner, M.C., Jerrett, M., Pope-III, C.A., Krewski, D., Gapstur, S.M., Diver, W.R., et al. 2016. "Long-term ozone exposure and mortality in a large prospective study." American Journal of Respiratory and Critical Care Medicine 193(10):1134-42.

U.S. Environmental Protection Agency (U.S. EPA). 2004. “AERMOD: Description of model formulation" Office of Air Quality Planning and Standards, Report R-03-004. Retrieved April 25, 2018 from:

https://www3.epa.gov/scram001/7thconf/aermod/aermod_mfd.pdf

U.S. Environmental Protection Agency (U.S. EPA). 2007. "Guidance on the use of models and other analysis for demonstrating attainment of air quality goals for ozone, $\mathrm{PM}_{2.5}$, and regional haze." Office of Air Quality Planning and Standards, Report B-07-002. Retrieved January 10, 2018 from: https://www3.epa.gov/scram001/guidance/guide/final-03-pmrh-guidance.pdf/

U.S. Environmental Protection Agency (U.S. EPA). 2009. “Integrated Science Assessment (ISA) for Particulate Matter (Final Report)." EPA/600/R-08/139F. Retrieved November 21, 2018 from: https://cfpub.epa.gov/ncea/isa/recordisplay.cfm?deid=216546

U.S. Environmental Protection Agency (U.S. EPA). 2016. “Integrated Science Assessment (ISA) for Oxides of Nitrogen - Health Criteria (Final Report)." EPA/600/R-15/068. Retrieved November 21, 2018 from:

https://cfpub.epa.gov/ncea/isa/recordisplay.cfm?deid=310879

U.S. Environmental Protection Agency (U.S. EPA). 2013. “Integrated Science Assessment (ISA) for Ozone and Related Photo-chemical Oxidants." EPA/600/R-10/076F. Retrieved 
November 21, 2018 from:

https://cfpub.epa.gov/ncea/isa/recordisplay.cfm?deid=247492

U.S. Environmental Protection Agency (U.S. EPA). 2016. "NAAQS Table." Retrieved March 10, 2018 from: https://www.epa.gov/criteria-air-pollutants/naaqs-table

Valarie, M. and L. Menut. 2008. "Does an increase in air quality models' resolution bring surface ozone concentrations closer to reality?" Journal of Atmospheric and Oceanic Technology 25: 1955-1968.

Valin, L.C., Russell, A.R., Hudman, R.C., and R.C. Cohen. 2011. “Effects of model resolution on the interpretation of satellite NO2 observations." Atmospheric Chemistry and Physics 11: 11647-11655.

Vautard, R., Schaap, M., Bergström, R., Bessagnet, B., Brandt, J., Builtjes, P., Christensen, J., Cuvelier, C., Foltescu, V., Graf, A., Kerschbaumer, A., Krol, M., Roberts, P., Rouil, L., Stern, R., Tarrason, L., Thunis, P., Vignati, E., and P. Wind. 2009. "Skill and uncertainty of a regional air quality model ensemble." Atmospheric Environment 43(31), 4822-4832.

Washington State University. 2018. "Community Multiscale Air Quality (CMAQ) Model." Department of Civil and Environmental Engineering. Retrieved April 25, 2018 from: http://bioearth.wsu.edu/cmaq_model.html

Weisman, M.L., Skamarock, W.C., and J.B. Klem. 1997. "The resolution dependence of explicitly modeled convective systems." Monthly Weather Review 125: 527-548.

Wild, O., and M.J. Prather. 2006 "Global tropospheric ozone modeling: quantifying errors due to grid resolution." Journal of Geophysical Research 111:D11305. 
Wolke, R., Schröder, W., Schrödner, R., and R. Eberhard. 2012. "Influence of grid resolution and meteorological forcing on simulated European air quality: A sensitivity study with the modeling system COSMO-MUSCAT." Atmospheric Environment 53: 110-130.

World Health Organization (WHO). 2005. “Air Quality Guidelines - Global Update 2005.

Particulate matter, ozone, nitrogen dioxide and sulphur dioxide.". Retrieved March 15, 2018 from:

http://www.euro.who.int/_data/assets/pdf_file/0005/78638/E90038.pdf?ua=1.

World Health Organization (WHO). 2014. "7 million premature deaths annually linked to air pollution." Retrieved March 15, 2018 from:

http://www.who.int/mediacentre/news/releases/2014/air-pollution/en/

World Health Organization (WHO). 2018. "Ambient (outdoor) air quality and health." Retrieved January 13, 2019 from: https://www.who.int/en/news-room/factsheets/detail/ambient-(outdoor)-air-quality-and-health

Wu, S-Y., Krishnan, S., Zhang, Y., and V. Aneja. 2008a. "Modelling atmospheric transport and fate of ammonia in North Carolina - Part I: Evaluation of meteorological and chemical predictions." Atmospheric Environment 42(14): 3419-3436.

Wu, S-Y., Hu, J-L., Zhang, Y., and V.P. Anega. 2008b. “Modelling atmospheric transport and fate of ammonia in North Carolina - Part II: Effect of ammonia emissions on fine particulate matter formation." Atmospheric Environment 42(14): 3437-3451. 
Yang, Y-J., Wilkinson, J.G. and A.G. Russel. 1997. "Fast, direct sensitivity analysis of multidimensional photochemical models." Environment, Science and Technology 31: 2869-2868.

Yarwood, G., Morris, R.E., and G.M. Wilson. "Particulate Matter Source Apportionment Technology (PSAT) in the CAMx Photochemical Grid Model," in Air Pollution Modeling and its Application XVIIth ed. New York, Springer, 2007, ch. 52: 479-480.

Zeger, S., Dominici, F., McDermott, A., and J. Samet. 2008. "Mortality in the Medicare population and chronic exposure to fine particulate air pollution in urban centers (20002005)." Environmental Health Perspectives 116: 1614-1619.

Zhang, Y., Liu, P., Pun, B., and C. Seigneur. 2006a. "A comprehensive performance evaluation of MM5-CMAQ for the summer 1999 southern oxidants study episode - Part I: Evaluation protocols, databases and meteorological predictions." Atmospheric Environment 40(26): $4825-4838$

Zhang, Y., Liu, P., Pun, B., and C. Seigneur. 2006b. "A comprehensive performance evaluation of MM5-CMAQ for the summer 1999 southern oxidants study episode - Part II: Gas and aerosol predictions." Atmospheric Environment 40(26): 4839-4855. 


\section{Appendix A: Ground-level Ozone Formation Chemistry}

Ozone is a secondary pollutant that is formed in the troposphere through a series of non-linear photochemical chain reactions when precursors (nitrogen oxides, NOx, carbon monoxide, $\mathrm{CO}$, and volatile organic compounds, VOCs) are in the presence of ultraviolet light (from sunlight). The chemistry related to this process is briefly discussed within this Appendix.

The photochemical $\mathrm{NOx}$ cycle occurs when $\mathrm{NO}$ and $\mathrm{NO}_{2}$ are present with sunlight, described by the following reactions (A-1 to A-3) (Seinfeld \& Pandis, 2006):

$$
\mathrm{NO}_{2}+h v \rightarrow \mathrm{NO}+\mathrm{O}
$$

$$
O+O_{2} \rightarrow O_{3}+M
$$

$$
\mathrm{NO}+\mathrm{O}_{3} \rightarrow \mathrm{NO}_{2}+\mathrm{O}_{2}
$$

Reactions $\mathrm{A}-2$ and $\mathrm{A}-3$ are relatively fast compared to $\mathrm{A}-1$, resulting in reaction $\mathrm{A}-1$ as the rate limiting reaction. When sunlight is not present, such as after sunset, $A-3$ depletes $\mathrm{O}_{3}$ rapidly. $\mathrm{O}_{3}$ formation is also governed by the $\mathrm{CO}$ oxidation reactions, described by the following reactions (A-4 to $A-6)$ :

$$
\mathrm{CO}+\mathrm{OH} \rightarrow \mathrm{CO}_{2}+\mathrm{H}
$$

$$
\mathrm{H}+\mathrm{O}_{2}+\mathrm{M} \rightarrow \mathrm{HO}_{2}+\mathrm{M}
$$

$$
\mathrm{HO}_{2}+\mathrm{NO} \rightarrow \mathrm{NO}_{2}+\mathrm{OH}
$$

The $\mathrm{CO}$ oxidation forms the $\mathrm{HO}_{2}$ radical (A-4 and A-5), which in the presence of atmospheric NO, will react to create $\mathrm{NO}_{2}(\mathrm{~A}-6)$. This $\mathrm{NO}_{2}$ formed then takes part in the previously described NOx cycle (A-1 to A-3). The consumption of an $\mathrm{O}_{3}$ molecule by reaction $\mathrm{A}-3$ would occur if $\mathrm{NO}$ 
was not consumed in reaction $\mathrm{A}-6$, and hence, this conversion of $\mathrm{NO}$ to $\mathrm{NO}_{2}$ by $\mathrm{HO}_{2}(\mathrm{~A}-6)$ indirectly produces an $\mathrm{O}_{3}$ molecule. The production of $\mathrm{NO}_{2}$ without the destruction of $\mathrm{O}_{3}(\mathrm{~A}-6)$ is the main source of tropospheric $\mathrm{O}_{3}$ during the day. Depletion of $\mathrm{OH}$ and $\mathrm{NO}_{2}$ by the creation of nitric acid ( $\mathrm{HNO} 3$ ) is one way in which the $\mathrm{O}_{3}$ formation cycle terminates, described by the following reaction (A-7) (Seinfeld and Pandis, 2006):

$$
\mathrm{OH}+\mathrm{NO}_{2}+\mathrm{M} \rightarrow \mathrm{HNO}_{3}+\mathrm{M}
$$

The oxidation of methane $\left(\mathrm{CH}_{4}\right)$ and volatile organic compounds (VOCs) by OH further leads to the formation of $\mathrm{O}_{3}$ in the troposphere. This is shown in the following equations $\mathrm{A}-8$ to $\mathrm{A}-11$, where $\mathrm{RH}$ represents a hydrocarbon of varying chain length.

$$
\begin{gathered}
R H+O H \rightarrow R+\mathrm{H}_{2} \mathrm{O} \\
R+\mathrm{O}_{2}+\mathrm{M} \rightarrow \mathrm{RO}_{2}+M \\
\mathrm{RO}_{2}+\mathrm{NO} \rightarrow \mathrm{RO}+\mathrm{NO}_{2} \\
\mathrm{RO}+\mathrm{O}_{2} \rightarrow \mathrm{R}^{\prime} \mathrm{CHO}+\mathrm{HO}_{2}
\end{gathered}
$$

The $\mathrm{HO}_{2}$ from reaction A-11 reproduces the $\mathrm{OH}$ radical through reaction A-6, creating a cyclic process. The above oxidation reactions terminate through reaction A-7, or through the following reactions A-12 to A-13 (Seinfeld and Pandis, 2006):

$$
\mathrm{HO}_{2}+\mathrm{HO}_{2} \rightarrow \mathrm{H}_{2} \mathrm{O}_{2}+\mathrm{O}_{2}
$$$$
\mathrm{RO}_{2}+\mathrm{HO}_{2} \rightarrow \mathrm{ROOH}+\mathrm{O}_{2}
$$ 
The required radicals $\left(\mathrm{OH}, \mathrm{HO}_{2}, \mathrm{RO}_{2}\right)$ for $\mathrm{O}_{3}$ production are in competition for between $\mathrm{NOx}$ and VOC.

The potential for $\mathrm{NOx}$ to form $\mathrm{O}_{3}$ is dependent on the corresponding spatiotemporal regime made up by the concentration of VOCs and the level of sunlight. The reduction of NOx may cause an increase or decrease in $\mathrm{O}_{3}$, depending on these ambient conditions. The $\mathrm{O}_{3}$ isopleth (Figure $\mathrm{A}-1$ ) describes the non-linear response of $\mathrm{O}_{3}$ to changes in NOx and VOCs concentrations. The non-linearity of the above presented $\mathrm{O}_{3}$ formation equations (A-1 to $\left.A-13\right)$ are displayed by the $\mathrm{O}_{3}$ isopleth in a graphical form.

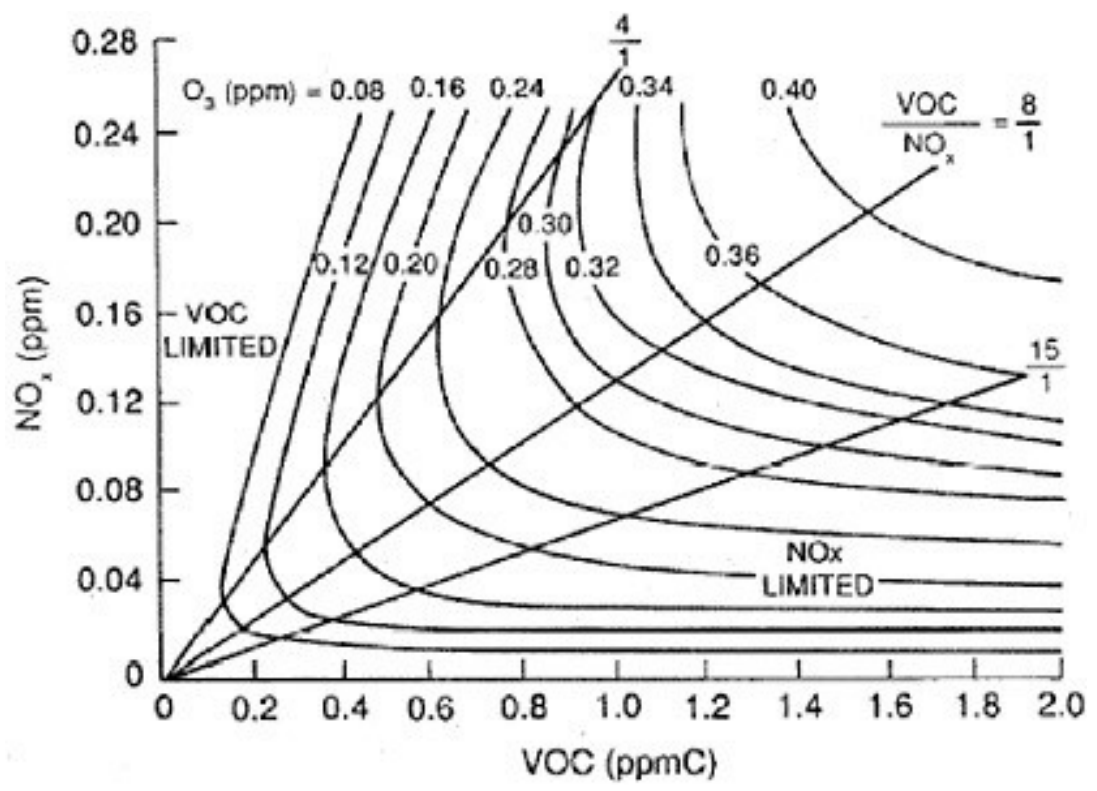

Figure A1. Ozone isopleth describing the ozone formation relationships between NOx and VOC Concentrations (NRC, 2008)

The isopleth contour lines represent the concentration of $\mathrm{O}_{3}$. The location on the isopleth is described by the VOC/NOx ratio. The VOC/NOx ratio is important in understanding the 
response of $\mathrm{O}_{3}$ to changes in $\mathrm{VOC} / \mathrm{NOx}$ emissions. There are three main regions, the NOx inhibited region (VOC limited), the NOx limited region (VOC inhibited) and the $\mathrm{O}_{3}$ ridge. The $\mathrm{O}_{3}$ ridge is the area diagonal from the bottom left corner to the top right in which the production of $\mathrm{O}_{3}$ is maximized. It is described by a VOC/NOx ratio between 4 and 15, depending on the regime. These conditions are typical of sub-urban areas, downwind of NOx emission sources.

Beneath the ridge (right of it) is known as the NOx-limited zone and occurs in low-NOx environments. The production of $\mathrm{O}_{3}$ is directly dependent on reactions $A-6$ and $A-10$, resulting in $\mathrm{O}_{3}$ formation to be directly proportional to the NOx concentration; increases in NOx will increase $\mathrm{O}_{3}$ or conversely, decreases in NOx will decrease $\mathrm{O}_{3}$. There is a positive sensitivity between ozone and NOx in this region. Changes in VOC concentrations will have no effect on $\mathrm{O}_{3}$. This atmospheric regime is seen within rural areas where biogenic $\mathrm{VOC}$ concentrations are high and there are low NOx sources, resulting in low NOx concentrations.

Above the ridge (to the right of it) is known as the NOx inhibited, or VOC limited, zone and occurs in high-NOx environments. Reactions A-6 and A-10 occur as NO is abundant, however, $\mathrm{O}_{3}$ production relies on the availability of radicals that initiate reactions $\mathrm{A}-4$ and $\mathrm{A}-8$. Increases in NOx in these high-NOx regions increases the removal of radicals (e.g. Reaction A-7), terminating the chain reaction and reducing $\mathrm{O}_{3}$. There is a positive sensitivity between VOC and $\mathrm{O}_{3}$ in this region, while there is a negative sensitivity between $\mathrm{NOx}$ and $\mathrm{O}_{3}$. 